\title{
(2) OPEN ACCESS \\ British Society of Gastroenterology consensus guidelines on the management of inflammatory bowel disease in adults
}

\author{
Christopher Andrew Lamb (D) , 1,2 Nicholas A Kennedy (D) ,'4 Tim Raine (D) , \\ Philip Anthony Hendy (D) , ${ }^{6,7}$ Philip J Smith (D) ${ }^{8}$ Jimmy K Limdi (D) , 9,10 \\ Bu'Hussain Hayee (D) , ${ }^{11,12}$ Miranda C E Lomer (D) , ${ }^{12,13}$ Gareth C Parkes (D) , ${ }^{14,15}$ \\ Christian Selinger (D) ${ }^{16,17}$ Kevin J Barrett (D) , ${ }^{18} \mathrm{R}$ Justin Davies (D) , 5,19 \\ Cathy Bennett (D) 20,21 Stuart Gittens (D) ,22 'Malcolm G Dunlop (D) '23,24 \\ Omar Faiz (D) , ${ }^{7,25}$ Aileen Fraser (D) ${ }^{26}$ Vikki Garrick (D), ${ }^{27}$ Paul D Johnston (D) , ${ }^{28}$ \\ Miles Parkes (D) , Jeremy Sanderson (D) , ${ }^{5}{ }^{2}, 13$ Helen Terry (D) ${ }^{28}$ IBD guidelines eDelphi \\ consensus group, Daniel R Gaya (D) ' ${ }^{29,30}$ Tariq H Iqbal (D) , 31,32 Stuart A Taylor (D) , 33,34 \\ Melissa Smith (D), ${ }^{35,36}$ Matthew Brookes (D) , 37,38 Richard Hansen (D) , 27,30 \\ A Barney Hawthorne (D) ${ }^{39}$
}

Additional material is published online only. To view please visit the journal online (http://dx.doi.org/10.1136/ gutjnl-2019-318484).

For numbered affiliations see end of article.

\section{Correspondence to} Dr Christopher Andrew Lamb, Institute of Cellular Medicine, Newcastle University, Framlington Place, Newcastle upon Tyne NE2 4HH, UK; christopher.lamb@newcastle. ac.uk and Dr A Barney Hawthorne, Department of Gastroenterology, University Hospital of Wales, Cardiff, CF14 4XW

barney.hawthorne@wales. nhs.uk

Received 10 February 2019 Revised 10 June 2019 Accepted 10 June 2019 Published Online First 27 September 2019
Check for updates

(c) Author(s) (or their employer(s)) 2019. Re-use permitted under CC BY-NC. No commercial re-use. See rights and permissions. Published by BMJ.

To cite: Lamb CA, Kennedy NA, Raine T, et al. Gut

2019:68:s1-s106

\section{ABSTRACT}

Ulcerative colitis and Crohn's disease are the principal forms of inflammatory bowel disease. Both represent chronic inflammation of the gastrointestinal tract, which displays heterogeneity in inflammatory and symptomatic burden between patients and within individuals over time. Optimal management relies on understanding and tailoring evidence-based interventions by clinicians in partnership with patients. This guideline for management of inflammatory bowel disease in adults over 16 years of age was developed by Stakeholders representing UK physicians (British Society of Gastroenterology), surgeons (Association of Coloproctology of Great Britain and Ireland), specialist nurses (Royal College of Nursing), paediatricians (British Society of Paediatric Gastroenterology, Hepatology and Nutrition), dietitians (British Dietetic Association), radiologists (British Society of Gastrointestinal and Abdominal Radiology), general practitioners (Primary Care Society for Gastroenterology) and patients (Crohn's and
\end{abstract}

Colitis UK). A systematic review of 88247 publications and a Delphi consensus process involving 81 multidisciplinary clinicians and patients was undertaken to develop 168 evidence- and expert opinion-based recommendations for pharmacological, non-pharmacological and surgical interventions, as well as optimal service delivery in the management of both ulcerative colitis and Crohn's disease. Comprehensive up-to-date guidance is provided regarding indications for, initiation and monitoring of immunosuppressive therapies, nutrition interventions, pre-, peri- and postoperative management, as well as structure and function of the multidisciplinary team and integration between primary and secondary care. Twenty research priorities to inform future clinical management are presented, alongside objective measurement of priority importance, determined by 2379 electronic survey responses from individuals living with ulcerative colitis and Crohn's disease, including patients, their families and friends.

\section{CONTENTS}

1 Introduction $\quad$ s6

2 Methodology $\quad$ s6

3 Ulcerative colitis $\quad$ s8

3.1 Diagnosis s8

3.1.1 Histology s9

3.1.2 UC versus Crohn's disease s9

3.2 Phenotypic classification s9

3.3 Clinical and endoscopic disease activity s9

3.4 Treatment targets s9

3.5 Initial treatment of active UC with 5-ASA s10

3.5.1 5-ASA dose $\quad$ s10

3.5.2 Oral and enema 5-ASA s11

3.5.3 Nephrotoxicity s11 
3.6 Corticosteroids in mild to moderate UC

3.6.1 Budesonide MMX

3.6.2 Beclomethasone dipropionate

s12

3.7 Corticosteroids in moderate to severe UC

s12

3.7.1 Failure of oral corticosteroids

s13

3.8 5-ASA maintenance therapy for UC

s13

3.9 Treatment options for UC patients after 5-ASA failure $\quad$ s13

3.9.1 Thiopurines

s13

3.9.2 Infliximab

s13

3.9.3 Adalimumab

s14

3.9.4 Golimumab

s14

3.9.5 Vedolizumab

s14

3.9.6 Tofacitinib

$\mathrm{s} 15$

3.9.7 Ustekinumab

3.9.8 Methotrexate

3.9.9 Choice of immunosuppressive or biological therapy

s16

3.9.10 Therapeutic choice after anti-TNF failure

3.9.10.1 Vedolizumab after anti-TNF therapy

3.9.10.2 Tofacitinib after anti-TNF therapy

3.10 Proctitis

3.10.1 5-ASA suppositories in proctitis

3.10.2 Maintenance therapy for proctitis

$\begin{array}{ll}3.10 .3 \text { Corticosteroid suppositories in proctitis } & \text { s17 }\end{array}$

3.10.4 Treatment options in refractory proctitis $\quad$ s17

3.11 Stopping 5-ASA or thiopurine therapy

3.12 Acute severe ulcerative colitis

3.12.1 Corticosteroid therapy for ASUC

3.12.2 Predictors of outcomes of ASUC

3.12.3 Ciclosporin or infliximab rescue therapy for ASUC s20

$\begin{array}{lr}3.12 .4 \text { Ciclosporin in ASUC } & \text { s20 }\end{array}$

3.12.5 Infliximab in ASUC

3.12.6 Comparison of infliximab and ciclosporin in ASUC

3.12.7 Sequential therapy (infliximab and ciclosporin) in refractory ASUC s22

3.12.8 Accelerated infliximab induction regimen in ASUC s22

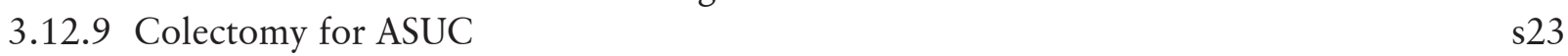

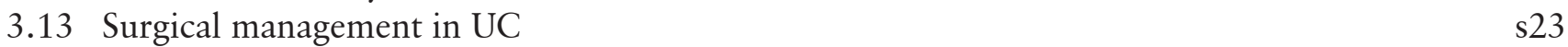

3.13.1 Emergency colectomy for UC $\quad$ s23

$\begin{array}{ll}\text { 3.13.2 Outcomes after colectomy for UC } & \text { s23 }\end{array}$

3.13.3 Surgery in UC patients with primary sclerosing cholangitis $\quad$ s24

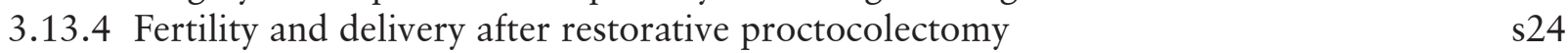

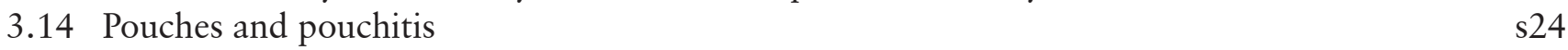

3.14.1 Assessment of new symptoms after IPAA $\quad$ s24

3.14.2 Treatment of acute pouchitis $\quad$ s25

3.14.3 Treatment of chronic pouchitis $\quad$ s25

3.14.4 Treatment with biologics in chronic refractory pouchitis $\quad$ s26

$\begin{array}{lll}3.14 .5 & \text { Follow-up of pouch patients } & \text { s26 }\end{array}$

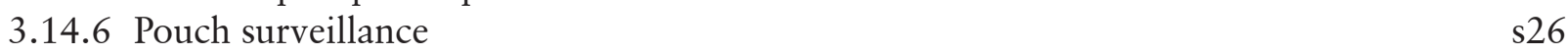

4 Crohn's disease

4.1 Diagnosis, classification and assessment $\quad$ s26

$\begin{array}{lll}\text { 4.1.1 Phenotypic classification } & \text { s27 }\end{array}$

$\begin{array}{ll}\text { 4.1.2 Clinical and endoscopic disease activity } & \text { s27 }\end{array}$ 
4.1.3 Diagnostic tests

4.1.3.1 Cross-sectional imaging: CT, MR and small bowel ultrasound

4.1.3.2 Detection of active disease

4.1.3.3 Investigation of strictures

4.1.3.4 Radiation exposure

4.1.3.5 Capsule endoscopy

4.1.3.6 Balloon-assisted enteroscopy

4.2 Induction of remission in mild to moderate disease ileocolonic disease

4.2.1 Budesonide in ileocaecal Crohn's disease

4.2.2 Corticosteroids in colonic Crohn's disease

4.2.3 Nutritional therapy

4.2.3.1 Exclusive Enteral Nutrition (EEN)

4.2.3.2 Elimination diets

4.2.4 Antibiotic therapy

4.2.5 Surgery in localised ileocaecal Crohn's disease

4.3 Initial treatment of moderate to severe ileocolonic Crohn's disease

4.3.1 Corticosteroids

4.3.2 Early use of biological therapy

4.4 Maintenance treatment in ileocolonic Crohn's disease

4.4.1 Immunomodulator therapy

4.4.1.1 Thiopurine therapy

4.4.1.2 Methotrexate

4.4.2 Mesalazine

4.4.3 Biological therapy with anti-TNF drugs, vedolizumab or ustekinumab

4.4.3.1 Infliximab

4.4.3.2 Adalimumab

4.4.3.3 Choice of anti-TNF agent in Crohn's disease

4.4.3.4 Vedolizumab

4.4.3.5 Ustekinumab

4.4.3.6 Choice of biological therapy after anti-TNF failure

4.4.3.7 Corticosteroid use and infection risk while on anti-TNF therapy

4.4.4 Haematopoietic stem cell transplantation

4.4.5 Leucocyte apheresis

4.5 Proximal jejunal or extensive small bowel disease

4.6 Upper gastrointestinal Crohn's disease

4.6.1 Orofacial granulomatosis

4.7 Stricturing disease

4.7.1 Medical therapy for strictures

4.7.2 Surgical therapy for strictures

4.7.3 Strictureplasty

4.7.4 Endoscopic therapy for strictures

4.8 Non-perianal fistulising Crohn's disease and abscesses

4.8.1 Intra-abdominal abscesses

4.8.2 Medical therapy

4.8.3 Enterovaginal and enterovesical fistulae

4.8.4 Enteroenteric fistulae

4.8.5 Enterocutaneous fistulae

4.8.6 Anti-TNF therapy for non-perianal fistulae

4.9.1 Assessment of perianal disease

4.9.3 Anti-TNF therapy post-surgery for complex perianal fistulae 
4.9.3.1 Infliximab for perianal fistulising disease $\quad \mathrm{s} 40$

4.9.3.2 Adalimumab for perianal fistulising disease $\quad$ s40

4.9.4 Combined surgical treatment with anti-TNF therapy s41

4.9.5 Vedolizumab and ustekinumab therapy s41

4.9.6 Surgical treatment of fistulae $\quad$ s41

4.9.7 Allogeneic adipose-derived stem cell therapy for perianal fistulae s41

$\begin{array}{ll}\text { 4.9.8 Defunctioning stoma formation } & \text { s42 }\end{array}$

4.10 Post-surgical management of Crohn's disease $\quad$ s42

4.10.1 Disease recurrence following ileocolonic resection s42

4.10.1.1 Investigation for symptomatic recurrence following ileocolonic resection s42

4.10.1.2 Non-inflammatory causes of diarrhoea after ileocolonic resection $\quad$ s43

4.10.2 Smoking cessation following surgery $\quad$ s43

4.10.3 Reassessment and medical prophylaxis following ileocolonic resection s43

4.10.3.1 Risk factors for disease recurrence after ileocolonic resection s43

4.10.3.2 Evidence of benefit from medical prophylaxis to prevent postoperative recurrence

5 Common disease considerations

5.1 Infectious diseases and IBD: differential and concurrent diagnoses $\quad \mathrm{s} 45$

5.1.1 Tuberculosis

5.1.2 Enteric infections associated with IBD

5.1.3 Clostridium difficile infection associated with IBD

5.1.4 Cytomegalovirus infection in IBD

5.1.4.1 Treatment of CMV in IBD

5.2 Immunosuppressive therapy

5.2.1 Prevention of infection related to IBD and immunosuppressive therapy s46

5.2.1.1 Epstein Barr Virus

5.2.1.2 Infection risk in patients on anti-TNF therapy

5.2.1.3 Vaccination

5.2.2 Drug management: thiopurines

5.2.2.1 Thiopurine methyltransferase and NUDT15

5.2.2.2 Starting dose for thiopurines

5.2.2.3 Renal excretion of thiopurines

5.2.2.4 Cervical neoplasia and thiopurines

5.2.2.5 Drug monitoring for thiopurines

5.2.2.6 Low-dose thiopurines with allopurinol

5.2.2.7 Thiopurine toxicity

5.2.3 Drug use: methotrexate

5.2.3.1 Methotrexate and pregnancy

5.2.4 Drug management: anti-TNF including biosimilars $\quad$ s52

5.2.4.1 Choice of anti-TNF agent

5.2.4.2 Biosimilar anti-TNF drugs

5.2.4.3 Assessment after anti-TNF induction therapy

5.2.4.4 Drug levels of infliximab and adalimumab

5.2.4.5 Primary non-response to anti-TNF therapy

5.2.4.6 Secondary loss of response to anti-TNF therapy

5.2.4.7 Annual review of patients on biologics therapy

5.2.5 Drug management: vedolizumab and ustekinumab

5.2.5.1 Vedolizumab

5.2.5.2 Ustekinumab s55

5.2.5.3 Extraintestinal manifestations (EIM) of IBD and vedolizumab therapy s55 
5.2.7 Withdrawal of immunosuppression for patients in sustained remission s56

5.2.7.1 Stopping thiopurines $\quad$ s56

5.2.7.2 Stopping anti-TNF therapy s56

5.2.7.3 Monitoring following immunosuppressive and biological therapy withdrawal

5.2.8 Drug management: corticosteroids

5.2.8.1 Overuse of corticosteroids

5.2.8.2 Corticosteroid-related bone disease

s56

5.2.8.3 Calcium and vitamin D supplementation

s56

s56

s57

5.2.8.4 Corticosteroid-related adrenal suppression

s57

5.2.8.5 Other metabolic complications of corticosteroids

s58

5.2.8.6 Prevention of Pneumocystis jirovecii infection

s59

s59

5.3 Diet and IBD

s59

s59

5.3.1 Nutritional assessment and monitoring

s60

5.3.2 Blood tests for specific nutritional deficiencies

$\mathrm{s} 60$

5.3.3 Vitamin D

5.3.4 Dietary treatment of functional symptoms in IBD

s61

5.4 Preoperative optimisation

s61

5.4.1 Preoperative nutritional status

5.4.1.1 Evidence for preoperative nutritional support

s62

s62

5.4.2 Preoperative serum albumin

s62

5.4.3 Preoperative anaemia

s62

5.4.4 Preoperative corticosteroids

s62

5.4.5 Preoperative thiopurines $s 63$

$\begin{array}{lll}\text { 5.4.6 Preoperative anti-TNF therapy } & \text { s63 }\end{array}$

5.4.7 Summary of recommendations for operative optimisation $\quad$ s63

5.5 Faecal calprotectin $\quad$ s63

5.5.1 Faecal calprotectin in the differential diagnosis of IBD versus IBS s63

5.5.1.1 Faecal calprotectin in the assessment of disease activity in known IBD s64

5.5.1.2 Use of faecal calprotectin to inform therapy changes s64

5.5.2 Collection of faecal calprotectin samples

5.6.1 Flexible sigmoidoscopy and colonoscopy in acute colitis $\quad$ s64

5.6.2 Bowel preparation for colonoscopy $\quad$ s66

5.7 Drug treatment adherence

s66

$\begin{array}{lll}\text { 5.7.1 Strategies to improve adherence } & \text { s66 }\end{array}$

5.8 Smoking

5.8.1 Smoking and Crohn's disease

5.8.2 Smoking and UC

5.9 Psychology and stress

$\begin{array}{lll}\text { 5.9.1 Treatment of anxiety and depression in IBD } & \text { s67 }\end{array}$

5.10 Pain and fatigue in IBD

5.10.1 Psychological therapy for unexplained pain in IBD

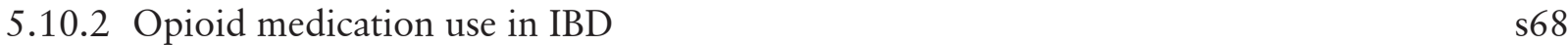

5.10.3 Fatigue in IBD

5.10.3.1 Investigations and treatment in IBD patients with fatigue

5.10.3.2 Non-pharmacological therapy for fatigue in IBD 
5.12.1 Probiotics, prebiotics and synbiotics in IBD $s 69$

$\begin{array}{lll}\text { 5.12.2 } & \text { Faecal microbial transplantation } & \text { s70 }\end{array}$

$\begin{array}{ll}\text { 5.12.3 Complementary and alternative therapy for IBD } & \text { s70 }\end{array}$

$\begin{array}{ll}\text { 5.12.3.1 Cannabis extracts } & \text { s70 }\end{array}$

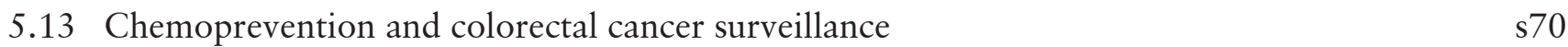

$\begin{array}{ll}\text { 5.13.1 Surveillance ileocolonoscopy } & \text { s70 }\end{array}$

$\begin{array}{ll}\text { 5.13.2 IBD-related cancer chemoprevention with mesalazine } & \text { s71 }\end{array}$

$\begin{array}{ll}\text { 5.13.3 IBD-related cancer chemoprevention with thiopurines } & \text { s71 }\end{array}$

5.14 Anti-TNF, vedolizumab, ustekinumab and tofacitinib therapy during pregnancy s71

$\begin{array}{lll}\text { 5.14.1 Fetal and infant exposure to anti-TNF drugs } & \text { s71 }\end{array}$

5.14.2 Cessation of anti-TNF therapy in the second trimester in quiescent IBD $\quad$ s72

5.14.3 Continuation throughout pregnancy in IBD patients at high risk of flare $\quad$ s73

$\begin{array}{ll}\text { 5.14.4 Risk to the fetus of continuing anti-TNF until delivery } & \text { s73 }\end{array}$

5.14.5 Long-term outcomes of anti-TNF therapy during pregnancy $\quad$ s73

$\begin{array}{ll}\text { 5.14.6 Infant vaccinations after exposure to biologics } & \text { s73 }\end{array}$

5.14.7 Vedolizumab, ustekinumab and tofactinib in pregnancy $\quad$ s74

5.15 Genetics

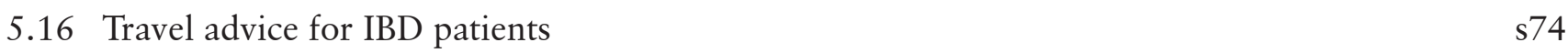

6 Service delivery $\quad s 75$

$\begin{array}{lll}6.1 & \text { IBD service and the multidisciplinary team } & \text { s75 }\end{array}$

$\begin{array}{ll}\text { 6.1.1 MDT meetings } & \text { s75 }\end{array}$

$\begin{array}{ll}\text { 6.2 Quality improvement and audit } & \text { s76 }\end{array}$

$\begin{array}{lll}\text { 6.2.1 Rapid assessment } & \text { s76 }\end{array}$

6.2.2 Adverse event monitoring $\quad$ s76

6.3 Electronic patient data collection for clinical management and audit $\quad$ s76

$\begin{array}{lll}6.4 & \text { IBD specialist nurses } & \text { s76 }\end{array}$

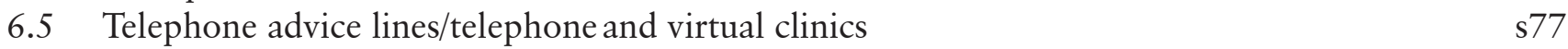

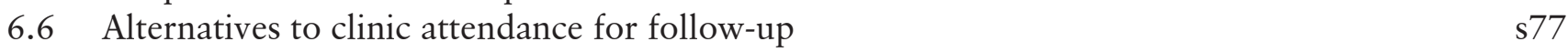

$\begin{array}{llr}6.7 & \text { Self-management } & \text { s78 }\end{array}$

$\begin{array}{llr}6.8 & \text { Primary care } & \text { s78 }\end{array}$

$\begin{array}{llr}7 & \text { Future research priorities } & \text { s79 }\end{array}$

$\begin{array}{llr}8 & \text { Concluding remarks } & \text { s79 }\end{array}$

$\begin{array}{lr}\text { References } & \text { s82 }\end{array}$

\section{INTRODUCTION}

In the past decade there have been major advances in investigations, pharmacological, non-pharmacological and surgical interventions for both ulcerative colitis (UC) and Crohn's disease. In light of this, the British Society of Gastroenterology (BSG) Clinical Services and Standards Committee (CSSC) commissioned a new guideline for the management of inflammatory bowel disease (IBD) in adults. This was to replace the 2011 iteration of IBD guidelines from the Society. ${ }^{1}$ The aim of this document is to provide high-quality disease management guidance for healthcare professionals managing IBD, to ensure that investigation, treatment and monitoring decisions are based on the best available evidence, and to promote and improve best accepted practice. Where appropriate the guidelines refer to relevant National Institute for Health and Care Excellence (NICE) documents, with attention drawn to how this guidance can be incorporated into the UK National Health Service (NHS) infrastructure and funding pathways.

\section{METHODOLOGY}

The guideline is of relevance to adults aged 16 years and over and was developed according to Grading of Recommendations Assessment, Development, and Evaluation (GRADE) methodology, ${ }^{2}$ in accordance with the principles of the AGREE II tool, ${ }^{3}$ and in compliance with the BSG Guidelines Advice Document. ${ }^{4}$ The completed document was formally peer reviewed by the BSG CSSC and BSG Council prior to submission for publication. The guideline writing process was supported by regular consultation from Professor Cathy Bennett (Systematic Research Ltd and Royal College of Surgeons in Ireland (RCSI)) and used a bespoke online platform developed by Dr Stuart Gittens (ECD Solutions: https://www.guideline.pub/bsg-ibd/) to develop clinical questions structured by Population, Intervention, Comparator and Outcome (PICO) or Population, Exposure, Outcome (PEO) development, to assimilate evidence and to facilitate voting of draft statements and recommendations using a modified eDelphi process. 
After commissioning of the guideline by the BSG CSSC, a Guideline Development Group (GDG) was convened by the Chair of the IBD Section Committee of the BSG (ABH). A GDG Lead (CAL) and conflicts of interest Chair (TI) were appointed. Key Stakeholders from the following groups were represented: British Society of Gastroenterology (BSG), Association of Coloproctology of Great Britain and Ireland (ACPGBI), Royal College of Nursing (RCN), British Society of Paediatric Gastroenterology, Hepatology and Nutrition (BSPGHAN), British Dietetic Association (BDA), British Society of Gastrointestinal and Abdominal Radiology (BSGAR), and the Primary Care Society for Gastroenterology (PCSG). Patient representation was provided by Crohn's and Colitis UK.

Members of the BSG IBD Section Committee were invited to take part in the GDG along with external clinicians with relevant experience. The GDG and all conflicts of interest for 12 months preceding GDG formation were vetted and approved by the BSG CSSC.

Clinical priorities to be covered by the guideline were set by the GDG including:

- Definitions, clinical features and diagnosis

- Investigations including imaging

- Treatment of active UC including surgery and acute severe UC (ASUC)

- Pouchitis management

- Treatment of active Crohn's disease (ileal, ileocolonic, colonic, jejunal, upper GI, perianal)

- Maintenance treatment of Crohn's disease

- Surgery for Crohn's disease (including non-perianal fistulising disease)

- Common considerations for drug groups to include mesalazines, corticosteroids, thiopurines, methotrexate, ciclosporin, anti-TNF, vedolizumab, ustekinumab, tofacitinib and antibiotics

- Therapeutic monitoring including drug levels and drug toxicity/immunogenicity, and pre-treatment infection screening and vaccination

- Non-drug therapies including leucocyte apheresis and stem cell transplantation

- Nutrition and dietary therapy

- Lifestyle factors including smoking

- Pain and fatigue

- Psychological aspects

- Service delivery

- Primary care management of IBD

Where substantial up-to-date guidance existed on special circumstances-for example, pregnancy, osteoporosis, iron deficiency, immunosuppression in the context of prior malignancy or histology-extensive systematic review would not be performed but summary data would be presented to encourage best practice with referencing to signpost other guidance. Guidance for surgical technique in IBD would not be extensively covered due to a concurrent guideline development process in this area led by the ACPGBI. ${ }^{5}$ Health economics and costs of drugs would not be assessed as part of the guideline, although cost would be mentioned as an important consideration when there is a choice of treatments.

A clinical framework was then designed to visually map and group patient management decisions and influencing clinical factors, including disease location and severity. Sub-categorisations were made to identify aspects pertinent to pharmacological and non-pharmacological intervention, nutrition, imaging, surgery, primary care and service delivery. Four working groups were formed (led by NAK, TR, PH and PJS alongside CAL and
$\mathrm{ABH})$ to draft and develop a list of key thematic and sub-thematic clinical questions grouped into sections defined by the clinical framework that face IBD clinicians in everyday healthcare practice. These clinical questions were circulated to all stakeholder groups for review by members outside the GDG to ensure all relevant areas of clinical practice were covered. Following stakeholder review, the list was further developed producing 54 thematic questions with 360 associated clinical questions grouped around these themes (see online supplementary appendix 1).

Next, the clinical questions were further revised, refined and combined with the thematic questions in order to design the systematic review. Keyword tables derived from these questions and formulated according to PICO or PEO structure were generated on the online platform, and structured searches of electronic literature databases were performed. The literature searches were designed, run in electronic databases and exported to Endnote reference managing software, supported by information specialists at York Health Economics Consortium. Searches of the Medline and EMBASE databases were performed in March 2017 and updated in March 2018. No date or study design limits were incorporated into searches in order to return all available evidence, including conference proceedings (although conference proceeding returns were limited to 5 years preceding the date of search). The search strategy used is presented in online supplementary appendix 2. In this way, systematic literature searches and reviews were undertaken to identify and synthesise evidence to support the creation of statements with supporting narrative syntheses of evidence. A total of 87959 references were returned after deduplication from these searches. Focused top-up searches using keywords were performed until June 2019 to ensure evidence was up to date at the time of submission for publication. GDG members were able to also propose papers or electronic documents (eg, NICE guidance) for inclusion in the literature databases throughout the guideline development process. In this manner an additional 288 entries were added to the reference library to make a total of 88247 . References were cross-searched both manually using keywords and Boolean operators, and using a bespoke programmatic algorithm (the latter cross-referencing content of abstract, title and keywords with contents of PEO and PICO tables), both facilitated by the online platform. Literature was assessed according to the pre-designed PEO and PICOs, and abstracts \pm full text assessed for relevance and quality. Evidence-based evaluative text and associated reference lists were developed along with draft statements and grouped/archived in a customised electronic database. Statements considered potential health benefits, side effects and risks of recommendations to patients, as well as cost and service implications. Full economic analyses were not undertaken.

Following statement revision by the GDG according to Delphi methodology, an 'IBD guidelines eDelphi consensus group' of 81 clinicians and patients was formed consisting of representatives invited from all stakeholder groups listed above, and all members of the GDG except CB and SG who did not vote. A modified eDelphi mechanism process, employing the online platform, was then used to produce an evidence-based consensus, following a NICE accredited methodology. This consisted of three main rounds of anonymous web-based voting, using a custom-built online voting platform scoring each using a 5 -point scale with updated iterations of the statements and evaluative text based on feedback after each round.

Following two rounds of anonymised voting, statements conforming to PICO/PEO which achieved consensus of $80 \%$ 
agreement or higher were categorised according to the GRADE system for grading quality of evidence and strength of recommendations. Assessments were made independently by two members of the GDG (blinded to one another's assessment) using a custom-built electronic database by NAK in REDCap ${ }^{6}$ (at https://surveys.exeteribd.org.uk/). All assessments were reviewed and where necessary moderated by $\mathrm{CAL}$ and $\mathrm{ABH}$ to determine agreement. To assess the quality of evidence for each statement, each member considered study type, risk of bias, inconsistency, indirectness, imprecision, publication bias, effect size, plausible confounding variables and dose-response gradient if applicable. The quality of evidence ranged from 'high' (further research is very unlikely to change confidence in the estimate of effect), 'moderate' (further research is likely to have an important impact on confidence in the estimate of effect and may change the estimate), 'low' (further research is very likely to have important impact on confidence in the estimate of effect and is likely to change the estimate), and 'very low' (any estimate of effect is very uncertain). The strength of recommendation was assessed based on considerations of desirable and undesirable anticipated effects, the certainty of the evidence of effects, any important uncertainty about or variability in how much people value the outcome, whether the balance of these effects favours the intervention or comparison, the acceptability of intervention to key stakeholders and feasibility of intervention implementation. The strength of each recommendation was then recorded as 'strong' (meaning that benefits clearly outweigh risks and burdens or vice versa) and conditional recommendations as 'weak' (where benefits, risks and burdens are conditional, closely balanced or uncertain).

Where statements did not conform to $\mathrm{PICO} / \mathrm{PEO}$ (such as subjective interventions or where outcomes were multiple) and evidence was indirect or of low quality, recommendations to inform clinical practice were presented as Good Practice Recommendations and listed separately to GRADE recommendations, but still underwent consensus voting.

The GDG voted on all statements and Good Practice Recommendations, and other eDelphi participants voted on one of three subsets of statements and Good Practice Recommendations in order to ensure adequate numbers of responses were obtained for each, that expertise was equally distributed across subject areas and that surgeon members of the group voted on all surgical-related topics. The total number of respondents per statement and recommendation are presented in online supplementary table 3 . Statements and recommendations not reaching $80 \%$ consensus agreement following three rounds of voting were removed and are presented in online supplementary appendix 3 .

\section{Conflict of interest}

To ensure transparency and declaration of any potential bias, all the GDG members were asked to declare a minimum of 12 months competing financial and non-financial interests when they joined the group and during initial statement drafting. All members of the GDG and IBD guidelines eDelphi consensus group also declared conflicts of interest before each of the three rounds of Delphi consensus voting. This is in keeping with the NICE approved processes. The final submitted conflicts of interest for each member is shown in online supplementary table 2. eDelphi participants were asked to abstain from voting where they either did not have sufficient knowledge to vote on a particular statement or where they identified themselves as having a conflict which precluded voting. The number of abstentions in the final round of voting is presented in online supplementary table 3 .

\section{Areas of unmet research priority identified by systematic review}

Following systematic review, the original 414 clinical questions defined by the group, evaluative descriptions for each statement and the results of consensus voting were reviewed to determine where evidence was insufficient to provide recommendations for practice, or where further research was desirable to define and support best clinical practice. From this, 20 areas of research priority with associated questions were determined and described for a healthcare professional readership. General audience versions of these questions were then written and readability assessed by the Flesch Reading Ease and Flesch-Kincaid Grade Level (see online supplementary table 4). The importance to patients, their relatives, partners, parents, friends and carers of these 20 research priority themes was determined by two electronic surveys conducted by the UK patient charity Crohn's and Colitis UK during December 2018 and January 2019 using the general audience versions and asking survey respondents to score each priority on a Likert scale of 1-9 (1=notimportant and $9=$ very important). Survey 1 was promoted by social media and Survey 2 through a combination of social media and direct email to respondents to Survey 1 who agreed to be contacted for future survey.

\section{Revisions of the guideline}

We suggest that every 2 years a formal assessment of the following should take place:

- Research objectives identified by the guidelines should be reviewed for evidence of additional studies, contributing to resolving the objective

- Review of new evidence that may change former recommendations

- Identification of any error in the guidelines after publication

- Exploration of any evidence of inequality in access to services between different social groups that can be addressed through guideline recommendations

- Review of any new technology or drugs or legislation, that will change former recommendations

We suggest the guidelines should be fully updated after 5 years. All materials are archived using the online system at https://www. guideline.pub/bsg-ibd/.

\section{Note on drug nomenclature}

In this document we have adopted the following descriptors: 'Immunosuppressive drugs': corticosteroids, thiopurines, methotrexate, calcineurin inhibitors, janus kinase inhibitors and all biologic drugs where the mode of action is immunosuppressive; 'Immunomodulators': thiopurines and methotrexate; 'biologics': monoclonal antibody drugs.

\section{ULCERATIVE COLITIS}

\subsection{Diagnosis}

Ulcerative colitis (UC) is a chronic inflammatory disease characterised by mucosal inflammation starting distally in the rectum, with continuous extension proximally for a variable distance, often with an abrupt demarcation between inflamed and non-inflamed mucosa. Typically, patients with UC experience periods of relapse and remission. Up to $90 \%$ will have one or more relapses after the first attack, and early relapse or active disease 
Statement 1. Where ulcerative colitis is diagnosed by sigmoidoscopy, we recommend a full ileocolonoscopy to delineate disease extent, severity of inflammation and to exclude Crohn's disease (GRADE: strong recommendation, very low-quality evidence. Agreement: 100\%).

in the first 2 years is associated with a worse disease course subsequently. ${ }^{78}$

In patients presenting with suspected UC, stool cultures and Clostridium difficile toxin assay should always be performed to rule out infective causes. While UC is often initially diagnosed at flexible (or rigid) sigmoidoscopy, it is important to confirm the diagnosis, extent and severity of disease by means of full ileocolonoscopy, usually within the first year, as this can more definitively confirm the diagnosis of UC versus Crohn's disease and give information that may help to predict future disease course, including potential and risk stratification for dysplasia, ${ }^{9}$ and thus will influence treatment choices. For histological assessment at least two biopsy specimens should be taken from five sites throughout the examined bowel, including the ileum and rectum, during the initial endoscopic evaluation.

Rectal sparing in UC has been described in up to $>3 \%$ of patients, ${ }^{10}$ but more frequently patchy inflammation of the rectum may be seen in those who have been given empirical topical therapy. ${ }^{11} 12$ The presence of a 'caecal patch', isolated peri-appendiceal inflammation and backwash ileitis can occur in UC, but if the histology and clinical pattern are not otherwise typical of UC, then small bowel evaluation is required to exclude Crohn's disease (see Section 4.1.3.1: Crohn's disease, Cross-sectional imaging: CT, MR and small bowel ultrasound). Backwash ileitis has been reported in up to $20 \%$ of patients with extensive colitis. $^{13}$

\subsubsection{Histology}

No histological feature is diagnostic of UC, but the combination of basal plasmacytosis, diffuse crypt atrophy and distortion, villous surface irregularity and mucus depletion are suggestive of a diagnosis of UC in the correct clinical context. ${ }^{14}$ Uneven distribution of inflammation within the colon or within biopsies can occur in patients with long-standing disease, or after treatment.

\subsubsection{UC versus Crohn's disease}

In $5-15 \%$ of IBD patients, endoscopic and histological assessments cannot distinguish between Crohn's colitis and UC, and these patients are labelled as IBD-unclassified (IBD-U), or if features are still indeterminate after colectomy histology is assessed, described as indeterminate colitis. ${ }^{15-17}$ IBD-U is more common in children than adults. ${ }^{18}$ In a small proportion of UC patients their diagnosis is later changed to IBD-U or Crohn's disease. $^{19-21}$

\subsection{Phenotypic classification}

The Montreal classification ${ }^{22}$ in adults and Paris classification $^{23}$ in children (table 1) are useful in ascribing phenotypes to patients both for treatment and to assist with service delivery and research. ${ }^{24}$ Children developing IBD generally have more extensive disease than adults. ${ }^{25}$ Establishing the extent of the inflammation in a patient with UC is important for prognosis as the likelihood of colectomy is dependent on disease extent. A systematic review showed that the 10 year colectomy rate is $19 \%$ for those with extensive colitis, $8 \%$ with left-sided colitis and $5 \%$ with proctitis; and male gender, young age and elevated
Table 1 Montreal and Paris classification in UC

\begin{tabular}{|c|c|c|c|c|}
\hline \multirow[b]{2}{*}{ Extent $^{*}$} & \multicolumn{2}{|c|}{ Montreal $^{22}$} & \multicolumn{2}{|c|}{ Paris $^{23}$} \\
\hline & E1 & Ulcerative proctitis & E1 & Ulcerative proctitis \\
\hline & E2 & $\begin{array}{l}\text { Left-sided UC (distal to } \\
\text { splenic flexure) }\end{array}$ & E2 & $\begin{array}{l}\text { Left-sided UC (distal to } \\
\text { splenic flexure) }\end{array}$ \\
\hline & E3 & $\begin{array}{l}\text { Extensive (proximal to } \\
\text { splenic flexure) }\end{array}$ & E3 & $\begin{array}{l}\text { Extensive (hepatic flexure } \\
\text { distally) }\end{array}$ \\
\hline & & & E4 & $\begin{array}{l}\text { Pancolitis (proximal to } \\
\text { hepatic flexure) }\end{array}$ \\
\hline \multirow[t]{4}{*}{ Severity } & SO & Clinical remission & SO & Never severe† \\
\hline & S1 & Mild UC & S1 & Ever severet \\
\hline & S2 & Moderate UC & & \\
\hline & S3 & Severe UC & & \\
\hline
\end{tabular}

*Extent defined as maximal macroscopic inflammation.

†Severe defined by Paediatric Ulcerative Colitis Activity Index (PUCAI) $\geq 65$.

inflammatory markers at diagnosis also increase the likelihood of colectomy. ${ }^{26}$ Backwash ileitis is also associated with more aggressive disease, and with primary sclerosing cholangitis. ${ }^{13}$ Those with extensive colitis also have the highest risk of developing colorectal cancer. ${ }^{27} 28$

Disease extent can change after diagnosis. ${ }^{29} \mathrm{Up}$ to half with proctitis or proctosigmoiditis will develop more extensive disease. ${ }^{82630}$ Of patients with proctitis initially, 10\% will ultimately have extensive colitis. ${ }^{31}$ However, over time the extent of inflammation can also regress, and classification should always remain as the maximal extent. ${ }^{29}$ Endoscopic appearance may significantly underestimate the true extent (particularly in quiescent UC), and this should be confirmed by mapping biopsies.

\subsection{Clinical and endoscopic disease activity}

Definitions in relation to disease activity are shown in box 1. The Mayo Score for UC is widely used in clinical trials and may be applied to clinical practice as a composite clinical and endoscopic tool (table 2). ${ }^{32}$ The score of $0-12$ includes a measure of stool frequency, rectal bleeding, a physician's global assessment and a measure of mucosal inflammation at endoscopy. The partial Mayo score uses the non-invasive components of the full score and correlates well to patient perceptions of response to therapy. ${ }^{33}$

There is wide variation in interpretation of disease activity endoscopically. ${ }^{34}$ The Ulcerative Colitis Endoscopic Index of Severity (UCEIS) has been developed to improve reliability (table 3). ${ }^{35} 36$ The Modified Mayo Endoscopic Score is another simple measure of endoscopic activity that correlates well with clinical and biological activity (table 4). ${ }^{37}$ Although both have been extensively validated, inter-observer variation remains a significant limitation for these visual scores. ${ }^{38} 39$

Symptomatic and endoscopic scores may be limited by their ability to quantify accurately the impact of disease on quality of life, including fatigue and psychosocial function, or if complex the indices may be difficult to apply to clinical practice. ${ }^{4041}$ An increasing emphasis on patient reported outcomes measures (PROMs: standardised questionnaires filled out by patients without clinician involvement) in clinical trials may translate to routine clinical practice (box 1$).^{42}$

\subsection{Treatment targets}

The ultimate target of medical therapy is a contentious issue as there is no fully agreed or validated definition of remission, although many parameters have been suggested both clinically and endoscopically. ${ }^{43-45}$ Using mucosal healing as a treatment 


\section{Box 1 Definitions in IBD management}

- Steroid dependency: inability to wean systemic steroids below $10 \mathrm{mg}$ prednisolone within 3 months without recurrent active disease, or symptomatic relapse of IBD within 3 months of stopping steroids. ${ }^{1279}$

- Steroid refractory: inability to induce symptomatic remission with systemic steroids. ${ }^{1279}$

- Mucosal healing: typically, this refers to absence of macroscopic mucosal inflammation or ulceration, although precise definitions between studies vary. While usually defined endoscopically, more recently there has also been interest in 'histological healing'. ${ }^{56}$

- Treat to target: the goal being more than symptomatic remission with the intention of reducing risk of future relapse or complications (eg, ileal strictures or colon cancer) resulting from uncontrolled but asymptomatic inflammation. ${ }^{56360}$ Future studies are required to define targets, the practicality of achieving targets and to examine the balance of benefit versus risk and cost.

- Therapeutic drug monitoring (TDM): measurement of drug ( \pm anti-drug antibody) levels to assess compliance, drug metabolism and immunogenicity with a view to guide dose adjustments or switch off therapy (eg, where patients are relapsing despite therapeutic levels, or have developed high titre anti-drug antibodies with low levels).

- Patient related outcome (PRO): a report directly from patients about how they feel or function in relation to their IBD and its therapy without interpretation by healthcare professionals. Provides an indication of the outcome of an intervention, or quality of care. ${ }^{1280}$

- Patient reported outcome measure (PROM): a tool or instrument used to measure PROs. ${ }^{1280}$

target is contentious because of the implications for clinical practice, with the need for more endoscopic assessment and likely escalation of therapy in asymptomatic patients. ${ }^{46}$ In an Australian retrospective study, $61 \%$ of 246 patients were in clinical remission, but only $35 \%$ were in both clinical and endoscopic remission (Mayo endoscopic subscore $\leq 1$ ), and only $16 \%$ of the 246 patients were also in histological remission. ${ }^{47}$ Using the Mayo endoscopic subscore, there is no consensus on the value of achieving a score of 0 rather than 1 , with documented differences in future disease course between the two. ${ }^{46}$ There is lack of clear evidence about the importance of histological remission as well as endoscopic remission. ${ }^{48}{ }^{49}$ Histological remission has now become an end-point for some new drug trials in UC, but there remains lack of agreement about the definition, and the evidence of long-term benefit is only from observational studies. ${ }^{44} 48$ 50-54 There is, however, growing evidence that the presence of endoscopic and histological inflammation is predictive of future flares, lack of sustained remission, need for corticosteroids and colectomy. ${ }^{44} 48$ 50-54 There is likewise evidence of the benefits of mucosal healing in reducing future risk of colorectal carcinoma $^{55}$ (see Section 5.13: Chemoprevention and colorectal cancer surveillance). There is a growing consensus therefore that the target for UC should be clinical and/or patient-reported remission (defined as absence of rectal bleeding and return to normal bowel habit) combined with endoscopic remission (Mayo endoscopic subscore of $\leq 1$ ) ${ }^{56}$ More evidence is needed of the implications for a more aggressive approach to achieving mucosal healing, including the acceptability to patients of increased medication with potential toxicity (often despite lack of clinical symptoms). A widely agreed definition is needed for both endoscopic healing and histological healing that can be used in clinical practice, and more evidence is also needed of the role of faecal calprotectin or other biomarkers as non-invasive surrogates for mucosal healing. Longer-term prospective studies of mucosal healing will be needed to provide evidence of the risks and benefits of this approach.

\subsection{Initial treatment of active UC with 5-ASA}

Oral 5-ASA (5-aminosalicylic acid) is the standard therapy for mild to moderately active UC. Meta-analyses support the efficacy of oral 5-ASA for induction therapy for mild to moderately active UC. ${ }^{5758}$ Once daily dosing is as effective as divided doses. $^{59}$ Doses $\geq 2 \mathrm{~g} /$ day are more effective than dosages $<2 \mathrm{~g} /$ day for remission (RR $0.91 ; 95 \% \mathrm{CI} 0.85$ to 0.98 ). ${ }^{60}$ The majority of patients with mild to moderate UC will respond to 2-3 g 5-ASA (depending on formulation used) and higher doses can be used in those with more severe symptoms or those not responding initially.

\subsubsection{5-ASA dose}

The ASCEND trials investigated the dose-response effect of 5-ASA (Asacol) for the induction of response in UC. ASCEND I randomised 301 patients with mild-moderately active UC to $2.4 \mathrm{~g}$ or $4.8 \mathrm{~g}$ of mesalazine ${ }^{61}$ At week 6 , a similar proportion of patients experienced improvement in either group $(51 \%$ vs $56 \%$, $\mathrm{p}=\mathrm{NS}$ ). When results were stratified according to disease severity, patients with moderate disease had better response to $4.8 \mathrm{~g} /$ day, but there was no significant difference in dose-response in those with mildly active disease. ASCEND II confirmed that patients

Table 2 Mayo score for ulcerative colitis ${ }^{32}$

\begin{tabular}{|c|c|c|c|c|}
\hline Mayo index & 0 & 1 & 2 & 3 \\
\hline Stool frequency & Normal & $1-2 /$ day more than normal & $3-4 /$ day more than normal & $5 /$ day more than normal \\
\hline Rectal bleeding & None & $\begin{array}{l}\text { Streaks of blood with stool }<50 \% \text { of } \\
\text { the time }\end{array}$ & Obvious blood with stool most of time & Blood passed without stool \\
\hline Mucosa (endoscopic subscore) & $\begin{array}{l}\text { Normal or inactive } \\
\text { disease }\end{array}$ & $\begin{array}{l}\text { Mild disease (erythema, decreased } \\
\text { vascular pattern, mild friability) }\end{array}$ & $\begin{array}{l}\text { Moderate disease (marked erythema, lack of } \\
\text { vascular pattern, friability, erosions) }\end{array}$ & $\begin{array}{l}\text { Severe disease (spontaneous } \\
\text { bleeding, ulceration) }\end{array}$ \\
\hline Physician's global assessment & Normal & Mild disease & Moderate disease & Severe disease \\
\hline
\end{tabular}

Mayo score=sum of scores for each of the four variables (maximum score 12).

Clinical response: reduction of baseline Mayo score by $\geq 3$ points and a decrease of $30 \%$ from the baseline score with a decrease of at least one point on the rectal bleeding subscale or an absolute rectal bleeding score of 0 or 1 .

Clinical remission: defined as a Mayo score $\leq 2$ and no individual subscore $>1$.

Mucosal healing: defined as a mucosa subscore of $\leq 1$.

Disease activity: Mild 3-5; Moderate 6-10; Severe 11-12. 
Table 3 Ulcerative Colitis Endoscopic Index of Severity (UCEIS) $)^{35}$

\begin{tabular}{|c|c|c|}
\hline Descriptor (score most severe lesions) & Likert scale & Definition \\
\hline \multirow[t]{3}{*}{ Vascular pattern* } & Normal (1) & $\begin{array}{l}\text { Normal vascular pattern with arborisation of capillaries clearly defined, or with blurring or } \\
\text { patchy loss of capillary margins }\end{array}$ \\
\hline & Patchy obliteration (2) & Patchy obliteration of vascular pattern \\
\hline & Obliterated (3) & Complete obliteration of vascular pattern \\
\hline \multirow[t]{4}{*}{ Bleeding* } & None (1) & No visible blood \\
\hline & Mucosal (2) & $\begin{array}{l}\text { Some spots or streaks of coagulated blood on the surface of the mucosa ahead of the scope, } \\
\text { that can be washed away }\end{array}$ \\
\hline & Luminal mild (3) & Some free liquid blood in the lumen \\
\hline & Luminal moderate or severe (4) & $\begin{array}{l}\text { Frank blood in the lumen ahead of endoscope or visible oozing from mucosa after washing } \\
\text { intraluminal blood, or visible oozing from a haemorrhagic mucosa }\end{array}$ \\
\hline \multirow[t]{4}{*}{ Erosions and ulcers* } & None (1) & Normal mucosa, no visible erosions or ulcers \\
\hline & Erosions (2) & Tiny $(\leq 5 \mathrm{~mm}$ ) defects in the mucosa, of a white or yellow colour with a flat edge \\
\hline & Superficial ulcer (3) & $\begin{array}{l}\text { Larger }(>5 \mathrm{~mm}) \text { defects in the mucosa, which are discrete fibrin-covered ulcers when compared } \\
\text { with erosions, but remain superficial }\end{array}$ \\
\hline & Deep ulcer (4) & Deeper excavated defects in the mucosa, with a slightly raised edge \\
\hline
\end{tabular}

UCEIS score=sum of all three descriptors in the worst affected area of the colon visible at endoscopy.

Remission, score $\leq 1$.

* These three features account for $90 \%$ of variability in assessment of severity.

with moderately active UC had a better response to $4.8 \mathrm{~g} /$ day than $2.4 \mathrm{~g} /$ day $(72 \%$ vs $59 \%, \mathrm{p}=0.036) .{ }^{62}$ In the ASCEND III trial, 772 patients with moderately active UC were randomised to receive $2.4 \mathrm{~g} /$ day or $4.8 \mathrm{~g} /$ day mesalazine. ${ }^{63}$ Despite the large numbers in the study, there was no difference in the primary end-point of treatment success (complete clinical remission or partial response). There was a small but significant difference in remission, with $43 \%$ of patients on $4.8 \mathrm{~g}$ /day versus $35 \%$ of those on $2.4 \mathrm{~g} /$ day achieving remission at 6 weeks. Subgroup analysis showed that patients who had received steroid, oral 5-ASA and rectal therapies were more likely to respond to the higher dose. ${ }^{63}$ $4.8 \mathrm{~g} /$ day 5 -ASA is associated with more rapid symptom resolution than $2.4 \mathrm{~g} / \mathrm{day},{ }^{64}$ and higher rates of mucosal healing at 3 and 6 weeks. ${ }^{65}$ Similarly, the 5-ASA MMX trials also showed that $4.8 \mathrm{~g} /$ day was more effective than $2.4 \mathrm{~g} /$ day in the subpopulation with prior exposure to 5-ASA, and in those who had an incomplete response to 8 weeks of 5-ASA MMX at $2.4 \mathrm{~g} /$ day. ${ }^{66}$

A post-hoc analysis of the ASCEND I and II data also showed that there was greater mucosal healing in the $4.8 \mathrm{~g} /$ day group compared with $2.4 \mathrm{~g} /$ day. ${ }^{65}$ There is also evidence of the effect of escalating doses of 5-ASA from the DEAR trial. ${ }^{67}$ In this study patients with quiescent UC but with a raised faecal calprotectin $>50 \mu \mathrm{g} / \mathrm{g}$ had their current 5-ASA dose increased (from $1.2 \mathrm{~g}$ (or nil) to $2.4 \mathrm{~g}$, or from $2.4 \mathrm{~g}$ to $4.8 \mathrm{~g} /$ day). Compared with a control group there was a significant increase in patients remaining in clinical remission, and with a faecal calprotectin $<50 \mu \mathrm{g} / \mathrm{g}$ at 6 weeks. Oral 5-ASA is no more effective than oral sulphasalazine, ${ }^{59}$ but may be better tolerated (RR for an adverse event $0.48,95 \%$ CI 0.36 to 0.63$){ }^{57}$

Following commencement of 5-ASA, 10-30\% of patients are in symptomatic remission at week 2, 30-45\% by week 4 and $35-50 \%$ by week $8 .^{68-71}$ High-dose 5-ASA should not be used routinely, but patients with symptoms that are failing to settle should have their dose increased.

\subsubsection{Oral and enema 5-ASA}

All UC patients (including those with extensive disease) should be offered a combination of oral and enema 5-ASA, and those with incomplete response to oral 5-ASA should have topical therapy added. The combination of oral and topical 5-ASA therapy is superior to monotherapy even in patients with pancolitis. ${ }^{72} 73$ A meta-analysis of four studies in active UC confirmed a relative risk of no remission of 0.65 (95\% CI 0.47 to 0.91 ) for combined oral and topical therapy over oral 5-ASA alone for induction of remission. ${ }^{74}$ A small study in 18 UC patients with frequent relapses on standard dose oral 5-ASA showed the benefits of high-dose 5-ASA combined with 5-ASA enemas. ${ }^{75}$ Despite the evident benefits of enema therapy, the practical difficulty patients have in administering and retaining enemas remains a major obstacle, and support and education in this area is much needed.

\subsubsection{Nephrotoxicity}

5-ASA therapy may be associated with renal complications. ${ }^{7677}$ It is important to obtain baseline renal function, as renal disease may also be a primary complication of IBD itself. Renal manifestations of IBD include nephrolithiasis, urinary obstruction, fistulisation, glomerular disease, protein-losing nephropathy, secondary amyloidosis and renal failure. ${ }^{76} 78$ Nephrotic syndrome due to minimal-change nephropathy has been reported in a patient taking sulfasalazine, resolving with drug withdrawal and high-dose oral corticosteroids. ${ }^{79}$ Allergic reactions to sulfasalazine have recurred on challenge 5-ASA. ${ }^{80}$

Table 4 Modified Mayo Endoscopic Score ${ }^{37}$

\begin{tabular}{|c|c|c|c|c|c|}
\hline & Ascending & Transverse & Descending & Sigmoid & Rectum \\
\hline $\begin{array}{l}\text { Mayo endoscopic subscore: evaluated macroscopically at most severely inflamed part per segment } \\
\text { (score } 0-3 \text { : see table } 2 \text { ) }\end{array}$ & a & $b$ & c & $\mathrm{d}$ & e \\
\hline Maximal Extent in decimetres (during withdrawal) & \multicolumn{5}{|l|}{$\mathrm{ME}$} \\
\hline Extended Modified Score (EMS) & \multicolumn{5}{|c|}{$E M S=(a+b+c+d+e) \times M E$} \\
\hline Modified Mayo Endoscopic Score (MMES) & \multicolumn{5}{|c|}{ MMES=EMS/(number of segments with score $>0$ ) } \\
\hline
\end{tabular}


Statement 2. We suggest that symptomatic remission combined with mucosal healing should be the target of medical therapy in ulcerative colitis (GRADE: weak recommendation, very low-quality evidence. Agreement: 95.7\%).
Tubulointerstitial nephritis is a rare idiosyncratic reaction to 5-ASA therapy.

A UK study evaluated 151 patients with renal impairment associated with 5-ASA use. ${ }^{81}$ The temporal association with the drug, improvement on 5-ASA withdrawal (in 30\% of the cases) and the recurrent renal damage on re-challenge in five patients was consistent with a true relationship, and a suggestive genetic association in the HLA region $\left(p=1 \times 10^{-7}\right)$ was identified. Permanent renal replacement therapy was required in 15 patients. The median time on 5-ASA before renal impairment was 3 years (95\% CI 2.3 to 3.7$)$, and regular monitoring of renal function was uncommon. The frequency of 5-ASA nephrotoxicity has been estimated at 1 in 4000 patient years. ${ }^{82}{ }^{83}$ Given the unpredictable nature of this occurrence, it is suggested that patients on long-term 5-ASA therapy should have renal function checked, including eGFR before starting, after 2-3 months, and then annually long-term, although there are no data to support a particular surveillance interval. Those with impaired renal function should be monitored more closely.

\subsection{Corticosteroids in mild to moderate UC}

Prednisolone is superior to 5-ASA for induction of remission in UC, ${ }^{84}$ but has significant side effects and should be reserved for patients with failure of response or who are intolerant to oral and/or rectal 5-ASA (see Section 3.7: on Corticosteroids in moderate to severe UC).

\subsubsection{Budesonide MMX}

Randomised controlled trials have shown that oral budesonide MMX 9 mg daily is significantly more effective than placebo and can induce remission in mild to moderate UC, being as effective as 5-ASA. ${ }^{86-88}$ The CORE I and CORE II studies compared oral budesonide MMX $9 \mathrm{mg} /$ day and $6 \mathrm{mg}$ /day with placebo in patients with mild to moderate left-sided and extensive UC. ${ }^{87}$ Additional control groups were included (Asacol $2.4 \mathrm{~g} /$ day (CORE I) and Entocort EC $9 \mathrm{mg}$ (CORE II)), but the studies were not powered to demonstrate differences between budesonide MMX and these controls. In a pooled analysis of both trials, the week 8 combined clinical and endoscopic remission rates were $17.7 \%$ for budesonide MMX $9 \mathrm{mg}$ versus $6.2 \%$ for placebo $(\mathrm{p}=0.0002)$. A Cochrane systematic review concluded that the quality of evidence was moderate, and benefit was not demonstrated clearly for extensive UC but was significant for those with left-sided disease. ${ }^{89}$ Endoscopic healing rates were $27.6 \%$

Statement 3. We recommend that mild to moderate ulcerative colitis should be managed with oral 5-ASA 2-3 g/day (GRADE: strong recommendation, high-quality evidence). We recommend the addition of 5-ASA enemas, rather than oral treatment alone (GRADE: strong recommendation, high-quality evidence. Agreement: 95.6\%).

Statement 4. We suggest that ulcerative colitis patients flaring on 5-ASA therapy should receive dose escalation to 4-4.8 g/day orally alongside 5-ASA enemas (GRADE: weak recommendation, low-quality evidence. Agreement: $82.2 \%$ ).
Statement 5 . We recommend that ulcerative colitis patients treated with 5-ASA should be monitored for the development of nephrotoxicity, with baseline renal function, repeated after 2-3 months, and then annually (GRADE: strong recommendation, very low-quality evidence. Agreement: 90.9\%).

versus $17.1 \%(p=0.009)$ for budesonide MMX and placebo, respectively. ${ }^{90}$ The $6 \mathrm{mg}$ dose was not significantly better than placebo. Another placebo-controlled trial of budesonide MMX in mild to moderately active UC showed significant benefit in clinical, endoscopic and histological remission. ${ }^{91}$ Although no adequately powered comparative trials between budesonide MMX and conventional corticosteroids have been conducted to date, budesonide MMX may be considered as an alternative to conventional corticosteroids in patients with mild-moderate UC and failure of response to 5-ASA therapy. ${ }^{92}$

Budesonide has a lower rate of systemic adverse effects than conventional corticosteroids (33\% vs 55\%), and is not associated with adrenal suppression or a significant reduction in bone mineral density. ${ }^{93} 94$ Ileal-release budesonide does not induce remission in mild-moderately active UC and was in fact inferior to placebo and 5-ASA in two studies and a systematic review. $^{899395}$

\subsubsection{Beclomethasone dipropionate}

Oral beclomethasone dipropionate is a second-generation corticosteroid and may also be an alternative to conventional corticosteroids. In a randomised controlled trial in 282 patients, oral beclomethasone dipropionate $5 \mathrm{mg}$ daily for 4 weeks, then alternate weekly for a further 4 weeks was shown to be non-inferior to prednisolone in efficacy, but there was also no difference in the co-primary end-point of steroid-related adverse events and reduction in morning cortisol below $150 \mathrm{nmol} / \mathrm{L} .{ }^{96}$ In patients with active left-sided or extensive UC, oral beclomethasone dipropionate $5 \mathrm{mg} /$ day has been demonstrated as equivalent to $2.4 \mathrm{~g} 5$-ASA, ${ }^{97}$ and has been shown as more effective when added to 5-ASA compared with 5-ASA alone. ${ }^{98}$

Although more expensive, topically-acting oral corticosteroids are an alternative to prednisolone in UC patients with mild to moderate disease and 5-ASA failure.

\subsection{Corticosteroids in moderate to severe UC}

Oral corticosteroids are effective for the induction of remission in patients with moderate to severe ulcerative colitis refractory to sulfasalazine or 5-ASA, and in patients who have responded to initial treatment with intravenous corticosteroids following hospitalisation for acute severe disease. ${ }^{84} 85$

In a meta-analysis of five randomised controlled trials, corticosteroids were superior to placebo for induction of remission in

Statement 6. We recommend that patients with mild to moderate ulcerative colitis in whom 5-ASA induction therapy fails or is not tolerated should be treated with oral prednisolone (GRADE: strong recommendation, high-quality evidence). We recommend that topically-acting oral corticosteroids such as budesonide MMX (GRADE: strong recommendation, moderatequality evidence) and we suggest that beclomethasone dipropionate (GRADE: weak recommendation, moderate-quality evidence) can be used as alternative treatments for those wishing to avoid systemic corticosteroids (Agreement: 93.2\%). 
Statement 7. We recommend that moderate to severe ulcerative colitis should be treated with oral corticosteroids such as prednisolone $40 \mathrm{mg}$ daily weaning over $6-8$ weeks (GRADE: strong recommendation, high-quality evidence. Agreement: $100 \%)$

UC (RR of no remission, $0.65 ; 95 \%$ CI 0.45 to 0.93$).{ }^{99}$ Although the optimal dose and regimen for systemic corticosteroids in UC is uncertain, the current $40 \mathrm{mg}$ dose recommendation is based on Baron's studies where $40 \mathrm{mg}$ was more effective than $20 \mathrm{mg} /$ day. ${ }^{100}$ There is no evidence of benefit with doses higher than $40-60 \mathrm{mg} / \mathrm{day}^{99}{ }^{99}$ and doses above $40 \mathrm{mg}$ may be associated with increased adverse effects. ${ }^{100}$ Approximately 50\% of patients experience short-term corticosteroid-related adverse events such as acne, oedema, sleep and mood disturbance, glucose intolerance and dyspepsia. ${ }^{99} 100$ Single daily dosing is as effective as split-dosing and causes less adrenal suppression. ${ }^{101}$ The dose should be tapered over 6-8 weeks.

\subsubsection{Failure of oral corticosteroids}

There is variation in the definition of severe ulcerative colitis. The Mayo score or Disease Activity Index ${ }^{32}$ does not include systemic symptoms (fever, anaemia or abnormal inflammatory markers), whereas the Truelove and Witts criteria for severe disease $^{102}$ specify one or more of these. Patients with systemic symptoms of fever, severe pain, significant anaemia, or those who are generally unwell and not tolerating their symptoms should be admitted for inpatient management (see Section 3.12: on Acute severe ulcerative colitis). Prolonging treatment with high-dose oral corticosteroids has a diminishing chance of achieving remission, and of those who do respond, there will be many who become corticosteroid-dependent (22\% at 1 year in a study from the pre-biologic era $\left.{ }^{103}\right)$. There is however increasing risk of infective, metabolic and surgical complications for deteriorating patients who may require emergency admission and colectomy. ${ }^{104} 105$ There is no evidence from a meta-regression analysis that doses above $60 \mathrm{mg}$ of methylprednisolone reduce colectomy rates in severe UC. ${ }^{106}$ Although there are few data on the time-course of response to oral corticosteroids, a study comparing oral prednisolone with prednisolone metasulphobenzoate showed that improvements in clinical and endoscopic disease activity may be seen within 2 weeks of treatment with oral prednisolone $40 \mathrm{mg} / \mathrm{day},{ }^{107}$ so patients not responding after 2 weeks should be considered for treatment escalation to biologics or admission to hospital, depending on how systemically unwell they are.

\subsection{5-ASA maintenance therapy for UC}

Maintenance 5-ASA therapy is advocated to decrease the risk and frequency of flares. The most recent Cochrane analysis showed a

Statement 8 . We recommend that oral 5-ASA should be the standard maintenance medical therapy in ulcerative colitis (GRADE: strong recommendation, high-quality evidence). We recommend that the choice of formulation should consider patient preference, likely adherence and cost. Once daily dosing is effective (GRADE: strong recommendation, highquality evidence) and may improve adherence (Agreement: $100 \%)$.
Statement 9. We recommend that ulcerative colitis patients on maintenance therapy with high-dose mesalazine, who required two or more courses of corticosteroids in the past year, or who become corticosteroid-dependent or refractory, require treatment escalation with thiopurine (GRADE: strong recommendation, moderate-quality evidence), anti-TNF therapy (GRADE: strong recommendation, high-quality evidence), vedolizumab (GRADE: strong recommendation, high-quality evidence) or tofacitinib (GRADE: strong recommendation, high-quality evidence). The choice of drug should be determined by clinical factors, patient choice, cost, likely adherence and local infusion capacity (Agreement: 96.6\%).

trend towards greater efficacy with higher doses ( $2 \mathrm{~g}$ or more). ${ }^{108}$ Rectal 5-ASA is also an effective maintenance therapy for distal ulcerative colitis. ${ }^{109} 110$ There are randomised controlled trials evaluating once daily dosing with all the major 5-ASA formulations, and systematic reviews confirm that once daily dosing is as effective as divided dosing regimens. ${ }^{111-114}$ In community surveys, 5-ASA adherence is a major problem and some studies suggest that simplified dosing regimens are associated with better adherence. ${ }^{115}$ Therefore, once daily dosing should be considered as a standard dosing regimen for all 5-ASA use.

In an evaluation of data from Cochrane analyses there were no differences between the various 5-ASA formulations in terms of efficacy, ${ }^{116}$ confirmed in the most recent Cochrane analysis. ${ }^{108}$ There is little to choose between the different formulations of 5-ASA in terms of efficacy, and the best drug should be selected taking into account patient preference for formulation (for instance granules or tablets, tablet size and number required daily), in order to maximise treatment adherence ${ }^{117}$ and considering cost.

\subsection{Treatment options for UC patients after 5-ASA failure 3.9.1 Thiopurines}

While studies vary in quality, meta-analyses consistently report a benefit of thiopurines over placebo for the maintenance of steroid-induced remission in UC but not for induction of remission. Meta-analysis of three randomised controlled thiopurine maintenance studies favours thiopurines over placebo (RR 0.6, $95 \%$ CI 0.37 to 0.95$).{ }^{118}$ In a further meta-analysis, OR was 2.59 (95\% CI 1.26 to 5.3), absolute risk reduction was $23 \%$ and the number-needed-to-treat (NNT) to prevent one recurrence was five. ${ }^{119}$ A recent Cochrane review included 232 patients from four maintenance studies of azathioprine versus placebo and showed a benefit of azathioprine over placebo (44\% vs $65 \%$ failure, respectively, RR 0.68 , 95\% CI 0.54 to 0.86$).{ }^{120}$ The side effects of thiopurines, both short- and long-term, should be considered (see Section 5.2.2 on Drug management: thiopurines) in choice of therapy, and also in decisions about duration of therapy, particularly in older patients (see Section 5.2.7.1 Stopping thiopurines).

\subsubsection{Infliximab}

In the ACT1 and ACT2 clinical trials, UC patients with active disease despite corticosteroids and/or thiopurines (and/or 5-ASA for ACT2 only) were treated with 5 or $10 \mathrm{mg} / \mathrm{kg}$ infliximab or placebo at weeks 0, 2 and 6, and followed to week 54 (ACT1) or week 30 (ACT2). Clinical response at week 8 was similar in both active doses, and pooled data from both trials were $67 \%$ for $5 \mathrm{mg} / \mathrm{kg}$ versus $33 \%$ for placebo. ${ }^{121}$ At week 30 combined 
clinical remission rates were $30 \%$ for $5 \mathrm{mg} / \mathrm{kg}$ ( $13 \%$ for placebo), with very similar remission rates sustained to week 54 in ACT1. Corticosteroid-free remission rates were $22 \%$ for $5 \mathrm{mg} / \mathrm{kg}$ at week 30, sustained to week 54 in ACT1. In the UC SUCCESS study, patients in whom corticosteroid therapy had failed and who were receiving infliximab and azathioprine combination therapy $(40 \%)$ had significantly higher remission rates at week 16 , compared with infliximab alone (22\%). ${ }^{122} 10 \%$ of these patients had previously received immunomodulators, but not anti-TNF therapy. Cohort studies also show benefit of using infliximab for patients with corticosteroid-dependent UC, with steroid-free remission rates of $47 \%$ at 1 year, $77 \%$ colectomy-free at median 41.5 months, and better outcomes for those on combination therapy with thiopurines, and also in those who were thiopurine-naïve initially. ${ }^{123124}$ Real-life experience of infliximab treatment in UC patients where conventional therapy had failed showed primary response rates vary from $67 \%$ (in a study from Leuven, starting infliximab prior to 2006 and including patients recruited to the ACT1 study), ${ }^{125}$ to $78 \%$ in a French multicentre study of patients treated from 2000 to $2009 .^{126}$

\subsubsection{Adalimumab}

The ULTRA1 and ULTRA2 clinical trials ${ }^{127128}$ showed that adalimumab $160 \mathrm{mg}$ at week $0,80 \mathrm{mg}$ at week 2 , followed by $40 \mathrm{mg}$ fortnightly achieved remission at week 8 in 19\% (placebo 9\%) in ULTRA1 and 21\% (placebo 11\%) in the ULTRA2 study in patients naïve to anti-TNF therapy. The ULTRA2 maintenance study showed clinical remission rates at week 52 of 22\% (12\% placebo) in the anti-TNF naïve subgroup. Corticosteroid-free remission at week 52 in the same subgroup was 14\% (placebo $6 \%$ ). In the open-label extension study (ULTRA3), 25\% remained in clinical remission on fortnightly or weekly adalimumab at 4 years after initial enrolment. ${ }^{129}$ Real-world data from the USA on the use of infliximab and adalimumab in UC patients naïve to anti-TNF therapy suggests comparable efficacy in the treatment of moderate to severe disease. ${ }^{130131}$

\subsubsection{Golimumab}

The PURSUIT SC trial was an induction trial of golimumab, which included a dose-finding phase. ${ }^{132} 1064$ patients with UC that had failed to respond to either 5-ASA, oral corticosteroids, azathioprine or mercaptopurine, or who were steroid-dependent, were enrolled. All patients were anti-TNF naïve. 42.8\% were receiving steroids at baseline, and a third were on thiopurines. Clinical response was achieved at week 6 in $51.0 \%$ on $200 \mathrm{mg} / 100 \mathrm{mg}, 54.9 \%$ at $400 \mathrm{mg} / 200 \mathrm{mg}$, both significantly better $(\mathrm{p}<0.0001)$ versus placebo response rate of $30.3 \%$. Clinical remission at week 6 was $17.8 \%(200 \mathrm{mg} / 100 \mathrm{mg}), 17.9 \%$ $400 \mathrm{mg} / 200 \mathrm{mg}, \mathrm{p}<0.0001$ versus placebo $(6.4 \%)$. Both clinical response and remission at week 6 , correlated with drug levels. Real-world observational studies corroborate with PURSUIT study observations. ${ }^{133}$

Network meta-analyses comparing the different anti-TNF agents in UC present conflicting data on the relative efficacy of infliximab, adalimumab and golimumab. ${ }^{134-137}$

\subsubsection{Vedolizumab}

In the GEMINI I clinical trial of vedolizumab in active UC, patients were enrolled to a randomised cohort. 225 patients received intravenous vedolizumab $300 \mathrm{mg}$ at day 1 and 15, 149 patients received placebo and 521 patients were randomised to open-label vedolizumab in order to fulfil sample size requirements for the continuation maintenance study. ${ }^{138} 139$ Of the
anti-TNF naïve patients in the randomised induction phase, clinical response at 6 weeks was seen in $26.3 \%$ of 76 patients on placebo versus $53.1 \%$ of 130 patients on vedolizumab, an estimated $26.4 \%$ difference (95\% CI $12.4 \%$ to $40.4 \%$ ). Clinical remission at 6 weeks was seen in $6.6 \%$ on placebo and $23.1 \%$ on vedolizumab, an estimated difference of $15.5 \%(95 \% \mathrm{CI}$ $5.1 \%$ to $25.9 \%$ ). Of the randomised responders who entered the maintenance phase (including those from the open-label induction cohort) and who had been anti-TNF naïve at entry, the durable clinical response rate (response at both weeks 6 and 52) was $26.6 \%$ for the 79 patients on placebo compared with $60.7 \%$ for the 145 patients given vedolizumab (either 4 -weekly or 8-weekly). The estimated difference from placebo was $34.3 \%$ (95\% CI $20.7 \%$ to $47.8 \%$ ). The clinical remission rate at week 52 for the placebo group was $19.0 \%$, and $46.9 \%$ for the combined vedolizumab group, an estimated difference from placebo of $28.0 \%$ (95\% CI $14.9 \%$ to $41.1 \%$ ). There were no clinically important differences in safety between the vedolizumab and placebo groups during the maintenance study. ${ }^{139}$

A recently published retrospective study from the VICTORY consortium analysed safety and efficacy data from 321 UC patients receiving vedolizumab therapy $(71 \%$ of whom had previously been treated with anti-TNF therapy). ${ }^{140}$ Primary effectiveness outcomes were cumulative rates of clinical remission (defined as resolution of all UC-related symptoms on physician's global assessment), endoscopic remission (defined as a Mayo endoscopic subscore of 0 ), corticosteroid-free remission and deep remission (clinical remission and endoscopic remission). In recognition of potential attrition bias, variability in follow-up and impact of right censoring, imputation analyses calculated 12 month rates as $20 \%, 17 \%, 15 \%$ and $14 \%$ respectively. On multivariable analyses, prior exposure to anti-TNF therapy was associated with a reduced probability of achieving clinical remission (HR $0.53,95 \%$ CI 0.38 to 0.75 ) and endoscopic remission (HR $0.51,95 \%$ CI 0.29 to 0.88 ).

Until recently there has been limited reporting of vedolizumab therapy outcomes for anti-TNF naïve patients with UC. However, a multicentre European retrospective study of 184 patients (50 Crohn's disease and 134 UC patients) showed a greater efficacy than that reported in anti-TNF experienced patients with response, clinical remission and steroid-free remission rates at week 14 of $79.1 \%, 39.5 \%$ and $36.6 \%$, respectively, in UC. ${ }^{141}$ The VARSITY trial has now been reported in abstract form and is the first head-to-head comparison of two biologic agents in IBD. ${ }^{142}$ Seven hundred and sixty-nine patients with moderately to severely active UC who had failed conventional therapies were randomised to intravenous vedolizumab $300 \mathrm{mg}$ at weeks 0,2 and 6 and then every 8 weeks, versus adalimumab subcutaneously $160 \mathrm{mg}$ at week $0,80 \mathrm{mg}$ at week 2 , and then $40 \mathrm{mg}$ fortnightly, in a double-blind, double-dummy trial. Dose escalation was not permitted for either group. Recruitment of patients with previous anti-TNF therapy use was capped at 25\% of the trial population. At 52 weeks, the primary end-point of clinical remission (a complete Mayo score $\leq 2$ with no subscore $>1$ ) was achieved in $31.3 \%$ on vedolizumab versus $22.5 \%$ on adalimumab $(\mathrm{p}=0.006)$. Mucosal healing (Mayo endoscopic subscore $\leq 1$ ) was $39.7 \%$ and $27.7 \%$, respectively $(p=0.0005)$. Corticosteroid-free remission rates (in those on steroids at baseline) at week 52 were not significantly different between the vedolizumab and adalimumab groups. Although no similar data are available for comparison with infliximab, these data provide support for vedolizumab as a first-line biologic option for UC failing conventional therapy. 


\subsubsection{Tofacitinib}

A phase 2 dose-ranging induction study of the Janus kinase (JAK) inhibitor tofacitinib in patients with endoscopically confirmed active UC showed increased rates of clinical and endoscopic response and remission after 8 weeks of treatment that reached statistical significance for patients in the higher dosing arms of $10 \mathrm{mg}$ and $15 \mathrm{mg}$ oral twice daily. ${ }^{143}$ The OCTAVE- 1 and OCTAVE- 2 phase 3 induction studies confirmed the efficacy of a dose of $10 \mathrm{mg}$ twice daily as induction therapy for active colitis. ${ }^{144}$ In these studies, the primary end-point of remission was defined clinically and endoscopically at week 8 by a total Mayo score $\leq 2$, with no individual subscore $>1$ and a rectal bleeding subscore of 0 . This was achieved by $18.5 \%$ and $16.6 \%$ of patients on active treatment in OCTAVE- 1 and OCTAVE-2 versus $8.2 \%$ and $3.6 \%$ of patients on placebo $(p=0.007$ and $\mathrm{p}<0.001$, respectively). For patients who had not previously received anti-TNF therapy, remission at 8 weeks on placebo and tofacitinib was $12.5 \%$ and $23.7 \%$ respectively (difference $11.2 \%$; $95 \%$ CI $2.6 \%$ to $17.9 \%$ ). Clinical responders to induction therapy (where response was defined as a decrease from baseline in the total Mayo score of $\geq 3$ points and $>30 \%$ with a rectal bleeding score decrease of at least one or a rectal bleeding subscore of 0 or 1 ) were eligible for enrolment in the maintenance OCTAVE-SUSTAIN trial. Of patients randomised to tofacitinib pooled from both trials, $57.6 \%(521 / 905)$ had a clinical response at week 8 . This included both anti-TNF naïve and anti-TNF exposed patients. They were randomised 1:1:1 to oral tofacitinib $5 \mathrm{mg}$ twice daily, $10 \mathrm{mg}$ twice daily or placebo. After 1 year, remission was achieved in $34.3 \%$ in the tofacitinib $5 \mathrm{mg}$ group, $40.6 \%$ in the tofacitinib $10 \mathrm{mg}$ group and $11.1 \%$ in the placebo group $(\mathrm{p}<0.001$ for both treatment arms vs placebo).

In the OCTAVE open-label follow-on study, patients who responded to induction therapy with $10 \mathrm{mg}$ twice daily, but were then randomised to placebo in the OCTAVE-SUSTAIN study and then relapsed, were retreated with $10 \mathrm{mg}$ twice daily open-label. ${ }^{145}$ Of these, $75.8 \%$ responded at month 2 and $67.5 \%$ at 1 year, showing that retreatment is effective after a break in therapy. The OCTAVE open-label study also showed that patients receiving $5 \mathrm{mg}$ twice daily in the OCTAVE-SUSTAIN study who relapsed and were then treated with $10 \mathrm{mg}$ twice daily open-label had a response rate of $58.6 \%$ at month 2 and $68.8 \%$ at 1 year. ${ }^{146}$

Although total adverse events did not appear to differ significantly between treatment and placebo groups, there was an increased risk of infections seen in tofacitinib treated patients during both induction and maintenance phases. In particular, herpes zoster occurred more often on active treatment, a finding consistent with prior experience of use of tofacitinib in patients with rheumatoid arthritis and psoriasis. ${ }^{147} 148$ Zoster vaccination should be considered before starting therapy in those aged over 70 years and those over 50 years considered at particularly high risk (such as recurrent shingles). As a live vaccination it must not be given for 3 months after stopping biologics, and tofacitinib should not be started for 4 weeks after vaccination (see Section 5.2.1.3.2 Live vaccines). Recent data from an open-label study ${ }^{149}$ in rheumatoid arthritis patients (over 50 years with at least one cardiovascular risk factor), comparing tofacitinib $5 \mathrm{mg}$ or $10 \mathrm{mg}$ twice daily with TNF-inhibitor therapy, have shown a five-fold increase in pulmonary embolus for the group on $10 \mathrm{mg}$ twice daily tofacitinib compared with TNF inhibitor therapy), ${ }^{150}$ and at present the European Medicines Agency safety committee advises that the high dose should not be used in patients at increased risk of pulmonary embolus (heart failure, malignancy, impending/recent surgery, inherited coagulation disorders, previous thromboembolism, combined contraceptive therapy or
HRT). ${ }^{151}$ Further data are required on the risks for UC patients treated with JAK inhibitors.

No comparative data for tofacitinib with biologics are available, and NICE approval has been granted for the treatment of moderate to severely active UC where conventional treatments or biological agents have not been tolerated, or have failed. ${ }^{152}$ Tofacitinib has the advantages of oral administration and, as a small molecule, immunogenicity is not an issue. This has to be weighed against the side effect profiles and costs of different agents.

\subsubsection{Ustekinumab}

The UNIFI trial, recently presented in abstract form, investigated ustekinumab as induction and maintenance therapy in moderate to severely active UC in adults who had failed to respond or were intolerant to corticosteroids, immunomodulators, anti-TNF therapy (one or more) or vedolizumab. ${ }^{153} 154$ Patients were randomised $1: 1: 1$ to receive a single IV dose of placebo, $130 \mathrm{mg}$ ustekinumab, or approximately $6 \mathrm{mg} / \mathrm{kg}$ ustekinumab (weight-tiered dosing: patients weighing $\leq 55 \mathrm{~kg}$ received $260 \mathrm{mg}$; patients weighing $>55 \mathrm{~kg}$ and $\leq 85 \mathrm{~kg}$ received $390 \mathrm{mg}$; and patients weighing $>85 \mathrm{~kg}$ received $520 \mathrm{mg}$ ), as in the UNITI Crohn's studies. At week 8, 961 patients were evaluated. The primary end-point was clinical remission at week 8 (defined as a Mayo score $\leq 2$ points, with no individual subscore $>1$ ), and was achieved by $15.6 \%$ on $130 \mathrm{mg}$ ustekinumab, $15.5 \%$ on the approximately $6 \mathrm{mg} / \mathrm{kg}$ dose and $5.3 \%$ on placebo $(\mathrm{p}<0.001) .{ }^{153}$ Endoscopic healing (defined as Mayo endoscopy subscore of 0 or 1 ) was $26.3 \%, 27 \%$ and $13.8 \%$ in the three groups, respectively $(\mathrm{p}<0.001)$. Clinical response (decrease from baseline Mayo score of $\geq 30 \%$ and $\geq 3$ points, with either a decrease from baseline in the rectal bleeding subscore of $\geq 1$ or a rectal bleeding subscore of 0 or 1) was $51.3 \%, 61.8 \%$ and $31.3 \%$ in the three groups, respectively $(\mathrm{p}<0.001)$. Both active treatment groups had a significant improvement in IBDQ, mucosal healing (endoscopic healing as defined above), and histological healing (defined as $0-<5 \%$ neutrophils in epithelium, no crypt destruction and no erosions, ulcerations or granulations) was $20.3 \%, 18.4 \%$ and $8.9 \%$ respectively at week 8 . There was no difference in adverse events compared with placebo, with no malignancies, opportunistic infections or TB reported. In the maintenance continuation study, 523 patients with clinical response at week 8 were re-randomised to placebo, 8 -weekly or 12-weekly dosing, with week 44 remission rates of $24 \%, 38.4 \%$ and $43.8 \%$, respectively $(p=0.002$ for 8 -weekly and $p<0.001$ for 12 -weekly vs placebo). ${ }^{154}$ Effects were seen in both anti-TNF naïve and anti-TNF refractory patients, but numerical benefits of 8-weekly dosing (compared with 12-weekly) were restricted to the anti-TNF refractory population. The safety profile was consistent with that observed in Crohn's disease.

At the time of writing, ustekinumab does not have licensing or NICE approval for its use in UC. It should be noted that (unlike the UNITI studies in Crohn's disease and the GEMINI studies of vedolizumab in UC) the UNIFI study permitted inclusion of patients with failure of two classes of biologic (TNF inhibitors and vedolizumab) and results should be interpreted accordingly.

\subsubsection{Methotrexate}

Methotrexate has no role in the maintenance of remission in UC. A Cochrane review in 2015 of methotrexate use in comparison to placebo, 5-ASA, sulfasalazine and mercaptopurine does not 
support its use in maintenance of remission in UC. ${ }^{155}$ In a European double-blind randomised trial, 111 patients were allocated to $25 \mathrm{mg} /$ week parenteral methotrexate versus placebo alongside prednisolone for a flare of UC. ${ }^{156}$ Methotrexate was not superior to placebo $(31.7 \%$ vs $19.6 \%$ remission, $\mathrm{p}=0.15)$ for the primary end-point of steroid-free remission at week 16 (Mayo score $\leq 2$ with no item $>1$ and complete withdrawal of steroids and no use of another immunosuppressant (IS), anti-TNF therapy or colectomy). Clinical remission at week 16 (Mayo score $\leq 2$ with no item $>1$ ) was, however, significantly higher on methotrexate $(41.7 \%$ vs $23.5 \% \mathrm{p}=0.04)$. The MERIT study treated 179 patients with active UC with open-label methotrexate $25 \mathrm{mg}$ SC weekly, plus a tapering prednisolone course for 12 weeks. At 16 weeks there was a $51 \%$ response rate, and 84 patients were randomised to continue $25 \mathrm{mg}$ methotrexate SC or placebo. There was no difference in outcome at week 48, with $27 \%$ of those on methotrexate and $30 \%$ on placebo maintaining steroidfree clinical remission with no need for additional therapies. ${ }^{157}$

\subsubsection{Choice of immunosuppressive or biological therapy}

Patients with chronic active UC failing 5-ASA therapy have in the past been offered thiopurine therapy. As the range of alternatives grows and costs of biologics fall, there is strong justification for moving directly to other immunosuppressive drugs with less toxicity that may be easier to manage. Thiopurines still have a role as combination therapy and to reduce immunogenicity, but the therapeutic pyramid is changing rapidly.

Until the VARSITY study comparing vedolizumab with adalimumab, ${ }^{142}$ there had been no head-to-head studies comparing anti-TNF drugs, vedolizumab, tofacitinib and ustekinumab. Indirect means of comparison are much weaker and, at best, hypothesis-generating. Network meta-analyses have compared vedolizumab with anti-TNF drugs and show broadly similar benefits. ${ }^{158-160}$ In contrast, the VARSITY trial shows a significant benefit for vedolizumab compared with adalimumab $40 \mathrm{mg}$ every other week. ${ }^{142}$ Two network meta-analyses that include tofacitinib, one in abstract form, show comparable efficacy to anti-TNF agents and vedolizumab. ${ }^{161} 162$ The choice of which immunosuppressive agent to use depends on a number of factors (box 2). Patients may prefer to receive oral or subcutaneous therapy rather than intravenous therapy, although the latter may be preferred for patients where non-adherence may be an issue. Tofacitinib as an oral agent, with no concerns about immunogenicity, is an attractive choice, but with little real-world experience as yet. Gut-selective therapy may be considered safer because of concerns about risk of malignancy or infection; at present there is little long-term comparative safety data between

Box 2 Factors to consider in choosing a biologic, immunosuppressive or immunomodulator therapy

- Route of administration (oral, subcutaneous, intramuscular, intravenous)

- Speed of response to induction therapy (consider need for bridging therapy)

- Potential immunogenicity and need for combination therapy

- Side effects including cancer risk

- Persistence (continuing drug without loss of response after initial improvement)

- Availability of infusion facilities and therapeutic drug monitoring

- Overall cost (including drug delivery and monitoring)
Statement 10 . We recommend that vedolizumab can be used in the induction and maintenance of remission of ulcerative colitis in patients where anti-TNF treatment has failed (GRADE: strong recommendation, high-quality evidence. Agreement: 97.7\%).

vedolizumab and anti-TNF therapy to support this, although the long-term trial extension safety profile over 9 years from the GEMINI trials is very reassuring. ${ }^{163}$ The presence of significant extraintestinal manifestations may also be a consideration. Other practical issues are shown in box 2 .

\subsubsection{Therapeutic choice after anti-TNF failure}

Infliximab and other anti-TNF drugs have been used in UC for many years, and it is therefore important to consider alternatives for patients failing these drugs and who need to switch 'out of class' (see Sections 5.2.4.5: Common Disease Considerations, Primary non-response to anti-TNF therapy and 5.2.4.6: Common Disease Considerations, Secondary loss of response to anti-TNF therapy). It is important to note that surgery should always be discussed as an option in patients failing a therapeutic agent, particularly as there is generally a reduction in response to each successive immunosuppressive or biologic drug.

\subsubsection{Vedolizumab after anti-TNF therapy}

In the GEMINI I study of vedolizumab in active UC, 895 patients were enrolled and randomised responders were re-randomised to maintenance therapy or placebo. ${ }^{138} 367$ patients (41\%) had prior failure of anti-TNF therapy. Of these patients, clinical response at week 6 was 39\% for vedolizumab versus $20.6 \%$ for placebo (difference $18.1 \%, 95 \%$ CI $2.8 \%$ to $33.5 \%$ ). Of the randomised responders entering the maintenance phase who had prior anti-TNF failure, clinical remission at week 52 was $36.1 \%$ for vedolizumab (given either 8- or 4-weekly) versus $5.3 \%$ for placebo (difference $29.5 \%$, 95\% CI $12.8 \%$ to $46.1 \%)$. Vedolizumab is effective in patients with prior exposure to anti-TNF drugs, although benefits are larger in those with no prior exposure. ${ }^{139}$ Data on clinical efficacy and safety from prospectively followed cohorts on vedolizumab are now available. In a recently reported GETAID study, 294 patients with active IBD (121 with UC), with an inadequate or loss of response to conventional therapy or at least one anti-TNF agent, were treated with vedolizumab. ${ }^{164}$ Concomitant use of corticosteroids, thiopurines or methotrexate was permitted. Among patients with UC, 36\% were in steroid-free clinical remission and 50\% had clinical response at week 14 . In a review of safety data from vedolizumab trials, prior anti-TNF therapy was a risk factor for serious infection in UC patients (HR $1.99,95 \%$ CI 1.16 to 3.42 ; $\mathrm{p}=0.0122)$. Eighteen vedolizumab exposed patients $(<1 \%)$ were diagnosed with a malignancy including non-melanoma skin cancer, malignant melanoma, colon cancer, breast cancer, renal, liver and lung cancer; nearly all patients (except one with renal cancer) having had prior exposure to thiopurines and/or anti-TNF agents, making attribution difficult. ${ }^{165}$

\subsubsection{Tofacitinib after anti-TNF therapy}

In pooled data from the OCTAVE 1 and 2 trials, 589 patients had previously failed anti-TNF and had a week 8 clinical remission rate of $0.8 \%$ on placebo versus $11.4 \%$ on tofacitinib $10 \mathrm{mg}$ twice daily (difference $10.6 \%$, 95\% CI $7.3 \%$ to $13.9 \%$ ). ${ }^{166}$ Mucosal healing at week 8 (Mayo endoscopic subscore of 0 or 1 ) in patients with prior anti-TNF exposure was $6.5 \%$ on placebo versus $22.2 \%$ on tofacitinib $10 \mathrm{mg}$ twice daily (difference 
Statement 11 . We recommend that tofacitinib can be used in the induction and maintenance of remission of ulcerative colitis in patients where anti-TNF treatment has failed (GRADE: strong recommendation, high-quality evidence. Agreement: 91.1\%).

$15.7 \%$, 95\% CI $10.0 \%$ to $21.4 \%$ ). In OCTAVE-SUSTAIN, week 52 remission data for placebo, tofacitinib $5 \mathrm{mg}$ twice daily and $10 \mathrm{mg}$ twice daily in patients with prior anti-TNF failure were $11.2 \%, 24.1 \%$ and $36.6 \%$, respectively, for clinical remission and $12.4 \%, 30.1 \%$ and $39.8 \%$, respectively, for mucosal healing.

\subsection{Proctitis}

\subsubsection{5-ASA suppositories in proctitis}

When treating mild to moderate proctitis, first-line therapy should be 5-ASA suppositories. These medications achieve much higher mucosal concentrations of the drug and work faster and better than oral 5-ASA monotherapy in disease which is confined to the rectum. ${ }^{167}$ Higher response rates are achieved if topical therapies are combined with oral 5-ASA, ${ }^{168}$ thus patients not responding fully to suppositories should have early addition of oral therapy. Suppositories are preferred to enemas for proctitis as they deliver the drug specifically to the rectum (enemas tend to pool higher up in the sigmoid) and are also better tolerated/retained, as shown in a study comparing 5 -ASA suppositories with hydrocortisone foam enemas. ${ }^{169}$ Topical 5-ASA is more effective than topical corticosteroid. ${ }^{170}$ A Cochrane review did not show a dose-response relationship for rectal therapy (although this review assessed treatment of distal colitis, which included both proctitis and left-sided colitis up to the splenic flexure). ${ }^{171} \mathrm{~A}$ further review evaluating mucosal healing showed a dose-response relationship for oral but not rectal 5-ASA (again evaluating proctitis along with distal colitis). ${ }^{172}$ Divided doses are less convenient for patients. A study comparing $1 \mathrm{~g}$ 5-ASA suppository daily versus $500 \mathrm{mg}$ three times daily showed more convenience and similar efficacy (except for mucosal healing) for the once daily dose, ${ }^{173}$ and a further study showed no difference between $1 \mathrm{~g}$ daily and $500 \mathrm{mg}$ twice daily. ${ }^{174}$ Administering this dose at bedtime is practical for most patients, allowing the suppository to be retained for the longest possible time. ${ }^{171}$

\subsubsection{Maintenance therapy for proctitis}

Many patients respond promptly to 5-ASA suppositories, and if they have infrequent flares, are happy to start suppositories when they begin to flare and not take regular maintenance therapy. This is quite safe as the colorectal cancer risk in proctitis is similar to the general population. For many, however, regular preventive treatment is needed, and 5-ASA suppositories are the most effective maintenance therapy for proctitis. ${ }^{109} 175$ In longterm treatment, however, this needs to be weighed against the importance of adherence, and some patients may choose not to use long-term rectal therapy and prefer oral therapy. It is worth noting that alternate night or every third night suppositories do not appear to reduce the rate of maintenance of remission substantially. ${ }^{170}$

Statement 12 . We recommend that mild or moderately active ulcerative proctitis should be treated with a $1 \mathrm{~g} 5$-ASA suppository (GRADE: strong recommendation, high-quality evidence. Agreement: 100\%).
Statement 13. We suggest that patients with ulcerative proctitis who do not respond or are intolerant to 5-ASA suppositories and oral 5-ASA may be switched to corticosteroid suppositories (GRADE: weak recommendation, low-quality evidence. Agreement: 84.8\%).

\subsubsection{Corticosteroid suppositories in proctitis}

Steroid suppositories are also effective in proctitis but not as effective as 5-ASA and so their use should be confined to those who either do not tolerate or do not respond to 5-ASA. ${ }^{171} 176$ Oral 5-ASA should be continued if possible. For those not responding to 5-ASA, the addition of a prednisolone $5 \mathrm{mg}$ suppository in the morning while continuing 5-ASA suppositories at bedtime is worth trying. A novel budesonide suppository was studied in a four-group, prospective, double-blind, double-dummy randomised trial to treat UC proctitis with budesonide $2 \mathrm{mg}$, budesonide $4 \mathrm{mg}, 5$-ASA $1 \mathrm{~g}$, or budesonide $2 \mathrm{mg}$ plus 5-ASA $1 \mathrm{~g} .{ }^{177}$ The 8 -week study conducted in Germany, Russia and the UK assessed a primary end-point of resolution of clinical symptoms for three consecutive days (score of 0 for rectal bleeding and stool frequency on modified UCDAI). Budesonide $4 \mathrm{mg}$ was more effective than $2 \mathrm{mg}$, but no different from 5 -ASA $1 \mathrm{~g}$ or the combination of budesonide $2 \mathrm{mg}$ and 5-ASA $1 \mathrm{~g}$.

\subsubsection{Treatment options in refractory proctitis}

A proctitis management flowchart is shown in figure 1. In severe or refractory proctitis, it is important to ensure that conventional therapy has been delivered appropriately (with assessment of adherence) and that the diagnosis is correct. Proximal constipation is common and may contribute to symptoms and poor response to therapy, as may co-existing irritable bowel syndrome. It is important to exclude other conditions that may be causing symptoms, including infection (lymphogranuloma venereum, Neisseria gonorrhoeae, herpes simplex virus, syphilis, Giardia duodenalis, amoebiasis), solitary rectal ulcer, psoriatic colitis, chemical colitis and rectal prolapse. ${ }^{178}$

If the diagnosis is correct and standard therapy has failed, then thiopurine therapy should be added, ${ }^{179}$ with escalation to biologics if no response. ${ }^{180-182}$ Many UC trials have excluded proctitis, but a case series of infliximab therapy for proctitis confirms good response. ${ }^{183}$ Patients with refractory proctitis have disabling symptoms but are often systemically well and are usually very reluctant to have proctocolectomy, so many other therapies have been assessed. There are many other treatments based on small trials or case series-for example, rectal tacrolimus $(0.5 \mathrm{mg} / \mathrm{mL}$, dose $3 \mathrm{~mL}$ twice daily)—although active absorption results in significant serum levels and close monitoring and dose adjustment are required to avoid toxicity. ${ }^{184}$

\subsection{Stopping 5-ASA or thiopurine therapy}

Evidence from many UC trials show that patients with a longer duration of remission have lower relapse rates, and duration of remission is an independent predictor, regardless of treatment

Statement 14. We suggest that refractory ulcerative proctitis may require treatment with corticosteroids, immunomodulators and/or biological therapy (GRADE: weak recommendation, very low-quality evidence. Agreement: 95.5\%). 


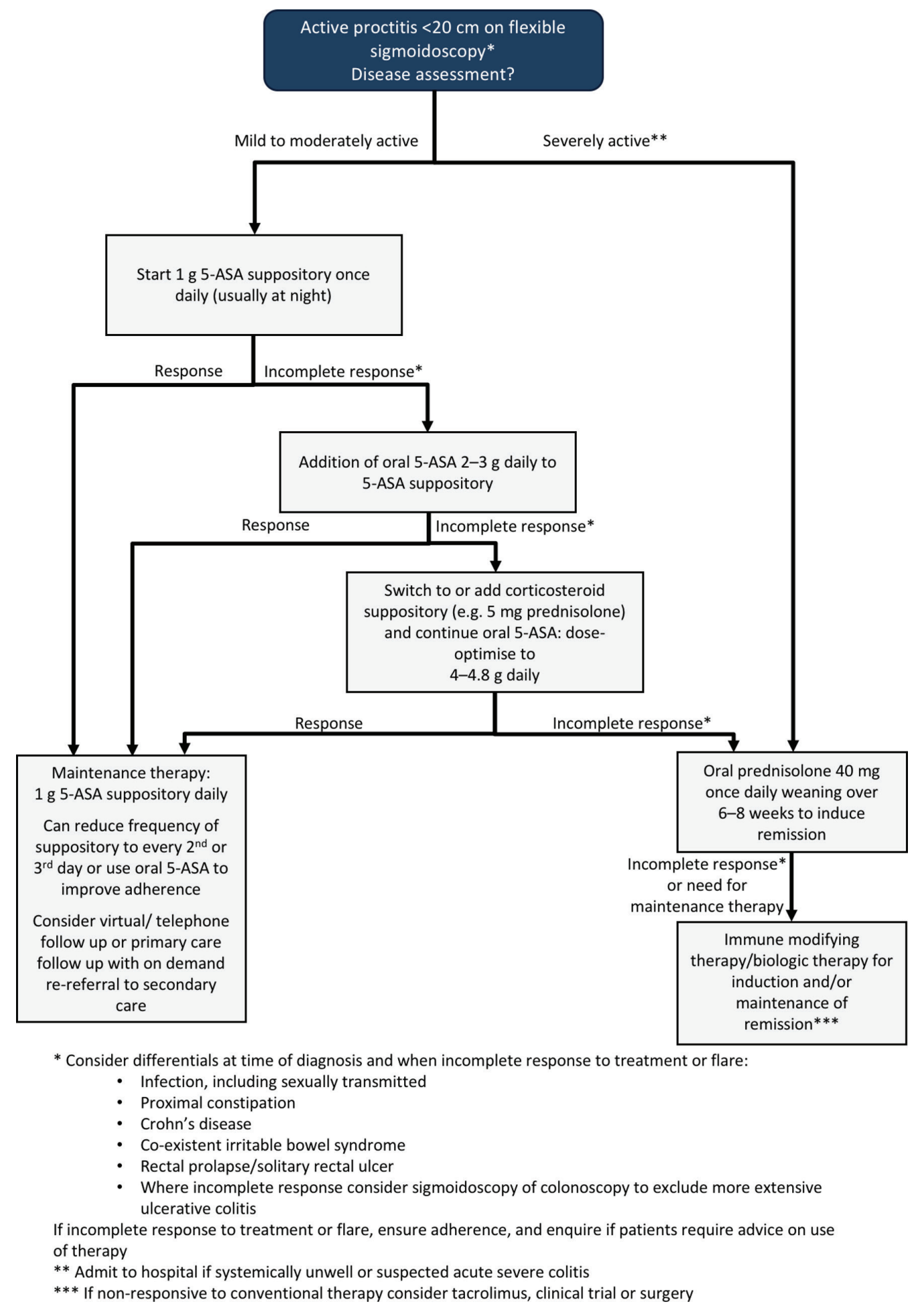

Figure 1 Management of proctitis.

received. ${ }^{185} 186$ Trials have also shown that age is a risk factor with relapse rates inversely proportional to age. ${ }^{185} 187$ A study of mesalazine maintenance therapy evaluated patients with UC in established clinical, endoscopic and histological remission (on the basis of sigmoidoscopy), and divided them into a short remission group (1-2 years) and prolonged remission ( $>2$ years, with a median of 4 years). Patients were randomised to receive mesalazine $1.2 \mathrm{~g}$ daily or placebo. In the short remission group relapse rate at 1 year was $23 \%$ on mesalazine and $49 \%$ on placebo $(p=0.035)$. In the prolonged remission group there was no significant difference whether on mesalazine (relapse in $18 \%)$ or placebo $(26 \%)$. The prolonged remission group were also older and had longer duration of disease. ${ }^{188}$

As well as consideration of the relapse-preventing value of 5-ASA in prolonged remission, the other consideration is the potential colorectal cancer-preventing benefits. There are theoretical reasons why 5-ASA may have colorectal cancer preventive properties, ${ }^{189}$ but unclear whether 5-ASA has benefit independently, or whether the documented benefit relates to mucosal healing. Colorectal cancer risk is related to inflammation. ${ }^{55} 190$ 
Therefore, it is plausible that mucosal healing should be the main goal of colorectal cancer prevention, regardless of the treatment used. ${ }^{191}$ Patients should be advised that 5-ASA is a safe and effective long-term treatment, and should be advised that if they have documented prolonged clinical and endoscopic remission, there is no clear evidence whether continuing 5-ASA adds additional reduction in colorectal cancer risk, but if subsequent assessment after stopping 5-ASA shows mucosal inflammation, 5-ASA should be restarted.

Most of the published data on withdrawal of thiopurines evaluates UC patients in remission for 1-2 years only. In the only randomised controlled withdrawal trial of azathioprine, there was a relapse rate of nearly $60 \%$ at the end of the first year in those who discontinued the drug while in corticosteroid-free clinical remission (for a mean of 11-12 months) compared with $36 \%$ on continued azathioprine. ${ }^{185}$ Other cohort studies with marked heterogeneity in study design and follow-up have reported relapse rates ranging from $21 \%$ to $100 \%$ at 2 years. ${ }^{192}$ A prospective study in patients with UC stopping azathioprine after a minimum of 3 years of therapy in clinical, biological, and endoscopic remission showed a relapse rate of $37 \%$ after a mean follow-up of 55 months. ${ }^{193}$ The risks of relapse are likely to be much lower in patients with prolonged remission (5-10 years) and the benefits of continuing therapy are likely to be less than the risk of drug toxicity, including lymphoma. In the event of relapse there is a very high likelihood of regaining remission; $92 \%$ in one observational study. ${ }^{194}$ Relapse rates are lower in those continuing 5-ASA therapy, 193195 so all patients stopping thiopurines should be receiving 5-ASA if tolerated. In the absence of high-quality data, stopping thiopurines may be an option for those in long term (5-10 years or more) clinical, endoscopic and histological remission, after appropriate patient counselling. Periodic calprotectin monitoring may be a cost-effective method of monitoring ongoing mucosal healing after cessation.

\subsection{Acute severe ulcerative colitis}

Between $15 \%$ and $25 \%$ of patients with UC will require hospitalisation for an acute severe flare of disease at some stage in the natural history of their disease, often as the index presentation. ${ }^{196}$ Acute severe ulcerative colitis (ASUC) is a potentially life-threatening condition, characterised by clinical and laboratory assessment using the modified Truelove and Witts criteria ${ }^{102}$ or, in the case of adolescent patients, the Paediatric UC activity index. ${ }^{197}$ In current practice C-reactive protein is measured far more than ESR, and has the advantage of a less non-specific increase with age. ${ }^{198}$ A retrospective UK study (1950-2007) showed that the colectomy rate during first admission with ASUC was $19 \%$, but after several admissions rose to $38.2 \%{ }^{199}$ In the biologics era, the colectomy rate after admission for ASUC in the CONSTRUCT trial was $23 \%$ during the inpatient stay, ${ }^{200}$ and in another study was $19 \%$ at 2 years. ${ }^{201}$

Statement 15 . We recommend that adult patients with acute severe ulcerative colitis (ASUC) (defined by the modified Truelove and Witts criteria as $>6$ bloody stools per day and systemic toxicity with at least one of: temperature $>37.8^{\circ} \mathrm{C}$, pulse $>90 \mathrm{bpm}$, haemoglobin $<105 \mathrm{~g} / \mathrm{L}$ or C-reactive protein $>30 \mathrm{mg} / \mathrm{L}$ ) or adolescents with a Paediatric Ulcerative Colitis Activity Index (PUCAI) score of 65 or more should be admitted to hospital for assessment and intensive management (GRADE: strong recommendation, low-quality evidence. Agreement: 100\%).
Statement 16 . We recommend that patients presenting with possible acute severe colitis should have urgent inpatient assessment and blood tests (FBC, CRP, U\&E, LFTs and magnesium), stool culture, Clostridium difficile assay, radiological imaging (AXR or $\mathrm{CT}$ ) and flexible sigmoidoscopy (GRADE: strong recommendation, very low-quality evidence. Agreement: 97.9\%).

All patients admitted with ASUC should have baseline bloods (FBC, CRP, U\&E, LFT and magnesium, stool culture and Clostridium difficile assay, radiological imaging (AXR or CT) and flexible sigmoidoscopy, with close monitoring after admission. MRI and ultrasound may have a role in determining the extent of colitis, but are less sensitive than CT in detecting perforation. The results of these tests will also assist in determining the prognosis for that admission (particularly to predict corticosteroid failure and the need for colectomy). ${ }^{196202}$ Early flexible sigmoidoscopy is important to confirm diagnosis and obtain histology including evaluation for cytomegalovirus (CMV) (see Section 5.1.4:cytomegalovirus infection in IBD) and prognosis. Stool culture and microscopy should be performed routinely, as soon as practicable, to exclude pathogenic bacteria including testing for C. difficile toxin. An accurate stool chart, recording frequency, consistency and the presence of blood should be kept, with patients instructed to keep this record if they are able to do so. As nearly half are likely to fail intravenous corticosteroids, ${ }^{200}$ consideration should be given to pre-biologics screening for all ASUC patients on admission.

\subsubsection{Corticosteroid therapy for ASUC}

Intravenous corticosteroids such as hydrocortisone $100 \mathrm{mg}$ four times daily or methylprednisolone $60 \mathrm{mg}$ every 24 hours are the cornerstone of treatment of ASUC. ${ }^{106}{ }^{203-205}$ Methylprednisolone has less mineralocorticoid effect than hydrocortisone at these doses and so causes significantly less hypokalaemia. ${ }^{206}$ There is no real advantage from giving higher doses and a bolus injection is as effective as a continuous infusion. ${ }^{207}$ Although a meta-regression analysis failed to show a relationship between dose and colectomy rate above the equivalent of $60 \mathrm{mg}$ methylprednisolone, ${ }^{106} 400 \mathrm{mg}$ hydrocortisone is equivalent to $80 \mathrm{mg}$ methylprednisolone. In children and adolescents, a dose of $1-1.5 \mathrm{mg} / \mathrm{kg} / \mathrm{day}$ up to a maximum of $60 \mathrm{mg}$ is recommended. ${ }^{208}$

In the landmark study by Truelove and Witts, ${ }^{102}$ oral cortisone $100 \mathrm{mg}$ daily was given to 213 patients with acute flares of varying severity. Clinical remission was achieved in $41 \%$ of steroid treated patients versus $16 \%$ on placebo. In a subsequent study, 49 patients with severe UC were treated with intravenous prednisolone 21-phosphate and a clinical remission rate of $73 \%$ was noted 5 days after treatment. ${ }^{209}$ Definitions and terms differed widely in these studies compared with outcome measures in use today. A systematic review of 32 trials of steroid therapy for ASUC involving 1991 patients reported an overall

Statement 17. We recommend that patients with ASUC should be treated with high-dose intravenous corticosteroids such as methylprednisolone $60 \mathrm{mg}$ daily or hydrocortisone $100 \mathrm{mg}$ 6-hourly (GRADE: strong recommendation, high-quality evidence) and should receive prophylactic low-molecular weight heparin (GRADE: strong recommendation, moderate-quality evidence. Agreement: 97.8\%). 
Good Practice Recommendation 1. Corticosteroid treatment for patients with suspected ASUC should not be delayed pending results of stool cultures and Clostridium difficile assay (Agreement: 95.6\%).

response to steroids of $67 \%$, with $29 \%$ (95\% CI 28\% to $31 \%$ ) having colectomy. Mortality was $1 \%(n=22 / 1991 ; 95 \%$ CI $0.7 \%$ to $1.6 \%$ ) and none of these outcomes changed between 1974 and $2006\left(\mathrm{R}^{2}=0.07, \mathrm{p}=0.8\right) .{ }^{106}$ In the CONSTRUCT cohort, after excluding those with infections or alternative diagnoses, response rate to intravenous steroids was 49\%. ${ }^{200}$ Extending therapy beyond 7-10 days carries no additional benefit and increases toxicity. ${ }^{104} 105$ Patients should be assessed for a clinical and biochemical response after 3 days of intravenous steroid therapy to determine the need for salvage medical or surgical

Risk of venous thromboembolism (VTE) is 2-3 fold higher compared with inpatients without IBD, so prophylactic subcutaneous low molecular weight heparin is important ${ }^{204211212}$ and does not precipitate or exacerbate colonic bleeding. ${ }^{213}$

ASUC patients should have stool culture and Clostridium difficile toxin assay. ${ }^{214} 215$ C. difficile infection has been associated with a worse outcome in hospitalised IBD patients. ${ }^{216-218}$ If C. difficile is detected (or strongly suspected), treatment with corticosteroids should not be withheld, but additional treatment with oral vancomycin given ${ }^{219}$ (see Section 5.1.3: Common Disease Considerations, Clostridium difficile infection associated with IBD). Amoebiasis should be considered in those with recent travel to endemic areas, in whom consideration should be given to addition of metronidazole pending stool microscopy and culture.

\subsubsection{Predictors of outcomes of ASUC}

Most studies of factors predicting colectomy in ASUC look at patients treated with intravenous steroids \pm salvage therapy and do not specifically look at factors after ciclosporin or infliximab have been started. Low albumin is predictive of colectomy, ${ }^{220} 221$ with one study showing that albumin on day 3 of corticosteroid treatment was the only predictor of colectomy on multivariable analysis. $^{222} \mathrm{~A} \mathrm{CRP} /$ albumin ratio of 0.85 on day 3 of intravenous steroids also predicted colectomy with a sensitivity of $70 \%$, specificity $76 \% .^{223}$ Flexible sigmoidoscopy performed with care is safe in ASUC and gives important information about disease severity. ${ }^{224}$ Severe endoscopic lesions (deep ulcers, extensive loss of mucosal layers, well-like ulcers or large erosions) have been associated with non-response to corticosteroids ${ }^{225}$ and colectomy. ${ }^{226} 227$ A study of 89 ASUC patients treated with intravenous corticosteroids and/or infliximab or ciclosporin used the UCEIS and showed that almost all patients with a UCEIS score of 7 or more on admission required rescue therapy, ${ }^{228}$ and another study shows UCEIS is predictive of need for rescue therapy and for surgery. ${ }^{229}$

\subsubsection{Ciclosporin or infliximab rescue therapy for ASUC}

Patients who do not respond to corticosteroid therapy after 3 days should be considered for second-line 'rescue' therapy or surgery. Early clinical risk stratification tools can be used to predict failure of medical therapy (table 5); those at high risk of failure should be offered rescue therapy after discussion with the colorectal surgeon (figure 2). therapy. ${ }^{106210}$

Statement 18. We recommend that patients with ASUC failing to respond by day 3 , as judged by a suitable scoring system, should be treated with rescue therapy in the form of intravenous infliximab or ciclosporin for patients who have not previously failed thiopurine therapy (GRADE: strong recommendation, highquality evidence. Agreement: 97.8\%).

\subsubsection{Ciclosporin in ASUC}

The striking benefits of ciclosporin in ASUC were shown in a 1994 paper. ${ }^{230}$ A randomised controlled trial showed that $2 \mathrm{mg} / \mathrm{kg}$ intravenous ciclosporin was as effective as $4 \mathrm{mg} /$ $\mathrm{kg}$ in ASUC, with response rates at day 8 of $83 \%$ and $82 \%$, respectively with no difference in colectomy rates. ${ }^{231}$ Reviews have shown short-term colectomy rates in patients receiving intravenous ciclosporin vary from 26\% to 47\%. ${ }^{196} 210$ The initial ciclosporin dose is $2 \mathrm{mg} / \mathrm{kg} /$ day intravenously with a target trough ciclosporin concentration of $150-250 \mathrm{ng} / \mathrm{mL}^{231}$ Responders should be converted to an oral dose twice the intravenous dose and administered in divided doses twice daily with a target trough concentration of $100-200 \mathrm{ng} / \mathrm{mL} .{ }^{200}$ Oral ciclosporin should be continued for several months as bridging therapy, ${ }^{230-234}$ and long-term outcomes are much better if thiopurine maintenance therapy is given, rising from $40 \%$ to $66 \%$ in one study. ${ }^{235}$ Those requiring ciclosporin salvage therapy who have already failed thiopurine therapy have much worse outcomes. $^{233236}$

Large case series of ciclosporin therapy in UC have reported a significant toxicity profile, with serious infections in 5\% of patients and mortality in 1-3\%.33 237238 Major adverse events related to ciclosporin therapy include nephrotoxicity $(6.3 \%)$, seizures (3.6\%), anaphylaxis $(0.9 \%)$ and death $(1.8 \%){ }^{238}$

\subsubsection{Infliximab in ASUC}

Infliximab is also effective in ASUC. ${ }^{220} 239240$ Long-term follow-up data of the original Swedish trial ${ }^{239}$ which studied a single infusion of infliximab showed a significantly lower colectomy rate compared with controls $(50 \%$ vs $76 \% ; \mathrm{p}=0.01)$ at 3 years, without use of maintenance infliximab. ${ }^{241}$ Contraindications to use include latent tuberculosis (TB), active sepsis,

Table 5 Indices predictive of failure of corticosteroid therapy for ASUC

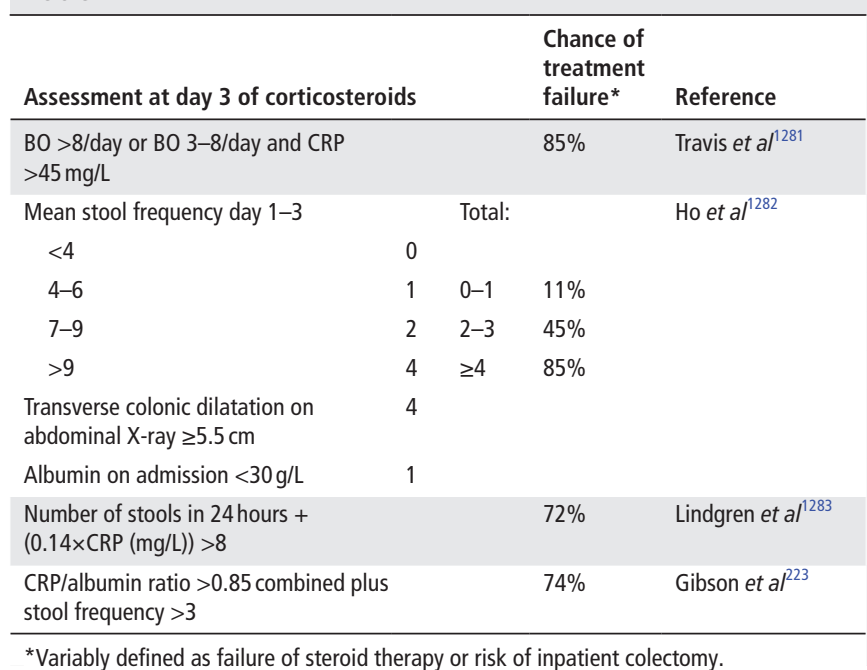




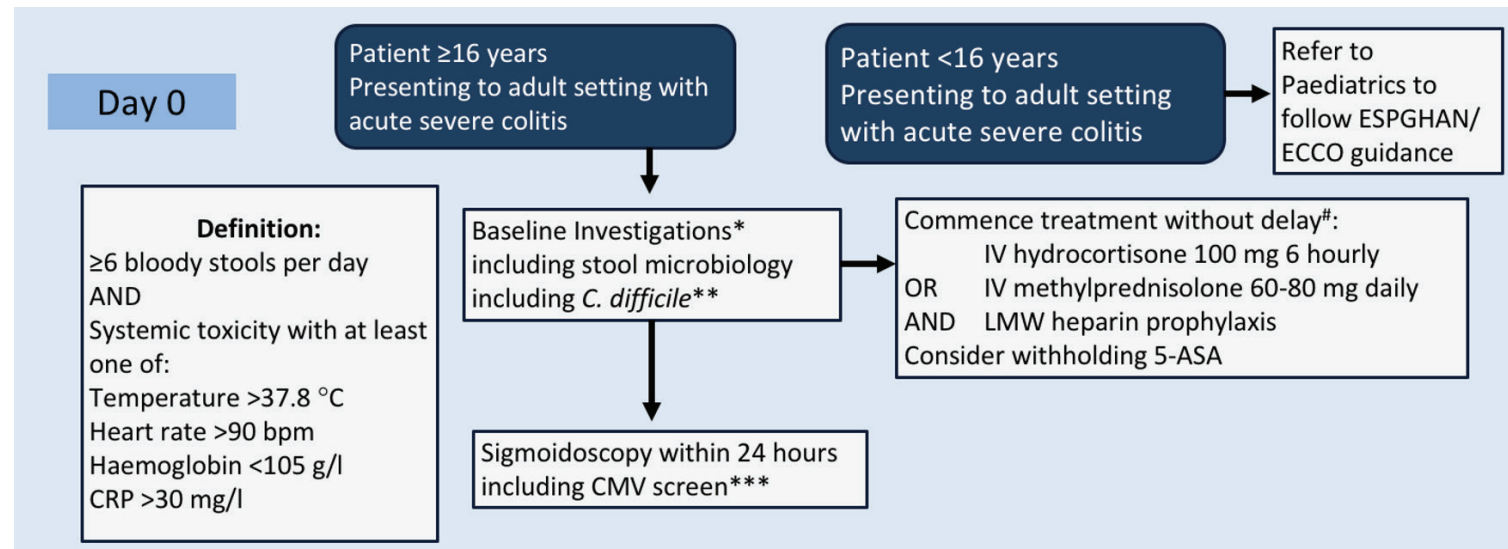

Daily throughout stay: Senior gastroenterology review; FBC, U\&E, CRP, imaging + surgical review if continued systemic toxicity, severe abdominal pain, oedema with low albumin or suspicion of toxic megacolon or perforation. CT preferable to abdominal $\mathrm{X}$-ray if severe complications, notably perforation, are suspected

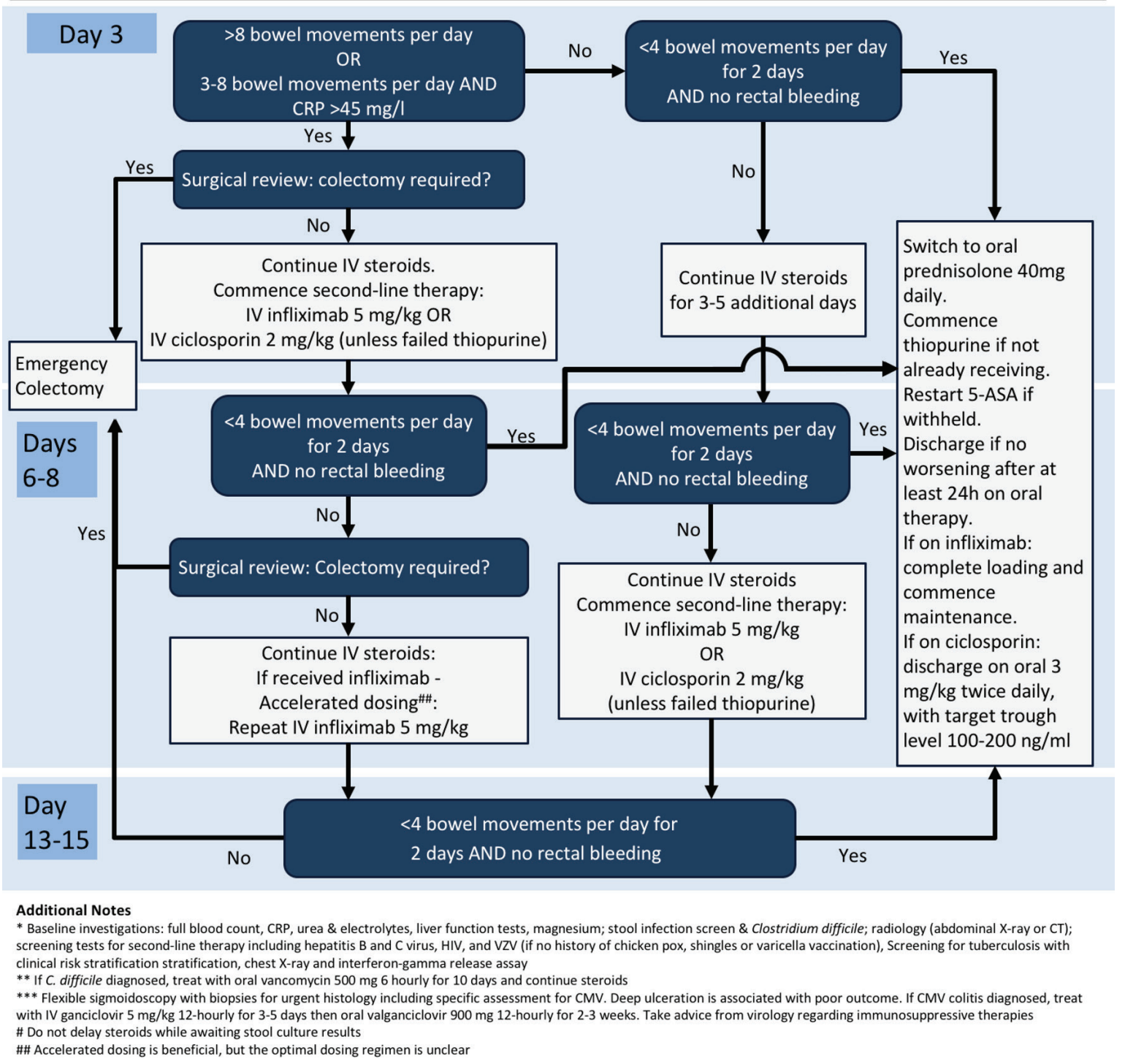

Figure 2 Management of acute severe ulcerative colitis.

congestive heart failure and demyelination, and screening is required before starting treatment. Combination therapy using azathioprine with infliximab has a synergistic effect, with evidence of benefit in raising infliximab levels and reducing antibody formation against infliximab, ${ }^{242}$ even in patients who have previously failed monotherapy with thiopurines. Azathioprine or mercaptopurine should be started during hospitalisation and continued after discharge. Once patients have responded well to rescue therapy, corticosteroid doses should be tailed. Prophylaxis for Pneumocystis jirovecii should be given 
for those on $20 \mathrm{mg}$ prednisolone or more (see Section 5.2.8.6: Common Disease Considerations, Prevention of Pneumocystis jirovecii infection).

\subsubsection{Comparison of infliximab and ciclosporin in ASUC}

Head-to-head comparisons between ciclosporin and infliximab have demonstrated equivalent efficacy. In the open label CySIF trial, 115 patients previously naïve to infliximab and ciclosporin, who had a Lichtiger score $>10$ points (range $0-21$ ) and colitis refractory to at least 5 days of intravenous steroids, were randomised in a 1:1 ratio to receive intravenous ciclosporin $(2 \mathrm{mg} / \mathrm{kg}$ per day for 1 week followed by oral drug until day 98) or infliximab $(5 \mathrm{mg} / \mathrm{kg}$ on days 0,14 and 42$) .{ }^{243}$ In both groups, azathioprine was started at day 7 in patients with a clinical response. The primary end-point was treatment failure defined by absence of a clinical response at day 7 , a relapse between day 7 and day 98, absence of steroid-free remission at day 98, a severe adverse event leading to treatment interruption, colectomy or death. There was no statistically significant difference between treatment failure in patients given ciclosporin (60\%) and infliximab (54\%). Nine (16\%) patients in the ciclosporin group and 14 $(25 \%)$ in the infliximab group had severe adverse events, which was also not statistically different. Similar mucosal healing rates (47\% ciclosporin, 45\% infliximab) and colectomy rates $(17 \%$ ciclosporin, 21\% infliximab) were achieved in both groups. There was no difference in colectomy-free survival at 1 and 5 years in patients treated with either ciclosporin or infliximab. ${ }^{244}$

The CONSTRUCT trial was an open-label pragmatic randomised trial in 270 patients, who were randomly allocated $(1: 1)$ to receive either infliximab $(5 \mathrm{mg} / \mathrm{kg}$ intravenous infusion given over 2 hours at baseline and again at 2 weeks and 6 weeks after the first infusion) or ciclosporin $(2 \mathrm{mg} / \mathrm{kg}$ per day by continuous infusion for up to 7 days followed by twice-daily tablets delivering $5.5 \mathrm{mg} / \mathrm{kg}$ per day for 12 weeks). The primary outcome was quality-adjusted survival; the area under the curve of scores from the Crohn's and Ulcerative Colitis Questionnaire completed by participants at baseline, 3 months, and 6 months, then every 6 months from 1 year to 3 years. ${ }^{200}$ There was no statistically significant difference between the two groups for the primary end-point as well as the secondary end-point of colectomy rates, time to colectomy, serious adverse events or death. Colectomy rates were $29 \%$ for infliximab and $30 \%$ for ciclosporin at 3 months, and $35 \%$ and $45 \%$ respectively at 1 year, with no significant difference between the treatments. ${ }^{200}$ However, treatment with infliximab was associated with greater cost of treatment compared with ciclosporin.

A meta-analysis of infliximab and ciclosporin randomised controlled trials shows no difference in response up to 1 year. ${ }^{245}$ In the Spanish ENEIDA registry, where patients with steroid unresponsive ASUC were treated with ciclosporin (the majority of whom were given azathioprine maintenance therapy), the colectomy rate at 5 years was $25.4 \%$. For those treated with infliximab, either continuing repeat infusions, combination therapy with azathioprine, or azathioprine only, the 5-year colectomy rate was similar at $26.2 \% .^{234}$

Mortality from infliximab trials is comparable to data on ciclosporin (0-2\%). ${ }^{196210234}$ The most significant risk for both infliximab and ciclosporin relates to those on either of these drugs combined with high-dose corticosteroids who fail medical treatment and go on to colectomy with deteriorating physical condition (anaemia, hypoalbuminaemia and oedema) where surgical complications are a significant concern (see Section 3.12.9: Ulcerative Colitis, Surgery for acute severe UC).
Statement 19. We suggest that patients treated with infliximab for ASUC who have not responded sufficiently to a $5 \mathrm{mg} / \mathrm{kg}$ dose 3-5 days after first infusion should be treated with an accelerated induction regimen after colorectal surgical review to determine whether emergency colectomy is required (GRADE: weak recommendation, low-quality evidence. Agreement: $95.7 \%)$.

Ciclosporin and infliximab are equally effective in ASUC. Infliximab, however, is simpler to use and is thus used much more widely now. Although infliximab was more expensive, use of biosimilars is bringing costs down.

\subsubsection{Sequential therapy (infliximab and ciclosporin) in refractory} ASUC

Sequential therapy with infliximab and ciclosporin can be associated with profound immunosuppression and potentially risks serious adverse events and infections and is not recommended, ${ }^{246} 247$ although a recent review suggested that the risks were not excessive (on the basis of low-quality data). ${ }^{248}$ In the ENEIDA registry sequential therapy was defined as treatment with the second agent (ciclosporin or infliximab) within 3 months after the steroid-refractory ASUC attack, and was used in $10 \%$ of those with long-term outcomes available. ${ }^{234}$ The median time between treatments was 24 days (95\% CI 2.5 to 75.0 ), and showed comparable safety to use of one rescue therapy only. The greatest risk of this approach is delaying surgery in a patient whose physical condition is deteriorating.

\subsubsection{Accelerated infliximab induction regimen in ASUC}

Recent studies have demonstrated an association between higher serum levels of anti-TNF and better outcomes. ${ }^{249}$ A post hoc analysis of ACT 1 and 2 clinical trials noted that patients in the lowest quartile of infliximab serum concentration were less likely to achieve clinical response, remission and mucosal healing, independent of randomised dose $(5 \mathrm{mg} / \mathrm{kg}$ or $10 \mathrm{mg} / \mathrm{kg}){ }^{250}$

Various factors, including a high TNF burden in ASUC, proteolytic degradation of anti-TNF associated with increased drug clearance and faecal losses from increased gut permeability due to severe inflammation, support the need for dose optimisation of infliximab in the acute phase. ${ }^{251}$ In a recent study, serum and non-inflamed tissue anti-TNF drug levels were shown to be related, but serum and tissue drug levels were not associated in inflamed tissue. ${ }^{252}$ This suggests that high mucosal cytokine levels during inflammation act as a 'sink' for drug, and thus a higher serum level of the drug may be required to neutralise tissue TNF. In another study, a high baseline CRP $(>50 \mathrm{mg} / \mathrm{L})$ and a low serum albumin $(<35 \mathrm{~g} / \mathrm{L})$, as surrogates for severe inflammation and extensive colitis, independently correlated with lower infliximab concentrations from week $0-6 .{ }^{253}$

There is further evidence of benefit with accelerated infliximab dosing in ASUC. Three doses of accelerated infliximab dosing at $5 \mathrm{mg} / \mathrm{kg}$, administered over a median 24 days to steroid-refractory patients, demonstrated a colectomy rate of $6.7 \%$ compared with $40 \%$ in a retrospective cohort (standard $5 \mathrm{mg} / \mathrm{kg}$ induction at 0 , 2 and 6 weeks). ${ }^{254}$ For those completing induction therapy, the colectomy rates during follow-up were similar (median follow-up of 2.4 years for standard dosing and 1.6 years for accelerated dosing regimen). A recent review suggested that dose intensification may benefit half of patients with ASUC treated with infliximab, with case control studies showing that 1-2 extra infusions in the first 3 weeks can dramatically reduce colectomy rates. ${ }^{251}$ 
Good Practice Recommendation 2. In acute severe ulcerative colitis, delay in surgery is associated with an increased risk of surgical complications, mandating early referral and direct involvement of specialist colorectal surgical and stoma care teams (Agreement: 100\%).

Patients treated with infliximab who are not responding sufficiently to a $5 \mathrm{mg} / \mathrm{kg}$ dose after 3-5 days can be treated with an early repeat infusion, particularly in those with a low albumin (below $35 \mathrm{~g} / \mathrm{L}$ ). Some clinicians use an initial $10 \mathrm{mg} / \mathrm{kg}$ dose as salvage therapy but there is as yet insufficient data to demonstrate the value of this in comparison to a $5 \mathrm{mg} / \mathrm{kg}$ dose. ${ }^{255}$ Optimal timing and dose $(5 \mathrm{mg} / \mathrm{kg}$ or $10 \mathrm{mg} / \mathrm{kg}$ ) are as yet unclear. Accelerated dosing should only be given after colorectal surgical review, with agreement that colectomy is not required imminently (see figure 2). Further studies are required to evaluate the use of drug levels and biomarkers to determine personalised dosing for patients.

\subsubsection{Colectomy for ASUC}

Outcomes from ASUC were revolutionised by the use of corticosteroids and the introduction of timely surgery in those who had not responded. Mortality plummeted from 24\% to $<1 \%$ in the landmark studies. ${ }^{102256}$ Despite rescue therapy, a significant number of patients still go to surgery in the acute situation. Although meta-analysis has not shown evidence that infliximab increases postoperative complications in $\mathrm{UC},{ }^{257}$ a recent series has shown that outcomes for surgery have been worse since the introduction of biologics. ${ }^{258}$ One possible explanation for this discrepancy would be that, in the case of ASUC, using biologics as rescue therapy after failure of corticosteroids creates a delay in surgery. Although thromboembolic complications are a significant risk, ${ }^{259}$ a recent study did not show a difference between those going to surgery after steroids only and those receiving steroids and 'rescue therapy' prior to colectomy. Both groups however had been inpatients for a long time before surgery (10 vs 12 days). ${ }^{260}$

Timely decision-making is therefore important in order to prevent delays or prolongation of medical therapy, as those failing medical therapy and undergoing colectomy have higher postoperative complication rates after delayed surgery. ${ }^{105}$ Prolonged admission prior to surgery was the only significant predictor of postoperative complications in another study. ${ }^{261}$ In a study from the USA, mortality in those who had colectomy within 3 days of admission was used as a comparator. ${ }^{262}$ For those with colectomy up to 6 days, the adjusted ORs for postoperative death was 2.12 (95\% CI 1.13 to 3.97$)$ and 2.89 (95\% CI 1.41 to 5.91 ) for colectomy up to 11 days.

Multidisciplinary team involvement with gastroenterologist, colorectal surgeon, and stoma therapist enables better management. ${ }^{196263}$ Surgical input at an early stage helps patients to understand that colectomy is an important treatment option and is not an outcome to be avoided at any cost. Prompt joint decision-making is essential to avoid unnecessary delays (figure 2).

Statement 20. We recommend that patients with ASUC who have not responded within 7 days of rescue therapy with infliximab or ciclosporin, or those with a deterioration or complications before that time (including toxic megacolon, severe haemorrhage or perforation) require subtotal colectomy and ileostomy, with preservation of the rectum (GRADE: strong recommendation, very low-quality evidence. Agreement: 97.4\%).

\subsection{Surgical management in UC}

\subsubsection{Emergency colectomy for UC}

Surgery in ASUC is indicated when the disease is medically resistant, there are intolerable side effects of medication, or when there is life-threatening haemorrhage, toxic megacolon or perforation. ${ }^{264}$ Toxic megacolon is a devastating complication of severe colitis, characterised by radiographic distension of the colon often with fever, tachycardia, neutrophil leucocytosis and anaemia.

In this setting, the operation of choice is a subtotal colectomy and end ileostomy with long rectal stump. ${ }^{259} 265266$ This could be performed laparoscopically or open according to local expertise, although a laparoscopic approach is likely to result in shorter length of stay and reduced risk of infectious complications. $^{267-269}$

Care should be taken to avoid leakage from the rectosigmoid stump, either by formation of mucous fistula, locating the top of the remnant in the subcutaneous tissue of the abdominal wall, or closing the stump but leaving a transanal catheter in the initial postoperative period to prevent stump 'blow-out', with no clear evidence of the preferred technique. ${ }^{269}$ If the patient wishes to consider restoration of intestinal continuity with a proctectomy and ileoanal pouch, this should not be undertaken in the acute setting, given the significant risk of complications in a patient who is likely to be clinically unwell, hypoalbuminaemic and on immunosuppressive medication. Timing of pouch surgery should be an individualised decision with multidisciplinary input, with a minimum of 3 months and preferably 6 months from the initial subtotal colectomy in order that adhesions may be safely manageable and the patient allowed time to generally recover from the initial procedure. A population cohort study from Sweden showed that the median time to subsequent reconstructive surgery is almost 12 months. ${ }^{270}$ At the time of ileoanal pouch surgery, a temporary covering loop ileostomy will reduce the clinical anastomotic leak rate as well as the septic sequelae of a leak, but may be omitted in optimal circumstances. ${ }^{269}$ A subsequent anastomotic leak from a non-defunctioned ileal pouchanal anastomosis would generally require defunctioning.

\subsubsection{Outcomes after colectomy for UC}

Proctocolectomy followed by ileal pouch anal anastomosis (IPAA) is well-established as a management option for UC. Multiple case series have demonstrated good outcomes in terms of quality of life, with a majority of patients indicating they would undertake the same procedure again..$^{271-274}$ In one of the larger series, 95\% of patients reported good or excellent functional and quality of life outcomes over up to 10 years of follow-up. ${ }^{273}$ A further study showed no deterioration in functional outcome over 10 years or more. ${ }^{275}$ Comparison with medical therapies are difficult since there are no published comparative data, and in the absence of randomisation there would be selection bias. Comparison of IPAA to end ileostomy suggests that both are associated with equivalent quality of life, and the decision should be a matter of patient choice after detailed explanation and counselling.

Statement 21. We suggest that surgical resection of the colon and rectum in ulcerative colitis should be offered to patients who have chronic active symptoms despite optimal medical therapy. lleoanal pouch reconstruction or end ileostomy provide equivalently good quality of life, and are a matter of patient choice (GRADE: weak recommendation, very low-quality evidence. Agreement: 94.4\%). 
Statement 22. We suggest that pouch surgery should be performed in specialist high-volume referral centres (GRADE: weak recommendation, low-quality evidence. Agreement $97.4 \%)$.

Functional outcomes are just as good after laparoscopic-assisted pouch surgery. ${ }^{276}$

Ileoanal pouch surgery is not currently a centralised service in the UK. However, data from the UK suggest that high-volume centres (more than 100 procedures between 1996 and 2008) had lower pouch failure rates. ${ }^{277}$ In this study, high-volume centres had a pouch failure rate of $5.2 \%$, similar to the rate of $5.3 \%$ reported from the Cleveland Clinic (5.3\%) in the USA. ${ }^{273}$ A further study showed that there is a significant learning curve for pouch surgery, with improving outcomes dependent on the number of procedures undertaken for both trainees and senior staff. $^{278}$ Likewise, there is evidence that management of leaks after IPAA procedure improves with increasing experience, and results in better pouch salvage rates. ${ }^{279}$

\subsubsection{Surgery in UC patients with primary sclerosing cholangitis}

While the occurrence of complications following IPAA surgery in patients with co-existent UC and primary sclerosing cholangitis (PSC) is high, the risk of pouch failure is low. The most common short- and long-term complication is pouchitis, with acute, recurrent acute and chronic pouchitis all occurring frequently. The largest published series of 100 patients with co-existent UC and PSC with IPAA followed for a median of 5.9 years, reports 30 -day complication rates of $39 \%$ and overall pouchitis rates of $64 \% .{ }^{280}$ Pouch excision was only necessary in $3 \%$ of patients in this series. Other smaller series present similar data with high rates of pouchitis, although whether the rate is higher than in the non-PSC UC cohort is unclear, with data conflicting. ${ }^{281-283}$ For PSC patients with UC undergoing liver transplantation, graft outcomes are better for those who have an end ileostomy after colectomy compared with colectomy and IPAA. ${ }^{284}$ Graft loss was mainly associated with hepatic artery thrombosis and biliary strictures. The risk associated with IPAA is not dependent on the timing of colectomy in relation to the liver transplantation. Patients can be offered pouch formation if they have PSC as long as they understand the potential implications.

\subsubsection{Fertility and delivery after restorative proctocolectomy}

Any pelvic surgery incurs a risk to fertility. Whereas there is evidence that medical treatment of UC does not impair female fertility, ${ }^{285}$ a retrospective systematic review and meta-analysis of fertility rates 1 year after open IPAA surgery showed 63\% infertility with a relative risk of 3.91 associated with the surgery, ${ }^{286}$ corroborating an earlier meta-analysis showing an infertility rate of $15 \%$ for medically-treated UC and $48 \%$ after IPAA. ${ }^{287}$ Time to conception is longer post-surgery, with a more frequent necessity of in vitro fertilisation and higher incidence of caesarean section. ${ }^{288}$ It is likely that Fallopian tube scarring in relation to the procedure is the cause of infertility. ${ }^{286}$ In selected female

\section{Good Practice Recommendation 3. Patients undergoing} colectomy who have co-existent ulcerative colitis and primary sclerosing cholangitis should be advised that there is an increased risk of pouchitis, to inform decision-making regarding ileoanal pouch formation or permanent ileostomy (Agreement: 100\%).
Good Practice Recommendation 4. For females requiring emergency subtotal colectomy and ileostomy, decisions regarding proctectomy and ileoanal pouch reconstruction should be discussed because of the potential for impaired fertility. Decisions should be personalised, including use of laparoscopic techniques and the option of delaying until after completion of the family (Agreement: 100\%).

patients without proctitis, subtotal colectomy with ileorectal anastomosis (IRA) may be preferable as this does not impair fertility, ${ }^{289}$ although this procedure is not suitable for all and the long-term risk of rectal malignancy remains following IRA. There is evidence that pelvic scarring is much reduced when IPAA is performed laparoscopically. ${ }^{290}$ Consistent with this, time to first spontaneous pregnancy following IPAA is shorter in those undergoing laparoscopic surgery in comparison to open procedures. ${ }^{291}$ In a retrospective questionnaire-based study comparing fertility in 15 women following laparoscopic IPAA to that in a control group having appendectomy, infertility was demonstrated in $27 \%$ which was no different to the control group, ${ }^{292}$ suggesting that laparoscopic restorative proctocolectomy may be the preferred option for female patients of reproductive age.

\subsection{Pouches and pouchitis}

\subsubsection{Assessment of new symptoms after IPAA}

Up to $50 \%$ of patients will develop pouchitis at some time after IPAA (as many as $40 \%$ in the first year). Typical symptoms of pouchitis include increased bowel frequency, urgency, nocturnal seepage or incontinence, pelvic discomfort and abdominal cramps (table 6). ${ }^{293}$ Diagnosis is based on the presence of symptoms in conjunction with endoscopic appearance and histology to rule out other conditions. Patients who develop symptoms may not have pouchitis: post-surgical complications should be considered particularly within the first year after surgery. ${ }^{294}$ This can be due to fistula development, pelvic sepsis and anatomical problems related to pouch construction (for instance, long cuffs with flutter valve effects or mechanical causes of poor pouch emptying). A study in 68 patients with antibiotic-dependent or refractory primary idiopathic pouchitis, who had had both pouchoscopy and pelvic MRI, showed that $38 \%$ had chronic peri-pouch sepsis. Those with sepsis more often had inflammation in the upper pouch only, and although some had a collection that could be drained radiologically, outcomes were poor with most remaining on long-term antibiotics and nearly a third having a defunctioning stoma or pouch excision. ${ }^{293}$ In patients with chronic pouchitis $20 \%-30 \%$ have secondary causes for their symptoms, which include CMV infection, Candida, Clostridium difficile, ${ }^{295}$ ischaemia, autoimmune disorder, or damage from radiotherapy, chemotherapy or non-steroidal anti-inflammatory drugs. ${ }^{180296}$ A proportion may have Crohn's disease, with reported frequency ranging from $3.6 \%-13 \% .^{297}$

Endoscopy is the main tool in the diagnosis and differential diagnosis of pouchitis. The most commonly used clinical scores

Good Practice Recommendation 5. Patients with ongoing symptoms after pouch surgery should have pelvic MRI scan, stool culture and Clostridium difficile assay. Pouchoscopy should be performed to assess the pouch, the pre-pouch ileum and the mucosa at the anal transition zone (Agreement: 100\%). 


\begin{tabular}{|c|c|}
\hline Variable & Score \\
\hline \multicolumn{2}{|l|}{ Clinical } \\
\hline Stool frequency (daily) & $\begin{array}{l}0-2(0=\text { usual post-operative frequency, } 1=1-2 \text { stools more than post-operative frequency, } 2=>3 \text { stools more than post }- \\
\text { operative frequency) }\end{array}$ \\
\hline Rectal bleeding & $0-1$ (0=absent/rare, $1=$ present daily) \\
\hline Urgency/abdominal cramps & $0-2$ ( $0=$ absent, $1=$ occasional, $2=$ usual) \\
\hline Fever $\left(>37.8^{\circ} \mathrm{C} / 100.5^{\circ} \mathrm{F}\right)$ & $0-1$ (0=absent, $1=$ present $)$ \\
\hline Endoscopic inflammation & One point for each of: oedema, granularity, friability, loss of vascular pattern, mucous exudate, ulceration) \\
\hline \multicolumn{2}{|l|}{ Histology } \\
\hline Polymorphonuclear leucocyte infiltration & $1-3(1=$ mild, $2=$ moderate + crypt abscesses, $3=$ severe + crypt abscesses $)$ \\
\hline Mean ulceration per low power field & $1-3(1=<25 \%, 2=25-50 \%, 3=>50 \%$ ulceration $)$ \\
\hline
\end{tabular}

Pouchitis, total score $\geq 7$ points; Remission, score $\leq 2$ with endoscopic subscore $\leq 1$.

for pouchitis evaluation are the Pouchitis Disease Activity Index (based on symptoms, endoscopy and histology (table 6) and the simpler Modified Pouchitis Disease Activity Index (symptoms and endoscopy only). ${ }^{298} 299$ In both, endoscopy is key. Pouchoscopy may be best carried out using a gastroscope in view of the flexibility and smaller calibre of this compared with a colonoscope, and a digital examination adds valuable information. At the time of pouchoscopy it is important to assess the pre-pouch ileum as well as the pouch itself, the rectal cuff and anal transition zone (to look for cuffitis). Morphological changes of oedema, loss of vascular pattern, erythema, haemorrhage, erosions/ulceration should be looked for. Ulcers in the afferent loop may indicate Crohn's disease or use of NSAIDs. Inflammation in one half of the pouch with sharp demarcation along the suture line may suggest ischaemia. Staple-line ulcers with normal pouch mucosa elsewhere are common and do not suggest pouchitis. ${ }^{296300}$ Other investigations include pelvic MRI to rule out collections, fistulae or anatomical abnormalities; stool cultures and C. difficile toxin and celiac serology.

\subsubsection{Treatment of acute pouchitis}

Antibiotic therapy is the standard treatment for acute pouchitis, ${ }^{301}$ with observational data demonstrating $96 \%$ of patients have a symptomatic response to antibiotics. ${ }^{302}$ There is very little evidence comparing different antibiotics. However, one small study of 16 patients demonstrated that ciprofloxacin was superior to metronidazole in the treatment of acute pouchitis. ${ }^{303}$ Ciprofloxacin is better tolerated with fewer adverse effects than metronidazole and may therefore be considered first line treatment for acute pouchitis. ${ }^{304}$

There is one trial of probiotics in primary prevention of pouchitis. Forty patients were randomised to a specific probiotic mixture containing eight bacterial strains, or placebo. ${ }^{305}$ Ten percent of the probiotic treated arm developed pouchitis in a year compared with $40 \%$ of the placebo-treated group. An uncontrolled retrospective study reported primary prophylaxis with sulphasalazine $2 \mathrm{~g}$. At a median 68 months, pouchitis occurred in $15 \%$ on sulphasalazine, compared with $64.5 \%$ not given the drug. ${ }^{306}$

Statement 23. We recommend that a 2 week course of ciprofloxacin or metronidazole is the first-line treatment of acute pouchitis (GRADE: strong recommendation, low-quality evidence). We suggest that ciprofloxacin is better tolerated and may be more effective than metronidazole (GRADE: weak recommendation, low-quality evidence. Agreement: 97.2\%).
There are more studies examining secondary prevention of pouchitis. Two trials show benefit for a probiotic mixture of eight bacterial strains given after a 1 month course of antibiotics for patients with chronic relapsing pouchitis. ${ }^{307} 308$ An openlabel study of a probiotic mixture in 28 patients without symptoms, (36\% had previous pouchitis but none had symptoms at study entry) designed to evaluate immunological changes in the pouch, showed progressive improvement in PDAI scores over 1 year, and expansion of mucosal regulatory $\mathrm{T}$ cells, compared with patients on no treatment. ${ }^{309}$ A small study of Lactobacillus rhamnosus GG over 3 months (in patients with previous pouchitis, and with endoscopic pouch inflammation, but no symptoms) showed no benefit. ${ }^{310}$ Overall trial quality in these studies was low, and further trials would be helpful to clarify management. ${ }^{304} 311$ Probiotic therapy can be recommended for prevention of relapsing pouchitis, but there is insufficient evidence to recommend primary prophylactic probiotics after pouch surgery, or even whether this should be given to those at higher risk of pouchitis, such as those with pre-operative extra-intestinal manifestations, primary sclerosing cholangitis or high titres of p-ANCA. ${ }^{312} 313$ Recent studies have shown that alterations in pre-colectomy bacterial microbiota are predictive of subsequent pouchitis, with increases in Ruminococcus gnavus, Bacteroides vulgatus, Clostridium perfringens, and absence of Lachnospiraceae genera (Blautia and Roseburia) and this may inform future treatment strategies. ${ }^{314}$ A small open-label study of faecal microbial transplantation (FMT), (single treatment via nasogastric tube) in 10 patients with chronic pouchitis did not show obvious clinical benefit. ${ }^{315}$

FMT can be used to treat Clostridium difficile infection in the context of ulcerative colitis when first line antibiotics are ineffective. ${ }^{316}$ There is currently no place for FMT in the management of IBD unless complicated by $C$. difficile infection outside of the clinical trial setting.

Anti-diarrhoeal drugs are often used by patients with IPAA to reduce bowel frequency. There is no evidence of benefit in pouchitis treatment although they are often continued. More evidence is required to support this common practice.

\subsubsection{Treatment of chronic pouchitis}

Chronic pouchitis or chronic refractory pouchitis is defined as occurring when patients with pouchitis remain symptomatic despite 4 weeks' treatment with antibiotics. Patients who fail to respond to 2 weeks of an antibiotic, or relapse rapidly after stopping, can be treated with a combination of antibiotics for 4 weeks. Ciprofloxacin plus rifaximin and ciprofloxacin plus tinidazole have been shown to be effective in randomised 
Statement 24. We suggest that chronic pouchitis may be treated with a combination of antibiotics (ciprofloxacin, metronidazole, tinidazole, rifaximin), oral budesonide or oral beclomethasone (GRADE: weak recommendation, very low-quality evidence. Agreement: 85.7\%).
Good Practice Recommendation 6. Ileoanal pouch patients should have long-term specialist follow-up where there is sufficient expertise to deal with complications, and collect longterm outcome data. Rapid access for diagnosis and treatment of suspected pouchitis should be available (Agreement: 100\%). controlled trials. ${ }^{301}$ Oral budesonide $9 \mathrm{mg}$ and oral beclomethasone dipropionate $10 \mathrm{mg}$ daily given for 8 weeks have also been shown to be effective, ${ }^{317} 318$ and if symptoms worsen on stopping, biologics should be considered rather than continuing long-term steroids. Some antibiotic-dependent patients may require long-term rotating antibiotic therapy, and coliform sensitivity testing may help to choose appropriate agents. ${ }^{319}$ Patients with pre-pouch ileitis (occurring in 6\% of patients with pouchitis) represent a high-risk group for chronic refractory pouchitis and should be considered for early escalation to immunomodulatory therapy. ${ }^{320}$

\subsubsection{Treatment with biologics in chronic refractory pouchitis}

Patients developing chronic treatment-refractory pouchitis should be re-evaluated to exclude other causes (see above). A recent systematic review with meta-analysis of the literature regarding the use of anti-TNF therapy for patients with chronic refractory pouchitis has shown moderate to good benefit in eight case series for infliximab and adalimumab therapy with a pooled remission rate of 53\%. ${ }^{301}$ A preliminary report of a small double-blind randomised trial of adalimumab for chronic refractory pouchitis showed that six patients on adalimumab all improved, with only two of seven on placebo improving. The study was too small for statistical significance. ${ }^{321}$ A series of 20 patients with chronic antibiotic-dependent, or refractory pouchitis showed benefit with vedolizumab therapy. Assessed at 14 weeks, PDAI and Oresland scores improved with 17/19 patients able to stop antibiotic therapy. ${ }^{322}$ A small open-label study of 10 patients using tacrolimus enemas $(0.08 \mathrm{mg} / \mathrm{kg})$ showed response in nine, and remission in seven. ${ }^{323}$

\subsubsection{Follow-up of pouch patients}

Ulcerative colitis patients who have undergone ileoanal pouch anastomosis represent a small group of patients, who have undergone a specialised procedure with a 5\%-10\% chance of long-term failure and significant risk of complications, ${ }^{324}$ of which pouchitis is the most frequent. A pouch clinic enhances patient satisfaction, ${ }^{325}$ and facilitates data collection for audit and research purposes. Participation in a national pouch registry is invaluable for this purpose. ${ }^{326}$

\subsubsection{Pouch surveillance}

The risk of neoplasia in pouches is extremely small, with a risk of cancer less than $0.02 \%$ at 20 years. ${ }^{327} 328$ Cancer may arise following pouch surgery within the rectal cuff, within the pouch itself or arise from the skin (squamous cell carcinoma). ${ }^{329}$ Risk of pouch-associated neoplasia is elevated in patients

Statement 25. Chronic refractory pouchitis not responding to antibiotics or locally-acting corticosteroids should be reassessed to consider other factors, and if excluded, we suggest that patients may be offered biologics (GRADE: weak recommendation, low-quality evidence. Agreement: 90.9\%). with a diagnosis of IBD-associated cancer, ${ }^{330}$ family history of colorectal cancer, type $\mathrm{C}$ ileal changes (severe pouchitis rapidly after pouch formation, with moderate to severe villous atrophy), long retained rectal cuff, and primary sclerosing cholangitis. ${ }^{331}$ Although controversial, annual pouchoscopy is recommended by most commentators for these high-risk patient groups. ${ }^{332} 333$ The indications for pouch surveillance in the asymptomatic population without the above risk factors is uncertain, ${ }^{328}$ although abnormal endoscopic findings are not uncommon. ${ }^{334335}$ A decision in this low-risk group to either perform no surveillance unless symptomatic, or 5-yearly, should be discussed with the patient, and documented. Examinations should be performed by an experienced endoscopist.

\section{CROHN'S DISEASE}

\subsection{Diagnosis, classification and assessment}

Crohn's disease is a complex chronic inflammatory gastrointestinal condition with variable age of onset, disease location and behaviour. There is no single unifying definition of Crohn's disease and a combination of investigative modalities is often needed to confirm the diagnosis. The most widely accepted framework for making a diagnosis dates back nearly 30 years. ${ }^{336}$ Factors include an appropriate clinical history and examination, ileocolonoscopy, small bowel imaging, blood tests and histology. Mucosal biopsies from endoscopic procedures or surgical resection specimens show focal or patchy (rather than diffuse) inflammation and/or crypt distortion. Discontinuous segments of disease ('skip lesions'), ileal involvement and granulomatous inflammation are more suggestive of Crohn's disease, as is a tendency for inflammation to be worse in the proximal colon. ${ }^{14}$

Distinguishing between Crohn's disease and UC can sometimes be complicated; partially-treated UC can demonstrate patches of inflammation, backwash ileitis occurs in UC, and granulomas only occur in about half of Crohn's disease patients. Cryptolytic granulomas can occur in UC, diverticular inflammation and all forms of colitis and are very non-specific. Pathologists find it easier to distinguish between IBD and non-IBD than they do to separate Crohn's from UC, and the diagnosis is helped greatly by discussion at a multidisciplinary team meeting with clinicians and pathologist present. ${ }^{14}$ Studies have shown about $3 \%$ of UC patients will be reclassified as Crohn's colitis, and conversely a small number $(0.6-3 \%)$ will be reclassified to UC after an initial diagnosis of Crohn's disease. ${ }^{19} 21$ There is a separate cohort who can be neither assigned as Crohn's disease or UC, so are labelled as IBD unclassified.

Statement 26. Restorative proctocolectomy with an IPAA does not completely abolish the risk of neoplasia. We suggest that patients with high-risk factors may be offered surveillance pouchoscopy although it is unclear how frequently this should be performed. Low-risk patients do not require surveillance (GRADE: weak recommendation, very low-quality evidence. Agreement: $94.4 \%)$ 
Table 7 Montreal and Paris classification in Crohn's disease

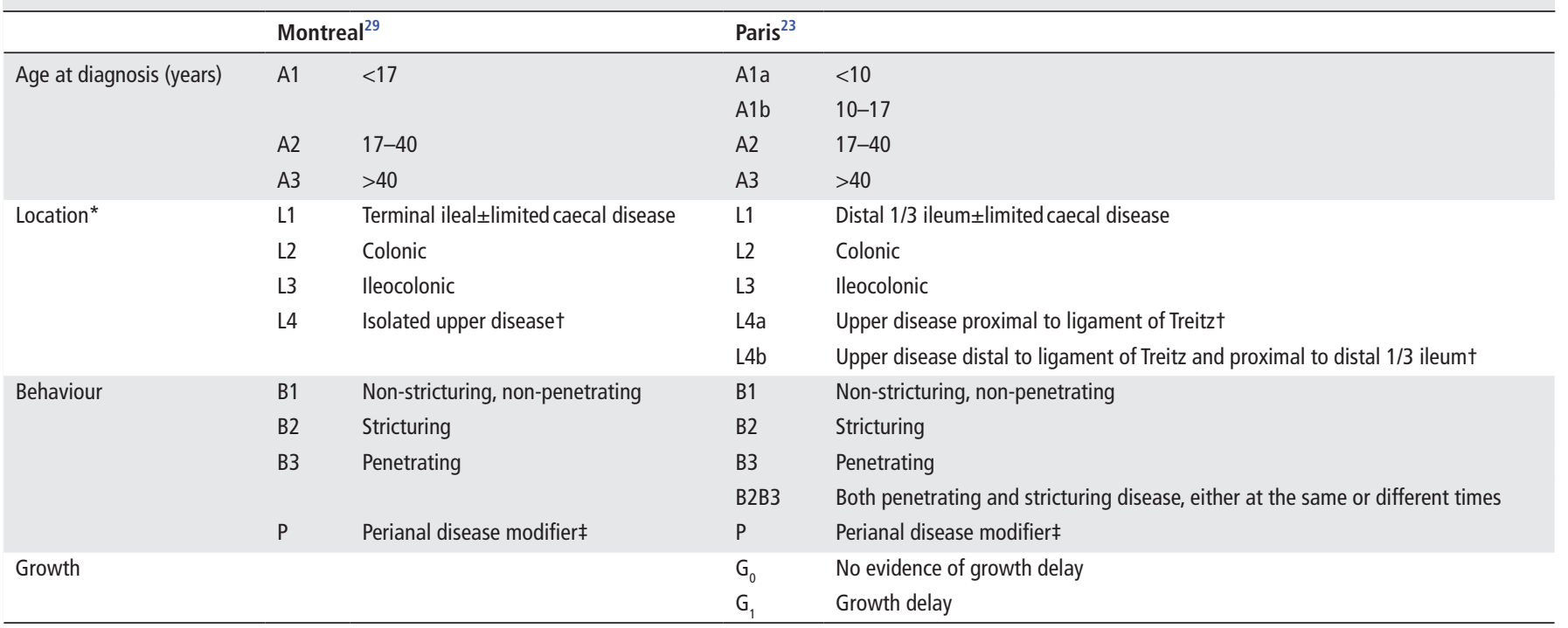

B2: Stricturing defined as the occurrence of constant luminal narrowing demonstrated by radiologic, endoscopic, or surgical examination combined with pre-stenotic dilatation and/or obstructive signs or symptoms but without evidence of penetrating disease.

B3: Penetrating disease: defined as the occurrence of bowel perforation, intra-abdominal fistulae, inflammatory masses and/or abscesses at any time in the course of the disease, and not secondary to postoperative intra-abdominal complications (excludes isolated perianal or rectovaginal fistulae).

*Defined as endoscopic or macroscopic extent.

tUpper disease in Montreal classification describes disease proximal to the terminal ileum. In both Montreal and Paris: L4 and L4a/b may coexist with L1, L2, L3.

†Perianal abscesses, ulcers or fistulae (but not skin tags or fissures).

\subsubsection{Phenotypic classification}

The Montreal classification ${ }^{29}$ is widely used to classify the key phenotypic features of patients with Crohn's disease. ${ }^{22}$ In order to capture the change in disease location and behaviour over time, and growth failure in the paediatric IBD phenotype, the Paris classification was developed as a modification of Montreal (table 7). ${ }^{23} 337338$ Using these classification systems in clinical practice is useful to ensure appropriate decision-making regarding surgery, medical treatment and surveillance. Montreal and Paris define disease extent according to endoscopic or macroscopic features. With an increasing emphasis on mucosal healing as a therapeutic target, it is important to note that histological evidence of inflammation may be more extensive than macroscopic features. This has been shown in paediatric studies, where inclusion of histological inflammation increases those classified with ileal involvement from 49\% to $71.3 \%$, those classified with Paris L3 disease increases by $10 \%$, those with Paris L3 + L4a by $24 \%$ and those with Paris L4 disease by $27 \% \cdot{ }^{339-341} \mathrm{~A}$ further approach to classifying disease relates to defining early disease: there is evidence that early treatment with biologics is more effective and an international consensus has proposed a Paris Definition for Early Crohn's Disease, defined as disease duration of 18 months or less after diagnosis without previous exposure to immunomodulators and/or biologics. ${ }^{337}$

\subsubsection{Clinical and endoscopic disease activity}

The Crohn's disease activity index (CDAI $)^{342}$ has in the past been used in clinical trials, ${ }^{343}$ but it has a number of limitations, including the parameters used to define remission (CDAI $<150$ ), and contemporary trial design no longer favours use of CDAI. ${ }^{344}$ In clinical practice, CDAI is cumbersome to calculate, requires diary data from patients, is weighted towards diarrhoea (which is often caused by factors other than inflammation), is not usable in patients with stomas and is not validated for use after surgery. In contrast, the Harvey Bradshaw Index (HBI) is both simple to calculate and measure, and less susceptible to confounding factors relying on clinical parameters only, yet is heavily weighted by diarrhoea. ${ }^{345}$ An HBI score $\leq 4$ is often used to define clinical remission. There is also value in monitoring patient-reported outcome measures (PROMs), reflecting the overall impact of disease on the individual. ${ }^{346}$ The IBD-Control PROM is an easy to use, reliable 8-item measure which correlates well with other quality of life measures ${ }^{347}$ and should be used to monitor patients' perspective of their disease. It should be noted that neither HBI nor PROMs measures correlate well with objective markers of inflammation in Crohn's disease, ${ }^{348}$ as subjective factors including psychological status impact significantly on patient-reported well-being. These measures are thus complementary to objective measures of disease activity obtained at endoscopy, imaging and surrogate measures including faecal calprotectin. More objective measures of disease impact including the Lemann index ${ }^{349}$ and endoscopic inflammation scores are more useful as treatment targets in clinical trials, ${ }^{350}$ and may drive therapeutic decision-making in clinical practice.

There are a number of endoscopic scoring systems which are used regularly in clinical trials $\mathrm{s}^{351352}$ although the two most commonly used are the Crohn's Disease Endoscopic Index of Severity (CDEIS) ${ }^{353}$ and the Simplified Endoscopic activity Score for Crohn's disease (SES-CD; table 8). ${ }^{354}$ While the CDEIS is complex to calculate, the SES-CD is a simple, reproducible and reliable endoscopic score. ${ }^{355} 356$ Both are used to assess for complete mucosal healing as an end-point in clinical trials. Although not used extensively in routine clinical practice, the SES-CD does systematise recording of features in each segment of the colon. Validated MRI activity scores are increasingly used as adjunct end-points in clinical trials. ${ }^{357} 358$ The Rutgeerts score (table 9) is used specifically to assess recurrent disease in the neo-terminal ileum after surgery. ${ }^{359}$ 


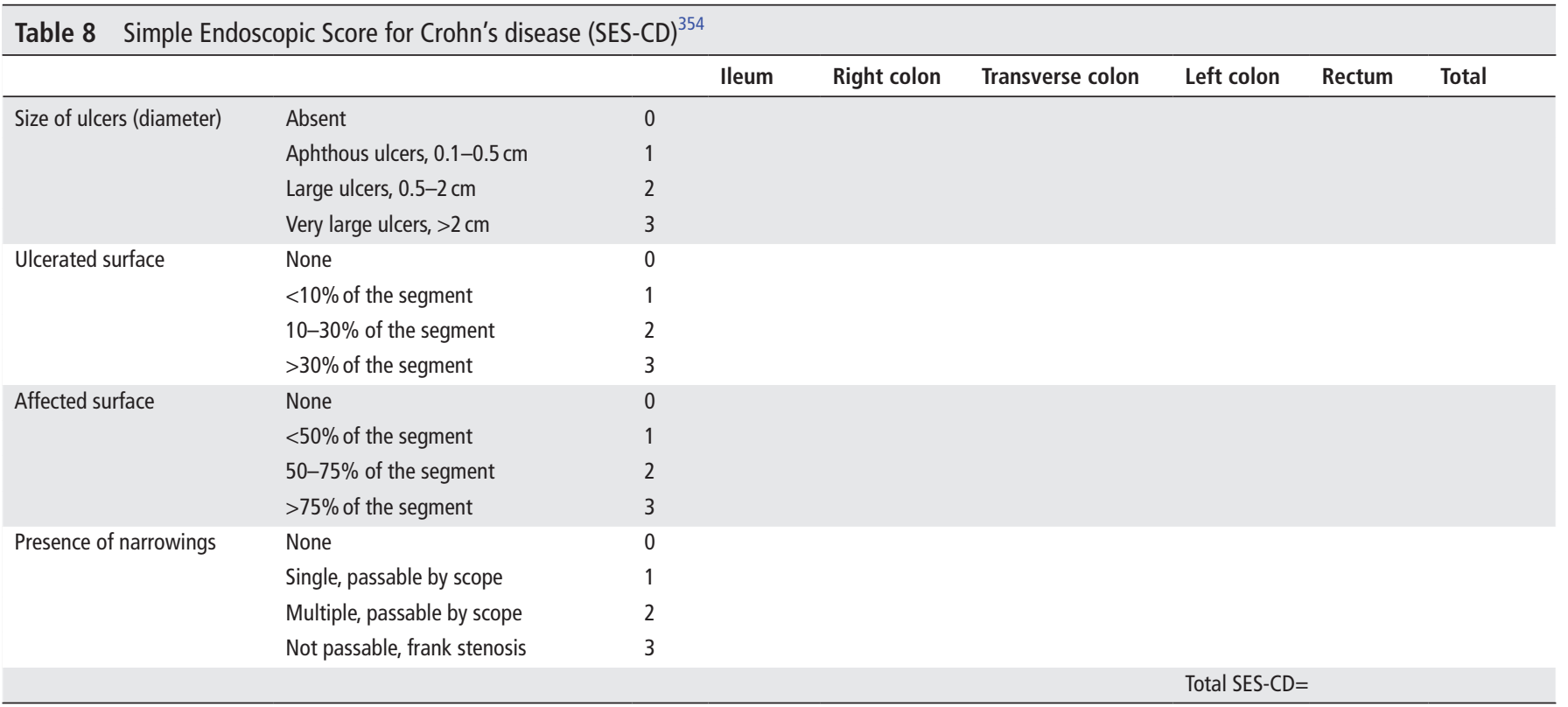

In clinical practice there is evidence that treatment escalation and de-escalation driven by objective markers of inflammation that are surrogates for endoscopy (CRP, faecal calprotectin), as well as clinical variables, can result in improved endoscopic and quality of life outcomes, at least in the short term. ${ }^{360} 361$ (The CALM trial is discussed further in the Section on Use of faecal calprotectin to inform therapy changes 5.5 .1 .2$)$. It seems likely that this will translate into long-term benefit, but as yet there is no widely agreed treatment target in Crohn's disease. As safer and more powerful therapies become available, it may be appropriate and feasible to use targets based on these objective measures in routine practice, in addition to clinical symptoms, to ensure effective control of the disease on a long-term basis. The choice of surrogate measure (such as faecal calprotectin), endoscopy, cross-sectional imaging or combinations of these at intervals may differ between patients, depending on their disease distribution, severity and their personal preference.

\subsubsection{Diagnostic tests}

Ileocolonoscopy with biopsy is established as the first-line investigation for suspected Crohn's disease. Ileoscopy with biopsy histology is superior in establishing the diagnosis of mild ileal Crohn's disease, however, intubation of the terminal ileum may not always be possible, and up to $20 \%$ of patients have isolated proximal small bowel disease beyond the reach of even complete ileocolonoscopy. For example, out of a cohort of 150 Crohn's disease patients, 36 had active small bowel disease diagnosed using CT enterography following a normal ileocolonoscopy. ${ }^{362}$ Ileoscopy and radiological imaging are complementary in diagnosis of ileal Crohn's disease. ${ }^{363} 364$ Dedicated small bowel

Table 9 Endoscopic evaluation of postoperative Crohn's disease recurrence at ileocolic anastomosis: Rutgeerts score ${ }^{359}$

\begin{tabular}{ll}
\hline $\begin{array}{l}\text { Endoscopic } \\
\text { remission }\end{array}$ & i1: no lesions in neo-terminal ileum \\
Endoscopic & i2: $>5$ aphthous ulcers \\
recurrence & $\begin{array}{l}\text { of larger lesions confined to ileocolonic anastomosis } \\
\text { i3: Diffuse aphthous ileitis with diffusely inflamed mucosa } \\
\end{array}$ \\
& i4: Diffuse inflammation with large ulcers, nodules and/or stenosis \\
\hline
\end{tabular}

imaging should be performed to complement ileocolonoscopy in all patients with suspected Crohn's disease and those with an unclassified colitis at ileocolonoscopy. Mucosal biopsy specimens are essential for a complete macroscopic and microscopic assessment of the colon and distal ileum. ${ }^{9365}$

Crohn's disease in the upper gastrointestinal tract has a prevalence up to $13 \%$ in some studies, ${ }^{366}$ and $16 \%$ in a more recent study of 119 patients. ${ }^{367}$ When found, it is usually accompanied by ileal or colonic disease. ${ }^{368}$ It has been suggested that upper GI endoscopy can help to differentiate between UC and Crohn's disease in patients with an unclassified colitis. Changes of focal gastritis occur, however, in UC as well as Crohn's disease, ${ }^{369}$ and so gastroscopy may not be helpful. If there is vomiting, dyspepsia or other upper GI symptoms then upper GI endoscopy is indicated, but not routinely in adults with suspected or proven Crohn's disease.

\subsubsection{Cross-sectional imaging: $C T, M R$ and small bowel ultrasound} Luminal barium fluoroscopic techniques have been the mainstay of radiological imaging for many years and have reasonable diagnostic accuracy for Crohn's disease. ${ }^{370}$ They have largely been replaced by cross-sectional imaging techniques which also provide information about the bowel wall and extra-enteric soft tissues, and thereby can better classify disease phenotype and behaviour. It is necessary to distend the bowel prior to CT or MRI using intraluminal contrast agents, ${ }^{371}$ with little data supporting invasive enteroclysis techniques over standard enterography, ${ }^{372}$ which is better tolerated by patients. Ultrasound can be performed without or with bowel distension using oral contrast agents. Several meta-analyses show no consistent difference in accuracy for Crohn's disease diagnosis between CT enterography (CTE), MR enterography (MRE) or small bowel ultrasound (SBUS), ${ }^{373-377}$ with sensitivity and specificity in the

Good Practice Recommendation 7. Suspected Crohn's disease should be investigated with ileocolonoscopy including segmental colonic and ileal biopsies (to look for microscopic disease); and with imaging to assess location and extent of small bowel disease (Agreement: 97.8\%). 
Statement 27. We suggest that adult patients with Crohn's disease do not routinely require upper gastrointestinal endoscopy as part of the diagnostic evaluation unless they have upper gastrointestinal symptoms (GRADE: weak recommendation, very low-quality evidence. Agreement: 91.1\%).

region of $85-95 \%$. However, a recent UK multicentre trial of 284 newly diagnosed or suspected relapse Crohn's disease patients showed that MRE had greater sensitivity for small bowel disease extent (presence and location) compared with SBUS (80\% vs $70 \%$ respectively). ${ }^{378} \mathrm{MRE}$ also had greater specificity than SBUS (95\% vs 81\% respectively), although SBUS had superior sensitivity to MRE for colonic disease presence in newly diagnosed patients (67\% vs $47 \%$ respectively). Diagnostic accuracy for abscess, fistulae and stenosis is also largely equivalent between techniques. ${ }^{376}$

\subsubsection{Detection of active disease}

Accuracy for detecting patients with active disease is generally reported to be over $85 \%,{ }^{376} 379$ although the data supporting SBUS is currently less consistent than for MRE and CT enterography $^{380}$ and multicentre trial data suggest MRE is significantly more sensitive than SBUS for detecting active small bowel disease (96\% vs $90 \%$ respectively). ${ }^{378}$ Radiological signs of disease activity include increases in bowel wall thickness and vascularity, contrast enhancement, T2 and diffusion weighted imaging signal (for MRE), and identification of ulceration and acute extraluminal complications. ${ }^{376}$ Validated and reproducible disease activity scores have been developed for MRE. 358381 Intravenous contrast-enhanced small bowel ultrasound shows promise $^{382}$ but there is as yet insufficient data to recommend first-line use.

\subsubsection{Investigation of strictures}

Data supporting the use of imaging to quantify fibrosis are limited $^{383}$ and the topic is under active investigation, with novel MRI magnetisation transfer sequences, ${ }^{384}$ delayed contrast enhancement, ${ }^{385}$ contrast enhanced ultrasound ${ }^{386}$ and ultrasound elastography ${ }^{387}$ all showing promise. Ultrasound detection of strictures may be improved by oral contrast. ${ }^{377}$

Compared with an age-matched population, Crohn's disease patients have a 2-3-fold increased incidence of colorectal cancer, while the risk of small bowel malignancy is increased between 18 and 27 times. $^{388389}$ Up to $3.5 \%$ of Crohn's disease strictures may be complicated by dysplasia or malignancy ${ }^{390}$ so endoscopic biopsy of accessible strictures should be performed to achieve a pathological diagnosis.

\subsubsection{Radiation exposure}

Diagnostic imaging, particularly CT scanning, can expose Crohn's disease patients to doses of ionising radiation which

Statement 28. We suggest that cross-sectional imaging, specifically MRI, CT and ultrasound, have largely replaced conventional barium fluoroscopic and nuclear medicine techniques and have the advantage of evaluating both luminal and extraluminal disease. Emphasis should be placed on MR enterography and ultrasound as they do not expose patients to ionising radiation (GRADE: weak recommendation, moderatequality evidence. Agreement: 97.9\%).
Statement 29. We suggest that, while there is no clear evidence of diagnostic superiority for one cross-sectional imaging modality over another for stricture diagnosis, emphasis should be placed on techniques that do not expose patients to ionising radiation. For colonic or anastomotic strictures, endoscopy and biopsy should be performed to assess and exclude cancer, provided the stricture is accessible and endoscopy deemed safe (GRADE: weak recommendation, very low-quality evidence. Agreement: 100\%).

may significantly increase their lifetime risk of cancer. ${ }^{391-393}$ This risk is particularly important for children and young people. ${ }^{394}$ Crohn's disease patients have more than twice the radiation exposure compared with UC patients. ${ }^{395}$ A study of 409 patients from a tertiary hospital showed that $15.5 \%$ had a cumulative exposure dose in excess of $75 \mathrm{mSv}^{392}$ (this dose is considered to increase the risk of cancer mortality by $7.3 \%$ ). Factors associated with excessive diagnostic radiation exposure included age under 17 at diagnosis, upper gastrointestinal disease location, penetrating disease, need for intravenous corticosteroids and more than one Crohn's disease surgical operation. Although significant dose reductions are now possible with emerging CT image reconstruction techniques, ${ }^{396} 397$ MRE and small bowel ultrasound are generally preferred over CT to limit patient exposure to ionising radiation.

\subsubsection{Capsule endoscopy}

Capsule endoscopy provides high resolution endoluminal images of the small bowel, is less invasive than conventional endoscopic techniques and is usually well tolerated by patients. ${ }^{398}$ Although recent randomised trial data suggest a clear fluid preparation may be sufficient, marginally outperforming active bowel preparation, ${ }^{399}$ patients are commonly given bowel preparation. ${ }^{400}$ A recent European expert technical review assessing available meta-analysis data recommends the use of PEG bowel preparation to improve visualisation, but notes inconclusive evidence on the impact of preparation on completion rates and diagnostic yield. ${ }^{401}$ The same technical review also recommends use of an anti-foaming agent prior to capsule ingestion to improve visualisation. $^{401}$

Evaluating diagnostic accuracy of capsule endoscopy is hampered by a lack of reference standard and many studies instead report the 'diagnostic yield'. In this regard, meta-analysis data suggest the diagnostic yield of capsule endoscopy in suspected Crohn's disease is significantly greater than barium fluoroscopy (52\% vs 16\%), CT enterography (68\% vs $21 \%$ ) and ileocolonoscopy (47\% vs 25\%). ${ }^{381}$ In established Crohn's disease it was significantly greater than push enteroscopy $(66 \%$ vs $9 \%$ ), barium fluoroscopy (71\% vs 36\%) and CT enterography (71\% vs $39 \%) .{ }^{402}$ Data suggest capsule endoscopy is superior to

Statement 30. We suggest that capsule endoscopy has greater sensitivity for mucosal small bowel Crohn's disease than radiological imaging techniques and can generally be performed where inflammatory small bowel disease is still suspected despite normal or equivocal cross-sectional imaging. Minor changes found at capsule endoscopy may be insufficient in isolation to diagnose Crohn's disease, particularly with recent NSAID use (GRADE: weak recommendation, moderate-quality evidence. Agreement 90.9\%). 
Statement 31. The risk of capsule retention is low in suspected Crohn's disease patients without symptoms suggestive of stricture. However, when obstructive symptoms are present, or in known stricturing Crohn's disease where capsule endoscopy is indicated, we suggest that patency capsule should precede capsule endoscopy (GRADE: weak recommendation, low-quality evidence. Agreement: 93.5\%).

MRE at detecting small bowel inflammation in Crohn's disease, particularly for proximal or superficial small bowel lesions, ${ }^{370}$ although a recent meta-analysis did not find any statistically significant difference in diagnostic yield between MRE and capsule endoscopy. ${ }^{370}$

The utility of diagnostic yield as a surrogate for diagnostic accuracy has been criticised ${ }^{403}$ with specificity for capsule endoscopy reported as 53\% in one study, using a consensus reference standard paradigm. ${ }^{404}$ Minor mucosal abnormalities found at capsule endoscopy can be seen in normal individuals, particularly those who have been using NSAIDs, ${ }^{398} 405$ and while validated diagnostic criteria for capsule endoscopy diagnosis of Crohn's disease are in development, they are not currently in widespread clinical use. ${ }^{398} 405$

Use of capsule endoscopy should therefore be restricted to those with a high clinical suspicion of Crohn's disease (suggestive clinical picture and raised faecal calprotectin) and abstinence from NSAID ingestion for at least 1 month, ${ }^{405}$ where cross-sectional imaging has been normal or equivocal.

The size and rigidity of capsules used for capsule endoscopy mean they can be retained within the small bowel, usually in stricturing disease. The risk of capsule retention is lower in patients with suspected Crohn's disease than in those with established disease. In a 2017 meta-analysis the risk of capsule retention in suspected Crohn's disease (nine studies, 968 patients) was $3.6 \%$ (95\% CI $1.7 \%$ to $8.6 \%$ ), although there was considerable heterogeneity between included studies. ${ }^{406}$ In established Crohn's disease (11 studies, 558 patients), the risk of capsule retention was $8.2 \%$ (95\% CI $6 \%$ to $11 \%)$. ${ }^{406}$ Retention after successful patency capsule passage or exclusion of strictures by cross-sectional imaging was $2.7 \%$ (95\% CI 1.1 to 6.4). Comparative data regarding cross-sectional imaging and patency capsule is limited. ${ }^{401}$ When capsule endoscopy is indicated in patients with known stricturing Crohn's disease or those with suspected strictures, including patients presenting with abdominal pain or distention, nausea or vomiting, or a history of small bowel resection, abdominal/pelvic radiation exposure or chronic NSAID use, the use of preceding patency capsule is suggested. ${ }^{401405407}$

\subsubsection{Balloon-assisted enteroscopy}

Device-assisted endoscopy such as balloon-assisted enteroscopy can visualise the small bowel mucosa beyond the reach of ileocolonoscopy, allowing tissue biopsy for histological assessment. The diagnostic yield may be similar to capsule endoscopy, with a recent systematic review reporting a yield of $63.4 \%(95 \%$ CI $42 \%$ to $82.3 \%$ ) in known or suspected Crohn's disease, ${ }^{408}$ with likely greater sensitivity than MRE. ${ }^{409}$ The procedures are however costly, invasive, requiring deep sedation or general anaesthesia, and not without risk. A recent meta-analysis ${ }^{410}$ reported a perforation rate of $0.15 \%$ (95\% CI $0.05 \%$ to $0.45 \%$ ) for diagnostic balloon-assisted enteroscopy, and an overall major complication rate (including perforation and bleeding) of $0.72 \%$ (95\% CI $0.56 \%$ to $0.90 \%$ ) has been reported. ${ }^{408}$
Statement 32. We recommend that mild to moderate ileocaecal Crohn's disease can be treated with ileal-release budesonide $9 \mathrm{mg}$ once daily for 8 weeks to induce remission (GRADE: strong recommendation, high-quality evidence. Agreement: 100\%).

Given the high diagnostic accuracy of alternative less invasive tests, notably cross-sectional imaging and capsule endoscopy, diagnostic device-assisted techniques should be reserved for patients with high clinical suspicion of Crohn's disease despite negative ileocolonoscopy, who have suspicious but not diagnostic cross-sectional imaging or capsule endoscopy, particularly if findings would alter therapeutic strategy, or in patients where a firm histological diagnosis would alter management. ${ }^{405}$ Device-assisted enteroscopy does have a role in established Crohn's disease where therapeutic intervention, such as stricture dilatation, is needed. ${ }^{411}$

\subsection{Induction of remission in mild to moderate disease ileocolonic disease}

\subsubsection{Budesonide in ileocaecal Crohn's disease}

In a randomised double-blind trial, ileal-release budesonide $9 \mathrm{mg}$ once daily for 8 weeks was as effective as prednisolone ( $40 \mathrm{mg}$ daily tapering to $5 \mathrm{mg}$ at 8 weeks) at inducing remission in patients with mild-to-moderate ileocaecal Crohn's disease with treatment efficacy (CDAI score <150) of $51 \%$ on budesonide at 8 weeks compared with $52.5 \%$ for prednisolone, ${ }^{412}$ and significantly fewer side effects. Multiple meta-analyses have also demonstrated the efficacy of budesonide $9 \mathrm{mg} /$ day over placebo in inducing remission. ${ }^{413-415}$ A once-daily $9 \mathrm{mg}$ dose is as effective as $3 \mathrm{mg}$ three times daily. ${ }^{416}$ In the context of more severe disease (CDAI >300) budesonide is inferior to prednisolone (RR $0.52,95 \%$ CI 0.28 to 0.95$).{ }^{414}$ When remission has been achieved, budesonide should be tapered over 1-2 weeks.

\subsubsection{Corticosteroids in colonic Crohn's disease}

In mild, moderate and severe colonic Crohn's disease, systemic corticosteroids such as prednisolone are effective in inducing remission. ${ }^{417-419}$ A starting dose of $40 \mathrm{mg}$ tapering by $5 \mathrm{mg}$ weekly is often used, but should be tailored to disease severity and patient tolerance. Ileal-release budesonide does have benefit in Crohn's disease affecting the proximal colon, ${ }^{412}$ but there is no evidence of benefit in more distal colonic inflammation. There are no trials of colonic-release budesonide-MMX in Crohn's disease at present.

\subsubsection{Nutritional therapy}

\subsubsection{Exclusive Enteral Nutrition (EEN)}

Meta-analysis has shown that EEN is as effective as corticosteroids at inducing remission in $73 \%$ of paediatric patients on an intention to treat basis ${ }^{420}$ but not in adults. ${ }^{421}$ In paediatrics, it is considered the primary treatment option to induce disease remission, ${ }^{420}$ and has added value in that it not only improves nutritional status but also benefits growth. In adults, although

Statement 33. We recommend that active Crohn's colitis can be treated with an 8-week course of systemic corticosteroids to induce remission (GRADE: strong recommendation, high-quality evidence. Agreement: 100\%). 
Statement 34. We suggest that Exclusive Enteral Nutrition (EEN) may be used to induce remission in mild to moderate Crohn's disease patients where avoidance of corticosteroid is desired, and in those who are motivated to adhere strictly to EEN for up to 8 weeks (GRADE: weak recommendation, very low-quality evidence. Agreement: 86.4\%).

studies have been small and underpowered, there does seem to be a consistent message that, where tolerated, EEN can be effective at inducing remission ${ }^{42-425}$ even in the presence of complications. ${ }^{426}$ There is increasing evidence that EEN can alter the microbiome, with differences in those who have a long-term response. $^{427}$

Tips on the use of EEN are shown in box 3. In practice, EEN is not frequently used in adult populations. This is reportedly due to a lack of high-quality evidence for its use ${ }^{428}$ and limited access to dietetic expertise within an IBD multidisciplinary team (MDT). ${ }^{429}$ Where avoidance of corticosteroids is considered appropriate and patients are motivated to try dietary alternatives, EEN should be considered with appropriate dietetic support. ${ }^{421} 429430$

Ten days of EEN can achieve symptomatic relief; however mucosal healing takes longer, up to 8 weeks. ${ }^{431-433}$ Further research is needed to identify the optimum duration, but 4-6 weeks of EEN is considered the minimum. ${ }^{429} 434$

EEN given as elemental diets are not palatable, therefore polymeric (whole protein) and semi-elemental (peptide) based feeds were created to overcome this barrier and have been shown to be as effective as elemental diets. ${ }^{435}$ EEN can be taken orally in the majority of patients, however the nasogastric route can be considered as an alternative where appropriate. ${ }^{436}$ A Cochrane review showed no difference in outcomes between elemental and non-elemental feeds, but evidence quality was very low. ${ }^{421}$

\subsubsection{Elimination diets}

There is much interest in elimination diets being used to induce or maintain disease remission. Following a course of EEN, patients can be unsure what foods to add back into their diet first. A low fat, low fibre, exclusion (LOFFLEX) diet was developed to provide a dietary intervention for 2-4 weeks based on a small number of foods unlikely to trigger symptoms (an exclusion diet) followed by gradual food reintroduction with a new food every few days. The LOFFLEX diet has been shown to maintain disease remission in $56 \%$ of patients at 2 years, ${ }^{437}$ but is not used widely. Another study in paediatrics used a 3-day rapid food reintroduction diet compared with a 5-week food reintroduction plan and showed similar relapse rates of almost $50 \%$ of patients at 12 months indicating that food can be reintroduced more quickly than traditionally thought. ${ }^{438} \mathrm{~A}$ variety of other diets have been proposed in this context, but they lack the backing of high-quality evidence. ${ }^{428}$ The recently reported Crohn's disease treatment with eating (CD-TREAT) study explored the possibility of replicating EEN with solid foods. ${ }^{439}$ Both EEN and the experimental diet achieved similar

Statement 35. We recommend that polymeric feeds are as effective as elemental or semi-elemental feeds in treatment of Crohn's disease, and improve adherence (GRADE: strong recommendation, very low-quality evidence. Agreement: $86.7 \%$ ).
Box 3 Practical guide for Exclusive Enteral Nutrition

(EEN) to induce remission in Crohn's disease

- Counsel patients on the risks and benefits of all available treatment options including EEN.

- EEN is provided as a prescribed liquid diet excluding all food and drink except still water. Some units allow limited optional intake beyond this, but there is little evidence supporting what foods or drinks can be added without impacting efficacy. Permit Crusha flavouring (now sugar-free), Fox's Glacier Mints, sugar-containing 7-Up and black coffee.

- Whole protein, peptide or elemental diets are equally efficacious but whole protein feeds are more palatable and are more likely to be tolerated.

- EEN is nutritionally complete with all relevant micronutrients and trace elements.

- Limited palatability and tolerance are often reasons for failure, so encouragement from the whole MDT is important for success. This is best achieved with a formalised MDT pathway for EEN management and specific points of contact to assess progress.

- A starter regimen, increasing the prescribed daily volume gradually over a few days while reducing food intake, is important to build up tolerance and prevent the risk of refeeding, especially in patients where dietary intake has been suboptimal beforehand or weight loss has been significant.

- Bloods for refeeding syndrome include urea and electrolytes (for potassium), phosphate and magnesium, and should be monitored daily while calorie intake is increased to maximum in at-risk patients.

- A standard target regimen should be based on requirements for energy: $25-30 \mathrm{kcal} / \mathrm{kg} /$ day and protein: $1 \mathrm{~g} / \mathrm{kg} /$ day. Nonstandard regimens may be used where refeeding syndrome is a risk (with lower calories), or where catch-up nutrition is required.

- Once the target regimen is met, EEN should be continued for 6-8 weeks to induce mucosal healing.

- Once EEN is established, the vast majority of patients can continue with their usual daily activities.

- Most adult patients can tolerate EEN orally, however nasogastric feeding may be required if target volumes cannot be met orally or where feed tolerance is limited with boluses.

- Regular monitoring via email or telephone will help to maintain adherence.

MDT, Multidisciplinary team.

microbiome effects in healthy volunteers and rats, as well as improved HLA-B27 rat ileitis histopathology scores. In addition, the same paper reported a non-blinded study of five children with relapsing Crohn's disease in which the experimental diet achieved $3 / 5$ remission and $4 / 5$ response by weighted paediatric CDAI. ${ }^{439}$ This is an area that requires further investigation before it can be considered a standard therapy.

\subsubsection{Antibiotic therapy}

Trials of antibiotic therapy in Crohn's disease have studied a wide range of antimicrobial agents. While a meta-analysis demonstrated efficacy for these pooled trials over placebo (RR for continued disease activity 0.85 (95\% CI 0.73 to 0.99 ), 
$p=0.03),{ }^{440}$ there was such heterogeneity in the agents and dosing regimens used that it makes it difficult to draw meaningful conclusions.

The risk of adverse effects, particularly with prolonged or repeated courses, should also be taken into consideration. In this regard, rifaximin (a non-absorbed oral antibiotic), in an extended-intestinal release formulation has been shown in a large dose-ranging study to be effective, although at a higher dose than usually employed $(800 \mathrm{mg} \mathrm{BD})$. However, there was no dose-response, with numerical but not statistical difference to placebo for the $400 \mathrm{mg} \mathrm{BD}$ and $1200 \mathrm{mg}$ BD dose. ${ }^{441442}$ Rifaximin is unlicensed for this indication.

In a blinded randomised trial in 73 patients with paediatric Crohn's disease, azithromycin $75 \mathrm{mg} / \mathrm{kg} 5$ days per week for 4 weeks, then metronidazole $20 \mathrm{mg} / \mathrm{kg} /$ day for another 4 weeks, compared with metronidazole only for 8 weeks, showed a remission rate of $66 \%$ for azithromycin/metronidazole and $39 \%$ for metronidazole $(\mathrm{p}=0.025)$, although response rates were similar. In 12 patients failing metronidazole, $83 \%$ achieved remission on open-label azithromycin. ${ }^{443}$ There is continuing research interest in antibiotic therapy in Crohn's disease treatment, but at present antibiotics should only be used in patients with disease complicated by infection (abscesses, bacterial overgrowth, Clostridium difficile) or perianal fistulising disease. Overall the efficacy for antibiotics as induction therapy in mild-moderate Crohn's disease remains unproven.

The role of Mycobacterium avium subspecies paratuberculosis (MAP) has been debated for many years. A randomised placebo-controlled trial of 2 years therapy with clarithromycin, rifabutin and clofazimine in 213 patients with active Crohn's disease, with a further year of follow-up, showed early benefit of antibiotics, but overall there was no significant difference in relapse rate during follow-up. ${ }^{444}$ There was debate about the dosage and formulation chosen, and the study design (with withdrawal of non-responders at week 16). A further phase 3 trial using higher doses was reported in October $2018 .{ }^{445}$ A total of 331 patients with moderate to severely active Crohn's disease were randomised to RHB-104 (clarithromycin $95 \mathrm{mg}$, rifabutin $45 \mathrm{mg}$ and clofazimine $10 \mathrm{mg}$ ) five capsules twice daily or placebo for 52 weeks, in addition to their pre-study therapy. At week 26 , remission was achieved in $37 \%$ versus $23 \%$ on placebo $(\mathrm{p}=0.007)$, and durable remission (from week 16 to 52$)$ was achieved in $18 \%$ versus $9 \%$ on placebo $(\mathrm{p}=0.019)$. No data are available on endoscopic healing or on persistence of benefit after stopping treatment.

\subsubsection{Surgery in localised ileocaecal Crohn's disease}

The LIR!C study compared laparoscopic ileocaecal resection $(n=73)$ to infliximab $(n=70)$ for patients with terminal ileal disease in whom conventional therapy had failed. ${ }^{446}$ Across a number of comparators there was equivalence between the two approaches, suggesting that resection is a reasonable alternative to medical therapy in this scenario. The LIR!C trial excluded those who had previous resection, a small bowel segment longer than $40 \mathrm{~cm}$, abscesses or where surgery was deemed likely due to pre-stenotic dilatation. During the median follow-up period of 4 years (range 2-6), approximately a third of patients $(n=26$, $39 \%)$ in the infliximab group required surgery while a quarter of patients in the surgery group $(n=19,26 \%)$ required infliximab. Taken together, these data suggest resection is a cost-effective intervention based on the cost of originator infliximab. ${ }^{447}$ Present day medical costs should be lower with the widespread availability of biosimilar infliximab.
Statement 36. We suggest that laparoscopic resection should be considered in localised ileocaecal Crohn's disease for those failing or relapsing after initial medical therapy, or in those preferring surgery to continuation of drug therapy (GRADE: weak recommendation, moderate-quality evidence. Agreement: $93.5 \%)$.

\subsection{Initial treatment of moderate to severe ileocolonic Crohn's disease}

\subsubsection{Corticosteroids}

Systemic corticosteroids are still an effective initial therapy for moderate to severely active Crohn's disease, ${ }^{418} 419$ regardless of disease location, but every effort should be made to limit exposure. ${ }^{448}$ For less severe symptoms in those with ileocaecal disease location, ileal-release budesonide may be tried initially, but if ineffective, prednisolone may be required. For teenagers, particularly where growth has not completed, and for patients with diabetes or steroid intolerance, alternatives can be considered, including EEN.

\subsubsection{Early use of biological therapy}

In patients with an aggressive disease course or 'high-risk' poor prognostic factors, the early introduction of biologics may be considered. High-risk features include: complex (stricturing or penetrating) disease at presentation; perianal fistulising disease; age under 40 years at diagnosis; and need for steroids to control the index flare. ${ }^{449} 450$ The predictive power of these features is limited. There is great interest in biomarkers that identify a Crohn's disease subgroup at high risk of an aggressive disease course. A gene expression signature from peripheral CD8 $\mathrm{T}$ cells in active untreated IBD has been identified, ${ }^{451}$ and is now being tested using a whole blood assay in randomised controlled trials of a 'top-down' versus accelerated 'step-up' therapy.

The decision to start biological therapy should also consider factors such as stage of life, work absence and availability of other treatment options. Discussion in a multidisciplinary team meeting can be helpful. As well as anti-TNF therapy, vedolizumab and ustekinumab can be considered as first-line biologics.

\subsection{Maintenance treatment in ileocolonic Crohn's disease}

Irrespective of whether remission is induced surgically or medically, corticosteroids are not effective in maintaining remission in Crohn's disease compared with placebo (see Section 5.2.8.1: Overuse of corticosteroids). Systematic reviews and pooled trial data show that budesonide does not reduce relapse rates over a 12-month period. ${ }^{452-454}$ One systematic review did show a modest reduction in CDAI scores, but this was outweighed by a significant increase in adverse events and adrenocortical suppression compared with the placebo groups. ${ }^{454}$

Statement 37. We recommend that moderate to severely active uncomplicated luminal Crohn's disease should be treated initially with systemic corticosteroids (GRADE: strong recommendation, high-quality evidence), but we suggest that those with extensive disease or other poor prognostic features should be considered for early introduction of biological therapy (GRADE: weak recommendation, moderate-quality evidence. Agreement: $86.7 \%)$. 
Statement 38. We recommend that systemic or locally acting corticosteroids should be avoided as maintenance therapy in Crohn's disease due to toxicity and lack of efficacy (GRADE: strong recommendation, high-quality evidence. Agreement: $100 \%)$

\subsubsection{Immunomodulator therapy}

Relapse of Crohn's disease is common on corticosteroid withdrawal, particularly in moderate to severe disease, and early initiation of corticosteroid-sparing therapy is appropriate. Immunomodulators such as azathioprine, mercaptopurine or methotrexate are effective in the maintenance of remission of Crohn's disease.

\subsubsection{Thiopurine therapy}

Thiopurines should not be used for induction of remission in active Crohn's disease. Thiopurines are more effective than placebo in maintenance of remission in Crohn's disease but the Cochrane analysis reports low quality evidence $(\mathrm{NNT}=9) .{ }^{455}$ A systematic review and network meta-analysis also showed the benefit of azathioprine/mercaptopurine compared with placebo in remission maintenance (OR 1.7 (95\% CI 1.3 to 2.6)), although anti-TNF therapy was significantly more effective than thiopurines. ${ }^{456}$

\subsubsection{Methotrexate}

Methotrexate should not be used as monotherapy for induction of remission, but may be used in Crohn's disease patients failing to respond to corticosteroids. The landmark trial evaluated intramuscular methotrexate $25 \mathrm{mg}$ weekly given to patients with chronic active Crohn's disease despite at least 3 months of prednisolone. It showed increased clinical remission rates compared with placebo at 16 weeks, with reduced prednisolone requirements. 457458

A follow-on study also showed efficacy of methotrexate as a maintenance therapy, ${ }^{459}$ a finding which has been confirmed in a recent Cochrane review. ${ }^{460}$ A network meta-analysis and systematic review showed the benefit of methotrexate with ORs of 2.4 (95\% CI 1.1 to 4.8$).^{456}$

In other disease areas there is convincing evidence of the superiority of parenteral administration of methotrexate over the oral route. ${ }^{461} 462$ As a result, a variety of studies have attempted to compare the efficacy of different routes of administration for methotrexate in Crohn's disease. A retrospective cohort study of 118 patients showed benefit of methotrexate in both induction and maintenance treatment for Crohn's disease, with steroid-free remission being achieved in $37.2 \%$ of patients, and maintained relapse-free in $63.6 \%$ for a median of 12 months. ${ }^{463}$ A multicentre retrospective study in 226 children showed numerical, but not statistical superiority of subcutaneous methotrexate over the oral route, with sustained corticosteroid-free remission in 32\% for subcutaneous versus $26 \%$ for

Statement 39. We recommend that for patients with moderate to severe Crohn's disease responding to prednisolone, early introduction of maintenance therapy with thiopurines (GRADE: strong recommendation, low-quality evidence) or methotrexate (GRADE: strong recommendation, moderate-quality evidence) should be considered to minimise risk of flare as prednisolone is withdrawn (Agreement: 93.3\%).
Statement 40 . We recommend that azathioprine or mercaptopurine can be used as monotherapy in the maintenance of remission in Crohn's disease (GRADE: strong recommendation, low-quality evidence. Agreement: 100\%).

oral (OR $1.7,95 \%$ CI 0.5 to 5.9$).{ }^{464}$ There was no difference in nausea or abnormal liver enzymes between the two routes of administration.

Oral methotrexate has reduced and extremely variable bioavailability (not corrected by use of folic acid) compared with parenteral administration, particularly at doses above 15 mg. ${ }^{465}{ }^{466}$ Because bioavailability and pharmacokinetics are very similar for subcutaneous and intramuscular methotrexate, the subcutaneous route should be preferred to intramuscular as it is easier and less painful. ${ }^{467}$

Induction therapy should be given by the subcutaneous route. If patients prefer, this can be switched to oral during the maintenance phase, with the option to switch back to subcutaneous if effectiveness is lost. Whatever the route of administration, methotrexate should be given with folic acid to reduce gastrointestinal and liver toxicity, $5 \mathrm{mg}$ weekly (traditionally 1-2 days after the methotrexate dose) or $1 \mathrm{mg}$ daily.

\subsubsection{Mesalazine}

UK data from 1990 to 2010 show over half of patients with Crohn's disease were prescribed 5-ASA, ${ }^{468}$ and Swiss data show it is more often given for Crohn's colitis. ${ }^{469}$ A Cochrane systematic literature review showed that oral 5-ASA has no efficacy in maintaining clinical remission in Crohn's disease, ${ }^{470}$ with similar negative findings in meta-analyses for induction or maintenance. ${ }^{413} 414471472$ A recent review of colonic Crohn's disease $^{473}$ showed that there was no benefit for 5-ASA in colonic Crohn's disease, but two studies have shown possible benefit for sulphasalazine in remission induction. ${ }^{418} 419$ Thus, 5-ASAs are not recommended for induction or maintenance treatment of Crohn's disease.

\subsubsection{Biological therapy with anti-TNF drugs, vedolizumab or ustekinumab}

\subsubsection{Infliximab}

Infliximab is a monoclonal antibody to tumour necrosis factor alpha (TNF) and was the first in class to be used in IBD, demonstrating definitive benefit in luminal Crohn's disease in the ACCENT I study. 573 patients with active luminal disease received a single $5 \mathrm{mg} / \mathrm{kg}$ intravenous dose, and after assessment of response at week 2, were randomly assigned to infusions of placebo at weeks 2, 6 and then 8-weekly (group 1), or infliximab $5 \mathrm{mg} / \mathrm{kg}$ at the same time points, or $5 \mathrm{mg} / \mathrm{kg}$ at weeks 2 and 6 , then $10 \mathrm{mg} / \mathrm{kg} 8$-weekly. At week 2, 58\% responded to the initial infusion, and of these responders: at week 30,39\% treated with $5 \mathrm{mg} / \mathrm{kg}$ maintenance and $45 \%$ on $10 \mathrm{mg} / \mathrm{kg}$ were in clinical

Statement 41. We suggest that methotrexate may be used for the maintenance of remission of Crohn's disease, and the dose should be at least $15 \mathrm{mg}$ weekly. Subcutaneous administration has better bioavailability than oral, particularly at higher doses (GRADE: weak recommendation, moderate-quality evidence. Agreement: 88.4\%) 
Statement 42. We recommend that mesalazine is not used for induction or maintenance of remission in Crohn's disease (GRADE: strong recommendation, moderate-quality evidence. Agreement: 97.7\%).

remission, with similar remission rates observed at week $54 .^{474}$ In routine clinical practice reported results are significantly better, with a large single-centre cohort of 614 Crohn's disease patients (treated for luminal, perianal or extraintestinal manifestations) showing that $89.1 \%$ had clinical improvement after initial treatment and $63.4 \%$ showing sustained clinical benefit. ${ }^{475}$ This study included a significant proportion of patients receiving episodic therapy, whereas regular scheduled therapy is of proven superiority. ${ }^{476}$

4.4.3.1.1 Combination therapy of infliximab with an immunomodulator The SONIC study showed that combined infliximab and azathioprine was superior to infliximab in achieving clinical remission and mucosal healing. ${ }^{477}$ A network meta-analysis of published data shows that combination therapy was more effective than azathioprine monotherapy, as was adalimumab monotherapy. ${ }^{456}$ Similar benefits of combination therapy are seen in children. ${ }^{478} 479$ Addition of an immunosuppressant has also been shown to reduce the need for dose escalation of infliximab and also reduces the rate of drug switching. ${ }^{480}$

In the PANTS 3-year observational cohort of 1601 Crohn's patients treated with infliximab or adalimumab, 751 patients were treated with infliximab. ${ }^{481}$ At week 54 the immunogenicity rates for Remicade and biosimilar infliximab (Inflectra/ Remsima) were $26 \%$ and $28 \%$, respectively. Immunomodulator use reduced the risk of immunogenicity in infliximab therapy $(\mathrm{HR}=0.37, \mathrm{p}<0.0001)$.

A study of patients who had recently started prednisolone treatment for active disease showed that combination therapy with infliximab and methotrexate was no more effective that infliximab monotherapy in maintaining remission up to 50 weeks, although equally safe. ${ }^{482}$ A recent Cochrane systematic literature review evaluating this ${ }^{460}$ and a further small openlabel study ${ }^{483}$ reached the same conclusion. Immunogenicity to infliximab may, however, be reduced by the addition of methotrexate, ${ }^{480}$ suggesting that some clinical benefit might have been observed beyond the 1 year timeframe of the study. A paediatric registry study of 502 Crohn's disease patients studied the impact of concomitant immunomodulator therapy on the duration of infliximab therapy. ${ }^{484}$ Concomitant methotrexate, taken for more than 6 months, increased likelihood of remaining on infliximab, both compared with non-use of immunomodulators and compared with thiopurine use. Due to the small number of girls given methotrexate, only boys were included in this analysis.

In order to maximise the benefit of infliximab therapy and reduce treatment failure, combination therapy with

Statement 43 . We recommend that patients refractory to immunomodulator therapy despite dose optimisation should be considered for biological therapy. Choice between anti-TNF therapy, ustekinumab and vedolizumab should be made on an individual basis, considering patient preference, cost, likely adherence, safety data and speed of response to the drug (GRADE: strong recommendation, very low-quality evidence. Agreement: 95.7\%).
Statement 44. We recommend that combination therapy of infliximab with a thiopurine should be used as it is more effective than monotherapy infliximab in induction and maintenance of remission in active Crohn's disease (GRADE: strong recommendation, high-quality evidence. Agreement: $97.7 \%$ ).

immunomodulator should always be preferred (with stronger evidence for azathioprine than methotrexate). For those intolerant to thiopurines and methotrexate, alternatives to infliximab should be used unless there are other compelling reasons (such as the presence of perianal disease).

\subsubsection{Adalimumab}

Adalimumab is a monoclonal antibody to TNF administered subcutaneously. The CLASSIC I study in moderate to severe Crohn's disease naïve to anti-TNF therapy showed that the optimum dose for induction therapy was $160 \mathrm{mg}$ followed by $80 \mathrm{mg}$ at week 2, with remission (CDAI < 150) achieved in 36\% $(\mathrm{p}=0.001$ against placebo) compared with $24 \%$ (80 mg/40 mg), $18 \%\left(40 \mathrm{mg} / 20 \mathrm{mg}\right.$ ) and $12 \%$ on placebo. ${ }^{485}$ In the CHARM study of maintenance therapy, responders to induction therapy with $80 \mathrm{mg}$ subcutaneously and $40 \mathrm{mg}$ at 2 weeks were given placebo, $40 \mathrm{mg}$ every 2 weeks or $40 \mathrm{mg}$ weekly, with $12 \%, 36 \%$ and $41 \%$, respectively, in clinical remission at week $56 .^{486}$ The GAIN trial showed efficacy of adalimumab in patients with active Crohn's disease and loss of response or intolerance to infliximab (secondary infliximab failures). ${ }^{487}$ Data from the EXTEND trial demonstrated adalimumab to be effective in inducing and maintaining endoscopic mucosal healing over the longer term, ${ }^{488}$ and with improved outcomes in those who achieved deep remission. ${ }^{489}$

The signal for the importance of combination therapy with an immunomodulator is not as strong in studies of adalimumab as it is for infliximab. A meta-analysis suggested that combination therapy with an immunomodulator was slightly better than adalimumab monotherapy for induction of remission, but remission rates at 1 year were no different, and there was no reduction in rates of dose escalation compared with monotherapy. ${ }^{490}$ Likewise, the DIAMOND trial comparing adalimumab monotherapy to combination therapy with azathioprine in 176 Japanese Crohn's disease patients naïve to biologics and immunomodulators showed similar remission rates at weeks 26 and 52, ${ }^{491}$ and another study has shown efficacy of monotherapy with adalimumab in maintaining clinical remission for up to 4 years. ${ }^{492}$

A variety of studies on drug levels and immunogenicity have shown a difference between adalimumab monotherapy and combination treatment, with higher trough levels in patients on combination therapy with an immunomodulator, ${ }^{493}$ although clinical benefit was not shown. In the DIAMOND study there was a trend to higher trough drug levels and fewer antibodies against adalimumab with an improvement in endoscopic response at week 26, but not at 1 year. ${ }^{491}$ In another study higher trough levels were associated with endoscopic response

Statement 45 . We suggest that combination therapy of infliximab with methotrexate therapy may be used in Crohn's disease to reduce immunogenicity (GRADE: weak recommendation, moderate-quality evidence. Agreement: $90.5 \%)$. 
and mucosal healing at 6 and 12 months. ${ }^{494}$ In the PANTS 3 -year observational cohort of 1601 Crohn's disease patients treated with infliximab or adalimumab, immunogenicity to adalimumab was present in $11 \%$ at 1 year and $23 \%$ at 3 years, and was associated with non-remission at week $5 .{ }^{481}$ Concomitant immunomodulator therapy reduced immunogenicity (HR 0.34, 95\% CI 0.21 to $0.56, p=0.0001)$. Combination therapy was particularly beneficial in those patients with HLA-DQA1*05 who are more likely to develop antibodies against both adalimumab and infliximab. ${ }^{495}$ A study of UC and Crohn's disease patients losing response to infliximab and adalimumab monotherapy showed anti-drug antibodies present in $61(27 \%)$ of those on adalimumab. Of these, nine patients were given a thiopurine or methotrexate, with six responding with restored clinical response, improved drug levels and loss of antidrug antibodies. ${ }^{496}$ Although it has not been possible to demonstrate the clinical benefits of combination therapy for adalimumab in the way it has been for infliximab combination therapy, there is evidence that concomitant immunomodulator therapy reduces immunogenicity and increases trough levels, which may be clinically beneficial for longer-term adalimumab maintenance therapy.

\subsubsection{Choice of anti-TNF agent in Crohn's disease}

There is little to choose between adalimumab and infliximab in efficacy in luminal Crohn's disease, and practical considerations regarding mode and frequency of administration are the main factors as well as consideration of the relative need for combination therapy with an immunomodulator (see Section 5.2.4.1: Choice of anti-TNF agent). Certolizumab is not licensed for treatment of IBD in the UK and will not be discussed here.

\subsubsection{Vedolizumab}

Vedolizumab is a monoclonal antibody to the $\alpha 4 \beta 7$ integrin and blocks lymphocyte trafficking to the gut by blocking the binding of $\alpha 4 \beta 7$ to the mucosal addressin cell adhesion molecule-1 (MAdCAM-1). It has been demonstrated as effective in inducing remission in the GEMINI-2 trial. ${ }^{497}$ Of 967 patients entering the randomised induction (Cohort 1) and open-label induction (Cohort 2), 461 (47.7\%) had a CDAI fall of 70 points and entered the randomised maintenance phase. Pooled data from the ITT population of the GEMINI-2 and the GEMINI-3 population (a 10-week induction study with dosing at weeks 0 , 2 and 6) presents induction outcomes at weeks 6 and 10, split into 277 TNF-naïve and 490 TNF-failure patients. ${ }^{498}$ Response rates (CDAI fall of 100 points) were better at week 10 on vedolizumab, with TNF-naïve patients responding better $(48.4 \%$ on vedolizumab vs $30.1 \%$ on placebo) compared with TNF-failure ( $39.7 \%$ on vedolizumab vs $22.5 \%$ on placebo). Remission rates at week 10 were also better for TNF-naïve patients $(26.6 \%$ on vedolizumab vs $15.4 \%$ on placebo) compared with TNF-failure ( $21.8 \%$ on vedolizumab vs $11.0 \%$ on placebo). Among induction responders who were re-randomised to placebo or two different

Statement 46. We recommend that in Crohn's disease, vedolizumab can be used in both anti-TNF naïve patients and in those where anti-TNF treatment fails. Choice of treatment in biologics-naïve patients should be individualised (GRADE for induction therapy: strong recommendation, moderatequality evidence; GRADE for maintenance therapy: strong recommendation, high-quality evidence. Agreement: 95.5\%). maintenance vedolizumab dosing strategies, at week 52 clinical remission was achieved in $39 \%$ of patients receiving 8 -weekly infusions and $36.4 \%$ of patients receiving 4 -weekly infusions compared with $21.6 \%$ of patients in the placebo group.

A systematic review has also demonstrated that vedolizumab was superior to placebo in induction and maintenance of remission in IBD and has an acceptable safety profile over the short term. ${ }^{499}$ Vedolizumab responders also appear to have persistence of benefit, with long-term follow-up data from the GEMINI-2 study showing that, of responders at week 6 for whom data were available, $83 \%$ were in remission after 2 years and $89 \%$ after 3 years. ${ }^{500}$ Observational studies have shown consistent findings-for example, a Scottish retrospective study of 153 patients had 1 year steroid-free remission of $28.6 \% .{ }^{501}$ The Swedish SWIBREG study reported 147 patients with active Crohn's disease ( $86 \%$ of whom had previously failed anti-TNF therapy) showed 1 year clinical remission of $54 \% .^{502}$ Recently reported real-world data suggest that higher rates of response with vedolizumab are more likely in patients with Crohn's disease of $\leq 2$ years duration in comparison to those with later stage disease $>2$ years. ${ }^{503}$ This study did not identify an association of response to vedolizumab with disease duration in UC.

In case of slow response to vedolizumab, prednisolone treatment can be maintained initially, or other immunomodulator therapy used or continued for $6-8$ weeks, but as yet there are few data to support this practice.

There are currently no head-to-head comparative trials published of anti-TNF therapy versus anti-integrin therapy. Due to heterogeneity in trial design and patient characteristics, results of network meta-analyses comparing different agents should be treated with some caution, and are no substitute for head-to-head comparisons. ${ }^{504}$ Using propensity score matching, 269 patients with active Crohn's disease in the VICTORY consortium were matched 1:1 with anti-TNF treated patients. At 1 year remission was observed in vedolizumab and anti-TNF treated patients in 38\% and 34\% respectively, HR 1.27 (95\% CI 0.91 to 1.27), steroid-free remission in $26 \%$ and $18 \%$, HR 1.75 (95\% CI 0.90 to 3.43), endoscopic healing in $50 \%$ and 41\% respectively, HR 1.67 (95\% CI 1.13 to 2.47 ). ${ }^{505}$ Side effects of vedolizumab are discussed in the Section 5.2.5 Drug management: vedolizumab and ustekinumab. In biologics-naïve patients, anti-TNF therapy is currently likely to be an initial biologic choice, but there are situations where vedolizumab may be preferred (such as where there is an advantage of gut-specific immunosuppression, or use in older patients where infection and malignancy are a concern), but there are few data to support a clear benefit of anti-integrin therapy in any particular subgroup in Crohn's disease as yet.

\subsubsection{Ustekinumab}

Ustekinumab is an anti-IL12/23 p40 antibody and has been evaluated in the UNITI and IM-UNITI studies in patients with Crohn's disease. UNITI-1 enrolled patients who had prior anti-TNF failure (primary or secondary loss of response or intolerance). Clinical response at week 8 was $37.8 \%$ in those receiving ustekinumab $6 \mathrm{mg} / \mathrm{kg}$ ( $\mathrm{p}<0.001 \mathrm{vs}$ placebo), $33.5 \%$ with $130 \mathrm{mg}$ ( $\mathrm{p}=0.001 \mathrm{vs}$ placebo) and $20.2 \%$ with placebo. ${ }^{506}$ UNITI-2 enrolled patients who had no previous failure with anti-TNF drugs (mostly anti-TNF naïve, but a small number with previous successful use of anti-TNF therapy). Clinical response at week 8 was $57.9 \%$ for $6 \mathrm{mg} / \mathrm{kg}, 47.4 \%$ for $130 \mathrm{mg}$ and $32.1 \%$ for placebo ( $\mathrm{p}<0.001 \mathrm{vs}$ both doses). Responders from both studies at week 8 were randomised to the IM-UNITI maintenance arm. Of these randomised responders, $53.1 \%$ treated 
Statement 47. We recommend that ustekinumab can be used in the induction and maintenance of remission of Crohn's disease, both in anti-TNF naïve patients and in those where anti-TNF treatment fails. No direct comparison data are available with other biological therapies (GRADE: strong recommendation, high-quality evidence. Agreement: 97.7\%).

with $90 \mathrm{mg}$ subcutaneously every 8 weeks ( $\mathrm{p}=0.005$ vs placebo), $48.8 \%$ given $90 \mathrm{mg}$ subcutaneously 12 -weekly ( $\mathrm{p}=0.04 \mathrm{vs}$ placebo) and $35.9 \%$ on placebo were in remission at 44 weeks. In total, $45 \%$ of those randomised to IM-UNITI were anti-TNF refractory. Of these, $41.1 \%$ were in remission at week 44 on ustekinumab $90 \mathrm{mg}$ subcutaneously 8 -weekly compared with $26.2 \%$ on placebo $(p=0.10)$.

Efficacy has been demonstrated in a retrospective observational GETAID study of 122 Crohn's disease patients refractory to anti-TNF drugs. $65 \%$ had clinical benefit within 3 months, and in $68 \%$ of these, benefit was maintained at 12 months. ${ }^{507} \mathrm{~A}$ growing real-world experience confirms the benefit of ustekinumab. ${ }^{508-512}$ Side effects of ustekinumab are discussed in the Section 4.9.5: Vedolizumab and ustekinumab therapy.

\subsubsection{Choice of biological therapy after anti-TNF failure}

To date there are no head-to-head studies comparing ustekinumab and vedolizumab in patients with IBD who have failed anti-TNF therapy, but indirect comparisons suggest no difference in efficacy in this relatively treatment-refractory group. ${ }^{513}$

A consistent theme across multiple clinical trials in Crohn's disease is that response rates are generally lower in patients with a longer disease duration, ${ }^{514} 515$ or who have proven refractory to other therapies. ${ }^{506}$ Given the reduced likelihood of response to therapies in patients who have medically refractory but surgically tractable disease (eg, limited ileocaecal inflammation), surgical approaches should be actively considered to restore quality of life and reduce the risk of complications resulting either from prolonged uncontrolled inflammation or from the use of multiple drug therapies often interspersed with multiple courses of corticosteroid therapy.

\subsubsection{Corticosteroid use and infection risk while on anti-TNF therapy}

The observational Crohn's Therapy, Resource, Evaluation, and Assessment Tool registry (TREAT) was established to compare infliximab safety with conventional non-biological therapy. ${ }^{516}$ Conventional immunomodulator therapies and anti-TNF therapies were associated with an increased risk of infection, including serious and opportunistic infections. Nonetheless the greatest risk of infection, and with it an associated increase in mortality, was seen in patients on corticosteroid therapy (see Section 5.2.1.2: Common Disease Considerations, Infection risk in patients on

Statement 48. We suggest that, where a switch from anti-TNF therapy to a different drug class is required in Crohn's disease, the choice to use vedolizumab or ustekinumab may be made on an individual basis. Factors to be included in the decision-making process should include patient preference, cost, likely adherence, safety data and speed of response to the drug. The potential for surgery as an alternative to further drug therapy should also be considered (GRADE: weak recommendation, very low-quality evidence. Agreement: 97.8\%).
Statement 49. Patients with Crohn's disease treated with a biological therapy in optimal dose who remain corticosteroiddependent (particularly if on triple immunosuppression with immunomodulator therapy) are at significant risk of opportunistic infections. We recommend that alternative medical treatments or surgery should be explored (GRADE: strong recommendation, moderate-quality evidence. Agreement: $97.8 \%)$.

anti-TNF therapy). Requirement for continuous corticosteroid therapy or repeated short courses in patients on biologics suggests that treatment may be failing, and consideration should be given to switching to an alternative (see Section 5.2.4.6: Common Disease Considerations, Secondary loss of response to anti-TNF therapy) or considering other options, including surgery.

\subsubsection{Haematopoietic stem cell transplantation}

Despite the increasing range of drugs available, there are still a number of Crohn's disease patients with severe resistant disease or in whom surgical resection is not appropriate (usually due to extensive disease or incipient short bowel syndrome). For this group of patients, autologous haematopoietic stem cell transplantation (HSTC) has been used. The ASTIC study, an RCT of autologous HSTC published in 2015, set a high bar for its primary end point (of sustained therapy-free clinical, endoscopic and radiological remission at 1 year) and failed to achieve it. ${ }^{517}$ One of the 23 patients undergoing HSTC died and serious adverse events (particularly infection) were common, especially in individuals with perianal Crohn's disease. Nonetheless in this treatment-refractory population there were, among the component parts of the composite primary outcome, suggestions of benefit in some patients and further trial data are needed. ${ }^{518}$

\subsubsection{Leucocyte apheresis}

Although a number of uncontrolled studies have suggested possible efficacy of leucocyte apheresis in IBD, a randomised, double-blind controlled trial in 235 patients with moderate to severely active Crohn's disease showed no benefit of granulocyte/monocyte apheresis over sham apheresis. ${ }^{519}$

\subsection{Proximal jejunal or extensive small bowel disease}

Crohn's disease proximal to the terminal ileum (L4) occurs in $16 \%$ of Crohn's disease patients, and of these about half will have jejunal involvement. ${ }^{520}$ Patients with jejunal involvement are more likely to have stricturing disease, and to require repeated surgery, compared with those who have oesophageal, gastric and/or duodenal disease or those with no disease above the terminal ileum. ${ }^{520}$ In a study of capsule endoscopy, patients with jejunal lesions (in addition to disease elsewhere) were more likely to have relapses over the next 2 years. ${ }^{521}$ In patients having small bowel surgery, those with jejunal disease have a higher recurrence rate, compared with those with ileocaecal disease. ${ }^{522}$ As a consequence of poorer prognosis, patients with jejunal or extensive small bowel disease should be considered for early introduction of biological therapy.

Statement 50. We recommend that leucocyte apheresis is not used for active Crohn's disease due to lack of efficacy (GRADE: strong recommendation, high-quality evidence. Agreement: $100 \%)$. 
Statement 51. Patients with jejunal or extensive small bowel disease have a worse prognosis. We suggest that they may be considered for early use of biological therapy, and should have nutritional assessment and support (GRADE: weak recommendation, very low-quality evidence. Agreement: 100\%).

\subsection{Upper gastrointestinal Crohn's disease}

A multidisciplinary international expert panel (European Panel on the Appropriateness of Crohn's Disease Therapy, EPACT II) published guidance on the management of special situations in Crohn's disease in 2009, including upper gastrointestinal disease. ${ }^{523}$ No discrimination was made between oesophageal, gastric or duodenal disease, and limited evidence was provided in support of recommendations. However, the group split guidance into patients with stenosis and those without, with recommendation that Helicobacter pylori be sought and eradicated in all.

In the absence of stenosis, proton pump inhibitors (PPI) were recommended as first-line therapy, with steroids second-line, and thiopurines and infliximab as third-line. Adalimumab was deemed of uncertain benefit. 5-ASA, certolizumab, surgery and natalizumab were deemed inappropriate.

In the presence of stenosis, balloon dilatation was recommended as first-line therapy, with PPI second-line, and steroids/ thiopurines/surgery as third-line. Infliximab was deemed of uncertain benefit.

Nutritional support is often needed, and may be best delivered by gastrostomy tube for severe or stricturing disease. Exclusive enteral nutrition is beneficial, especially in children. In isolated oesophageal disease, alternative diagnoses should be considered, including gastro-oesophageal reflux disease, sarcoidosis, tuberculosis, disseminated fungal disease, Behçet's disease and malignancy. ${ }^{524}$

\subsubsection{Orofacial granulomatosis}

Orofacial granulomatosis (OFG) is a rare disease with similar microscopic appearances to intestinal Crohn's disease. Granulomatous inflammation is found in about two-thirds of biopsies in OFG but is not needed for a diagnosis. ${ }^{525}$ The precise relationship between OFG and Crohn's disease remains uncertain, with oral Crohn's disease and OFG terminology often overlapping.

Gastroenterologists have been reported to miss around half of oral lesions compatible with oral Crohn's disease when compared with dentist examination. ${ }^{526}$

The threshold for luminal assessment should probably be lower in younger patients, where positive findings are more likely. Sarcoidosis, tuberculosis and chronic infection (particularly dental-associated infection) should be considered in the differential diagnosis. Patients should be referred for assessment to an oral medicine clinic.

The current evidence for OFG management relates to case series only. Topical steroids and tacrolimus are often used, but with limited evidence. An 8-week cinnamon and benzoate-free

Statement 52. We suggest that mild gastroduodenal Crohn's disease may be treated with proton pump inhibitors. We suggest that moderate or severe disease may also require treatment with corticosteroids, and other immunosuppressive or biological therapies as for Crohn's disease elsewhere in the gut (GRADE: weak recommendation, very low-quality evidence. Agreement: $92.7 \%)$.
Statement 53. We suggest that mild oesophageal Crohn's disease may be treated with proton pump inhibitors. Nutritional assessment and support is essential. A short course of corticosteroids may be required if symptoms worsen, but early anti-TNF therapy should be considered for ongoing moderate to severe disease. Oesophageal dilatation should be used for strictures, and surgery is a last resort for disease refractory to all medical therapy (GRADE: weak recommendation, very low-quality evidence. Agreement: 97.6\%).

diet was reported in 25 patients with OFG as first-line therapy with $72 \%$ responding, but with flares on further consumption of previously eliminated cinnamon/benzoate-containing foods. ${ }^{527}$ The mechanism of this effect remains unclear and the long-term continuation of such a diet is challenging. Six weeks of exclusive enteral nutrition (EEN) with Elemental 028 has been used successfully to treat 19 of 22 children with OFG, with 10/10 of isolated OFG cases and 10/12 cases with OFG and Crohn's disease responding. ${ }^{528}$ Objective criteria for improvement were not reported in this study. Such a diet would naturally eliminate cinnamon and benzoate, so may be overly-exclusive in some patients who might have responded to a more targeted elimination.

In a retrospective cohort of 22 non-Crohn's disease OFG patients treated with intralesional triamcinolone injection, $63.6 \%$ of patients had no disease recurrence after a single course of injections. ${ }^{529}$ The mean disease-free period after therapy was 28.9 months (95\% CI 28.7 to 29.1). Response to azathioprine in OFG was significantly greater in those with Crohn's-associated disease. ${ }^{530} \mathrm{~A}$ small case series of 14 OFG patients, half with Crohn's disease, treated with infliximab suggested $71 \%$ shortterm response, but only $33 \%$ were still responding at 2 years. ${ }^{531}$ Two patients with loss of response subsequently responded to adalimumab.

It would seem pragmatic to treat systemic Crohn's disease with standard therapies in the presence of OFG and to add dietary, topical or intralesional approaches if there is no response. Conversely, systemic treatment of isolated OFG should be conserved for disease unresponsive to more limited approaches.

\subsection{Stricturing disease}

For guidance on imaging see the Section 4.1.3.3 Crohn's disease, Diagnostic tests, Investigation of strictures.

\subsubsection{Medical therapy for strictures}

The CREOLE study evaluated 97 Crohn's disease patients with symptomatic small bowel strictures and assessed response to adalimumab treatment. Treatment was successful at week 24 in 64\% of patients, and a prognostic score at baseline was constructed. This included clinical features (use of immunosuppressive drugs at baseline, obstructive symptoms, severity and duration, and MRE features (length of stricture $<12 \mathrm{~cm}$, an intermediate proximal small bowel dilatation $(18-29 \mathrm{~mm})$,

Statement 54 . We suggest that patients presenting with features of orofacial granulomatosis (OFG) and gastrointestinal symptoms, raised inflammatory markers or raised faecal calprotectin should have the gastrointestinal tract investigated for inflammation (GRADE: weak recommendation, very low-quality evidence. Agreement: 100\%). 
Good Practice Recommendation 8. Patients with stricturing small bowel Crohn's disease should have joint medical and surgical assessment to optimise medical therapy and plan requirement for surgical resection or strictureplasty (Agreement: $100 \%$ ).

marked enhancement on delayed T1-weighted sequence and absence of fistula). A higher score was associated with greater likelihood of response to therapy. The authors emphasised the complexity of assessing inflammation and stricturing, which nearly always occur together, and the value of both clinical and MRI features in deciding the value of using drug therapy rather than surgery for small bowel strictures. ${ }^{532}$

Small bowel bacterial overgrowth is common in small bowel Crohn's disease, ${ }^{533}$ particularly in areas above strictures, and responds to broad-spectrum antibiotics ${ }^{534} 535$ (for further detail see the Section on 4.10.1.2.2 Post-surgical management of Crohn's disease: Small intestinal bacterial overgrowth). For nutritional advice for patients with strictures see box 8 .

\subsubsection{Surgical therapy for strictures}

Rates of surgery for Crohn's disease appear to be declining. ${ }^{536537}$ Patient selection and outcomes in stricturing disease can be optimised by careful assessment of fibrotic burden ${ }^{538}$ as well as due consideration of alternatives including endoscopic therapy, ${ }^{539-542}$ and decisions are best taken with joint input from all MDT members. As discussed above, a prognostic score based on MRE features and clinical factors can help in predicting the likely response to anti-TNF therapy. A moderate degree of dilatation proximal to the stricture is associated with greater likelihood of response than either massive dilatation or, rather surprisingly, no dilatation. Having more marked obstructive symptoms, but with a shorter duration, is also associated with success of anti-TNF therapy. ${ }^{532}$

\subsubsection{Strictureplasty}

Strictureplasty, a technique for surgical treatment of small bowel strictures without loss of bowel length, is indicated with single or multiple strictures, impending short gut or previous extensive small bowel resection. The presence of fistulae, fistula-associated abscesses or possible carcinoma are contraindications. The presence of active inflammation at the stricture site does not prevent successful strictureplasty. ${ }^{543}$ Strictureplasty is not associated with increased recurrence or re-operation rates. ${ }^{544-546}$ In fact, there is evidence re-operation rates may be lower at strictureplasty than resection sites, ${ }^{547}$ although a meta-analysis of seven studies (688 patients) from 2006 showed a non-significant trend to lower risk of complications if strictureplasty alone was performed, but a non-significant trend to increased recurrence after strictureplasty and significantly longer recurrence-free survival for those having a bowel resection. ${ }^{548}$ The conventional Heineke-Mikulicz strictureplasty is generally used for strictures up to $10 \mathrm{~cm}$ length, with intermediate length $(10-25 \mathrm{~cm})$ using a modified technique such as the Finney procedure and an enteroenterostomy (Michelassi procedure) for longer strictures. ${ }^{538549}$ The newer techniques are generally as safe and effective as the conventional strictureplasty. ${ }^{550}$

If multiple small bowel strictures can be dealt with by a single resection in a patient with adequate bowel length elsewhere, then this is preferable to avoid a complex multiple strictureplasty procedure, but such decisions have to be individualised, considering the patient's condition at the time of surgery (corticosteroid
Statement 55. We recommend that strictureplasty is an alternative to resection in patients with small bowel Crohn's disease strictures shorter than $10 \mathrm{~cm}$, and is useful where there are multiple strictures or a need to preserve gut length. Longer strictures can be treated using non-standard strictureplasty techniques (GRADE: strong recommendation, very low-quality evidence. Agreement: 97.3\%).

and immunosuppressive drug use, serum albumin, anaemia, nutritional status), potential for postoperative complications from complex surgery and the risk of future malabsorption and malnutrition due to short gut. ${ }^{538550551}$

\subsubsection{Endoscopic therapy for strictures}

Endoscopic balloon dilatation may prevent or delay the need for surgical resection or strictureplasty for Crohn's disease-related intestinal strictures, including anastomotic strictures. A key concern is the long-term outcome, in particular the need for surgical resection. A systematic review identifying 24 published studies reported outcomes from 1163 patients, with overall surgical intervention rates over a median follow-up period of 15-70 months of $27 \% .^{541}$ Surgical intervention rates were higher after dilatation of primary strictures, but this did not reach statistical significance. Stricture length $<4 \mathrm{~cm}$ was associated with a significant reduction in the need for surgical intervention. However, in a different systematic review of largely the same primary papers, 25 studies with data on 1089 undergoing a total of 2664 dilatations estimated the cumulative rate for surgery after 5 years of follow-up as $75 \%$, leading the authors to suggest caution around use of the technique. ${ }^{542}$ However, this figure may overestimate the ultimate requirement of surgery as 5-year follow-up data were only available in 455 patients, who may have remained in follow-up due to a more complicated disease course. The authors did not find any association between stricture subtype and outcomes.

A small study has shown benefit of intralesional injection of corticosteroids at the time of balloon dilatation of Crohn's disease strictures, ${ }^{552}$ but further studies have not shown benefit. $^{553} 554$

\subsection{Non-perianal fistulising Crohn's disease and abscesses} 4.8.1 Intra-abdominal abscesses

4.8.2 Medical therapy

In the first instance, patient optimisation is required when localised perforation in Crohn's disease has led to abscess formation. Surgical intervention is mandatory within the context of free peritonitis and highly likely to be required at some stage in the setting of localised abscess formation. Ideally, surgery takes place following treatment of sepsis and treatment of nutritional deficiencies. Exclusive enteral nutrition can be an effective means of controlling intestinal inflammation without use of corticosteroids.

Some observers suggest that surgery may be avoided by percutaneous radiological drainage of abscesses and then resumption

Good Practice Recommendation 9. If there are multiple strictures close to each other in a segment of bowel and there is adequate remaining healthy bowel, a single resection may be preferable to multiple strictureplasties (Agreement: 100\%). 
Statement 56 . We suggest that balloon dilatation is an appropriate treatment for ileocolonic anastomotic strictures less than $4 \mathrm{~cm}$ in length, without sharp angulation and with non-penetrating disease, although the majority will require repeated dilatation. Endoscopically accessible ileal strictures are also amenable to balloon dilation, but complication rates and recurrence rates are higher (GRADE: weak recommendation, low-quality evidence. Agreement: 94.4\%).

of medical therapy. ${ }^{555}$ The largest case series to date of 51 patients identified retrospectively from review of medical charts from a single centre over a 10 -year period has shown that patients undergoing surgical resection were significantly less likely to develop abscess recurrence than those treated with antibiotics alone or percutaneous drainage over a mean follow-up of 3.75 years $(12 \%$ vs $56 \%) .{ }^{556}$ Nevertheless, non-surgical approaches were successful in preventing subsequent surgery in almost half of the patients treated medically. An almost identical proportion of patients avoided surgery over long-term follow-up in a different series of 32 patients managed with percutaneous drainage for perforating Crohn's disease. ${ }^{557}$ This strategy, if successful in treating sepsis, is likely to lead to lower morbidity and potentially lower stoma rates.

\subsubsection{Enterovaginal and enterovesical fistulae}

Data on the management of non-perianal fistulae, especially from randomised trials, are limited. There are even more limited data on the outcomes of patients treated for specifically entero-gynaecological or enterovesical fistula. Although these fistulae are rare, they are a serious problem to patients with Crohn's disease. A systematic literature review showed that response to medical therapy (fistula closure) was complete in $38.3 \%$ of rectovaginal fistulae and $65.9 \%$ of enterovesical fistulae. ${ }^{558}$ For enterovesical fistulae, risk factors for the need for surgical intervention include sigmoid origin and other complications of Crohn's disease including small bowel obstruction, abscess formation, other fistulation, ureteric obstruction or urinary tract infection. ${ }^{559}$ These authors suggest that enterovesical fistulae should be treated medically initially, with surgery reserved for those with bowel obstruction or abscess formation, non-responders or recurrence after medical therapy. In a series of 47 patients with genital fistulae, antibiotics had no lasting benefit, thiopurines resulted in 13\% complete and 24\% partial response, and anti TNF-alpha therapy gave $17 \%$ complete and 30\% partial response. Surgery was undertaken in a third of patients, but only $22 \%$ had complete response after a first operation and 39\% after reintervention. Overall, fistula closure was only achieved in a third of patients. ${ }^{560}$

These studies highlight that there is still very limited evidence on which to base specific management guidance, but medical therapy alone or in combination with surgery would appear to offer benefit to some patients in the management of enterovaginal and enterovesical fistulae. Patients should be discussed in

Statement 57. We suggest that intra-abdominal abscesses complicating Crohn's disease may be treated initially with intravenous antibiotics and where possible radiological drainage. Surgical drainage may be required but immediate resection should be avoided (GRADE: weak recommendation, very low-quality evidence. Agreement: 91.9\%).
Good Practice Recommendation 10. Following treatment of an abdominal abscess in the setting of non-perianal fistulising Crohn's disease, joint medical and surgical discussion is required, but interval surgical resection is not always necessary (Agreement: 100\%).

multidisciplinary meetings and treatment individualised, considering patients' symptoms and situation.

\subsubsection{Enteroenteric fistulae}

Enteroenteric fistulae are often asymptomatic and expert consensus guidelines have recognised that these do not always require surgery. ${ }^{561}$

\subsubsection{Enterocutaneous fistulae}

Enterocutaneous fistulae usually communicate with segments of active Crohn's inflammation, often in the context of other complications including intra-abdominal abscess and luminal strictures, or with surgical anastomoses (as a manifestation of the poor healing which characterises Crohn's disease). Historically, enterocutaneous fistulae were treated surgically but, with the advent of anti-TNF therapy, conservative approaches can be deployed and may lead to definitive fistula closure in some patients. There are no prospective trial data to guide therapy. Both the fistulae and any associated complications will need to be managed together. For example, in the context of a high output fistula with nutritional deficiency and luminal stricture, a patient will require a period of nutritional and biochemical optimisation, control of sepsis and drainage of collections before definitive surgical management. ${ }^{562563}$

There is little formal evidence on the role of immunosuppressive therapy. If a fistula is associated with active inflammation then medical therapy is worthwhile, but it is unlikely to help a postoperative fistula. ${ }^{564}$ A retrospective series of 48 patients with enterocutaneous fistula from GETAID included 21 postoperative fistulae (within 30 days of surgery, most but not all of which were intestinal resections). ${ }^{565}$ One third had multiple tracts and one quarter had high output. In addition to managing the associated complications, patients were treated with anti-TNF therapy. One third had fistula healing, half of whom relapsed over a median follow-up of 3 years. One third of patients developed an intra-abdominal abscess while on anti-TNF therapy. Surgery was required in 54\% of patients overall. Complexity (multiple tracts) and associated stenosis were associated with reduced rates of healing with anti-TNF therapy and increased need for surgery. Increasing complexity of the fistula is associated with adverse outcomes including mortality. ${ }^{566}$ All patients with enterocutaneous fistulae should be managed by a multidisciplinary team.

\subsubsection{Anti-TNF therapy for non-perianal fistulae}

Higher quality evidence is available for infliximab relative to adalimumab for the use of anti-TNF therapy in the setting of non-perianal fistulising Crohn's disease. The ACCENT II

Statement 58. We recommend that enterovaginal and enterovesical fistulae should be managed jointly with medical control of inflammation and surgical resection (GRADE: strong recommendation, very low-quality evidence. Agreement: 100\%). 
Statement 59. We suggest that low volume enterocutaneous fistulae may be controlled with immunomodulator and biological therapy. High-volume fistulae usually require surgery to achieve symptom control (GRADE: weak recommendation, very low-quality evidence. Agreement: 100\%).

trial included 25 women with a total of 27 draining rectovaginal fistulae at baseline. ${ }^{567}$ Closure at week 14 after open label infliximab induction therapy was achieved for $45 \%$ of fistulae. Randomisation to infliximab during maintenance therapy was associated with a longer duration of fistula closure compared with placebo therapy (median 46 weeks vs 33 weeks). Outcomes for patients in ACCENT II with enterocutaneous fistulae have not been reported separately, but these patients represented less than $10 \%$ of the total cohort. Adalimumab has never been subjected to a randomised control trial of efficacy in fistulising Crohn's disease.

\subsection{Perianal Crohn's disease}

\subsubsection{Assessment of perianal disease}

Multidisciplinary team working in perianal Crohn's disease is widely regarded as best practice according to expert consensus. $^{561}$

Pelvic MRI has a well-recognised role in defining the anatomy of perianal fistulae as an adjunct to examination under anaesthesia (EUA). Initial reports of sensitivity compared with EUA suggested rates of $85-89 \% .{ }^{568-570}$ MRI may also pick up a significant burden of non-fistulising perianal disease that would otherwise be missed clinically. ${ }^{570} 571$ Endoanal ultrasound has also shown high sensitivity, although use is limited in patients with anal or other luminal stenoses. ${ }^{569} 570$ Interpretation of fistula imaging (MRI or endoanal ultrasound) is dependent on operator expertise.

An experienced colorectal surgeon should perform EUA to assess perianal disease and identify the anatomy of the fistula tracts, as specialist expertise and knowledge are likely to improve identification of all tracts. ${ }^{569} 572573$ In a comparison with endoscopic ultrasound and MRI, an experienced operator correctly classified 91\% of patients' perianal fistulae at EUA (95\% CI 75\% to 98\%). ${ }^{569}$ However, a combination of two tests from MRI, endoanal ultrasound and EUA is recommended to give the optimal assessment. ${ }^{574}$ Specifically, unless directed by the results of MRI or endoanal ultrasound, then small abscesses or collections may be missed, particularly if there is significant induration or a supralevator collection. ${ }^{574}$ EUA is more sensitive than MRI in assessment of anal ulceration or anal canal stenosis. $^{571}$

Assessment of rectal mucosa at the time of EUA is important. ${ }^{572} 575$ Largely observational data, but with a large effect size, from the pre-biologics era show that outcomes are worse for perianal fistulising disease associated with rectal inflammation. A study from St Mark's of perianal fistulising Crohn's disease patients diagnosed in 1993-4 showed that 29\% of those with rectal involvement had proctectomy compared with $4 \%$ of those with no rectal involvement. ${ }^{576}$

Statement 60 . We suggest that anti-TNF therapy may be used to control inflammation or maintain remission in the setting of non-perianal fistulising Crohn's disease (GRADE: weak recommendation, low-quality evidence. Agreement: 94.6\%).
Statement 61. We recommend that anti-TNF therapy should only be started after abscesses have been treated with antibiotics and, where possible, drainage (GRADE: strong recommendation, very low-quality evidence. Agreement: 94.9\%).

\subsubsection{Seton insertion}

EUA permits drainage of abscesses and placement of setons, with evidence that placing setons prior to starting anti-TNF therapy improves rates of healing and reduces recurrence rates. ${ }^{577-579} \mathrm{~A}$ recent meta-analysis confirmed the benefits of combined surgical and medical therapy in improving healing and makes recurrent abscess less likely. ${ }^{580}$ The exception to routine insertion of setons would be patients with rectovaginal fistulae in the absence of an abscess or collection. In this situation, setons may make faecal discharge per vagina worse.

Evidence for the timing of seton removal is unclear. Early studies removed setons at or shortly after the second infliximab infusion. 567577579 More recent studies suggest later removal, either after completing infliximab induction therapy ${ }^{581}$ or after at least five infusions. ${ }^{52}$ Setons need to be removed to permit complete healing of fistula tracks, but if active inflammation is ongoing, then seton removal may result in recurrent abscess. The optimal timing and assessment method remain unclear, and some patients may require long-term setons to avoid or delay proctectomy. Further trials are needed to better understand the optimal management of perianal fistulae in Crohn's disease. ${ }^{583} 584$

\subsubsection{Anti-TNF therapy post-surgery for complex perianal fistulae 4.9.3.1 Infliximab for perianal fistulising disease}

Higher level evidence is available for infliximab relative to adalimumab for complex perianal fistulae following initial surgical management, such as draining abscesses and seton placement. Use of infliximab has been shown to be effective for the closure of perianal fistulae. In a study of 94 patients with Crohn's disease and abdominal or perianal fistulae, induction therapy with $5 \mathrm{mg} /$ $\mathrm{kg}$ or $10 \mathrm{mg} / \mathrm{kg}$ was associated with complete fistula closure rates of $55 \%$ and $38 \%$ compared with $13 \%$ placebo. ${ }^{585}$ In the subsequent ACCENT II trial, this induction response was confirmed in an open-label phase, with fistula closure in $69 \%$ of patients at 14 weeks. Subsequent randomisation to infliximab was associated with a significantly longer median time to loss of response compared with patients randomised to placebo ( $>40$ weeks vs 14 weeks), with $36 \%$ of patients completing this maintenance phase showing a complete absence of draining fistulae after 54 weeks of infliximab treatment compared with $19 \%$ of placebo patients $(p=0.009) .{ }^{567}$

Higher infliximab doses may be beneficial for perianal fistulising disease, with target levels $>10 \mu \mathrm{g} / \mathrm{mL}$ associated with better response. ${ }^{586}$

\subsubsection{Adalimumab for perianal fistulising disease}

Fistula closure or improvement has not been the primary outcome of any prospective randomised trials of adalimumab. The CHARM trial showed increased efficacy compared with placebo for closure of abdominal or perianal fistulae as a

Good Practice Recommendation 11. Multidisciplinary decision-making should be the standard of care for patients with perianal Crohn's disease (Agreement: 97.4\%). 
Statement 62. We recommend that pelvic MRI is used as an important adjunct to clinical assessment and examination under anaesthesia (by an experienced surgeon) in evaluation of fistulising perianal Crohn's disease. Depending on local availability and expertise, endoanal ultrasound may also be used (GRADE: strong recommendation, high-quality evidence. Agreement: 100\%).

secondary end-point, but outcomes for fistula subtypes have not been reported. At baseline there were 117 patients with draining fistulae. All patients received open-label induction therapy with adalimumab and were then randomised to maintenance therapy with adalimumab or placebo. Complete fistula closure at week 56 was seen in 33\% of subjects on adalimumab versus 13\% on placebo $(\mathrm{p}=0.016) .{ }^{486}$ Of all those with healed fistulae at week 56 (including those on placebo), 90\% (28/31) maintained healing after a year of open-label adalimumab. ${ }^{587}$

A randomised controlled trial in which 76 patients with active perianal fistulising disease received open-label therapy with 24 weeks adalimumab in combination with either ciprofloxacin $500 \mathrm{mg}$ BD for 12 weeks or placebo found that, after 12 weeks, the primary end-point of $50 \%$ reduction in draining fistulae was achieved in $71 \%$ in the combination adalimumab/ ciprofloxacin group against $47 \%$ in the adalimumab/placebo group $(\mathrm{p}=0.047)$. $^{588}$ However, by week 24 (ie, 12 weeks after cessation of antibiotic therapy) the difference between the two groups was no longer significant. This suggests the addition of an antibiotic may speed healing but not affect the final outcome. Further evidence is required before this can be recommended as routine practice.

\subsubsection{Combined surgical treatment with anti-TNF therapy}

None of these studies included initial surgical interventions for the management of fistula tracts. No randomised controlled trial has compared these treatments directly or attempted to evaluate the additional benefit of combination therapy compared with treatment with either surgery or anti-TNF alone. Nevertheless, comparison between these approaches has been the subject of several retrospective studies which have recently been systematically reviewed. Although significant heterogeneity between individual studies limits interpretation, there is a consistent trend towards improved outcomes with combined surgical and anti-TNF treatment. ${ }^{580} 583$ Careful preparation of the fistula track with curettage to destroy all epithelial tissue and ligation of the internal opening as standard treatment in both active and control arms of one trial of mesenchymal stem cells achieved a 'placebo' (surgical preparation only with no active medical treatment) success rate of $34 \%$ at week $24 .{ }^{589}$ It is highly likely that multiple treatment modalities will be needed to control perianal fistulae in Crohn's disease. ${ }^{590}$

\subsubsection{Vedolizumab and ustekinumab therapy}

Post hoc analysis of 57 patients with fistulae (site not specified) in the GEMINI 2 study showed higher rates of closure of draining

Statement 63. We recommend that examination under anaesthesia should include an assessment of the rectal mucosa as the presence of proctitis is associated with lower rates of fistula healing in perianal Crohn's disease (GRADE: strong recommendation, high-quality evidence. Agreement: 100\%).
Statement 64 . We recommend that setons should be placed to prevent re-accumulation of perianal sepsis in fistulising Crohn's disease (GRADE: strong recommendation, moderate-quality evidence). The optimal timing of seton removal is uncertain (Agreement: 97.1\%).

fistulae at 1 year: $41.2 \%$ on 8 - weekly vedolizumab $(p=0.03$ vs placebo) and $22.7 \%$ on 4 - weekly vedolizumab (not significant vs placebo). ${ }^{497}$

Data for outcomes for those patients with fistulae at baseline in the CERTIFI phase 2o and UNITI phase 3 studies of ustekinumab have been reported in abstract form, showing a non-significant trend towards improved fistula healing in patients randomised to ustekinumab compared with placebo. ${ }^{591}$ Additional data suggesting healing with ustekinumab come from relatively small case series of TNF refractory patients. 507508592 Further controlled trial data are needed to confirm the role of ustekinumab in perianal fistula healing.

\subsubsection{Surgical treatment of fistulae}

In a survey of British surgeons, commonly performed definitive procedures in the context of perianal Crohn's disease were removal of draining seton only (70.7\%), fistulotomy (57.1\%), and less commonly advancement flap (38.9\%), fistula plug (36.4\%) and the ligation of intersphincteric track (LIFT) procedure $(31.8 \%) .{ }^{593}$ The reported efficacy of curative surgical options within the context of perianal Crohn's disease is variable. Advancement flaps and the LIFT technique are effective in selective patients with perianal Crohn's disease, with healing observed in two-thirds of patients at 1 year in a small prospective study. ${ }^{594}$ There is, however, progressive failure over time, related to both treatment failure and recurrent disease. ${ }^{595}$ Video-assisted anal fistula treatment (VAAFT) combined with advancement flap has been reported in complex fistulising Crohn's disease with an $82 \%$ success rate at 9 months. ${ }^{596}$ Systematic review has demonstrated fistula plugs may be effective in 55\% of Crohn's disease-related fistulae, ${ }^{597}$ although variation in reported success is wide. A prospective randomised control trial of fibrin glue in 36 patients with Crohn's disease perianal fistulae showed effective therapy in $38 \%$ after 8 weeks. ${ }^{598}$ In comparison, just $16 \%$ of those under observation only after removal of seton achieved remission $(p=0.04)$. The benefit was greater in patients with simple fistulae. Administration of expanded adipose-derived stem cells in addition to fibrin glue appears more effective than fibrin glue alone. ${ }^{599}$ The long-term efficacy of fibrin glue, collagen plugs and paste is not clear.

\subsubsection{Allogeneic adipose-derived stem cell therapy for perianal fistulae}

Expanded allogeneic adipose-derived stem cells are simple to administer and safe. ${ }^{600}$ In a recent phase III study, 212 patients with treatment-refractory complex perianal fistulising Crohn's

Statement 65 . We recommend that infliximab is used as the first-line biological therapy for complex perianal fistulae, and should be started as soon as adequate drainage of sepsis is achieved (GRADE: strong recommendation, high-quality evidence. Agreement: 100\%). 
Good Practice Recommendation 12. Surgical options for perianal Crohn's disease fistulae (advancement flap, LIFT, infill procedures) should only be offered in selected patients after counselling as long-term results are poor, particularly for those with complex disease and ongoing disease activity. (Agreement: $97.1 \%)$.

disease were randomly assigned to a single intralesional injection of 120 million Cx601 cells (darvadstrocel) or placebo. ${ }^{589}$ Both active and control groups had an initial EUA with curettage of fistula tracts, and insertion of setons if needed, followed at least 2 weeks later by a further EUA with ligation of the internal fistula opening and injection of the trial treatment into the tissue adjacent to all fistula tracts. Week 24 complete remission rate was $50 \%$ for active treatment versus $34 \%$ for placebo $(p=0.024)$. The high placebo response rate likely reflects the extensive surgical interventions received by the placebo group including curettage of the fistula tract and closure of the internal opening. Of note, failure of anti-TNF therapy was not a requirement for study entry, but rather patients had to have failed at least one of antibiotics, immunomodulator therapy or anti-TNF therapy. In fact, $79 \%$ of patients in this trial had been on an anti-TNF in the previous 6 months and $61 \%$ were on anti-TNF therapy at the point of randomisation. One-year outcome data demonstrate combined remission (closure of all treated external openings that were draining at baseline, and absence of collections $>2 \mathrm{~cm}$ on MRI) of $56.3 \%$ in the darvadstrocel group, versus $35.6 \%$ in the placebo group $(p=0.021)$. Clinical remission at 1 year was $59.2 \%$ and $41.6 \%$ respectively $(p=0.013) .{ }^{601}$ Other groups have reported promising data with alternative preparations of allogenic stem cells, reviewed recently. ${ }^{602}$ Given the complexity of appropriate patient selection, management of luminal disease, surgical technique, selection of adjunct therapies and choice of stem cell preparation, patients offered these therapies should be closely monitored as part of a clinical trial or registry.

\subsubsection{Defunctioning stoma formation}

Creation of a defunctioning stoma has long been recognised to offer rapid improvement of perianal Crohn's disease, even in complex cases. The mechanism likely relates to diversion of the faecal stream, which of itself seems to contain factors that promote rectal inflammation. ${ }^{603}$ Evidence is limited to expert opinion, supported by several case series. ${ }^{604-607}$ Although many of these series originate from a pre-biologics era, more recent data suggest that rates of successful stoma reversal after ileostomy for complex perianal Crohn's disease are low. ${ }^{608} 609$ A systematic review showed that faecal diversion improves symptoms in approximately two-thirds of patients, but bowel restoration is only successful in $17 \% .{ }^{610}$ Rates of successful reversal may be particularly low in those with rectal involvement. ${ }^{610}$ It should be noted that, after initial improvement, recurrence of inflammation can occur in the pelvis and defunctioned segment of bowel, and may present non-specifically with weight loss, raised inflammatory markers and fevers. For severe refractory disease, proctectomy is an effective treatment, but considered by expert consensus to be a last resort. ${ }^{607}$

\subsection{Post-surgical management of Crohn's disease}

\subsubsection{Disease recurrence following ileocolonic resection}

In a study of factors predictive of recurrent disease after ileocaecal resection there was a symptomatic recurrence rate of $20 \%$ at 1 year and $47 \%$ at 5 years. There was a rapid
Statement 66 . We recommend that faecal stream diversion can be used in patients with severe perianal Crohn's disease refractory to medical therapy. Patients should be counselled that rates of subsequent successful reversal are low and proctectomy may ultimately be required (GRADE: strong recommendation, low-quality evidence. Agreement: 100\%).

development of endoscopic lesions in the neoterminal ileum (73\% at 1 year). ${ }^{359}$ Risk factors for disease recurrence are discussed in the section below on medical prophylaxis (see Section 4.10.3: Reassessment and medical prophylaxis following ileocolonic resection).

\subsubsection{Investigation for symptomatic recurrence following ileocolonic resection}

After resection for Crohn's disease of all inflamed tissue, the cumulative rate of symptomatic recurrence at 3 years is approximately 50\%. ${ }^{359}$ Patients presenting with new symptoms of pain or diarrhoea after resection should be evaluated to confirm whether recurrent disease is the cause, as the differential diagnosis includes bile salt malabsorption and irritable bowel syndrome. Although ileocolonoscopy is the gold standard assessment to determine postoperative recurrence, there are times when it is not appropriate or technically possible and cross-sectional imaging may be needed. There is good agreement between assessment by MR enteroclysis and endoscopy: mean observer agreement was seen in $77.8 \%$ (kappa 0.67 ) in the diagnosis of postoperative recurrence. ${ }^{611}$ In experienced hands, small bowel ultrasound is predictive of endoscopic findings, ${ }^{612-614}$ with good correlation to the Rutgeerts score. ${ }^{615}$ While CT has also been shown to be effective in identifying postoperative recurrence, ${ }^{616} 617$ it should be avoided where possible to limit radiation exposure.

Faecal calprotectin and lactoferrin in postoperative Crohn's disease patients correlate well with clinical disease activity as measured by the Harvey Bradshaw Index, whereas the correlation with C-reactive protein was weaker. ${ }^{618}$ There was, however, low sensitivity and specificity of the stool tests, particularly in those with mild or moderate clinical symptoms. In addition, this study did not show that faecal calprotectin was predictive of endoscopic (rather than clinical) recurrence. In the POCER trial, faecal calprotectin correlated with endoscopic recurrence, with a level $>100 \mu \mathrm{g} / \mathrm{g}$ stool indicating endoscopic recurrence with a sensitivity of $89 \%$ and negative predictive value of $91 \% .{ }^{619} \mathrm{~A}$ further multicentre observational cohort study of 86 asymptomatic Crohn's disease patients following ileocaecal resection also identified a cut-off of faecal calprotectin of $100 \mu \mathrm{g} / \mathrm{g}$ as best to discriminate between endoscopic recurrence versus remission. ${ }^{620}$ In this study the sensitivity, specificity, negative predictive value, positive predictive value and overall accuracy at $100 \mu \mathrm{g} / \mathrm{g}$ were 95\%, 54\%, 69\%, 93\% and 77\%, respectively. The TOPPIC

Statement 67. We suggest that, in the event of symptomatic recurrence following ileocolonic resection for Crohn's disease, an assessment of mucosal inflammation may be performed with ileocolonoscopy. Faecal calprotectin and/or cross-sectional imaging may be used if ileocolonoscopy is not possible or acceptable, but may not be sensitive enough to detect localised inflammation (GRADE: weak recommendation, low-quality evidence. Agreement: 97.4\%). 
Statement 68. We recommend that, following ileal resection, Crohn's disease patients with diarrhoea suggestive of bile acid malabsorption should be given a therapeutic trial of a bile acid sequestrant such as colestyramine or colesevelam (GRADE: strong recommendation, moderate-quality evidence). A SeHCAT study can be considered for failed response to therapy or if the diagnosis is unclear (Agreement: 97.2\%).

trial also showed association of faecal calprotectin with clinical recurrence. ${ }^{621}$ TOPPIC also demonstrated the power of faecal calprotectin as a time-dependent variable, in that a $100 \mu \mathrm{g} / \mathrm{g}$ rise in calprotectin led to an $18 \%$ increase in the HR for clinical recurrence. It is suggested therefore that the use of faecal calprotectin is most helpful when there is a comparison value for the same patient when in known remission. A rise in the background level in the context of clinical symptoms should trigger further investigation and treatment.

\subsubsection{Non-inflammatory causes of diarrhoea after ileocolonic resection}

Gastrointestinal symptoms in patients with previous surgery for Crohn's disease are not necessarily related to recurrent inflammation. Conversely, minor anastomotic Crohn's disease recurrence is often asymptomatic. Other diagnoses should be considered.

4.10.1.2.1 Bile salt malabsorption

Bile acid diarrhoea due to malabsorption is common following ileal resection, occurring in more than $80 \%$ of patients. $^{622-625}$ A therapeutic trial of bile acid sequestrants is therefore appropriate, particularly if faecal calprotectin is not significantly raised. A ${ }^{75}$ Se-HCAT (SeHCAT) scan should only be requested when there is uncertainty, as it is often abnormal after ileal resection or with ileal inflammation, and an abnormal scan does not prove that symptoms are due to bile salt malabsorption. Colestyramine is effective ${ }^{623}{ }^{626}$ but may be unpalatable, and other agents such as colestipol or colesevelam, which are more expensive, can be used as alternatives for those who do not tolerate it. ${ }^{622}$ Bile acid sequestrants need to be stopped during the SeHCAT test. Loperamide can also be used. ${ }^{627}$ There is increasing interest in bile acid malabsorption as a cause of functional diarrhoea. ${ }^{628}$ Serum markers of bile acid diarrhoea, such as reduced fibroblast growth factor-19 levels ${ }^{629} 630$ and raised 7-alpha-hydroxycholestenone (C4), ${ }^{631}$ are not yet widely available; both tests will be abnormal in ileal resection, and also with ileal inflammation.

4.10.1.2.2 Small intestinal bacterial overgrowth

Small intestinal bacterial overgrowth occurs more commonly after Crohn's disease resection, ${ }^{533}$ and can mimic Crohn's disease in causing symptoms of bloating, diarrhoea, nausea or vomiting, weight loss or malnutrition. ${ }^{632}$ The prevalence in one study was 30\% using a lactulose breath test, measuring hydrogen and methane. ${ }^{633}$ Bacterial overgrowth is more common if there are blind loops, dysmotility, diverticulae or strictures. The

Good Practice Recommendation 13. Patients with recurrent symptoms following resection for Crohn's disease, who have no evidence of active inflammation, should have consideration of other diagnoses including bile salt malabsorption, bacterial overgrowth, functional bowel disorders, adhesions, fibrostenotic or anastomotic strictures (Agreement: 97.3\%). gold standard test for small intestinal bacterial overgrowth is jejunal aspirate with quantitative culture for aerobic and anaerobic organisms. This test is invasive and usually not available. Glucose or lactose hydrogen breath tests are more often used. Measurement of methane as well as hydrogen is recommended to increase sensitivity, but there is a lack of standardisation and poor sensitivity and specificity for these tests. ${ }^{634}$ Empirical treatment is recommended if the diagnosis is likely, with broad-spectrum antibiotics such as metronidazole, ciprofloxacin ${ }^{534}$ or rifaximin. ${ }^{635}$ Recurrent courses of treatment may be required. 4.10.1.2.3 Other causes of recurrent symptoms following ileocolonic surgery

Other co-existing conditions that can cause diagnostic confusion include irritable bowel syndrome, ${ }^{636}$ coeliac disease and short gut syndrome in those with extensive small bowel resection. Lymphoma and malignancy must also be considered. Postoperative complications should also be considered, including anastomotic strictures, collections and port-site hernia (after laparoscopic surgery).

\subsubsection{Smoking cessation following surgery}

The best described environmental factor affecting the outcome of IBD is cigarette smoking, which has a negative impact on the clinical course of Crohn's disease. It has been shown that cigarette smokers have quicker and more severe postoperative relapse in Crohn's disease. ${ }^{621637}$ Thus, all efforts should be made to help patients quit smoking after surgery by offering counselling, pharmacotherapy or nicotine replacement therapy (see Section 5.8.1: Smoking and Crohn's disease).

\subsubsection{Reassessment and medical prophylaxis following ileocolonic} resection

A summary flowchart of postoperative medical prophylaxis following ileocolonic resection for Crohn's disease is shown in figure 3 .

\subsubsection{Risk factors for disease recurrence after ileocolonic resection}

Surgery for the majority of patients with Crohn's disease is not curative, with high rates of disease recurrence by 1 year. ${ }^{638}$ Endoscopic recurrence predicts future clinical relapse. ${ }^{359639} \mathrm{By}$ 6 months, endoscopic recurrence is apparent in between $39 \%$ and $84 \%$ of patients. ${ }^{640-642}$ A meta-analysis of the placebo groups of postoperative maintenance trials showed an endoscopic recurrence rate of $58 \%(95 \%$ CI $51 \%$ to $65 \%)$ at a median 1 year after surgery. ${ }^{643}$

Clinical factors predictive of symptomatic recurrence in the Rutgeerts study were preoperative disease activity, indication for surgery (fistulae and abscess being associated with more recurrence than strictures) and number of previous resections. ${ }^{359}$ Severity of recurrent mucosal lesions in the neoterminal ileum at colonoscopy after resection was the main predictor of subsequent symptomatic recurrence on multivariable analysis. Preoperative disease activity was the main clinical predictive factor. Smoking was not evaluated in this study but is the most important risk factor, with meta-analysis showing an increased

Statement 69. We recommend that all patients smoking after intestinal resection for Crohn's disease should be actively encouraged to stop (GRADE: strong recommendation, moderatequality evidence. Agreement: 100\%). 
Statement 70 . We suggest that, following ileocolonic resection for Crohn's disease, ileocolonoscopy may be performed at 6 months to assess the neoterminal ileum in order to consider treatment escalation if mucosal inflammation (Rutgeerts i2 or above) (GRADE: weak recommendation, low-quality evidence). If the anastomosis is not within reach of endoscopic examination, then cross-sectional imaging with MR enterogram may be performed (Agreement: 89.2\%).

risk of endoscopic recurrence by 2.5 -fold and clinical recurrence 2 -fold. ${ }^{637}$ Other risk factors for postoperative recurrence include prior resection, penetrating disease, perianal disease, extensive bowel disease $(>50 \mathrm{~cm})$, granulomas in the resection specimen and myenteric plexitis in the proximal resection margin. ${ }^{643-646}$

\subsubsection{Evidence of benefit from medical prophylaxis to prevent postoperative recurrence}

Postoperative prevention trials have been challenging in this area as clinical recurrence is less common than endoscopic recurrence, and commonly used end-points for clinical relapse such as the CDAI score are not validated in the postoperative setting. It has been much easier to demonstrate reduction in endoscopic recurrence rates, and endoscopic lesion severity does correlate with clinical recurrence risk. ${ }^{359} 647$

4.10.3.2.1 Thiopurine therapy

For those who are not able to stop smoking, the TOPPIC trial provided evidence that thiopurines may improve their chance of maintaining remission after surgery. This study did not, however, confirm a wider role for thiopurines in the postoperative context, with no overall difference in either clinical or endoscopic recurrence at 3 years (although a post-hoc analysis showed that complete endoscopic healing (Rutgeerts score i0) was more likely in the mercaptopurine group). ${ }^{621}$ A Cochrane review of previous trials showed low quality evidence for benefit of thiopurines in comparison to placebo. ${ }^{648}$

4.10.3.2.2 Anti-TNF therapy

Early studies had shown benefit for infliximab in preventing postoperative recurrence, but in general it has been easier to demonstrate prevention of endoscopic recurrence at 1 year ${ }^{649} 650$ or 2 years, ${ }^{651}$ as clinical relapse rates in these studies were lower and differences non-significant. The Yoshida ${ }^{650}$ study had follow-up at 3 years and there was a significantly higher remission rate of $93.3 \%$ on infliximab compared with $56.3 \%$ on no treatment. A study by Sorrentino et $a l^{652}$ evaluated those who remained in clinical and endoscopic remission on infliximab at 2 years post-surgery, at which time the treatment was stopped. Ten of the 12 patients had endoscopic relapse at 4 months and all achieved healing on retreatment with lower doses of infliximab (3 mg/kg 8-weekly). A large double-blind placebo-controlled trial of postoperative infliximab in 297 patients did not demonstrate significant reduction in clinical relapse at 76 weeks $(12.9 \%$ on

Statement 71. We suggest that Crohn's disease patients with significant risk factors for disease recurrence following ileocolonic resection (particularly smoking) or with recurrent disease at 6 months post-surgery colonoscopy may be started on thiopurines (GRADE: weak recommendation, low-quality evidence) or anti-TNF therapy (GRADE: weak recommendation, low-quality evidence. Agreement: 94.4\%).
Statement 72 . We recommend that mesalazine should not be given to prevent recurrence after ileocolonic Crohn's disease resection (GRADE: strong recommendation, moderate-quality evidence. Agreement: 97.2\%).

infliximab) compared with placebo (20\%), but did show reduction in endoscopic recurrence $(30.6 \%$ vs $60 \%)$. Clinical relapse rates were low despite patients having to have a risk factor for recurrence (previous surgery, resection for penetrating disease, recent perianal fistulae or current smoking). ${ }^{653}$ For patients given anti-TNF prophylaxis postoperatively, the use of multiple anti-TNF drugs in the past makes relapse much more likely. ${ }^{654}$ A randomised three-arm study compared postoperative adalimumab against azathioprine and 5-ASA with a 2-year follow-up. There was significantly reduced endoscopic recurrence for those on adalimumab (adalimumab 6.3\%, azathioprine 64.7\%, 5-ASA $83.3 \%)$ and significantly reduced clinical recurrence $(12.5 \%$, $64.7 \%$ and $50 \%$, respectively). ${ }^{655}$

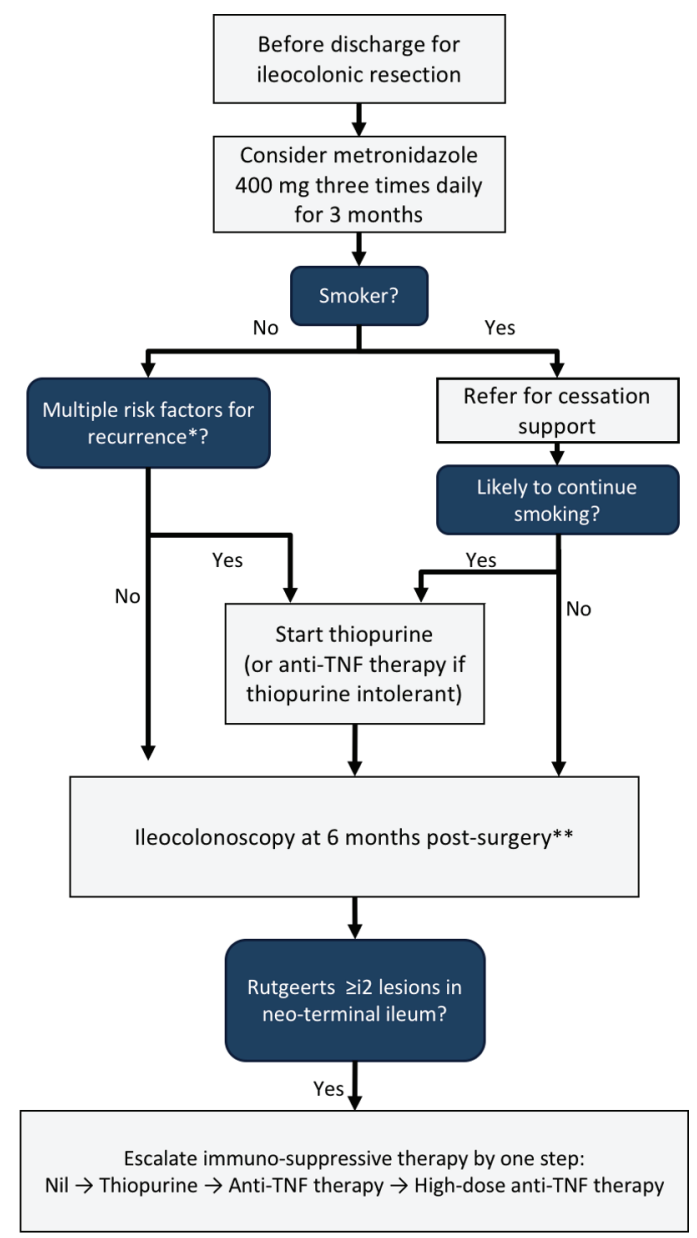

* Risk Factors for recurrence (two or more of):

- Smoking

- Penetrating disease

- Multiple resection

- Perianal fistulae

- Extensive small bowel disease

- Residual active disease

- Granulomas or myenteric plexitis

** If not possible consider cross-sectional imaging or faecal calprotectin as alternative

Figure 3 Medical prophylaxis after ileocolonic resection for Crohn's disease. 
4.10.3.2.3 Postoperative colonoscopy at 6 months

The POCER trial was a pragmatic randomised trial that compared an active care model using endoscopic assessment at 6 months postoperatively with standard care (no colonoscopy at 6 months) ${ }^{640}$ All patients entering the trial received metronidazole for 3 months postoperatively, and those at high risk of recurrence received thiopurines (or adalimumab if intolerant). In the active care group, treatment was stepped up if 6-month endoscopic recurrence was documented: to thiopurine, fortnightly adalimumab with thiopurine, or weekly adalimumab. At 18 months endoscopic recurrence was $49 \%$ in the active group versus $67 \%$ in the standard care group $(p=0.03)$ and clinical recurrence was $27 \%$ and $40 \%$, respectively $(\mathrm{p}=0.08)$.

4.10.3.2.4 Antibiotic therapy

Antibiotic therapy can maintain remission for at least 3 months after surgery. ${ }^{656657}$ Metronidazole is the most widely used, ${ }^{658}$ but is poorly tolerated $(23 \%$ dropped out during the 3 months trial treatment on metronidazole $20 \mathrm{mg} / \mathrm{kg}$ daily), and causes neuropathic complications which relate to total dose and generally prohibit longer use. Ornidazole is also effective but still limited by significant side effects $(32 \%$ dropped out during the 1 year trial treatment). ${ }^{659}$ In a small pilot study, ciprofloxacin did not show much benefit over placebo. ${ }^{660}$ In view of its gut specificity and reduced side effect profile, there is interest in using rifaximin in this context. There is a study of rifaximin for maintenance therapy over 3 months after medically-induced remission, ${ }^{661}$ but trials are awaited in the postoperative setting.

4.10.3.2.5 Other treatment

Evidence regarding 5-ASA is conflicting, with small studies showing no significant benefit. Meta-analysis has shown a modest reduction in clinical recurrence and severe endoscopic recurrence, ${ }^{662}$ but less effectiveness than other therapies. ${ }^{663}$ Likewise, steroids have significant toxicity and are ineffective in maintaining postoperative remission. ${ }^{656}$ As yet there are not enough data to specifically recommend vedolizumab or ustekinumab in this context.

\section{COMMON DISEASE CONSIDERATIONS}

\subsection{Infectious diseases and IBD: differential and concurrent diagnoses}

\subsubsection{Tuberculosis}

Differentiating between intestinal tuberculosis (TB) and Crohn's disease may be challenging in those who have lived in endemic areas as clinical features may be similar. Features suggestive of a diagnosis of intestinal TB include night sweats, concomitant pulmonary tuberculosis, positive tuberculin skin test, antibodies to TB, abdominal lymphadenopathy, ascites, transverse ulcers and a patulous ileocecal valve. ${ }^{664-666}$ Haematochesia, involvement of the sigmoid colon, skip lesions and aphthous ulceration are more common in Crohn's disease, whereas weight loss may be predictive of TB. ${ }^{665}$ Extraintestinal manifestations are more commonly associated with Crohn's disease. ${ }^{666}$ A recent study from South Korea used 40 Crohn's patients and 40 with intestinal TB to develop a predictive model using colonoscopic, laboratory and radiological criteria. Features suggesting Crohn's at colonoscopy were anorectal lesions, longitudinal ulcers, aphthous ulcers and cobblestoning. Colonoscopic features suggesting TB were fewer than four segments of colon involved, patulous ileocaecal valve, transverse ulcers and scars or post-inflammatory polyps. Positive ASCA serology and proximal small bowel disease suggested Crohn's, and a positive quantiferon gold test and typical pulmonary lesions suggested TB. A score derived from these features
Statement 73. We recommend that the differential diagnosis of tuberculosis should be considered in patients with suspected ileocaecal Crohn 's disease, particularly in patients born in or who have lived for extended periods in endemic areas or have other risk factors for infection (GRADE: strong recommendation, very low-quality evidence. Agreement: 97.8\%).

was able to make an accurate diagnosis in $96.3 \%$ of patients. ${ }^{667}$ TB PCR performed on intestinal biopsies may be helpful for discrimination between the diseases. ${ }^{668}$

\subsubsection{Enteric infections associated with IBD}

The risk of enteric infections is higher in IBD than controls. ${ }^{669}$ A UK study showed that $10.5 \%$ of IBD relapses were associated with enteric infection (half were Clostridium difficile) ${ }^{670} \mathrm{~A}$ more recent retrospective study in over 9000 Mayo Clinic IBD patients from whom stools were collected showed a low rate of bacterial infection of less than 3\% by culture and PCR (excluding C. difficile infections), with no adverse impact on disease course (higher proportions of stool positive patients remained in remission over the next year compared with those with a non-infective disease flare or C. difficile infection). ${ }^{671}$ A recent USA study using a multiplex PCR for gastrointestinal pathogens reported that $18.1 \%$ and $16.1 \%$ of samples from Crohn's disease and UC patients respectively were positive. Crohn's disease patients were more likely to have norovirus and Campylobacter, while UC patients were more likely to have bacterial infections, particularly Campylobacter, Plesiomonas and Escherichia coli (compared with non-IBD samples). ${ }^{672}$ The sensitivity of the molecular test used, the breadth of pick-up of the panel covered and the likelihood of false-positive infections because of asymptomatic carriage all warrant consideration when assessing the literature in this area. Nevertheless, a comprehensive infection screen considering clues from the history, and local infectious epidemiology, is an essential component of evaluating IBD disease flares.

\subsubsection{Clostridium difficile infection associated with IBD}

Clostridium difficile infection is more common in IBD than non-IBD populations, and in contrast to non-IBD populations, is less associated with PPI and antibiotic therapy. ${ }^{673674} \mathrm{C}$. difficile can also be a cause of chronic pouchitis. ${ }^{675}$

A Canadian population-based case-controlled study of 278 UC patients hospitalised due to a flare of colitis and tested for $C$. difficile infection identified $6.1 \%$ were positive. These patients had an increased risk of colectomy (adjusted OR 3.39) and preoperative $C$. difficile infection was associated with a higher risk of postoperative infectious complications (OR 4.76). ${ }^{218}$ Observational studies have shown approximately $20 \%$ of IBD patients with $C$. difficile infection require colectomy. ${ }^{214676}$ Outcomes in hospitalised IBD patients with $C$. difficile infection are worse with higher colectomy rates and higher mortality. ${ }^{215-218677678} \mathrm{~A}$

Statement 74 . We recommend that all patients presenting with acute flares of colitis should have stool cultures for enteroinvasive bacterial infections and stool Clostridium difficile assay (GRADE: strong recommendation, low-quality evidence). Microscopy and culture for amoebic or Shigella dysentery should be performed in patients with relevant travel history (Agreement: 93.5\%). 
Statement 75. We suggest that Clostridium difficile infection in acute severe ulcerative colitis is associated with significantly increased risk of colectomy and should be treated with oral vancomycin (GRADE: weak recommendation, low-quality evidence. Agreement: 93.2\%).
Statement 76. We suggest that patients with moderate to severe colitis, particularly those with corticosteroidrefractory disease, should have colonic biopsies to look for cytomegalovirus disease by H\&E staining, and preferably also immunohistochemistry or quantitative tissue PCR (GRADE: weak recommendation, low-quality evidence. Agreement: 93.3\%).

systematic review and meta-analysis showed that the 3-month colectomy risk was not increased by $C$. difficile infection (OR 1.35 ; $95 \%$ CI 0.68 to 2.67 ) either in IBD or non-IBD populations, but the colectomy risk after 1 year or more was higher for IBD overall (OR 2.23; 95\% CI 1.18 to 4.21 ) and UC specifically (OR 2.96; 95\% CI 1.19 to 7.34$){ }^{679}$

In non-IBD populations vancomycin is equally as effective as metronidazole for mild $C$. difficile, but has superior efficacy in severe disease. ${ }^{680}$ As such, Public Health England recommend vancomycin therapy in severe infection, as do others. ${ }^{219} 681$ It is not necessary to withhold corticosteroid treatment for acute severe colitis in this situation, and a decision regarding continuation of immunomodulator therapy should be taken on an individual basis (including the surgical team in the discussion). ${ }^{196} 681$ It is prudent not to escalate therapy or introduce rescue therapy with infliximab or calcineurin inhibitors in ASUC associated with $C$. difficile infection, as there is little or no data on safety in this situation.

\subsubsection{Cytomegalovirus infection in IBD}

Cytomegalovirus (CMV) in IBD can be diagnosed by the presence of typical CMV inclusions on H\&E stain, immunohistochemistry and/or tissue PCR. A recent systematic review suggested that blood-based testing and histology lack sensitivity to predict reactivation of CMV in the colon and so immunohistochemistry or tissue PCR remain essential in the detection of CMV IBD. ${ }^{682}$ In a retrospective case control study from the Mayo Clinic, the risk of CMV disease was independently associated with refractory disease, treatment with immunomodulators and age over 30 years. ${ }^{63}$ Other studies show an association of CMV with corticosteroid use, corticosteroid-refractory UC and leukopaenia. ${ }^{684-687} \mathrm{CMV}$ viraemia and blood PCR have poor sensitivity for diagnosis of CMV colitis, though specificity is high. ${ }^{688}$ Immunohistochemistry or quantitative PCR can be used to detect CMV on mucosal tissue, and may allow detection of CMV in patients who do not have inclusion bodies on H\&E staining. ${ }^{689-692}$ A recent systematic review has identified wide heterogeneity of the definitions of CMV infection and CMV intestinal disease. ${ }^{693}$ Due to this heterogeneity, the prevalence of CMV intestinal disease in IBD varies from $2 \%$ to $38 \%$. Tissue PCR has a sensitivity of $65 \%$ to $100 \%$ and a specificity of $40 \%$ to $100 \%$ whilst immunohistochemistry had a sensitivity of $93 \%$ and a specificity of $92 \%$ to $100 \%$. These are the preferred methods of diagnosis.

\subsubsection{Treatment of CMV in IBD}

Reactivation of CMV infection detected by serology is common in IBD patients receiving immunosuppression. ${ }^{694}$ Low-level reactivation may disappear without antiviral therapy. Small observational studies show benefit for treating colonic CMV disease in UC patients, particularly those who are steroid-refractory. ${ }^{695} 696$ Hospitalised patients with refractory UC, associated with CMV disease, treated with ganciclovir and requiring treatment with infliximab or ciclosporin did not have a worse outcome compared with those receiving ganciclovir only. ${ }^{697}$ Antiviral therapy in patients with a high density of CMV inclusions (biopsy specimens with five or more inclusions) reduces the need for surgery in the following year. ${ }^{698}$ Despite the lack of controlled trial data, a recent review concluded that there was sufficient evidence to support antiviral therapy in patients with moderate to severe colitis, particularly those resistant to steroids and colonic CMV reactivation by $\mathrm{H} \& \mathrm{E}$ staining with immunohistochemistry and/ or CMV tissue PCR. ${ }^{699}$ The treatment recommended by ECCO is intravenous ganciclovir $(5 \mathrm{mg} / \mathrm{kg}$ twice daily) for $3-5$ days, then oral valganciclovir (900 mg twice daily) for 2-3 weeks, but virology/microbiology advice should be taken regarding route and duration of therapy. ${ }^{700}$ Systemic CMV reactivation causing meningoencephalitis, pneumonitis, oesophagitis or hepatitis carry a poor prognosis and require prompt antiviral therapy and cessation of all immunosuppressive therapy. ${ }^{700}$

\subsection{Immunosuppressive therapy}

It is important that all patients receive written information prior to starting treatment, in addition to counselling regarding the risks of treatment and how these are minimised by monitoring. Patients have differing perceptions regarding treatment and this can significantly influence drug adherence. ${ }^{701}$ No study has addressed the impact of specific patient information, but a recent survey of IBD patients showed that patients access a wide variety of information sources about IBD and its treatment. ${ }^{702}$ Of these, information from the IBD team and written patient information material were the most widely used, and the most trusted by patients. Information can be provided by IBD teams in written form or by sign posting to official patient support websites that provide high-quality drug information for patients.

\subsubsection{Prevention of infection related to IBD and immunosuppressive therapy}

If evidence of active HBV, latent or active TB, HCV or HIV infection, seek specialist input prior to commencing immune modifying therapy.

All IBD patients should be screened at diagnosis for infection risk, as there is both an increased risk of infection as a result of the disease and a high likelihood of requiring immunosuppressive drug therapy (box 4). Patients without a clear history of

Statement 77. We suggest that CMV reactivation in the of ulcerative colitis or Crohn's colitis may be treated by intravenous ganciclovir $5 \mathrm{mg} / \mathrm{kg}$ twice daily while continuing conventional therapy with corticosteroids or rescue medication with infliximab or ciclosporin (GRADE: strong recommendation, very low-quality evidence). In rare cases with systemic disease (meningo-encephalitis, pneumonitis, hepatitis or oesophagitis), all immunosuppressive therapy should be stopped while CMV is treated (GRADE: weak recommendation, very low-quality evidence). (Agreement: $88.4 \%$ ). colonic mucosa of patients hospitalised with an exacerbation 
Good Practice Recommendation 14. All IBD patients to be initiated on immunosuppressive therapy should receive written information regarding the benefits, risks, side effects and monitoring of treatment (Agreement: 97.9\%).

chickenpox, shingles or receipt of two doses of varicella vaccine should be tested for varicella zoster virus (VZV) IgG. All patients with IBD should be screened for hepatitis B and C, and HIV. The prevalence of hepatitis $B$ and $C$ viral infection in IBD was similar to the general population in 315 patients from France (hepatitis B virus $2.54 \%$, hepatitis C virus $0.95 \%) .{ }^{703}$ A Dutch study showed that rates of screening for hepatitis $B$ remain suboptimal at $36-49 \% .^{704}$ Opportunistic testing for HIV should be done at the same time, as the condition is treatable and has public health implications. A panel of viral screening at diagnosis makes it more likely that patients can receive appropriate vaccinations with less delay to receiving immunomodulator therapy, which is now used in at least half of patients at some stage in their disease course. For the same reason, checking thiopurine methyltransferase (TPMT) status early reduces delays in starting thiopurines.

\subsubsection{Epstein Barr Virus}

There is increasing interest in Epstein Barr virus (EBV) infection in IBD patients because of the risk of severe complications, including haemophagocytic syndrome and post-mononucleosis type lymphomas after primary infection and haemophagocytic syndrome and post-transplant type lymphoma during latent infection. A Spanish tertiary care centre studied 1483 patients aged over 17 years between 2006 and 2016. ${ }^{705}$ They assessed EBV seroprevalence and seroconversion and documented those who developed complications while on azathioprine. EBV had a role in tumour development in three out of five patients on azathioprine, but only one (in a 66-year-old man) occurred during a primary infection. The authors conclude that the risk of haemophagocytic syndrome and lymphoma on thiopurine therapy is not restricted to young males. This is confirmed in a USA paediatric IBD follow-up cohort, where $3 / 5$ patients with haemophagocytic syndrome (all on thiopurines) were female. There was no association with use of infliximab or methotrexate (although numbers on methotrexate were much smaller). ${ }^{706}$ It remains unclear whether screening for EBV status should be done routinely in adults. ${ }^{707}$ In paediatric patients who are particularly at risk from primary EBV infection, there may be more justification for screening in order to avoid thiopurine use in those who are seronegative. Routine EBV testing prior to thiopurine therapy remains controversial.

\subsubsection{Infection risk in patients on anti-TNF therapy}

Meta-analysis of clinical trial data of 4135 patients receiving anti-TNF therapy as part of randomised clinical trials found a $0.9 \%$ incidence of opportunistic infection. ${ }^{708}$ This represented a two-fold increased risk of infections including TB, herpes

Statement 78. We suggest that blood tests for thiopurine methyltransferase status and screen for HBV, HCV and HIV (and VZV if no history of chicken pox, shingles or varicella vaccination) may be arranged once diagnosis is confirmed for all Crohn's disease and moderate to severe ulcerative colitis patients (GRADE: weak recommendation, very low-quality evidence. Agreement: 88.9\%).
Statement 79. We recommend that IBD patients commencing immunomodulators or biologics treatment should undergo screening for HBV, HCV and HIV (and VZV if no history of chicken pox, shingles or varicella vaccination), unless screened already at time of diagnosis (GRADE: strong recommendation, very low-quality evidence. Agreement: 88.9\%).

simplex, oral or oesophageal candidiasis, herpes zoster, CMV, EBV and Nocardia in IBD patients (RR 2.05; 95\% CI 1.10 to 3.85). The relative risk for TB was 2.52 (95\% CI 0.62 to 10.21 ). Pooled analysis of 2266 patients receiving adalimumab as part of clinical trials found that higher disease activity was associated with an increased risk of opportunistic infection, with a 31\% (HR 1.31; 95\% CI 1.04 to 1.64) increase accompanying every 100 point rise in CDAI. ${ }^{709}$ IBD patients over 50 years of age receiving immunosuppression are at highest risk of opportunistic infection. ${ }^{710} 711$

For patients starting biologics or immunosuppressive drugs, the viral screen (as recommended at diagnosis, see box 4) should be performed if not done initially, or if new risk factors have arisen since that time.

\subsection{Tuberculosis}

Screening for active or latent TB is essential prior to starting anti-TNF or other biologic therapy. A Dutch study suggested that screening rates for TB in IBD patients are high at 90-97\%, with $3 \%$ latent prevalence. ${ }^{704}$ Tuberculin skin test is likely to have false-negative results due to immunosuppressive therapy, so interferon gamma release assays (IGRA) should be used. A high rate of anergy to skin-based antigen tests was shown by immunising 82 consecutive IBD patients against tuberculin (TST) or control antigens. ${ }^{712}$ In this study no TST result was positive, but $71 \%$ of patients failed to respond to any antigen. Importantly, $83 \%$ of patients on steroid or immunomodulator therapy were anergic versus $43 \%$ not on these therapies $(p<0.002)$. A retrospective study of TB screening in 340 patients in the USA with 512 Quantiferon TB Gold (QFT-G) IGRA tests reported 1.5\% positivity, $2.7 \%$ indeterminate and $95.8 \%$ negativity. ${ }^{713}$ Only one case of tuberculosis reactivation occurred in 17 months of follow-up $(0.3 \%)$. This patient had an indeterminate test and was on immunosuppressive therapy prior to testing. No significant difference was seen in positivity rates between those on

Box 4 Infection and pretreatment checklist at diagnosis and prior to immunomodulator or biological therapy

- History of specific infections: HSV (oral, genital), VZV (chicken pox, shingles), tuberculosis.

- Immunisation status: BCG, diphtheria, tetanus, pertussis, Haemophilus influenzae type $\mathrm{B}$, polio, meningococcus, measles, mumps, rubella, pneumococcus, HPV, rotavirus, influenza, VZV/shingles. ${ }^{1285}$

- Prior exposure to TB: household contacts, prolonged stay or origin from endemic area.

- TB screening: patients considered for anti-TNF therapy should be screened for tuberculosis using a combination of clinical risk stratification, chest x-ray and interferon-gamma release assays.

- TPMT: check in all patients considered for thiopurine therapy.

- Serology: HBV, HCV, HIV and VZV (in patients without clear history of prior infection or prior vaccine). 
Statement 80 . We recommend that prior to commencing anti-TNF therapy, IBD patients should be screened for tuberculosis (TB) using a combination of clinical risk stratification, chest $\mathrm{x}$-ray and interferon-gamma release assays (IGRAs) (GRADE: strong recommendation, low-quality evidence. Agreement: 97.8\%).

immunosuppressive therapy and those not. Only moderate concordance was seen between TST and QFT-G in this study (kappa $=0.4152, p=0.0041)$. IGRA tests were evaluated in 125 adult patients with IBD in London, 90 of whom were anti-TNF naïve, with 35 on established anti-TNF therapy. ${ }^{714} 109$ $(87 \%)$ were BCG vaccinated. 98\% IGRA tests were negative, $1 \%$ indeterminate and $2 \%$ positive. This method of screening was deemed cost-effective in comparison to the 2005 British Thoracic Society guideline. ${ }^{715}$ No subsequent cases of TB were reported with a median follow-up of 24 months. A Korean study examined newly developed TB cases in IBD patients on established anti-TNF therapy. ${ }^{716}$ They described 25 cases in total (84\% pulmonary, $16 \%$ extrapulmonary), of whom $76 \%$ developed TB within 5 years of commencing anti-TNF despite previous negative screening for latent infection, and 12\% developed reactivation of latent infection after 3 months of chemoprophylaxis. Primary infection with TB on anti-TNF agents is therefore a significant risk, particularly in higher incidence areas, irrespective of baseline screening.

It has been proposed that extensive screening for $\mathrm{TB}$ using chest $\mathrm{x}$-ray and IGRA compared with chest $\mathrm{x}$-ray and TST is only cost-effective if latent prevalence is $>12 \%$ or false positivity rate of TST is $>20 \% .{ }^{717}$ In low prevalence areas, patients should have a risk assessment questionnaire and chest $x$-ray, with IGRA test if at increased risk.

5.2.1.2.2 Strongyloides stercoralis

Strongyloides stercoralis is an intestinal parasitic infection affecting tens of millions of people globally. It has been estimated the infection may be present in up to $10-40 \%$ of the population in tropical and subtropical countries, rising to $60 \%$ in the poorest socioeconomic communities where environmental conditions favour spread of infection. ${ }^{718}$ Patients with HIV/AIDS have twice the risk of infection compared with non-HIV populations (OR 2.17; 95\% CI 1.18 to 4.01). Immunocompromised patients, particularly those on systemic steroids, are at risk of potentially fatal hyperinfection syndrome, characterised by increased parasite burden leading to GI bleeding, pneumonia, sepsis or meningitis. ${ }^{719}$ Blood eosinophilia is a common finding in patients with S. stercoralis infection though is not present in all patients. ${ }^{720} \mathrm{~S}$. stercoralis serology is unreliable in immunocompromised individuals. Treatment is with ivermectin or albendazole.

Risks and management of other opportunistic infections are well-covered in the ECCO guidance. ${ }^{700}$

\subsubsection{Vaccination}

A vaccination history should be taken both at diagnosis and prior to starting immunosuppressive therapy (see box 4). General advice regarding vaccination is given in box 5 .

5.2.1.3.1 Non-live vaccines

IBD patients have a greater risk of contracting influenza than non-IBD populations (incidence rate ratio $1.58 ; 95 \%$ CI 1.49 to $1.68)$ and are more likely to require admission to hospital. ${ }^{721}$ Annual influenza vaccination is recommended for all immunosuppressed patients, ${ }^{1700} 722$ although vaccine efficacy may be reduced, particularly in those on anti-TNF therapy. ${ }^{723}$ It should
Statement 81 . We suggest that IBD patients who have travelled for long periods or lived in endemic areas may be at increased risk of parasitic infections, and may have Strongyloides serology, and eosinophil count checked prior to commencing anti-TNF therapy (GRADE: weak recommendation, very low-quality evidence. Agreement: 90.9\%).

be remembered that the nasal influenza vaccine contains live virus and so should be avoided, with the injection favoured instead.

Assessment of hepatitis B serology and vaccination in all seronegative patients at diagnosis is recommended in ECCO guidelines, although the value of this in low prevalence countries has been questioned, and in the UK it may be more appropriate to offer this to high-risk groups on the basis of lifestyle, occupation or other factors. ${ }^{724}$ Efficacy of vaccination may be impaired in active $\mathrm{IBD}^{725}$ and in those on immunosuppressive drugs. ${ }^{726} 727$ After hepatitis $\mathrm{B}$ vaccination, anti-HBs response should be measured as higher doses may be required. Accelerated double-dose vaccination in IBD has been shown to improve response, with doubledose Engerix-B vaccine at 0,1 and 2 months. ${ }^{728}$

Pneumococcal vaccination is also affected by immunosuppression and should ideally be administered at least 2 weeks before starting immunomodulators. Three pneumococcal vaccines are licensed in the UK: pneumococcal polysaccharide vaccine (PPV23, containing polysaccharide from 23 capsular types of pneumococcus) and two variants of pneumococcal conjugate vaccine (PCV13 and PCV10, containing polysaccharide from 13 and 10 capsular types of pneumococcus). ${ }^{729}$ The current recommendation for adults on immunosuppression is a single dose of PCV13 followed by PPV23 at least 2 months later; however, we recommend reviewing the Green Book for further detail. Booster pneumococcal vaccination with PPV23 is recommended after 5 years in patients who are asplenic, who have reduced splenic function or chronic renal disease. It seems reasonable to give boosters to patients on long-term immunomodulator therapy also, although there is little evidence in this group.

5.2.1.3.2 Live vaccines

Following live vaccination, the UK Department of Health currently recommends allowing 4 weeks for the immune response to be established prior to commencing immunosuppressive or biologics therapy. ${ }^{730}$ Live vaccination should be avoided during biologics therapy and for a minimum of 3 months after stopping. ${ }^{731-733}$ The evidence base for the 3-month period is poor, and while drug blood levels will be minimal by this time, it is unclear whether alterations to white cell populations may have more persistent subtle effects on immunity. The USA Centre for Disease Prevention and Control states in their IDSA guidelines that zoster live vaccination can be considered for patients aged 60 or over while on treatment with low-level immunosuppression

Statement 82 . We recommend that a vaccination history should be obtained and vaccinations updated for all patients with Crohn's disease, those with moderate to severe ulcerative colitis at diagnosis, and prior to commencing immunomodulator or biologics in all patients. Live vaccinations may be given at least 4 weeks before starting and at a minimum of 3 months after stopping, but not while receiving immunosuppressive therapy (GRADE: strong recommendation, very low-quality evidence. Agreement: 93\%). 
Statement 83 . We recommend that IBD patients receiving immunomodulators or biologics should receive influenza vaccination each autumn, and pneumococcal vaccination with a booster after 5 years (GRADE: strong recommendation, very low-quality evidence. Agreement: 95.5\%).

(defined as prednisolone doses $<20 \mathrm{mg}$, methotrexate weekly dose $\leq 0.4 \mathrm{mg} / \mathrm{kg}$, azathioprine $\leq 3 \mathrm{mg} / \mathrm{kg} /$ day or mercaptopurine $\leq 1.5 \mathrm{mg} / \mathrm{kg} /$ day). ${ }^{734}$ In the UK the age recommendation for zoster vaccine is at age 70 or up to the age of 79 if not given at age 70 , and the Green Book also advises that shingles vaccination may be administered to those on low-level immunosuppression (defined as $\leq 20 \mathrm{mg}$ prednisolone/day for $>14$ days, either alone or in combination with low-dose non-biological oral immunomodulators (methotrexate $\leq 25 \mathrm{mg} /$ week, azathioprine $\leq 3 \mathrm{mg}$ / $\mathrm{kg} /$ day or mercaptopurine $\leq 1.5 \mathrm{mg} / \mathrm{kg} /$ day)). ${ }^{735}$

The UK Department of Health Green Book vaccination guide Chapter $6^{730}$ also suggests that all live vaccines can be considered for those on low-dose immunosuppression as defined above, including low-dose immunosuppressive drugs in combination with prednisolone up to $20 \mathrm{mg}$. There is evidence from a small controlled study that the use of zoster vaccine in IBD patients on low-level immunosuppression does not result in adverse effects, although antibody titres were somewhat lower than those in vaccinated IBD patients not taking immunosuppressive drugs. ${ }^{736}$ Live vaccination must not be given to those taking biologics, and should only be undertaken for those on low-level immunosuppression after careful consideration of the risks and benefits in conjunction with the patient.

Box 5 General considerations regarding vaccination

- Live vaccines are contraindicated if your patient is on immunosuppression or with significant protein calorie malnutrition: live vaccines include BCG, attenuated (oral) influenza vaccine, measles, mumps and rubella (MMR), polio, rotavirus, oral typhoid Ty21a, varicella zoster, yellow fever. Immunosuppressive therapies include: glucocorticoids (prednisolone $\geq 20 \mathrm{mg} /$ day or equivalent for 2 weeks or more), thiopurines, methotrexate, biological therapy and tofacitinib.

- Shingles vaccination (current UK age recommendation is at age 70 years or up to the age of 79 years if not given at age 70 years) may be administered to those on low-level immunosuppression (defined as $\leq 20 \mathrm{mg}$ prednisolone/ day for $>14$ days, either alone or in combination with lowdose non-biological oral immunomodulators (methotrexate $\leq 25 \mathrm{mg} /$ week, azathioprine $\leq 3 \mathrm{mg} / \mathrm{kg} /$ day or mercaptopurine $\leq 1.5 \mathrm{mg} / \mathrm{kg} /$ day). ${ }^{735}$

- Immunomodulators should be withheld for 4 weeks after live vaccine administration.

- Live vaccines should be avoided for at least 3 months after discontinuing treatment with immunosuppressive therapies above.

- Infants exposed to biologics in utero should not receive live vaccines for 6 months after birth.

- IBD patients on immunosuppressant therapy should receive pneumococcal vaccine and annual influenza vaccination (prior to starting treatment if possible) with a single pneumococcal booster at 5 years.
The Green Book advises that live vaccination should be delayed until 6 months of age in children exposed in utero to biologics. $^{730}$

\subsubsection{Drug management: thiopurines}

A checklist of considerations when commencing thiopurines is shown in box 6 , and table 10 gives a guide on interpretation of thiopurine metabolites during dose optimisation. Practical management of thiopurine side effects is described in box 7 .

\subsubsection{Thiopurine methyltransferase and NUDT15}

20-30\% of IBD patients stop thiopurine treatment because of side effects. While overall, thiopurine methyltransferase (TPMT) activity does not predict adverse effects, the subset of patients with low TPMT activity are at increased risk of discontinuing thiopurines due to adverse effects. In a prospective study of TPMT-directed versus standard therapy, patients with TPMT variants fared significantly better if dosed according to TPMT status $(2.6 \%$ haematological adverse effects in TPMT-directed therapy versus $22.9 \%$ in undirected group, RR $0.11,95 \% \mathrm{CI}$ 0.01 to 0.85$).^{737}$ In a prospective evaluation of TPMT activity in 207 patients with IBD commenced on azathioprine, patients with heterozygous TPMT deficiency were much more likely to withdraw due to adverse effects compared with those with wildtype TPMT status $(79 \%$ vs $35 \%, \mathrm{p}<0.001) .{ }^{738}$ There is evidence that TPMT measurement in all patients starting thiopurine therapy is cost-effective. ${ }^{739} 740$

The usual dose of azathioprine is $2-2.5 \mathrm{mg} / \mathrm{kg}$ daily and for mercaptopurine $1-1.25 \mathrm{mg} / \mathrm{kg}$ daily for patients with normal TPMT activity (box 6). There is a very high risk of thiopurine-induced myelosuppression in patients with absent TPMT activity (homozygous or compound heterozygous TPMT deficiency). While very low dose (5\% of usual target) has been proposed, a thiopurine should generally be avoided in this group. In those with heterozygous TPMT deficiency, however, 50\% of standard thiopurine dose is associated with improved tolerance. ${ }^{737}$

Genetic variation in NUDT15 has now also been described in association with myelosuppression. This was originally described in East Asians ${ }^{741}$ but has now also been described in IBD patients of European ancestry. ${ }^{742}$ Recent CPIC guidelines recommend NUDT15 testing, particularly for Asian patients, with consideration of dose reduction or thiopurine avoidance. ${ }^{742}$

\subsubsection{Starting dose for thiopurines}

Azathioprine and mercaptopurine should be started at the full dose. There is no evidence that starting at low doses and then gradually increasing up to target improves safety or tolerance, and low-dose initiation may cause significant delay in achieving the correct target dose. ${ }^{743}$

\subsubsection{Renal excretion of thiopurines}

Thiopurine metabolites are renally excreted. Thiopurines should be used with caution in renal impairment with $75 \%$ of usual dose given if creatinine clearance is $10-50 \mathrm{~mL} / \mathrm{min}$, and $50 \%$ of usual dose if $<10 \mathrm{~mL} / \mathrm{min}^{744}$

\subsubsection{Cervical neoplasia and thiopurines}

A number of large studies have found an increased risk of cervical dysplasia among women with IBD compared with controls. ${ }^{745}$ This risk is mainly confined to those on oral immunosuppressive therapy, including corticosteroids, and those who smoke. ${ }^{746-749}$ A meta-analysis gives an overall modest increase in risk, with 


\section{Box 6 Initiation of thiopurines}

\section{Before starting:}

- All patients should receive verbal and written information about their medication

- Baseline FBC, U\&E and LFT measurement

- If available test NUDT15 genotype

- Screen for HCV, HBV, HIV, refer if positive, consider HBV vaccination if naïve

- Check VZV immunity and vaccinate if low

- Vaccinate for influenza and pneumococcal vaccine

- Check cervical screening up to date

- Check TPMT and start at target dose once result available. Normal TPMT: $2 \mathrm{mg} / \mathrm{kg}$ azathioprine or $1 \mathrm{mg} / \mathrm{kg}$ mercaptopurine. Low: $1 \mathrm{mg} / \mathrm{kg}$ azathioprine or $0.5 \mathrm{mg} / \mathrm{kg}$ mercaptopurine. Very low: avoid thiopurine

\section{Monitoring:}

- FBC, U\&E and LFT at least at weeks $2,4,8$, and 12 , and then at least 3-monthly

an OR of 1.34 (95\% CI 1.23 to 1.46$)$ for IBD patients on immunosuppression. ${ }^{750}$ As a consequence, all women should be encouraged to participate in national cervical cancer screening programmes; in the UK this is currently 3-yearly from age 25-49 and 5-yearly from age 50-64 years.

\subsubsection{Drug monitoring for thiopurines}

Measurement of the thiopurine metabolites (thioguanine nucleotides (TGN) and methylmercaptopurine (MeMP)) offers a benefit over standard haematological and biochemical monitoring by detecting non-adherence to therapy, inadequate dosing or an unnecessarily high dose of thiopurine, ${ }^{751752}$ as shown in table 10 . Furthermore, detection of a skewed metabolism towards excessive thiopurine methylation determines a group of patients at risk of poor response and hepatotoxicity.

Although one study has suggested cost-effectiveness of metabolite monitoring in improving sustained response to thiopurines, ${ }^{753}$ it is unclear whether routine measurement in all patients on thiopurines is beneficial, owing to wide variation in levels (as much as fivefold intra-patient variation in one study $\left.{ }^{754}\right)$. Although TGN levels of $230-400 \mathrm{pmol} / 8 \times 10^{8}$ erythrocytes have been associated with better response and MeMP levels $>5000 \mathrm{pmol} / 8 \times 10^{8}$ erythrocytes with more liver toxicity ${ }^{755}$ appropriate thresholds are unclear and small prospective studies have not shown clinical benefit. ${ }^{756-758}$ In patients on combination therapy with infliximab and thiopurines, a lower target level of TGN of $125 \mathrm{pmol} / 8 \times 10^{8} \mathrm{RBCs}$ may be adequate to achieve therapeutic levels of infliximab, ${ }^{759}$ with a further study suggesting a target level of $>105 \mathrm{pmol} / 8 \times 10^{8} \mathrm{RBCs}^{760}$

\subsubsection{Low-dose thiopurines with allopurinol}

Individuals whose methylation pathways predominate (hypermethylators) create less TGN and more methylated metabolites (MeMP). They therefore have lower thiopurine efficacy and a higher risk of side effects, particularly hepatotoxicity. ${ }^{761}$ This pattern of metabolism can be picked up as early as 4 weeks after starting the drug (ie, long before therapeutic effect is anticipated) and circumvented by switching to a low-dose thiopurine (25-33\% of usual dose) with allopurinol $100 \mathrm{mg}$ co-prescription regimen, avoiding toxicity and increasing efficacy. Allopurinol achieves this optimisation of thiopurines by reducing methylation and increasing levels of the target metabolite, TGN. ${ }^{762763}$ The accepted ratio of MeMP to TGN at which a switch is indicated is MeMP:TGN $\geq 11 .^{764}$ One randomised study comparing low-dose thiopurine with allopurinol with thiopurine monotherapy showed a higher proportion of patients were able to avoid steroid or biologic on combination treatment: $69.6 \%$ vs $34.7 \%$, RR 2.1 (95\% CI 1.07 to 4.11$){ }^{765}$ Withdrawal rates due to adverse events on combination therapy were $30.4 \%$ versus $47.8 \%$ in those receiving monotherapy (RR $1.47,95 \% \mathrm{CI} 0.76$ to 2.85 ). Other uncontrolled series show consistently that adverse effects experienced on monotherapy can frequently be circumvented by low-dose thiopurine with allopurinol, ${ }^{766-769}$ and clinical responses improved. ${ }^{766} 770-772$

\subsubsection{Thiopurine toxicity}

TPMT testing will only predict a proportion of early haematological toxicity on thiopurines, ${ }^{737773}$ and no current pretreatment test will rule out future biochemical toxicity on a thiopurine. Hence, early intensive monitoring for haematological and biochemical toxicity is recommended in all patients with blood tests for full blood count, renal and liver biochemistry at 2, 4, 8

\begin{tabular}{|c|c|c|c|}
\hline TGN (pmol/ $\left.8 \times 10^{8} \mathrm{RBCs}\right)$ & 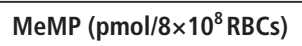 & Interpretation & Treatment change to consider \\
\hline Undetectable & Undetectable & Poor/variable compliance & $\begin{array}{l}\text { Patient education } \\
\text { Rarely poor absorption }\end{array}$ \\
\hline Low $(<235)$ & Low/normal $(<5700)$ & Subtherapeutic dosing & Uptitrate dose then repeat metabolites \\
\hline Low $(<235)$ & $\begin{array}{l}\text { High }(>5700 \text { or } \\
\text { MeMP: TGN }>11)\end{array}$ & $\begin{array}{l}\text { Thiopurine hypermethylator } \\
\text { (occurs in up to } 20 \% \text { of patients with normal } \\
\text { TPMT) }\end{array}$ & $\begin{array}{l}\text { Reduce dose to } 25-33 \%+\text { start allopurinol } 100 \mathrm{mg} / \text { day, then } \\
\text { repeat metabolites }\end{array}$ \\
\hline Therapeutic (235-450) & Normal $(<5700)$ & $\begin{array}{l}\text { Therapeutic (if responding) } \\
\text { Thiopurine resistant (if not responding) }\end{array}$ & $\begin{array}{l}\text { If responding, continue current dose } \\
\text { If not responding, change drug category }\end{array}$ \\
\hline Therapeutic (235-450) & High $(>5700)$ & Possible supratherapeutic dosing & $\begin{array}{l}\text { Attempt dose reduction and repeat in } 4 \text { weeks. If non-responding } \\
\text { and TGN low end of normal range, consider low dose azathioprine } \\
\text { with allopurinol as above }\end{array}$ \\
\hline High $(>450)$ & High $(>5700)$ & Supratherapeutic dosing & Reduce dose then repeat metabolites \\
\hline
\end{tabular}

Adapted from Goel et al. ${ }^{1286}$

MeMP, methylmercaptopurine nucleotides; RBC, red blood cells; TGN, 6-thioguanine nucleotides; TPMT, thiopurine methyltransferase. 


\section{Box 7 Managing side effects of thiopurines}

\section{Pancreatitis:}

- Do not give AZA or MP again, even at low dose. High chance of recurrence

Nausea and vomiting:

- Try switching from AZA to MP; or continue drug with split dosing

- If recurs then consider low dose (25-33\% of standard dose) AZA or MP+allopurinol $100 \mathrm{mg}$

Flu-like symptoms:

- Unlikely to resolve on switching from AZA to MP; some evidence for AZA/MP+allopurinol $100 \mathrm{mg}$

- If convincing early hypersensitivity reaction, there is high risk of recurrence and so consider switch to alternative class of drug

\section{Newly abnormal LFTs:}

- Stop and check thiopurine metabolites

- Withhold until LFTs abnormality resolves

- If not resolving, investigate as usual

- Once resolved, re-challenge with low dose AZA/ $\mathrm{MP}+$ allopurinol $100 \mathrm{mg}$. This is particularly likely to work if the original metabolites showed hypermethylation (High MeMP levels)

\section{Myelotoxicity:}

- Check thiopurine metabolites and alter dosage according to table 10

- Monitor white cell counts closely

- If total white cells $<3.5 \times 10^{9} / \mathrm{L}$ or neutrophils $<2 \times 10^{9} / \mathrm{L}$, withhold thiopurine until counts correct above this level

- If neutrophils $<1 \times 10^{9} / \mathrm{L}$, patients should be warned to present for antibiotics \pm GCSF if febrile

- If TGN high, then restart at lower dose once abnormality has resolved and monitor haematology and thiopurine metabolites carefully

- If MeMP high then consider restarting low-dose thiopurine with allopurinol $100 \mathrm{mg}$

- If TGN low or normal then likely to reoccur. Advise to stop thiopurine in this circumstance

AZA, azathioprine; MP, mercaptopurine; AZA/MP, azathioprine or mercaptopurine; GCSF, granulocyte colony stimulating factor; MeMP, methylmercaptopurine nucleotides; TGN, 6-thioguanine nucleotides.

and 12 weeks of therapy, ${ }^{774}$ with ongoing 12 -weekly blood monitoring as toxicity can occur at any stage during therapy. ${ }^{774} 775$ Bloods should be repeated 2 weeks after all dose increases. In a Spanish nationwide database study of nearly 4000 patients, the cumulative incidence of thiopurine side effects was $26 \%$. Nausea was seen in $8 \%$, hepatotoxicity in $4 \%$, myelotoxicity in $4 \%$ and pancreatitis in $4 \%$ (pancreatitis occurring more often in Crohn's than UC, and more likely if also taking prednisolone). ${ }^{776}$ While a genetic predictor of thiopurine-induced pancreatitis has recently been discovered (the HLA-DQA1*02:01-HLA-DRB1*07:01

Statement 84. We recommend that all IBD patients considered for thiopurine therapy should have assessment of thiopurine methyltransferase (TPMT) status (GRADE: strong recommendation, moderate-quality evidence. Agreement: 100\%).
Statement 85 . We recommend that thiopurines should be avoided in patients with low TPMT activity. The dose of thiopurine should be reduced to $50 \%$ in those with intermediate thiopurine activity. Daily dosage should also be reduced in patients with significant renal impairment (GRADE: strong recommendation, moderate-quality evidence. Agreement: 100\%).

haplotype), routine screening is not considered cost-effective at present.

In patients with side effects to azathioprine, potential strategies include split dosing, a switch to mercaptopurine ${ }^{778}$ and use of low-dose thiopurine/allopurinol (as detailed in Section on Low-dose thiopurines with allopurinol 5.2.2.6). The success of such strategies varies between type of adverse reaction, and more details are provided in box 7.

\subsubsection{Drug use: methotrexate}

Gastrointestinal and liver toxicity of methotrexate is reduced by administration of folic acid, either $1 \mathrm{mg}$ daily or $5 \mathrm{mg}$ weekly, traditionally taken 1 or 2 days after the methotrexate dose. ${ }^{779} 780$ Methotrexate has comparable safety to thiopurines. ${ }^{78178}$ Risk of cirrhosis is much lower than previously thought and routine liver biopsy after prolonged use is not necessary. Abnormalities of liver function can be transitory; in one study of 87 IBD patients (with a cumulative methotrexate dose of $1813 \mathrm{mg}$ ) this occurred in $24 \%$ of patients treated, but many normalised without stopping methotrexate and only 5\% had to stop the drug. ${ }^{783}$ In this study 17 liver biopsies were performed, none showing advanced liver fibrosis or cirrhosis. Another study of 518 patients on methotrexate for inflammatory disease $(24 \%$ with Crohn's) assessed liver fibrosis by transient elastography. ${ }^{784}$ Six percent (31 patients) had FibroScan results suggesting severe liver fibrosis (4\% of the Crohn's disease patients). Only 13 went on to liver biopsy, with just under half having severe fibrosis confirmed histologically. On multivariable analysis, BMI $>28 \mathrm{~kg} / \mathrm{m}^{2}$ and alcohol intake $>14$ drinks per week were independent predictors of FibroScan values $>7.9 \mathrm{kPa}$. Patients with these additional risk factors should be screened using transient elastography. For all patients, methotrexate should be stopped if transaminases exceed twice the upper limit of normal. Pulmonary toxicity in the form of acute interstitial pneumonitis presents with dyspnoea, dry cough and fever, and is rare. Most cases are reversible on withdrawal of methotrexate. ${ }^{781}$ All patients commencing methotrexate should have a baseline chest $x$-ray.

\subsubsection{Methotrexate and pregnancy}

Methotrexate is teratogenic and should not be given to women of childbearing age without detailed discussion and agreement about the importance of assured contraception during therapy, and for 6 months after stopping the drug. If there are concerns about reliability of adherence to effective contraception, then alternative therapy should be used. Information on 63 pregnancy outcomes of women exposed to low-dose methotrexate in the first trimester reported that 30\% underwent termination

Statement 86 . We recommend that women with IBD commencing thiopurine therapy should be advised to participate in a national cervical screening programme (GRADE: strong recommendation, very low-quality evidence. Agreement: 95.7\%). 
Statement 87. Thiopurine metabolites (TGN and MeMP) can be used to optimise drug dosing. We suggest that metabolite monitoring may be used for those with inadequate response to therapy or toxicity, but should not be a substitute for routine monitoring blood tests (GRADE: weak recommendation, low-quality evidence. Agreement: 92.9\%).
Statement 89 . We recommend that IBD patients initiating thiopurine or methotrexate therapy should have baseline $\mathrm{FBC}$, U\&E and LFT measurement, with monitoring of these bloods at least at weeks 2, 4, 8 and 12, and then at least 3-monthly with monitoring for side effects (GRADE: strong recommendation, low-quality evidence. Agreement: 100\%). of pregnancy, and of the remaining women, $25 \%$ had miscarriage and $12 \%$ of those going to term had a baby with congenital abnormality (including one child with multiple skeletal abnormalities). ${ }^{785}$

There is no firm evidence to support the recommendation that men should discontinue methotrexate pre-conception. Two cohort studies of men receiving the drug for rheumatoid arthritis are reassuring regarding risk of preterm birth and fetal malformation. In a study of men with immunosuppressive and biological drug therapy for rheumatic diseases (of whom 100 were taking methotrexate), there was no evidence of an increase in adverse pregnancy outcomes. ${ }^{786}$ A further study of 113 pregnancies where the male partner had taken low-dose methotrexate for rheumatological disorders also showed no increase in risk. ${ }^{787}$ A Danish nationwide cohort study showed no adverse birth outcomes in children born to 193 men taking methotrexate. $^{788}$

\subsubsection{Drug management: anti-TNF including biosimilars \\ 5.2.4.1 Choice of anti-TNF agent}

Data regarding differences in efficacy between available anti-TNF drugs are not available from direct head-to-head trials. Existing trial data are difficult to compare indirectly owing to differences in study design and patient populations. Nevertheless, several systematic reviews and meta-analyses have reported minimal or inconsistent efficacy differences in Crohn's disease, with possible superiority of infliximab compared with other anti-TNF agents in the induction phase of treatment of UC. ${ }^{161789-791}$ No significant differences in safety profile have been reported, ${ }^{792}$ although the increased immunogenicity of infliximab potentially increases the need for co-prescription of an immunomodulator, with resulting effects on safety.

A retrospective study using USA Medicare data showed no difference between adalimumab and infliximab treatment for Crohn's disease in the proportion of patients continuing treatment at 6 months, nor in rates of surgery or hospitalisation. ${ }^{793}$ In a retrospective study of 3205 biologic-naïve patients with Crohn's disease from a USA national administrative claims database, those who received infliximab had a small but significantly lower risk of Crohn's disease-related hospitalisation, surgery or need for steroids compared with those started on adalimumab (adjusted HRs of 0.8 (95\% CI 0.66 to 0.98 ), 0.76 (95\% CI 0.58 to 0.99 ) and 0.85 (95\% CI 0.75 to 0.96 ), respectively). The study used propensity scores to match the cases as there were significant differences at baseline between the groups. $^{794}$

Statement 88. We suggest that low-dose thiopurines (25-33\% of usual dose) used in combination with allopurinol $100 \mathrm{mg}$ may be considered in patients with thiopurine hepatotoxicity, nausea or flu-like symptoms, or those who are hypermethylators (GRADE: weak recommendation, low-quality evidence.

Agreement: 81.4\%).
In a nationwide Danish IBD registry-based propensity score-matched cohort study, when used as a first anti-TNF in Crohn's disease, adalimumab-treated patients had a higher rate of all-cause hospitalisations (HR 1.84, 95\% CI 1.18 to 2.85), a trend towards higher UC-related hospitalisation and a higher rate of serious infection requiring hospitalisation (HR 5.11, 95\% CI 1.20 to 21.80 ) relative to infliximab. The risk of abdominal surgery was not different between the two treatment groups. ${ }^{795}$ In the prospective randomised SWITCH trial 47\% of Crohn's disease patients in remission on standard dose infliximab who were switched to adalimumab $40 \mathrm{mg}$ every other week either required dose escalation or switch back to infliximab to maintain remission. ${ }^{796}$ Combination infliximab plus azathioprine and adalimumab also seemed to be more effective than certolizumab in inducing remission in a recent network meta-analysis (OR 3.1 (95\% CI 1.4 to 7.7 ) and 2.1 (95\% CI 1.0 to 4.6 ) respectively), although both appeared equivalent in maintaining remission (and both were superior to thiopurine alone). ${ }^{456}$

In a Swiss study, patients offered a choice between treatments stated that ease of use was the most important consideration with a majority favouring adalimumab, but also cited time required for treatment, time interval between doses, evidence of efficacy and fear of injections as reasons for expressing preference. ${ }^{797} \mathrm{~A}$ Korean study cited the presence of a doctor as the reason why a majority favoured infliximab. ${ }^{798}$ Relative costs and infusion unit availability issues/access should also be considered (see box 2).

\subsubsection{Biosimilar anti-TNF drugs}

The introduction of biosimilar medicines has introduced competition into the market for biological medicines with significant cost reductions. Biosimilar medicines are approved for use in the EU by the European Medicines Agency on the basis of high similarity of structure, purity and biological activity to the originator biological medicine, with clinical evidence of comparable safety and efficacy for at least one therapeutic indication. ${ }^{799}$ Based on all the scientific data (comparability studies in quality, non-clinical and clinical areas), extrapolation to other indications (where the drug is believed to have a similar mechanism of action) can then be made for the biosimilar. The European Medicines Agency does not regulate interchangeability, switching or substitution, which is the remit of national bodies. It is recommended that biosimilars are prescribed by brand name to ensure full traceability and accountability. Patients should be fully informed

Statement 90. We recommend that, due to teratogenic and embryotoxic effects of methotrexate, prior to conception women should discontinue methotrexate for 6 months (GRADE: strong recommendation, low-quality evidence). If patients become pregnant on methotrexate then the drug should be discontinued and high dose folic acid (15 mg daily) provided for at least 6 weeks. We suggest that men taking methotrexate may not need to discontinue treatment prior to conception (GRADE: weak recommendation, low-quality evidence. Agreement: 92.7\%). 
Statement 91. We recommend that biosimilar infliximab may be used for IBD patients starting treatment (GRADE: strong recommendation, low-quality evidence). We recommend that patients already on originator infliximab can be switched to biosimilar infliximab if in stable response or remission (GRADE: strong recommendation, moderate-quality evidence. Agreement: $97.7 \%)$.

about the biological medicine they are using, particularly if there is a change to a biosimilar from the originator molecule. Automatic substitution is inappropriate, as all changes should be made with the full agreement and supervision of the prescribing physician. ${ }^{800}$ Extrapolation by indication is likely to be required for biosimilar use in IBD (comparative trials for regulatory purpose have not, to date, been performed in IBD) and should be evaluated by regulators on a case-by-case basis. Switching from originator biological medicine to biosimilar should also remain a clinical decision to be made by the physician and patient on an individual basis supported by the scientific evidence and by national recommendation. ${ }^{801}$ Currently, scientific evidence is lacking for reverse switching (back from biosimilar to originator), multiple switching and cross-switching. ${ }^{802}$ The effect on safety, efficacy and immunogenicity is not known and these practices should be avoided unless there is a specific need identified by the treating physician for an individual patient.

Biosimilar infliximab is already in use in IBD, and there is evidence available to support its safety and effectiveness. The NOR-SWITCH investigators studied 482 patients with Crohn's disease, UC, spondyloarthritis, rheumatoid arthritis, psoriatic arthritis or plaque psoriasis who were randomised to continue originator infliximab or switch to CT-P13 biosimilar infliximab. ${ }^{803}$ Switching to biosimilar infliximab was non-inferior over 52 weeks with $26 \%$ experiencing disease worsening with continuation of originator infliximab versus $30 \%$ of those switched to CT-P13. A potential limitation of this study was a selected $15 \%$ non-inferiority margin. NOR-SWITCH was not powered to examine non-inferiority in individual disease groups, though the CI was close to inferiority for CT-P13 in Crohn's disease. Excluding patients who had detectable anti-drug antibodies (ADAs) at baseline, the incidence of ADAs was 7\% for infliximab originator and 8\% for CT-P13, with no significant difference in immunogenicity. Two single-centre observational studies of switching from Remicade to CT-P13 in Crohn's disease and UC have also shown good short-term outcomes. ${ }^{804} 805$ Cohort studies of switching to biosimilar infliximab for psoriasis, ankylosing spondylitis and rheumatoid arthritis have all confirmed comparable efficacy and tolerability following switching. ${ }^{806-809}$

Biosimilar versions of adalimumab became available in the UK in late 2018. Comparative clinical effectiveness and safety trials against originator adalimumab have been conducted in rheumatoid arthritis and psoriasis. Principles as discussed above should be used in decision-making about the use of all biosimilar adalimumab drugs in IBD. Post-marketing surveillance and outcomes data collected in national registries are essential to look for safety evidence, including immunogenicity, with biosimilar medicines.

\subsubsection{Assessment after anti-TNF induction therapy}

Clinical response to anti-TNF agents is seen early in Crohn's disease and UC. Non-responders to induction therapy seem to have little benefit from sustained treatment, as shown in the ACCENT and CHARM studies. ${ }^{476} 486 \mathrm{~A}$ clinical assessment should be made 2-4 weeks after loading doses have been
Good Practice Recommendation 15. All IBD patients should be reviewed 2-4 weeks after completing loading doses of anti-TNF therapy to assess response and optimise maintenance dosing based on clinical response and measures such as serum drug and anti-drug antibody concentrations, blood inflammatory markers, faecal biomarkers or endoscopy (Agreement: 82.5\%).

given. For those who have responded, dose optimisation is recommended. If a response is unclear, then measurement of biomarkers, serum C-reactive protein and faecal calprotectin, or comparison of disease activity scores or PROMs with baseline values, may be helpful. For those with complete lack of response, treatment should be discontinued and alternatives considered (choosing a different class of therapy). Further data are needed on the use of drug level monitoring during induction to guide therapy. Much of the data on drug levels after induction is at a later time point, such as 14 weeks after starting infliximab in the TAILORIX study (see below) ${ }^{810}$ Observational data in UC show that higher infliximab drug levels at weeks 2 and 6 during induction therapy are associated with early mucosal healing, but there are no prospective studies using drug levels at these early time points to guide dose adjustment. ${ }^{811}$

\subsubsection{Drug levels of infliximab and adalimumab}

Trough concentrations during and following induction therapy have been shown to correlate with response to therapy for both infliximab and adalimumab. ${ }^{812}$ In an analysis of infliximab data from the ACT 1 and ACT 2 UC studies, serum levels at week 8 correlated with clinical response, remission and mucosal healing. ${ }^{250}$ This is confirmed in many studies, also showing that undetectable serum levels are often associated with anti-drug antibodies and worse clinical outcomes. ${ }^{813-815}$ The randomised TAILORIX trial assessed use of drug levels in 122 luminal Crohn's disease patients starting infliximab with an immunomodulator, and did not show benefit for treatment intensification starting at week 14 based on regular clinical assessment combined with drug levels and biomarker measurement when compared with clinical assessment only. ${ }^{810}$ The TAXIT study recruited IBD patients on stable maintenance infliximab therapy to evaluate trough infliximab levels to guide dosing. ${ }^{816}$ In the initial phase, all had their dose optimised with a target level of $3-7 \mu \mathrm{g} / \mathrm{mL}$. Patients with low trough levels had their dose increased. For Crohn's disease patients there was a corresponding increase in clinical remission. Patients with high trough levels had their dose reduced. Overall this optimisation strategy was shown to be cost-effective. Patients were then randomised to dosing over the following year based on clinical features alone or based on trough levels. There was no difference in remission between the two groups, but there was a lower rate of flare in those randomised to the trough level-based dosing.

As discussed in the Section 4.9.3.1: Infliximab for perianal fistulising disease, higher levels may be required for patients with perianal fistulae. ${ }^{586}$ Drug levels vary according to the assay used, and consensus has not yet been achieved on the optimal therapeutic ranges. A flow chart for the use of anti-TNF therapeutic drug monitoring is presented in figure 4.

\subsubsection{Primary non-response to anti-TNF therapy}

Treatment failure to anti-TNF therapy should be divided into primary non-response (failure to respond to induction therapy) and secondary loss of response. For patients with primary non-response to one anti-TNF, the likelihood that they will respond to a second is small but is dependent on the clinical 


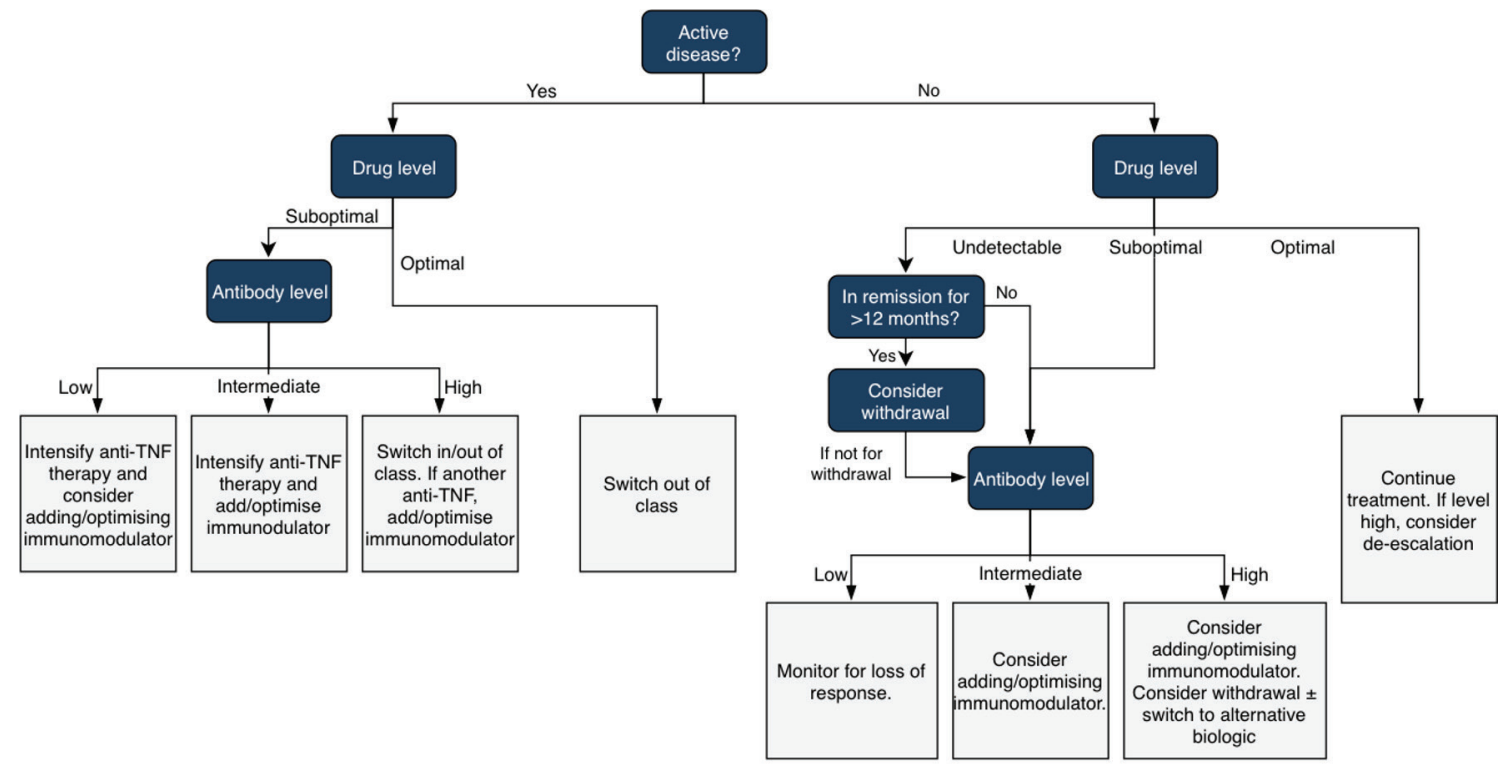

Notes:

- 'Optimal' drug levels for infliximab and adalimumab are not defined, depend on the assay used, and clinical context

- After dose optimisation, the regular use of therapeutic drug monitoring in patients in remission is not currently recommended and further evidence of cost-effectiveness is awaited

Figure 4 Anti-TNF therapeutic drug monitoring.

context. Switching to a drug that acts through a different mechanism is more likely to be successful. Measuring drug and antibody levels may have a role, with recent evidence suggesting that drug levels in primary non-responders are often lower than in responders, ${ }^{817}$ and antibody formation can be a significant factor within a few weeks of treatment initiation.

\subsubsection{Secondary loss of response to anti-TNF therapy}

Secondary loss of response to anti-TNF therapy can occur as a consequence of immune-mediated neutralising antibodies to the drug (although there are likely to be other mechanisms including non-neutralising, drug-clearing antibodies or non-immune-mediated mechanisms). Measurements of drug and antibody levels are helpful in guiding next steps. ${ }^{818}$ A retrospective analysis of 247 IBD patients with 330 loss of response events (188 to infliximab and 142 to adalimumab) showed that, following loss of response, trough concentration measurements and anti-drug antibodies could inform the likely outcome of interventions. Patients with either adequate drug concentrations (adalimumab $>4.5 \mu \mathrm{g} / \mathrm{mL}$ or infliximab $>3.8 \mu \mathrm{g} / \mathrm{mL}$ ) or positive anti-drug antibodies (adalimumab $>4 \mu \mathrm{g} / \mathrm{mL}$ equivalent or infliximab $>9 \mu \mathrm{g} / \mathrm{mL}$ equivalent) did better by switching to an alternative anti-TNF or an alternative class of drug, whereas patients with low drug concentration and negative antibodies improved with dose increase. ${ }^{819}$ A decision analytic model in Crohn's patients losing responsiveness to infliximab showed that a testing strategy was likely to be cost-effective compared with empiric changes in treatment. ${ }^{820}$ In a prospective study of IBD patients with secondary loss of response to infliximab, all of whom had a dose increase, mucosal healing occurred in half of patients and was associated with a rise in trough levels. ${ }^{821}$ In patients losing response to infliximab and manifesting with 'end of dose' recurrence of Crohn's symptoms, drug levels and anti-drug antibody levels should be checked and consideration given to starting concomitant immunomodulator therapy where this is not already being used. To increase the drug levels of infliximab by shortening the infusion interval to 6 weeks may be as effective as shortening to 4 weeks or giving a $10 \mathrm{mg} / \mathrm{kg}$ dose. ${ }^{822}$ Once remission has been recaptured over a period of 3-4 months, it may be possible to reduce the dose or stretch the intervals back out again without losing response. In a further retrospective study in 168 Crohn's patients losing response, 112 patients were treated with $10 \mathrm{mg} / \mathrm{kg} 8$-weekly and 56 with $5 \mathrm{mg} / \mathrm{kg}$ at 4 -week intervals. Sustained response was achieved in 50\% who received double dose and 39\% of those with interval shortening, OR 1.5 (95\% CI 0.8 to 2.9$).{ }^{823}$ Doubling the dose is generally more convenient and cost-effective than interval shortening.

A significant proportion of patients have detectable drug levels and a low titre of anti-drug antibodies. Management in this situation is not clear as antibodies may be transient ${ }^{824}$ and, as shown in a post-hoc analysis of the TAXIT trial, ${ }^{825}$ infliximab dose escalation can facilitate an adequate trough drug level and can result in clinical response despite the presence of anti-drug antibodies. Starting the patient on immunomodulator therapy (if they are not already on this) can abolish the antibody response and restore efficacy, particularly if combined with an increase in anti-TNF dose. ${ }^{826}$

The presence of low drug levels in the presence of high titre antibodies (and perhaps even low titre antibodies in the context of adalimumab) mandates a switch, ideally within class to an alternative anti-TNF therapy, particularly if the patient has previously shown themselves to be anti-TNF responsive

Statement 92. We suggest that treatment options for failure of initial anti-TNF therapy (increase dose, shorten dosage interval, switch to alternative anti-TNF, or switch to different drug class) may be informed by the clinical context and by measurement of serum drug and anti-drug antibody concentrations (GRADE: weak recommendation, low-quality evidence. Agreement: $97.7 \%)$ 
Statement 93. We suggest that patients with secondary loss of response to anti-TNF therapy may have serum drug and antidrug antibody concentrations measured to inform appropriate changes in treatment (GRADE: weak recommendation, moderate-quality evidence. Agreement: 97.6\%).

over a prolonged period. Of note, individuals who form antibodies to one biological therapy may be more prone to forming them to a second. ${ }^{827}$ In this context, having a low threshold for combining the second biologic with immunomodulator therapy is logical to reduce the risk of future antibody-mediated loss of response.

Many of the published studies on switching from originator to biosimilar infliximab have measured drug and anti-drug antibody concentrations before switching. ${ }^{803828}$ This may identify patients with low drug levels and/or antibodies, who probably should not switch, with a more appropriate action either to withdraw therapy (if in deep remission) or switch to a different drug class.

\subsubsection{Annual review of patients on biologics therapy}

National Institute for Health and Care Excellence (NICE) technology appraisals for biological therapies in IBD (infliximab, adalimumab, golimumab, vedolizumab and ustekinumab) recommend annual review. ${ }^{137}{ }^{829-832}$ Although there is little evidence to support this, it is sensible to assess the safety and efficacy of long-term treatment with both immunosuppressive and biological drug therapy. Patients may be having their treatment in infusion facilities or at home, and a formal clinical review is necessary to ensure that changing circumstances, adverse effects of treatment, long-term clinical remission or other factors do not make it more appropriate to withdraw treatment or substitute alternative therapy. ${ }^{816}$

\subsubsection{Drug management: vedolizumab and ustekinumab}

Vedolizumab and ustekinumab are contraindicated in patients with active TB, sepsis or opportunistic infections, including gut infections such as Clostridium difficile. Pretreatment screening should be undertaken as for anti-TNF treatment. Latent TB should be treated prior to commencing either drug. Patients should be up to date with vaccination prior to starting treatment where possible. Non-live vaccines may be administered during either drug treatment, but not live vaccines. Both drugs should be stopped if severe infection develops.

\subsubsection{Vedolizumab}

Close monitoring for evidence of progressive multifocal leucoencephalopathy (PML) in patients treated with vedolizumab has shown no cause for concern. However, patients should be monitored for and advised to report any neurological symptoms that develop because of the rare occurrence of PML in patients treated with other integrin receptor antagonists in conjunction with systemic immunosuppressive drugs.

Integrated long-term safety data (May 2009 to June 2013) showed that serious C. difficile infections, sepsis or TB occurred

Good Practice Recommendation 16. IBD patients receiving immunomodulators or biologics should have an annual review of treatment, including consideration of response and treatment continuation, optimisation or cessation (Agreement: 97.7\%).
Statement 94. We suggest that pretreatment screening and blood monitoring of therapy on vedolizumab and ustekinumab should at present follow recommendations for anti-TNF drugs due to insufficient long-term safety data at this time to recommend an alternative algorithm (GRADE: weak recommendation, very low-quality evidence. Agreement: $95.3 \%)$

in $0.6 \%$ of patients and there were no cases of PML. ${ }^{165833}$ Other studies show no increase in infection risk. ${ }^{834-836} \mathrm{~A}$ meta-analysis of 49 studies of biologics with safety data in IBD did show that there is an increase in risk of opportunistic infections that is no different between anti-TNF and anti-integrin agents. Longterm safety data for vedolizumab over 9 years are reassuring. ${ }^{163}$ The same study showed very small numbers of malignancies. ${ }^{837}$ An advantage of the gut-specific immunosuppression of vedolizumab, however, is that it does not alter the immune response to parenterally administered antigens, although it does affect the response to orally administered vaccines. ${ }^{838}$ There is still uncertainty about the mechanism of action of vedolizumab, with recent data showing that modulation of innate immunity contributes to its therapeutic efficacy. ${ }^{839}$ Subanalysis and data from the GEMINI studies have shown that the frequency of infusion related reaction (IRR) with vedolizumab is around $5 \% .^{840}$

\subsubsection{Ustekinumab}

Ustekinumab, through inhibition of IL-12 and IL-23, inhibits key molecules in cellular immunity. Although there are no head-to-head data with anti-TNF therapy, safety appears to be very good. Data from dermatological studies (where the drug has been NICE-approved for treatment of psoriasis since 2009) are also reassuring. Analyses of psoriasis registry data show that anti-TNF therapies are associated with a greater risk of serious infection (1.9-2.9/100 patient-years) compared with ustekinumab (0.93/100 patient-years) ${ }^{841}$ although at a lower dose of ustekinumab than used in Crohn's disease. A further safety review in psoriatic arthritis treatment concluded that the most common events were respiratory tract infections, nasopharyngitis, headache and injection site reactions. ${ }^{842}$ In 167 Crohn's patients failing anti-TNF therapy, treated with ustekinumab, a very similar side effect profile occurred, with no malignancy, $\mathrm{TB}$ or deaths attributed to the drug. It was noted that $11.4 \%$ developed arthralgia, ${ }^{512}$ but arthralgia was not noted to be more common on active treatment than placebo in the IM-UNITI maintenance trial. Adverse events associated with infusions in the UNITI and IM-UNITI trials occurred in 3.4\% of patients. ${ }^{506}$

\subsubsection{Extraintestinal manifestations (EIM) of IBD and vedolizumab therapy}

There has been interest in the impact of vedolizumab on patients with extraintestinal manifestations (EIMs). The OBSERVIBD GETAID cohort followed 294 patients treated with vedolizumab (173 Crohn's disease, $121 \mathrm{UC}$ ) of whom 49 (16.7\%) had EIMs at baseline (47 arthropathy, four skin, and two both joints and skin). At week 54, 44.7\% had complete remission of their arthropathy and $75 \%$ of their skin EIMs. On multivariable analysis, remission of arthropathy was associated with clinical remission of their luminal disease and with recent onset of arthropathy. However, during follow-up 13.8\% 
developed de novo arthropathy and $4.8 \%$ paradoxical skin inflammation. ${ }^{843}$

\subsubsection{Cancer and treatment with biologics}

Biologics in the treatment of IBD are not completely contraindicated in patients with prior malignancy, and decisions should be individualised. A delay of at least 2 years after successful cancer eradication is appropriate, and this should be extended to 5 years for those cancers with a high risk of late metastatic spread (including breast, malignant melanoma and renal cell carcinoma). ${ }^{844}$ Data from the New York Crohn's and Colitis Organisation for 333 IBD patients with a history of cancer followed up for 5 years did not show any differences in the rate of cancer-free survival between patients treated with anti-TNF therapy, immunomodulators, anti-TNF/thiopurine combination therapy or no immunosuppression. ${ }^{845}$ Evidence related to malignancy in relation to IBD and its treatment is reviewed in recent ECCO guidance. ${ }^{846}$

\subsubsection{Withdrawal of immunosuppression for patients in sustained remission}

\subsubsection{Stopping thiopurines}

A recent systematic review summarised the published data on thiopurine withdrawal in patients in clinical remission. ${ }^{192}$ Relapse rates were higher among patients randomised to withdrawal, and at 12 months ranged from $16.5 \%$ to $53 \%$ in Crohn's disease and from $11 \%$ to $77 \%$ in UC. Fewer studies reported longer term rates: at 5 years ranging from $63 \%$ to $85 \%$ in Crohn's disease and $43 \%$ to $65 \%$ in UC. Two studies reported on the rate of success at reintroducing thiopurines in Crohn's disease, with rates of $74 \%{ }^{194}$ and $96 \%,{ }^{847}$ with only a single study reporting this outcome in UC with successful reintroduction in $92 \%$ of cases. ${ }^{194}$ The risk of relapse should be weighed against the emerging risks of longer term thiopurine therapy, including infections and cancer. In particular, the absolute risk increase of lymphoma rises markedly with increasing age. ${ }^{848}$

\subsubsection{Stopping anti-TNF therapy}

From studies assessing outcomes of stopping therapy after at least 12 months of anti-TNF therapy, the estimated relapse rate at 1 year was $39 \%$ for Crohn's and $35 \%$ for UC/IBD-U. ${ }^{849}$ The estimated relapse rates at 2 years were 54\% for Crohn's disease and $42 \%$ for UC/IBD-U. Among patients relapsing and retreated with anti-TNF, the estimated chances of success were $88 \%$ for Crohn's disease and $76 \%$ for UC/IBD-U. Various models to predict chance of relapse have been proposed. ${ }^{192}$ The clinical factors associated with relapse, however, vary between studies. ${ }^{850}$ Younger age, smoking, longer disease duration, fistulising perianal Crohn's disease, anaemia, raised CRP and raised faecal calprotectin are linked with increased risk of relapse. Mucosal healing (and in some studies low serum drug levels) are associated with a lower risk of relapse. Patients receiving escalated dosages of anti-TNFs or for the indication of postoperative recurrence in Crohn's disease had a $>75 \%$ risk of relapse on drug withdrawal. Persistent abnormalities on MRE in Crohn's disease patients in endoscopic remission also makes relapse more

Statement 95. We suggest that IBD patients in prolonged remission on thiopurines, and who have mucosal healing, may stop the drug after discussion of risks and benefits and considering patient preference. Reintroduction if relapse occurs is usually successful (GRADE: weak recommendation, low-quality evidence. Agreement: 95.3\%).
Statement 96. We suggest that anti-TNF therapy may be withdrawn in patients with prolonged corticosteroid-free remission and mucosal healing. Retreatment in the event of relapse is usually successful, but there is insufficient evidence about which clinical factors predict relapse after withdrawal and decisions should be individualised (GRADE: weak recommendation, very low-quality evidence. Agreement: 93.2\%).

likely. ${ }^{851}$ Decisions regarding withdrawal should be taken in the context of the individual patient, their disease history and the consequences of relapse. ${ }^{852}$ Patient profiles favouring treatment withdrawal include: older patients without previous complications, surgery and with no evidence of active inflammation, those with comorbidities increasing their infection risk, those with undetectable trough drug levels and other practical reasons mitigating against continuing injections or infusions. ${ }^{853}$ There is no safe minimum period of corticosteroid-free remission, but at least 1-2 years seems reasonable. The concept of cyclical biologics use (stopping when deep remission is established and restarting in the event of pre-clinical relapse) has been introduced, but there is no evidence as yet that this is safe or cost-effective. ${ }^{853}$ All the published data so far come from prospective and retrospective cohort studies such as the STORI study. ${ }^{854}$ Data on outcomes after withdrawal of newer biologics are awaited.

\subsubsection{Monitoring following immunosuppressive and biological therapy withdrawal}

As with stopping thiopurines, faecal calprotectin rises prior to clinical relapse. ${ }^{855856}$ A study of serial faecal calprotectin, C-reactive protein, full blood count and protein electrophoresis at 3-month intervals after stopping azathioprine showed that calprotectin was the only predictor of clinical relapse in both Crohn's disease and UC. ${ }^{857}$ Serial measurement of faecal calprotectin offers a potential opportunity to escalate therapy prior to the development of symptoms, although prospective evidence to validate increasing therapy in this context is lacking. It should not be measured routinely in all patients, but targeted to those where there are significant anxiety or concerns related to stopping anti-TNF therapy.

\subsubsection{Drug management: corticosteroids \\ 5.2.8.1 Overuse of corticosteroids}

Corticosteroids are effective agents at inducing clinical remission, but do not have a role in preventing relapse. ${ }^{99858}$ Steroid dependency is generally defined as an inability to wean below $10 \mathrm{mg}$ of prednisolone or $3 \mathrm{mg}$ of budesonide within 3 months of starting, or disease flare within 3 months of stopping steroids; steroid refractory disease is active disease despite taking up to $1 \mathrm{mg} / \mathrm{kg} /$ day of prednisolone for 4 weeks. Steroid excess is considered to be two or more courses of steroid over 1 year. A recent audit found that $14.9 \%$ of IBD patients had steroid dependency or excess in the UK, more commonly in UC than Crohn's disease. ${ }^{448}$ Prolonged steroid use (often defined as continuous therapy for more than 3 months) is associated with numerous side effects including increased infection risk, osteoporosis, suppression of the hypothalamic-pituitary-adrenal axis, diabetes, weight gain and cardiovascular disease. There is evidence of increased mortality in IBD patients on long-term steroids, with a study in USA Medicare and Medicaid patients showing that prolonged steroid use (more than $3000 \mathrm{mg}$ prednisolone equivalent in 1 year) carries greater mortality (significant for Crohn's, non-significant for UC) compared with patients starting anti-TNF therapy, ${ }^{859}$ with data from the TREAT registry also showing that 
Statement 97. We suggest that patients in whom anti-TNF therapy is withdrawn should be observed for evidence of relapse. Monitoring of faecal calprotectin may be helpful in this context as levels may rise before clinical relapse occurs (GRADE: weak recommendation, low-quality evidence. Agreement: 97.9\%).
Good Practice Recommendation 17. Risk factors for osteoporosis in IBD include prolonged corticosteroid use, but general risk factors should also be screened for and corrected, including malnutrition, inflammation, smoking and lack of weight-bearing exercise (Agreement: 100\%). prednisolone use was an independent risk factor for mortality. ${ }^{516}$ In an audit excessive steroid use was avoidable in nearly half of patients, ${ }^{448}$ was more likely to be avoided if patients attended a dedicated IBD clinic, and if there was a local multidisciplinary IBD team. Steroid use in the previous year is an important disutility included in the ICHOM dataset, ${ }^{346}$ and documenting assessment of alternative therapies and discussion with the patient is important for all patients on long-term steroids.

\subsubsection{Corticosteroid-related bone disease}

Approximately $35-40 \%$ of patients with IBD suffer from osteopenia and 15\% from osteoporosis, and both Crohn's disease and UC patients have increased risk. ${ }^{860-862}$ Risk factors include uncontrolled inflammation, malabsorption (particularly in Crohn's disease), weight loss, prolonged or high-dose oral steroid use or lack of physical activity. ${ }^{863-866}$

\subsubsection{Calcium and vitamin D supplementation}

For patients who have to receive corticosteroids, other factors should be addressed that have a negative impact on bone mineral density. This includes advice to stop smoking, reduce excess alcohol intake and partake in regular muscle-building and weight-bearing exercise such as weight training or running. ${ }^{860}$ Vitamin D deficiency is common, and occurs in more than half of patients with UC and Crohn's disease in the UK and northern Europe. ${ }^{867868}$ In older people, there is evidence from a systematic review that vitamin $\mathrm{D}$ supplementation with daily dose of 700-800 IU reduces the risk of hip and non-vertebral fractures. ${ }^{869}$ A study of patients with Crohn's disease showed that treating their active disease, and supplementing vitamin D with 800 IU and 500-1000 mg calcium daily, resulted in a small increase in bone density over the next 4 years. ${ }^{870}$ Steroids reduce calcium absorption from the gut and increase urinary losses, leading to secondary hyperparathyroidism which results in bone resorption. A Cochrane review of five trials confirmed that calcium and vitamin D supplementation for all patients treated with steroids prevented bone loss from the lumbar spine and forearm. ${ }^{871}$ Calcium supplementation may increase cardiovascular risk (particularly if not accompanied by vitamin D supplementation). A Swedish long-term cohort study showed that a total (dietary and supplements) calcium intake greater than $1400 \mathrm{mg}$ daily was associated with increased all-cause mortality (HR 1.4; 95\% CI 1.17 to 1.67$).{ }^{872}$ Calcium supplements should not be given without vitamin $\mathrm{D}$, and treatment with calcium and vitamin D should not therefore continue long term unless dietary calcium intake is less than $800 \mathrm{mg}$ daily. An online calcium calculator can be used to estimate dietary calcium intake (http://www.

Statement 98. We recommend that prolonged corticosteroid therapy is harmful and should be minimised by specialist intervention and involvement with the multidisciplinary team to explore other treatment options (GRADE: strong recommendation, moderate-quality evidence. Agreement: $97.8 \%)$. cgem.ed.ac.uk/research/rheumatological/calcium-calculator/). Vitamin D should be measured and supplemented if deficient, with a high loading dose followed by maintenance.

Reduction in bone mineral density and fracture risk are worsened by oral corticosteroid use, and the greatest bone loss occurs in the first 6 months of treatment. ${ }^{873} 874$ Before starting oral steroids, patients should have their fracture risk assessed as shown in figure 5. For patients aged over 40, the Fracture Risk Assessment Tool (FRAX) can be used to assess the 10 year probability of a major osteoporotic fracture (http:/www.shef.ac.uk/FRAX/). Patients with a high FRAX score (score $\geq 20 \%$ major fracture and $\geq 3 \%$ hip fracture), those aged under 40 with risk factors or those receiving prolonged (more than 3 months) or repeated courses of oral corticosteroids should have their bone mineral density assessed by bone densitometry. ${ }^{861875}$ Note that FRAX does not discriminate past versus current corticosteroid use, nor does it stratify risk according to doses beyond $7.5 \mathrm{mg}$, and risk should be adjusted accordingly. ${ }^{876} 877$ Patients on long-term steroids should have a repeat bone densitometry at 1 year, and if stable, repeated at 2-3-year intervals, but if declining, repeated annually. Women and men aged 70 or more, with past fragility fracture, taking high-dose steroids $(7.5 \mathrm{mg}$ or more prednisolone-equivalent), or at high risk based on FRAX score, should be considered for bone-protective therapy at the start of steroid treatment. ${ }^{878}$ Alendronate or risedronate should be used, unless contra-indicated or not tolerated, in which case intravenous zoledronate or teriparatide should be given. For women of childbearing age requiring bone-protective therapy, if they are taking effective contraception (or not sexually active), oral bisphosphonates are the first-line therapy followed by teriparatide if oral bisphosphonates cannot be used. ${ }^{878}$ Denusomab and high-dose intravenous bisphosphonates should not be used in this situation due to lack of data on the risk of fetal harm should an unplanned pregnancy occur. There is a lack of safety data on use of denosumab with immunosuppressive therapy, and on all osteoporosis therapies during pregnancy, other than calcium and vitamin D. ${ }^{878}$

Bisphosphonate or denosumab therapy are associated with the rare adverse effects of osteonecrosis of the jaw or atypical femoral fractures. Current UK guidance for the prevention and treatment of osteoporosis recommends dental examination with preventative dentistry in those with dental disease or other risk factors (eg, use of corticosteroids or smoking). ${ }^{877}$ These guidelines also encourage patients to maintain good oral hygiene and regular check-ups with reporting of new oral symptoms (eg, dental mobility, pain or

Statement 99 . We recommend that all patients receiving a course of corticosteroids for a disease flare should receive an intake of $800-1000 \mathrm{mg} /$ day calcium and $800 \mathrm{IU} /$ day vitamin D (GRADE: strong recommendation, very low-quality evidence). This can be achieved by administration of oral calcium and vitamin D supplements while on corticosteroids, or vitamin D only if dietary calcium intake is adequate. Lifestyle modification advice including regular physical exercise and smoking cessation should also be provided (Agreement: 90.9\%). 
Statement 100 . We recommend that patients starting corticosteroids should be assessed for risk of osteoporosis. Those at high risk should be started on bisphosphonate therapy at the onset of corticosteroid therapy (GRADE: strong recommendation, high-quality evidence), after ensuring adequate calcium intake and supplementing vitamin D (Agreement: 90.7\%).

swelling) while on therapy. ${ }^{877}$ Treatment review is recommended to consider stopping bisphosphonate after 5 years (after 3 years for zolendronate) unless patients are aged over 70, have previous hip/vertebral fracture, sustain a fracture during treatment or are continuing $\geq 7.5 \mathrm{mg}$ prednisolone, in which case treatment may be continued. ${ }^{877}$ If treatment is discontinued fracture risk should be assessed after a new fracture, or if no new fracture should be assessed after 18 months to 3 years. ${ }^{877}$

\subsubsection{Corticosteroid-related adrenal suppression}

Corticosteroid withdrawal syndrome can present with weakness, fatigue, loss of appetite, weight loss, nausea and vomiting, diarrhoea and abdominal pain, and so can mimic the underlying disease. It is under-recognised, occurring in about half of patients tested immediately after withdrawal of medium- or high-dose prednisolone used for long periods. ${ }^{879}$ Symptoms may be mild,

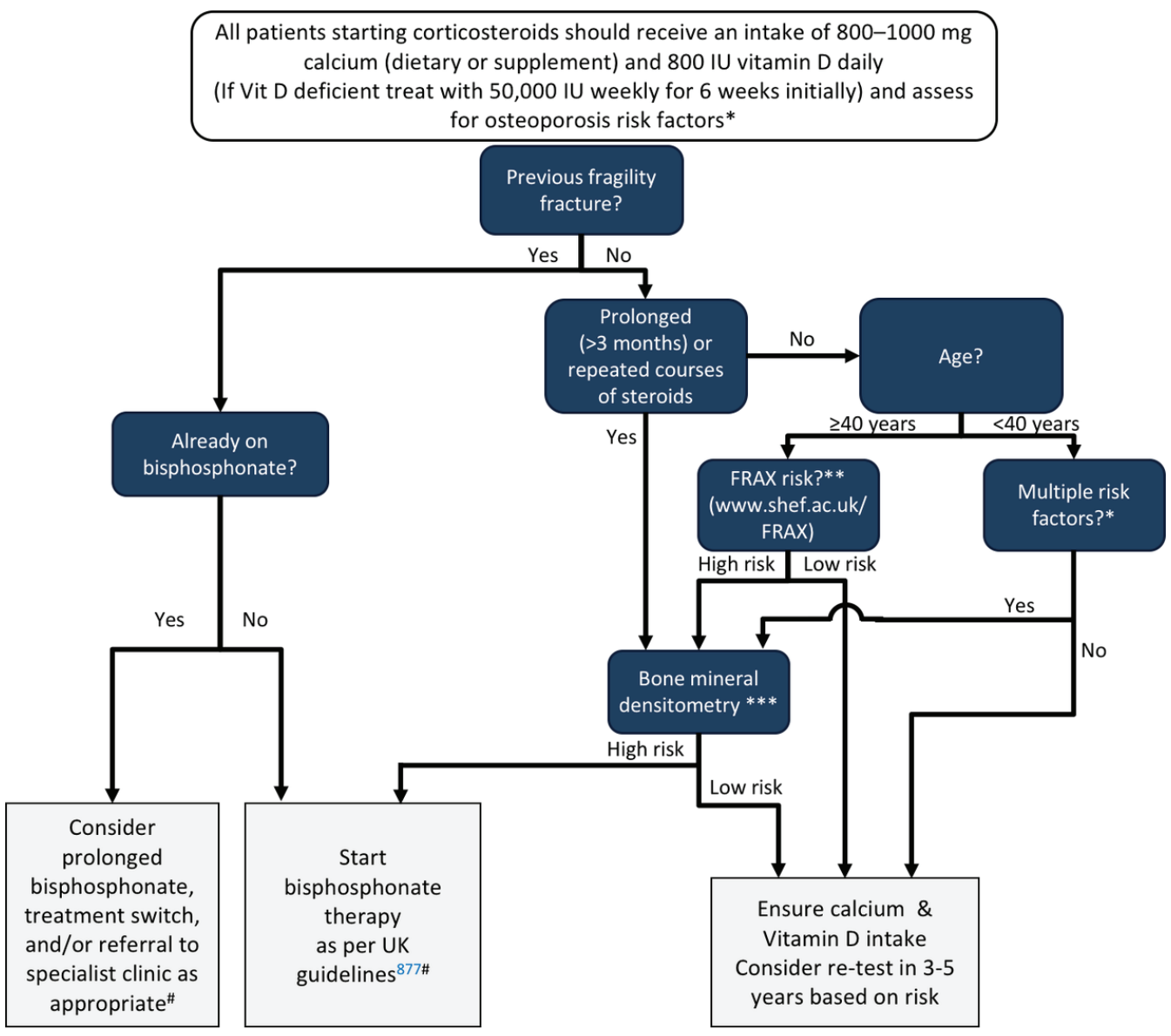

* Assess and modify risk factors wherever possible: Prolonged or high dose steroids, Uncontrolled inflammation, Weight loss and malabsorption, Lack of physical activity esp. weight-bearing, alcohol excess, smoking

** FRAX assumes an average dose of 2.5-7.5 mg/day prednisolone or equivalent, thus may underestimate fracture risk in those taking higher doses, or overestimate risk in those taking lower doses. Average adjustments over all ages in postmenopausal women and men $\geq 50$ years are shown below ${ }^{877}$

\begin{tabular}{|cccc|}
\hline & $\begin{array}{c}\text { Prednisolone } \\
\text { equivalent }(\mathrm{mg} / \text { day) }\end{array}$ & $\begin{array}{c}\text { Average adjustment: hip } \\
\text { fracture probability }\end{array}$ & $\begin{array}{c}\text { Average adjustment: } \\
\text { major osteoporotic } \\
\text { fracture probability }\end{array}$ \\
\hline Low & $<2.5$ & \% risk $\times 0.65$ & $\%$ risk $\times 0.8$ \\
Medium & $2.5-7.5$ & None & None \\
High & $\geq 7.5$ & \% risk $\times 1.2$ & $\%$ risk $\times 1.15$ \\
\hline
\end{tabular}

*** Arrange bone mineral densitometry as soon as possible. As fracture risk increases within 3 months of starting corticosteroids, if testing cannot be undertaken within 1 month, treatment to prevent osteoporosis should be commenced in high risk patients. Patients on long-term steroids should have a repeat bone densitometry at 1 year, and if stable, repeated at two to three-year intervals, but if declining, repeated annually. High-risk threshold for intervention is T score -1.5, (or a FRAX 10 year $\geq 20 \%$ risk for major osteoporotic fracture).

\# Intravenous zoledronic acid given annually may be used first line where there is evidence of malabsorption or increased risk of gastrointestinal side effect from oral bisphosphonate use. Zolendronic acid should also be considered where patients have developed a fracture despite oral bisphosphonate. For patients intolerant of bisphosphonates or where contraindicated, denosumab or teriparatide are alternative agents

Figure 5 Osteoporosis prevention and management in IBD. 
Good Practice Recommendation 18. Patients receiving prolonged courses of corticosteroids are at risk of adrenal suppression and should have a tapering course if stopping. They should be warned about possible steroid withdrawal syndrome, including non-specific symptoms such as weakness, nausea and arthralgia (Agreement: 97.8\%).

with decompensation only when exposed to stressful situations, or can be severe. Stimulation tests (such as the ACTH test or short synacthen test) are abnormal in 46-100\% immediately after stopping, but still abnormal in $26-49 \%$ after 1 week, and in a few patients remain abnormal 6-22 months later. ${ }^{880}$ Neither previous prednisolone dose nor duration are good predictors of the problem, which will occur with rectal-administered as well as oral steroids, and can occur after as little as 4 weeks of treatment. Steroid tapering does not completely prevent its occurrence. There is no optimal test to confirm adrenal suppression in this situation. Steroid dose and duration should be minimised where possible, with slow taper, and awareness when suggestive symptoms occur. Slow tapering of budesonide is not required as there is little or no suppression of the HPA axis.

\subsubsection{Other metabolic complications of corticosteroids}

Before starting oral corticosteroid therapy for 3 months or longer or repeating oral steroids within 6 months of a previous course, record height, weight, blood pressure, full blood count, fasting plasma glucose or HbA1c and lipids. Blood pressure and BMI should be measured at every clinic visit. For patients with dyslipidaemia or a raised cardiovascular risk at initiation, repeat a lipid profile 1 month after initiation and then every 3-6 months. Monitor fasting glucose or HbA1c every 3 months. An annual examination by an ophthalmologist is required for patients with symptoms of cataracts, a personal or family history of open angle glaucoma, diabetes mellitus, high myopia or connective tissue disease (particularly rheumatoid arthritis). Mood disturbance such as depression or sleep disturbance should be questioned at every clinic visit. Steroids may exacerbate the potassium-lowering effects of thiazide or loop diuretics, and potassium should be monitored. ${ }^{875}$ Avascular necrosis of the femoral head is a serious complication of prolonged steroid use, and if hip pain develops should be considered, and if suspected urgent MRI is recommended along with cessation of steroids. Patients on long-term steroids should be vaccinated as for those on immunosuppressive therapy (see Section 5.2.1.3: Common Disease Considerations, Immunosuppressive therapy, Vaccination).

\subsubsection{Prevention of Pneumocystis jirovecii infection}

Prophylaxis for Pneumocystis jirovecii using co-trimoxazole has been shown to reduce incidence by $91 \%$ in a meta-analysis of studies in transplant recipients and haematological malignancy. ${ }^{881}$ In the Spanish registry ENEIDA study of 571 patients treated with rescue therapy, two developed $P$. jirovecii pneumonia (PJP) and one died. Both had received double immunosuppression (corticosteroids and azathioprine) and had not received prophylaxis, which was given to all receiving triple immunosuppression. The risk relates as much to use of corticosteroids as to other immunosuppressive drugs. ${ }^{234}$ A study from the USA showed a low incidence of PJP in IBD patients despite low use of prophylaxis. There were three cases identified among 937 patients with 6066 patient-years follow-up with wide confidence intervals around the risk related to each class of immunosuppressant. ${ }^{882}$ Overall risk is low. PJP prophylaxis should perhaps be restricted
Good Practice Recommendation 19. Patients on prolonged corticosteroids should have blood pressure, glycaemic control and serum potassium monitored. They should receive vaccinations as for those on immunomodulatory therapy (Agreement: 82.6\%).

to those on triple therapy taking $\geq 20 \mathrm{mg}$ prednisolone, particularly those with other risk factors (age, frailty, comorbidity). The optimal dose regimen is unclear, but in adults co-trimoxazole $960 \mathrm{mg}$ three times per week or $480 \mathrm{mg}$ daily are widely used. Stevens-Johnson syndrome is a rare but devastating complication of co-trimoxazole and should be discussed with patients when offering co-trimoxazole therapy.

\subsection{Diet and IBD}

Dietary patterns and food consumption vary hugely across the world and the diet is very different in countries with a high prevalence of IBD compared with countries with a low prevalence. From epidemiological evidence, when people migrate from a country with a low prevalence of IBD to a country with a high prevalence they are at increased risk of developing IBD, thus incriminating environmental changes. Dietary components that have been associated with an increase in the risk of or relapse of IBD include cereals, dietary fibre, sugar, fat, fruit, vegetables and protein. ${ }^{883-887}$ However, no one dietary component is consistently blamed. Thus, until strong scientific evidence supports a defined diet, patients with IBD should be advised to eat a varied diet to meet their energy and nutrient requirements, including dietary fibre. The diet (box 8) should be based on local healthy eating guidelines and include a wide variety of fruit and vegetables, cereals, grains, nuts and seeds, protein-rich foods with a moderation/reduction of high fat, particularly animal fat, high sugar and processed (cured, salted or smoked) meats. Patients with stricturing Crohn's disease may need to alter their intake of dietary fibre and fibrous foods for symptomatic management of strictures and may need supplementation with enteral or parenteral nutrition to achieve their energy and nutritional requirements. ${ }^{424436}$ Management of short gut or intestinal failure due to Crohn's disease is covered well elsewhere. ${ }^{888}$

\subsubsection{Nutritional assessment and monitoring}

Malnutrition is common in IBD and comprises undernutrition and overnutrition. Up to $85 \%$ of patients with IBD have undernutrition. ${ }^{889}$ Unintentional weight loss and low BMI are common in patients with newly diagnosed IBD and during disease relapse. The causes are multifactorial and include suboptimal nutritional intake, alterations in energy/nutrient requirements and metabolism, malabsorption, excessive gastrointestinal losses and medication. Overnutrition occurs in $15-40 \%$ of patients with IBD and may in part be due to improved management of IBD and the obesity epidemic. ${ }^{890} 891$ Thus, nutritional assessment in patients with IBD is crucial to identify and appropriately manage patients with or at risk of malnutrition.

Statement 101. We suggest that adult IBD patients on triple immunosuppression and using more than $20 \mathrm{mg}$ prednisolone may be offered prophylactic antibiotics for Pneumocystis jirovecii (co-trimoxazole oral $960 \mathrm{mg}$ three times weekly or $480 \mathrm{mg}$ daily) (GRADE: weak recommendation, very low-quality evidence. Agreement: 85.7\%). 
Good Practice Recommendation 20. IBD patients should be encouraged to eat a varied diet that meets their energy, macro- and micronutrient requirements. All who are at risk of malnutrition should have dietitian or nutrition team review, and where nutritional requirements cannot be met, supplementation with enteral or parenteral nutrition are indicated (Agreement: $100 \%)$.

There are limited data on the frequency of routine nutritional assessment in IBD, although inpatients seem to be much more frequently assessed ${ }^{892}$ than outpatients. ${ }^{436} 893$

Standard nutritional assessments should assess BMI, recent unintentional weight loss and the nature of disease. However, deficits in body composition, muscle strength and serum micronutrients are common in IBD, and BMI alone cannot detect these. An increase in fat mass and decrease in fat-free mass are often reported and increased intra-abdominal fat (visceral fat) is linked to increased cardiovascular risk, increased surgical complications and hospitalisation. ${ }^{890}$ A more detailed assessment of nutritional status should also include measurements of body composition (waist circumference (a proxy for visceral adiposity ${ }^{894}$ ), tricep skinfold thickness, mid arm muscle circumference), muscle (hand-grip) strength and micronutrient status (dietary intake and serum measurements). ${ }^{895}$

\subsubsection{Blood tests for specific nutritional deficiencies}

Measurement of macronutrient and micronutrient status in IBD is important for ongoing monitoring in patients who have or are at risk of malnutrition; however, interpretation of results can be challenging. Some micronutrients are influenced by disease activity so serum levels should consider C-reactive protein (CRP) when interpreting these micronutrients. A reliable clinical interpretation can be made only if the CRP is $<20 \mathrm{mg} / \mathrm{L}$ (plasma zinc), $<10 \mathrm{mg} / \mathrm{L}$ (plasma selenium, vitamin A and vitamin $\mathrm{D}$ ) or $<5 \mathrm{mg} / \mathrm{L}$ (vitamin B6 and vitamin C). ${ }^{896}$ Magnesium deficiency has been reported to occur in $13-88 \%$ of patients with IBD, ${ }^{897}$ but serum magnesium is not an accurate measurement of magnesium status because less than $1 \%$ of magnesium stores are in the blood. The remainder is in bone, soft tissue and muscle. ${ }^{898}$ Magnesium deficiency in IBD is likely to be caused by increased gastrointestinal losses. Symptoms of magnesium deficiency include abdominal cramps, impaired healing, fatigue and bone pain. Magnesium supplementation can be given orally or intravenously, although oral supplementation can worsen diarrhoea. Up to a third of patients with IBD fail to meet the recommended dietary intake for calcium and calcium supplementation should be considered alongside vitamin D supplementation (see Section 5.2.8.3: Common Disease Considerations, Calcium and vitamin D supplementation). Plasma potassium levels should be monitored and supplemented as required. There are existing ECCO guidelines for monitoring and management of iron, vitamin B12 and folate deficiency. ${ }^{899}$ General advice about iron replacement is shown in box 9 .

\subsubsection{Vitamin D}

In a multicultural UK IBD cohort, the prevalence of vitamin D deficiency $(<50 \mathrm{nmol} / \mathrm{L})$ was $66 \%$ in Crohn's disease and $69 \%$ in UC, and severe vitamin D deficiency was $27 \%$ in Crohn's disease and 36\% in UC. ${ }^{867}$ Median vitamin D levels were lower in black and Asian ethnic groups relative to white subjects, and

\section{Box 8 Practical dietary advice in IBD}

In general:

- Some dietary components (ie, cereals, dietary fibre, sugar, fat, fruit, vegetables and protein) have been associated with $\mathrm{IBD}$; however, a relapse or ongoing symptoms cannot be attributed to one dietary component

- Non-evidence based self-directed exclusion diets are to be discouraged as they can lead to limited diet quality and nutrient deficiency

- All IBD patients should be advised to eat a varied diet to meet energy and nutrient requirements, including dietary fibre. The diet should be based on local healthy eating guidelines and include a wide variety of fruit and vegetables, cereals, grains, nuts and seeds, protein-rich foods with a moderation/reduction of high fat, particularly animal fat, high sugar and processed meats

- Patients with stricturing Crohn's disease: consider limiting dietary fibre and fibrous foods. Supplementation with enteral or parenteral nutrition may be required to achieve energy and nutritional requirements

- Patients with functional bowel symptoms (in remission/ mildly active disease): consider giving dietary advice as for irritable bowel syndrome (eg, low FODMAP diet)

\section{Diet with an ileostomy*}

- Initial dietary advice: small frequent, nutrient dense meals/ snacks and oral nutritional supplements where necessary

- Sodium: to prevent dehydration, sprinkle extra salt onto meals; add 0.5-1 teaspoon per day

- Potassium: increase intake if serum potassium is low (potassium-rich foods suitable for an ileostomy include bananas, potatoes and potato crisps, spinach, fish, poultry, lean red meat, sweet potato, avocado)

- Fibre: a high fibre intake can increase loose stools, flatulence and bloating

- Thickening output: bananas, pasta, rice, white bread, mashed potato, marshmallows or jelly

- Fluids: avoid chronic dehydration and advise 2-2.5 litres of fluids per day, more during hot weather or exercise. Check urinary sodium to detect dehydration. Be cautious with high intakes of hypotonic (eg, tea, water) and hypertonic (eg, fruit juice) drinks as these can increase stoma output and increase dehydration. Encourage isotonic drinks (eg, Dioralyte, sports drinks) and if ileostomy output exceeds one litre per day recommend oral rehydration solution: 1 litre tap water with six level teaspoons glucose, one level teaspoon salt, half teaspoon sodium bicarbonate or sodium citrate with or without flavouring

- 1-2 tablets (2-4 mg) loperamide half hr before meals.

- Avoid stoma blockages: recommend chewing food thoroughly and advise caution with fruit and vegetable skins, sweetcorn and celery and avoid nuts (smooth nut butters are fine).

- Vitamin B12 monitoring.

- An ileostomy pathway based on patient education for monitoring fluid balance post discharge reduces readmission rates for dehydration. ${ }^{1287}$

* Ileostomy diet advice does not apply to patients with short gut, jejunostomy or high-output ileostomy. 
Good Practice Recommendation 21. Patients with IBD should have an assessment of their general nutritional status and screening for evidence of recent weight loss and/or assessment of malnutrition risk at each clinic appointment and on hospital admissions (Agreement: 95.7\%).

IBD-related surgery was associated with vitamin D deficiency in the Crohn's disease group (OR 2.9). A recent Norwegian study reported the prevalence of vitamin D deficiency (25-OH-D concentrations $<50 \mathrm{nmol} / \mathrm{L}$ ) to be $53 \%$ in Crohn's disease and $44 \%$ in UC. ${ }^{868}$ Vitamin D levels in IBD patients are lower than in the general population. ${ }^{900901}$ Latitude did not influence the association between IBD and vitamin D. ${ }^{901}$

In Crohn's disease patients, vitamin D concentrations were inversely associated with disease activity measured by the Harvey Bradshaw index, and in UC vitamin D deficiency was associated with elevated calprotectin $>100 \mathrm{mg} / \mathrm{kg}$. Vitamin $\mathrm{D}$ deficiency is associated with low bone mineral density. ${ }^{902}$ Vitamin D is important in some immune-mediated diseases although its role in IBD inflammation is not fully elucidated. ${ }^{903}$ Retrospective studies have shown an association between higher levels of vitamin D and remission rates, ${ }^{904}$ and durability of response to anti-TNF therapy. ${ }^{905906}$ It is unclear whether this association is due to confounding by increased disease activity associated with lower vitamin D (although controlled for in these studies).

Limited data exist on the impact of vitamin D supplementation on clinical disease course in IBD. A small double-blind randomised controlled trial of 108 patients with Crohn's disease treated with 1200 IU daily of oral vitamin D3 or placebo for 1 year just failed to reach significance with $13 \%$ in the intervention group versus $29 \%$ in the placebo group experiencing a clinical relapse $(p=0.06) .{ }^{907}$ Small uncontrolled studies show that high-dose vitamin D improves clinical indices in Crohn's disease. ${ }^{908} 909$ Vitamin D status should be routinely measured in IBD and deficiency should be corrected using oral supplementation, with evidence that this reduces risk of bone disease; but as yet the impact of vitamin D on inflammation in IBD and its impact on disease course, and the optimal vitamin D level as a goal of treatment is poorly understood.

\subsubsection{Dietary treatment of functional symptoms in IBD}

At least a third of patients with inactive IBD have coexisting functional bowel symptoms (eg, abdominal pain, bloating, increased flatulence, diarrhoea and/or constipation) and these symptoms may be mistaken for active IBD. ${ }^{910}$ Objective markers of disease activity (histological and inflammatory markers (eg, faecal calprotectin, CRP)) alongside clinical symptom assessment help to distinguish between functional bowel symptoms and active IBD. Identification of functional bowel symptoms in inactive IBD is important to avoid

Statement 102. We recommend that IBD patients who are malnourished or at risk of malnutrition should have relevant screening blood tests to assess for macronutrient and micronutrient deficiencies. This may include measurement of iron stores, vitamin B12, folate, vitamins A, C, D and E, potassium, calcium, magnesium, phosphate, zinc and selenium (GRADE: strong recommendation, very low-quality evidence. Agreement: 93.6\%).

\section{Box 9 Iron deficiency anaemia in IBD}

- A third of patients with active IBD have iron deficiency anaemia

- Anaemia causes fatigue, affects quality of life and delays recovery

- As systemic inflammation inhibits absorption of iron, iron tablets should not be used in those with active disease and, in patients with inactive disease, no more than $100 \mathrm{mg}$ elemental iron should be taken daily ${ }^{899}$

- Ferritin levels up to $100 \mu \mathrm{g} / \mathrm{L}$ in the presence of inflammation may still reflect iron deficiency. ${ }^{899}$ Measurement of transferrin saturation may therefore be helpful

- Consider other causes of anaemia (eg, folate deficiency, B12 deficiency or bone marrow depression) in patients with IBD

- Intravenous iron should be used first line in patients intolerant of oral iron who have active IBD and moderate to severe anaemia $(\mathrm{Hb}<100 \mathrm{~g} / \mathrm{L})$, or those who need erythropoietin $^{899}$

- Iron absorption may be improved by once daily or alternate daily oral dosing ${ }^{1288}$

unnecessary and potentially harmful treatment strategies. In contrast, active disease should be excluded before establishing that symptoms are functional in nature. From a dietary perspective, identification of dietary triggers is useful but it is often difficult due to diet complexity and a delay in symptom generation following food ingestion. Many patients with IBD alter their diet in an effort to control their symptoms, whether during periods of active disease or during remission. ${ }^{911}$ Indeed, these self-induced dietary restrictions may be detrimental to patients' nutritional status, therefore supervision by a dietitian may be required. A diet low in fermentable oligosaccharide, disaccharide, monosaccharide and polyols (FODMAP) has become a recognised successful management strategy for functional bowel disorders like IBS. In IBD, 56\% of patients who had previously received low FODMAP dietary advice reported overall symptom improvement. ${ }^{912}$ Furthermore, in a dietary re-challenge study, FODMAPs (specifically fructans) increased functional bowel symptoms in patients with quiescent IBD. ${ }^{913}$ In a prospective study of a low FODMAP diet in 88 IBD patients, $78 \%$ of patients reported satisfactory relief from their functional bowel symptoms at follow-up compared with only $16 \%$ at baseline. ${ }^{914}$ Mechanisms by which food-related functional symptoms in IBD develop, and which dietary interventions are effective and safe for the management of functional symptoms in inactive IBD, remain unclear.

\subsection{Preoperative optimisation}

Despite improvements in medical therapy, the rate of surgery in Crohn's disease after 5-10 years ranges from approximately 20\%-30\%. . $^{536} 915$ Postoperative complications are also more common in patients undergoing IBD surgery than operations

Statement 103. We suggest that vitamin D levels should be measured and deficiency corrected in Crohn's disease and ulcerative colitis (GRADE: weak recommendation, very low-quality evidence. Agreement: 86.7\%). 
Statement 104. We suggest that a low FODMAP diet may be used to treat functional bowel symptoms in IBD patients (GRADE: weak recommendation, low-quality evidence. Agreement: 84.4\%).

for other conditions due to the active inflammation, emergency setting, complications and drug treatment. ${ }^{916}$

\subsubsection{Preoperative nutritional status}

Malnutrition and nutrient deficiencies are common in IBD. ${ }^{917918}$ Severe malnutrition (BMI $<18.5 \mathrm{~kg} / \mathrm{m}^{2}$ and recent weight loss $>10 \%$ body weight) is associated with a higher risk of postoperative complications, particularly intra-abdominal sepsis ${ }^{919}$ and increased mortality. ${ }^{920}$ Poor preoperative nutritional status has been identified as an independent risk factor for postoperative intra-abdominal septic complications (OR $6.23,95 \%$ CI 1.75 to 22.52 ) in multivariate analysis. ${ }^{921}$ Thus, all IBD patients undergoing surgery should be assessed for risk of malnutrition. Elective surgery should ideally be postponed until malnutrition is treated. However, in an emergency situation this may not be feasible, and should be considered in the nature of operation undertaken to minimise risk of complications.

\subsubsection{Evidence for preoperative nutritional support}

There are few prospective studies of preoperative nutrition and no prospective randomised trials with a non-nutrition control group. A meta-analysis of pre-surgical nutritional support in gastrointestinal surgery patients found that the provision of $500-1000 \mathrm{kcal}$ of an immune enhancing oral nutritional supplement plus usual food significantly reduced post-surgical complications. $^{922}$ A more recent systematic review of preoperative nutrition in Crohn's disease included 14 original studies, but only five prospective studies including one randomised trial. ${ }^{923}$ Five studies showed significantly reduced complications and infectious episodes in patients receiving preoperative nutritional therapy. Significant heterogeneity was reported between studies in terms of the nutritional supplement used and timing of nutrition. There is sufficient evidence to propose delaying surgery when possible to allow a multimodal approach to management including nutrition, steroid weaning and management of any abscesses.

The European Society of Parenteral and Enteral Nutrition (ESPEN) recommends preoperative nutritional support for 7-10 days in patients who are undergoing major gastrointestinal surgery and are mildly malnourished. ${ }^{924}$ A longer duration is recommended for severely malnourished patients even if it delays surgery. ${ }^{924}$ If oral nutritional supplements are not tolerated, then enteral nutrition should be considered and parenteral nutrition should only be used when nutritional targets cannot be delivered by the enteral route. ${ }^{436} 924$

Statement 105. We recommend that IBD patients should have assessment and optimisation of their physical condition prior to elective surgery. This should include appropriate imaging to determine disease extent and complications; radiological drainage of abscesses and treatment of sepsis; correction of anaemia; treatment of malnutrition and physical mobilisation (GRADE: strong recommendation, very low-quality evidence. Agreement: 97.3\%).
Statement 106. We recommend that prior to surgery all IBD patients should have their nutritional status assessed and if at risk of malnutrition should receive nutritional support (oral nutritional supplements or enteral or parenteral nutrition if required) (GRADE: strong recommendation, very low-quality evidence. Agreement: 97.3\%).

\subsubsection{Preoperative serum albumin}

Hypoalbuminaemia (albumin $<30 \mathrm{~g} / \mathrm{L}$ ), as a reflection of significant inflammation or secondary to malabsorption, is frequently associated with severe malnutrition although is not in itself a marker of nutritional status. ${ }^{919}$ This level of hypoalbuminaemia is associated with a higher risk of postoperative intra-abdominal sepsis. ${ }^{925}$ The evidence to support the use of intravenous albumin is weak ${ }^{919} 925$ and correction of hypoalbuminaemia hinges on treatment of underlying sepsis and control of inflammation. Nutritional support alone is very unlikely to restore low albumin levels to normal while sepsis and uncontrolled inflammation persist, but feeding is an important supportive measure. ${ }^{926} 927$

\subsubsection{Preoperative anaemia}

Preoperative anaemia $(\mathrm{Hb}<130 \mathrm{~g} / \mathrm{L}$ in men and $<120 \mathrm{~g} / \mathrm{L}$ in women) increases the risk of postoperative intra-abdominal sepsis, correction of which is associated with improvement in outcomes including risk of intestinal obstruction and haemorrhage, anastomotic leak, postoperative perforation, pulmonary oedema and septic complications such as pneumonia and wound infection. ${ }^{925} 928$ Perioperative red cell transfusion in patients undergoing ileocaecal resection has been shown to be associated with an increased risk of postoperative complications including an anastomotic leak, ileus, intra-abdominal abscess, wound dehiscence and thrombotic events. ${ }^{929}$ Anaemia should be recognised and treated early, preferably with oral or intravenous iron and other haematinics as needed, and avoiding blood transfusion where possible.

\subsubsection{Preoperative corticosteroids}

Patients undergoing IBD surgery while on corticosteroids have an increased risk of postoperative infectious complications and anastomotic leaks. ${ }^{930-932}$ There is some evidence that risks are greater for those taking high-dose steroids $(40 \mathrm{mg}$ prednisolone or more). ${ }^{932933}$ A comparison of prednisolone doses greater than $20 \mathrm{mg}$ versus $20 \mathrm{mg}$ or less did not show a significant difference in risk of infections. ${ }^{931}$ Use of more than $\geq 15 \mathrm{mg}$ oral corticosteroid in UC patients within 30 days of IPAA surgery, or more than $\geq 20 \mathrm{mg}$ in the setting of proctocolectomy, is associated with increased risk of complications. ${ }^{934} 935$ IBD patients having elective surgery should have their corticosteroids stopped if possible, or brought to as low a dose that can be managed without deterioration.

Patients who are on corticosteroids at the time of their IBD surgery should be given intravenous hydrocortisone in equivalent dosage until they can resume oral prednisolone. ${ }^{930}$ Prednisolone $5 \mathrm{mg}$ is equivalent to hydrocortisone $20 \mathrm{mg}$ or

Statement 107. We suggest that patients with penetrating or stricturing Crohn's disease, or those who are malnourished, may benefit from exclusive or partial enteral nutrition for at least 6 weeks preoperatively (GRADE: weak recommendation, very low-quality evidence. Agreement: 97.2\%). 
Statement 108. We recommend that prior to elective surgery for Crohn's disease or ulcerative colitis, corticosteroids should be stopped or dose minimised wherever possible to reduce risk of postoperative complications (GRADE: strong recommendation, low-quality evidence. Agreement: 94.6\%).

methylprednisolone $4 \mathrm{mg}$. There is no value increasing steroid dosage to cover stress in the perioperative period, as shown in a randomised trial in IBD surgery ${ }^{936}$ and case series. ${ }^{937}$ Anaesthetists will generally give a single steroid dose prior to induction (such as dexamethasone $4 \mathrm{mg}$ intravenous or intramuscular) for those taking more than $5 \mathrm{mg}$ prednisolone. ${ }^{938}$ Patients who are on physiological corticosteroid replacement because of disorders of the hypothalamic pituitary axis (such as oral hydrocortisone $20 \mathrm{mg}$ mane, $10 \mathrm{mg}$ nocte) should receive supplementary doses in the perioperative period. ${ }^{939}$ For patients who have had complete resection of active disease, it is important to avoid inappropriate prolongation of steroids after surgery, and there is virtue in standardised steroid-taper protocols in the postoperative period, dependent on the dose and duration of steroids preoperatively (table 11).

\subsubsection{Preoperative thiopurines}

With one exception, ${ }^{940}$ the literature on the use of immunosuppressive therapy (thiopurines and methotrexate) leading up to surgery does not describe an association with an increased risk of postoperative complications. ${ }^{932} 933941$

\subsubsection{Preoperative anti-TNF therapy}

The risk of surgical complications for Crohn's patients taking anti-TNF therapy during the perioperative period has been assessed in many small observational studies. The majority of systematic reviews and meta-analyses conclude that there is a small increase in risk of infectious complications, 257932 942-945 although one systematic review found no difference. ${ }^{946}$ This risk is less than that for corticosteroid use, ${ }^{947}$ and anti-TNF therapy alone does not justify the formation of a diverting stoma. Operative risk should be based on other risk factors including presence of fistulae, abscess, low albumin, anaemia and corticosteroid use in addition to anti-TNF therapy. ${ }^{930}$ There is evidence in Crohn's disease that risk is related to anti-TNF drug levels. ${ }^{948}$ If clinically appropriate, cessation should be 6-8 weeks before surgery for infliximab and 4 weeks for adalimumab, but evidence to support this is lacking. If necessary, treatment should be restarted soon after the patient is well enough to be discharged from hospital. For UC, compared with Crohn's disease there are fewer data on which to assess risk of anti-TNF therapy and studies have methodological limitations, but two meta-analyses conclude that the postoperative risk is not increased overall. ${ }^{944} 949$ Anti-TNF therapy for Crohn's patients should therefore be discontinued where possible prior to elective surgery. If not appropriate to stop, then timing of injections should be arranged to have as long a gap as possible prior to the operation. Stopping anti-TNF therapy prior to UC surgery is less critical.

Statement 109. We recommend that IBD patients who have been on oral corticosteroids for more than 4 weeks prior to surgery should receive an equivalent intravenous dose of hydrocortisone while nil by mouth in the perioperative period (GRADE: strong recommendation, moderate-quality evidence. Agreement: 100\%).
In patients having proctocolectomy, corticosteroids are associated with an increased risk of impaired wound healing and need for re-operation. ${ }^{935}$ There is no evidence of increased complication risk for patients taking immunomodulators (mercaptopurine, ciclosporin) who have ileoanal pouch surgery ${ }^{950}$ or IBD surgery generally. ${ }^{941}$ A two-stage IPAA procedure carries more risk than a three-stage procedure if patients are on anti-TNF therapy. ${ }^{951}$ Pouch surgery is complex with inherent risks, and it is prudent to conduct a controlled withdrawal of both corticosteroids and anti-TNF as part of a staged approach to pouch formation, which should always be done in an elective setting.

\subsubsection{Summary of recommendations for operative optimisation}

In elective surgery IBD patients should have their physical condition assessed and optimised prior to surgery. This should include assessment of comorbidities; imaging or endoscopy to document disease extent; drainage of abscesses and treatment of sepsis; assessment and correction of nutritional deficiencies; and stopping corticosteroids and biologics where possible. All IBD patients undergoing surgery should follow an enhanced recovery (ERAS) protocol. ${ }^{436} 924$ A checklist is shown in box 10.

\subsection{Faecal calprotectin}

\subsubsection{Faecal calprotectin in the differential diagnosis of IBD versus} IBS

Faecal calprotectin is a sensitive and specific marker of intestinal inflammation and is a useful non-invasive method to investigate patients with chronic gastrointestinal symptoms in low-risk groups for malignancy, when infection and coeliac disease have been excluded as the cause and symptoms are suspected to be either due to IBD or IBS (figure 6). A normal level has a very high negative predictive value for IBD. ${ }^{952-954}$ By contrast, the positive predictive value of levels above the assay reference level (normal defined as $<50 \mu \mathrm{g} / \mathrm{g}$ stool) is low, and it is recommended that a higher threshold is used to trigger colonoscopy, which improves the positive predictive value with little reduction in the negative predictive value. ${ }^{955956}$ Due to the variety of calprotectin assays in commercial use, the exact cut-off prompting colonoscopy should be determined locally, but it is generally between 100 and $250 \mu \mathrm{g} / \mathrm{g}$ stool and should be monitored to ensure appropriate specificity and sensitivity. Used in this way, faecal calprotectin can be used as a cost-effective measure ${ }^{957}$ to prevent unnecessary colonoscopy in those where IBD is extremely unlikely. ${ }^{958}$

Faecal calprotectin is generally raised in patients with bloody diarrhoea and patients with this presenting feature will need flexible sigmoidoscopy regardless of the calprotectin result. In acute diarrhoea due to infection, calprotectin is also likely to be raised and will not discriminate between IBD and gastroenteritis, thus stool culture and/or flexible sigmoidoscopy or colonoscopy are the appropriate diagnostic investigations. While there is limited evidence to support routine repeat testing, an evaluation of the York Faecal Calprotectin Care Pathway found that, in 243 patients with a faecal calprotectin $\geq 100 \mu \mathrm{g} / \mathrm{g}$ after a median of 18 (IQR 14-29) days, repeat testing identified a reduction in $53 \%$ of patients, either to $<100 \mu \mathrm{g} / \mathrm{g}$ or, if initially $>250 \mu \mathrm{g} / \mathrm{g}$, into the $100-250 \mu \mathrm{g} / \mathrm{g}$ range. ${ }^{959}$

Faecal calprotectin is not sensitive enough in the exclusion of advanced colorectal adenoma or colorectal carcinoma, ${ }^{960} 961$ and so in patients with rectal bleeding and abdominal pain, change in bowel habit, weight loss, or iron-deficiency anaemia, cancer pathway referral should be strongly considered ${ }^{962}$ and calprotectin is not appropriate. In primary care, symptoms of change 
Table 11 Postoperative prednisolone tapering

\begin{tabular}{lll}
\hline Preoperative prednisolone dosage & Initial dose after surgery & Taper regimen \\
\hline $5-20 \mathrm{mg}$ daily & Same dose as before surgery & Reduce by $5 \mathrm{mg}$ every 3 days and stop \\
$>20 \mathrm{mg}$ daily for $<3$ weeks & Same dose as before surgery & Reduce by $5 \mathrm{mg}$ every 3 days and stop \\
$>20 \mathrm{mg}$ daily for $>3$ weeks at any time during the past 6 months & Same dose as before surgery & $\begin{array}{l}\text { Reduce by } 10 \mathrm{mg} \text { weekly until } 20 \mathrm{mg} \text {, then reduce by } 5 \mathrm{mg} \text { weekly until } \\
5 \mathrm{mg} \text {, then after } 2 \text { weeks give } 2.5 \mathrm{mg} \text { for } 2 \text { weeks then stop }\end{array}$ \\
\hline
\end{tabular}

Adapted from Lightner et al..$^{938}$

in bowel habit with rectal bleeding and abnormal haematology in patients under 50 years are strongly predictive of IBD or colorectal cancer, but generally do not discriminate between the two. ${ }^{963}$ IBD is significantly more common in younger age groups, so referral will generally be to medical gastroenterologists. However, if there are reasons to suspect malignancy, patients should be referred via a suspected cancer pathway, although both need rapid colonoscopy (figure 6). ${ }^{962}$

\subsubsection{Faecal calprotectin in the assessment of disease activity in known IBD}

Faecal calprotectin is a useful surrogate marker of inflammatory activity in IBD. ${ }^{964}$ It correlates well with endoscopic inflammation in UC $^{965}$ and in Crohn's disease. ${ }^{966}$ If it is unclear whether new symptoms represent a relapse or other causes, particularly in Crohn's disease, then calprotectin is useful to confirm active inflammation and can be a non-invasive alternative to flexible sigmoidoscopy or colonoscopy and cross-sectional imaging. If relapsing disease is clinically obvious, then measurement is not necessary.

A recent meta-analysis including data for 2822 IBD patients and 298 controls found that a calprotectin cut-off of $50 \mu \mathrm{g} / \mathrm{g}$ had the best sensitivity $(90.6 \%)$ to detect endoscopically active disease, with specificity performing best (78.2\%) at levels $>100 \mu \mathrm{g} / \mathrm{g} .{ }^{967}$ A separate meta-analysis has shown that a threshold of $250 \mu \mathrm{g} / \mathrm{g}$ provides better specificity of $82 \%$ than thresholds of $100 \mu \mathrm{g} / \mathrm{g}$ and $50 \mu \mathrm{g} / \mathrm{g}$ (specificity of $66 \%$ and $60 \%$, respectively) in differentiating active IBD from remission. ${ }^{968}$ However, a faecal calprotectin of $250 \mu \mathrm{g} / \mathrm{g}$ had a relatively lower sensitivity of $80 \%$ compared with a sensitivity of $84 \%$ and $92 \%$ at cut-offs of $100 \mu \mathrm{g} / \mathrm{g}$ and $50 \mu \mathrm{g} / \mathrm{g}$, respectively.

\subsubsection{Use of faecal calprotectin to inform therapy changes}

There is increasing evidence of the benefits of mucosal healing in reducing future relapse rate. ${ }^{5052969970}$ Meta-analyses have shown that faecal calprotectin can discriminate between active and inactive IBD, although accuracy is greater for UC than Crohn's disease. ${ }^{968971}$ Studies in UC patients in clinical remission show that calprotectin correlates with endoscopic inflammation, ${ }^{972}$ and for those with both clinical and endoscopic remission, correlates with histological inflammation. ${ }^{973}$ A further study showed that calprotectin levels were a better predictor of relapse over the next year than the presence of histological inflammation. ${ }^{974}$

The information about mucosal inflammation provided by faecal calprotectin levels is a practical means to target increases in drug dosage, and studies are now starting to investigate the value this has in improving long-term outcomes. In UC, patients with

Statement 110. We recommend that patients with ulcerative colitis should not be considered for pouch surgery while taking corticosteroids (GRADE: strong recommendation, very low-quality evidence. Agreement: 94.1\%). clinically inactive disease but raised calprotectin can have levels reduced to those associated with lower risk of relapse by increases in dose. ${ }^{67}$ In Crohn's disease the open-label randomised CALM study has shown that calprotectin levels below $250 \mu \mathrm{g} / \mathrm{g}$ stool, CDAI $<150$, CRP $<5 \mathrm{mg} / \mathrm{L}$ and no prednisolone can be used as a target, with dose escalation of adalimumab and azathioprine at intervals until these levels are reached. ${ }^{360}$ At 1 year the 'treat to target' group achieved the primary end-point of a colonoscopic CDEIS score $<4$ without deep ulcers in $45.9 \%$, with only $30.3 \%$ of the control group achieving this $(p=0.010)$. Calprotectin may also be useful in predicting the likelihood of future relapse in order to decide who can withdraw therapies. In the STORI study, patients stopping anti-TNF with mucosal healing and a calprotectin $\geq 300 \mu \mathrm{g} / \mathrm{g}$ had a relapse rate of $30 \%$, but those with both mucosal healing and a lower calprotectin had a relapse rate of between $10 \%$ and 20\%. ${ }^{854} 975$ Serial measurement over time can give a more accurate prediction of the likelihood of relapse. ${ }^{976}$

\subsubsection{Collection of faecal calprotectin samples}

There remain practical issues around the use of faecal calprotectin. These include variability between different assays, variability in levels in different stool samples from one patient during 1 day, lack of stability for samples kept at room temperature for more than a few days, and variation in calprotectin levels related to the interval between stools being passed. ${ }^{977}{ }^{978}$ It is therefore recommended that the first stool passed in the morning is routinely used for sampling, and that this is stored for no more than 3 days at room temperature before analysis.

\subsection{Lower GI endoscopy in IBD patients}

\subsubsection{Flexible sigmoidoscopy and colonoscopy in acute colitis}

In the 1970s and 1980s it was recommended that colonoscopy be avoided in severe colitis because of the risk of toxic megacolon or perforation, ${ }^{979-981}$ or be limited to a flexible sigmoidoscopy

\section{Box 10 IBD operative checklist}

\section{Before admission:}

- Notify physicians of planned admission date if elective surgery or notify of emergency admission

- Optimise nutrition in weeks prior to elective surgery

- Smoking cessation education and support including from general practitioner and community support services

- Stoma nurse counselling (where relevant) and appropriate psychological support including peer support

- Agree and implement plan regarding medical therapy: minimisation of steroid use; dose optimisation of immunomodulators and biologics prior to and after surgery

Postoperative during inpatient stay:

- Dietary assessment and nutritional plan

- Tail prednisolone as per table 11.

- Consider medical prophylaxis 
Statement 111. We recommend that, for patients aged 16-40 presenting in primary care with chronic diarrhoea and symptoms that may be consistent with either IBD or IBS, faecal calprotectin is a useful screening tool with a high negative predictive value. If significantly elevated, patients should have an infective cause excluded and be referred for further investigation (GRADE: strong recommendation, moderate-quality evidence. Agreement: 97.9\%).

without bowel preparation. ${ }^{982}$ Colonoscopy equipment and technique has changed radically since then, and more recent data suggest that it can be performed safely and provide useful information in acute severe colitis. ${ }^{983}$ Endoscopic assessment with a
Statement 112. We suggest that, in IBD patients where it is unclear if symptoms are due to ongoing inflammation or other non-inflammatory causes (such as bile acid malabsorption, functional bowel disorder or short bowel), faecal calprotectin measurement may be used to provide evidence of mucosal inflammation (GRADE: weak recommendation, low-quality evidence. Agreement: 97.8\%).

limited unprepared sigmoidoscopy and biopsies with minimal air insufflation can be performed safely by an experienced endoscopist. ${ }^{224}$ In a study of colonoscopy in IBD, complications were no more likely in severe colitis than less active disease, nor did

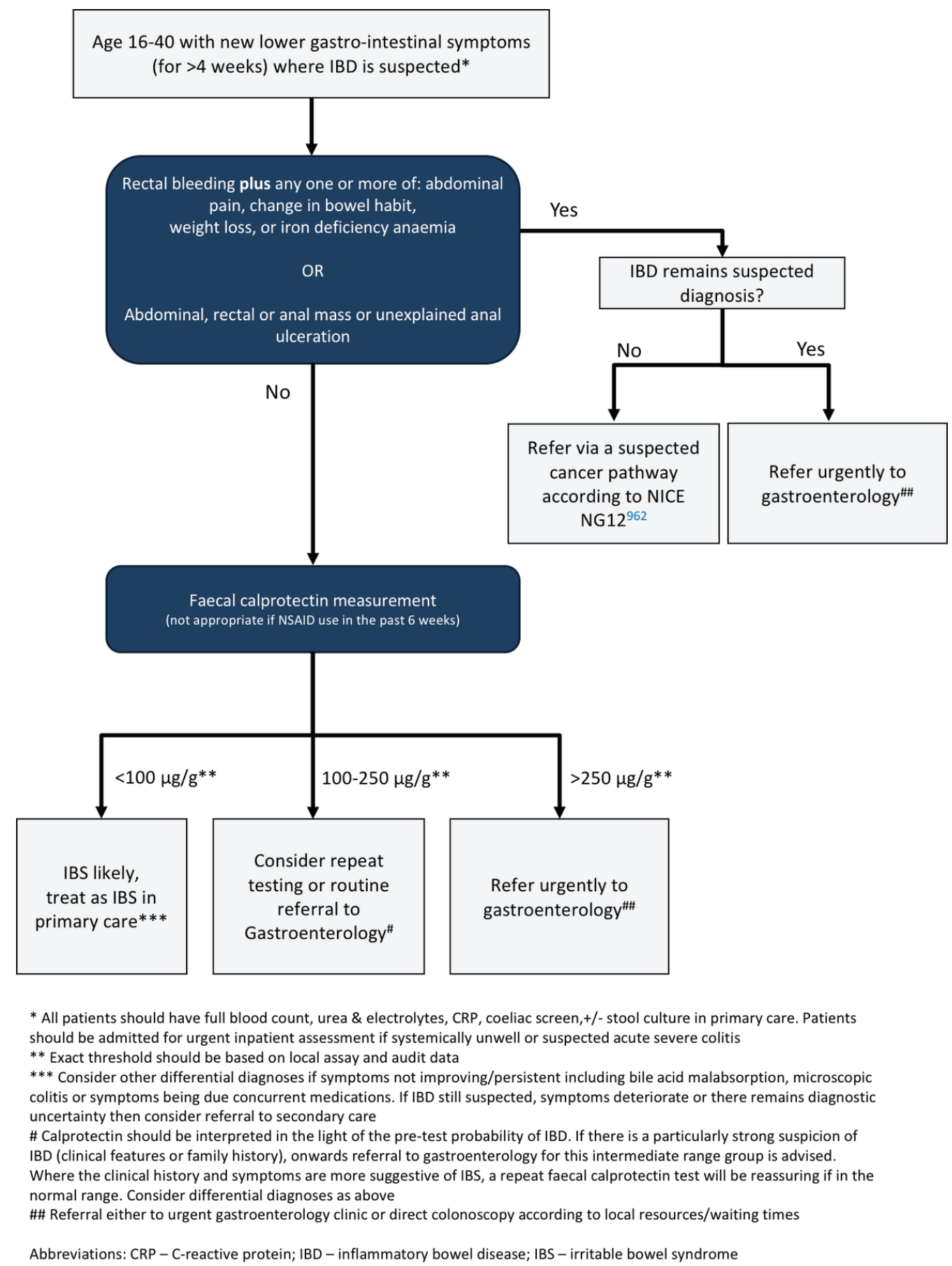

Figure 6 Use of faecal calprotectin in primary care. 
Statement 113. We suggest that faecal calprotectin is a validated biomarker for endoscopic and histological disease activity. It may therefore be a useful non-invasive parameter to inform decisions on treatment escalation or de-escalation (GRADE: weak recommendation, moderate-quality evidence. Agreement: 100\%).

tolerance of the procedure relate to disease activity. ${ }^{984}$ Retroflexion in the rectum is not recommended in the presence of active colitis due to risk of perforation. In acute severe colitis the inflamed area of rectum and colon are generally free of stool, so it is reasonable to avoid bowel preparation.

\subsubsection{Bowel preparation for colonoscopy}

High-quality preparation is desirable to allow optimal colonic mucosal visualisation and to facilitate chromo-endoscopy. There are limited studies examining the effectiveness of bowel cleansing in IBD. A prospective observational study in 429 IBD patients showed poorer bowel cleansing quality in comparison to patients attending for investigation of abdominal pain. ${ }^{985}$ In a study of 100 IBD patients with age- and sex-matched controls, disease activity was not associated with poorer quality bowel preparation according to the Boston Bowel Preparation Scale. ${ }^{986}$ However, questionnaire assessment during bowel preparation demonstrated that a higher visceral sensitivity index, anxiety and a higher Harvey Bradshaw index was associated with increased abdominal pain and nausea, which in turn led to greater anxiety immediately prior to colonoscopy. ${ }^{986}$ IBD patients also experience high embarrassment and burden from the bowel cleansing process, and are more likely to find colonoscopy painful. ${ }^{987}$ Patients may experience an increase in abdominal symptoms suggestive of a flare within 1 month following colonoscopy, ${ }^{983}$ but no definite correlation to bowel preparation has been reported. As aphthous ulceration has been associated with use of sodium phosphate, ${ }^{988}$ PEG-based bowel cleansing agents are commonly used in IBD. Low-volume regimens are better tolerated and bowel cleansing is non-inferior to high-volume PEG regimens. ${ }^{989}$

\subsection{Drug treatment adherence}

There is no gold standard definition of treatment non-adherence, but patient self-reporting is simple and reasonably accurate. ${ }^{990}$ Non-adherence is common, occurring in at least a third of IBD patients, ${ }^{991}$ most frequently for those on 5-ASA drugs. ${ }^{992}$ Although lower rates are reported with immunosuppressive drug use, ${ }^{993} 994$ even patients on infliximab have non-adherence rates of $17 \%$ and for adalimumab this could be as high as $29 \% .{ }^{995}$

The impact of non-adherence is significant, with increased relapse rates ${ }^{996}$ and generally worse clinical outcomes. ${ }^{997}$

Studies have identified numerous factors associated with IBD medication non-adherence. Although there is some heterogeneity across studies, the factors that are most consistently associated with non-adherence include psychological distress, patient beliefs (particularly about the efficacy of the drug or necessity of taking it) and discordance between the health professional and patient. ${ }^{991} 995998999$ Practical barriers to adherence (eg, cost, complex treatment regimens, inconvenience, busy lifestyle, forgetfulness) constitute a major issue for adherence, although patient perception greatly affects what is perceived as a barrier. ${ }^{998}$

\subsubsection{Strategies to improve adherence}

There are few intervention studies evaluating methods of improving non-adherence in IBD. One study evaluated education sessions ${ }^{1000}$
Statement 114 . We recommend that IBD patients should be routinely asked about medication adherence because non-adherence is common and results in worse outcomes. Risk factors include young age, psychological distress, patient beliefs and discordance between patient and health professional GRADE: strong recommendation, moderate-quality evidence. Agreement: 100\%).

with a non-significant improvement in adherence. Trials using multicomponent intervention ${ }^{1001} 1002$ are more successful in improving adherence, with techniques combining education sessions with practical aids. These include regimen simplification, medication reminder charts, weekly or daily electronic pill-box organisers with alarms, or mobile phone alarms. ${ }^{1003}$ Web-based interventions are also valuable including education about disease and how to manage flares, monitoring using a traffic-light system and access to advice from a health professional by email or phone. ${ }^{1004}$ Although psychological factors are a significant influence on adherence, ${ }^{1005}$ there are no intervention studies to date.

\subsection{Smoking}

All IBD patients should be asked about cigarette smoking. Those who smoke should be advised of the widespread harm to health, and should be advised to stop. They should be offered referral to a smoking cessation service, where they should be offered behavioural therapy (eg, cognitive behavioural therapy) in combination with pharmacotherapy (nicotine replacement, bupropion or varenicline). ${ }^{1006}$ Bupropion and varenicline should not be given to those under 18 and pregnant women should be advised about risks and benefits of nicotine replacement therapy. Without support, there is a less than 10\% likelihood of longterm abstinence in smokers attempting to stop, but these interventions increase success rates substantially. ${ }^{10071008}$

\subsubsection{Smoking and Crohn's disease}

Cigarette smoking is more common in patients diagnosed with Crohn's disease. ${ }^{1009}$ The increase in smoking is more pronounced in those diagnosed at an older age, with one USA study showing a prevalence of smoking of $47 \%$ in those diagnosed over age 40 compared with $27 \%$ in the background population. ${ }^{1010}$ Continued smoking results in a worse disease course compared with those who quit or never smokers, ${ }^{1009} 1011$ a higher risk of surgery and worse outcomes after surgery. A meta-analysis showed a 2.5 -fold increase in risk of repeat surgery and a twofold increased risk of clinical recurrence. ${ }^{637}$ It is of note that smoking makes surgical complications more common after colorectal surgery for any indication. ${ }^{1012}{ }^{1013}$ Passive smoking ${ }^{1014}$ and light smoking (less than 10 per day) are just as bad as heavy smoking. ${ }^{1015}$ The adverse effects of smoking are more pronounced in women than in men with Crohn's disease. ${ }^{1016}$

Efforts to help Crohn's disease patients quit smoking are therefore important, with evidence that setting up a smoking cessation service is cost-effective with costs paid for by reduced disease management costs. ${ }^{1017}$ Despite this, there is poor awareness among patients of the benefits of quitting ${ }^{10181019}$ and measures to

Statement 115. We recommend that IBD patients at risk of non-adherence should be offered a variety of means to improve adherence, such as regimen simplification, reminder aids and additional support and education (GRADE: strong recommendation, low-quality evidence. Agreement: 97.8\%). 
Statement 116. We recommend that smokers with Crohn's disease should be encouraged to stop, as smokers have a higher risk of disease flare, a higher incidence of surgery and a higher risk of postoperative recurrent disease (GRADE: strong recommendation, low-quality evidence. Agreement: 100\%).

help patients quit are underused. ${ }^{1020}$ Benefits of quitting accrue at any stage in the course of Crohn's disease. ${ }^{1021}$ When active measures are employed, significant numbers can be helped to stop. The TABACROHN study showed $31 \%$ of 408 patients quit initially, with $23 \%$ still abstinent after 18 months follow-up. ${ }^{1022}$

\subsubsection{Smoking and UC}

$\mathrm{UC}$ is more common in non-smokers and is more likely to arise in those who have recently quit smoking. ${ }^{1011} 1023$ The highest risk period for disease onset is in the first $2-5$ years after stopping. ${ }^{1024}$ Ex-smokers present later in life than never-smokers. ${ }^{10251026} \mathrm{UC}$ patients who smoke have reduced colectomy rates, less primary sclerosing cholangitis and less back-wash ileitis than neversmokers. ${ }^{1014}$ The same study showed higher cigarette consumption was associated with less extensive disease and reduced need for therapy. UC patients who quit smoking have a significantly worse disease course than those who continue, with more corticosteroid and azathioprine use and increased hospitalisation rates. ${ }^{1014} 1027$ Interestingly, a large study showed that smoking does not reduce the risk of pouchitis after ileal pouch-anal anastomosis for UC, neither smoking at the time of operation nor later. ${ }^{1028}$ Ex-smokers with refractory UC who resumed smoking (mean 8.6 cigarettes daily) subsequently had high rates of corticosteroid-free remission. ${ }^{1029}$ In spite of the benefits of smoking in UC, every effort should be made to encourage patients to stop. For patients who do plan to stop, a plan to mitigate the risk of worsening disease by an increase in their treatment should be discussed. Trials of transdermal nicotine therapy in the 1990s showed at best modest benefit in active UC, ${ }^{10301031}$ but maintenance trials were negative, ${ }^{1032}$ and small trials of enema therapy were shown to be associated with inability to retain the enema in $30 \%$, and side effects of nausea, light-headedness and tremor. ${ }^{1033}$ There are no trials of nicotine gum use in UC, but case reports show effectiveness in ex-smokers with refractory UC. ${ }^{1034} 1035$ Electronic cigarettes are now widely used as a cigarette substitute and, by means of a small vaporiser, provide a similar experience to smoking, with nicotine and taste and throat sensations akin to tobacco smoke. There is very little evidence of the effect of e-cigarettes on UC, although a case report surprisingly shows no benefit despite comparable amounts of nicotine. ${ }^{1036}$ The use of nicotine gum to control UC should only be recommended in refractory disease and as an alternative to resumption of smoking, with full discussion of its addictive nature.

\subsection{Psychology and stress}

A study using the UK Health Improvement Network (THIN) showed that patients with depression had a significantly

Statement 117. Ulcerative colitis patients who continue to smoke cigarettes should be encouraged to stop. There is an increased risk of flare after stopping, and patients should be made aware of this. We suggest that they are informed that an increase in medication may be required to control their disease (GRADE: weak recommendation, very low-quality evidence. Agreement: 95.7\%).
Statement 118. We suggest that in patients with IBD, psychological therapies including cognitive behavioural therapy, hypnotherapy and mindfulness meditation may be offered to interested patients, particularly those with psychological symptoms, as an adjunctive therapy to improve symptom control and quality of life (GRADE: weak recommendation, very low-quality evidence. Agreement: 91.1\%).

greater risk of developing Crohn's disease (adjusted HR 2.11, 95\% CI 1.65 to 2.70 ) and UC (adjusted HR 2.23, 95\% CI 1.92 to 2.60$).{ }^{1037}$ In this study treatment with selective serotonin reuptake inhibitors (SSRI) and tricyclic antidepressants (TCA) were protective against Crohn's disease, whereas mirtazapine, serotonin and norepinephrine reuptake inhibitors, SSRI, serotonin modulators and TCA were protective for UC.

\subsubsection{Treatment of anxiety and depression in IBD}

Anxiety and depression are common in IBD and are associated with poorer outcomes including the need for hospitalisation. ${ }^{1038-1040} \mathrm{~A}$ high proportion of patients with IBD have co-existent symptoms of IBS. ${ }^{1041} 1042$ Stress can promote a flare of IBD. ${ }^{1043}$ High quality studies of pharmacological intervention for anxiety or depression are limited and this is an area of research need. Reports of psychological intervention (psychotherapy, patient education and relaxation techniques) have demonstrated a reduction in healthcare utilisation with psychotherapy, ${ }^{1044}$ and relaxation techniques can reduce anxiety, pain and stress levels while improving quality of life and mood. ${ }^{1045}$ However, a Cochrane Systematic Review in 2011 revealed high levels of bias within studies and no benefit in health-related quality of life, emotional status or disease remission in adults, with limited benefit noted in adolescents. ${ }^{1046}$ Conflicting outcomes have been published from studies of small patient numbers regarding the impact of cognitive behavioural therapy on psychological symptoms in IBD. ${ }^{1047-1050}$ Limited studies on the therapeutic benefit of mindfulness suggest it may be a useful adjunct to pharmacological therapy to improve psychological symptoms in IBD, improve quality of life and to facilitate coping with symptoms during a disease flare. ${ }^{1051-1053}$ Gut directed hypnotherapy has an established evidence base in IBS, and though it may be useful in symptomatic control in IBD and delay flares in UC, further research is necessary in this area. ${ }^{1054} 1055$

\subsection{Pain and fatigue in IBD}

Pain is a common symptom in IBD and may be present in patients with and without evidence of clinical disease activity. ${ }^{1056} 1057$ Pain in IBD adversely affects quality of life. ${ }^{1058-1060}$ It is more common in females and in those experiencing stress, anxiety and depression. ${ }^{10611062}$ In Crohn's disease, pain is more common in smokers and those receiving steroids and antidepressants ${ }^{1063}$ and narcotics. ${ }^{1064}$ When inflammation, stricturing disease, abscesses, uncontrolled inflammation or adhesional causes of pain have been excluded, other factors should be considered. In addition to psychosocial factors, causes of pain may include co-existing irritable bowel syndrome, ${ }^{910} 10651066$ visceral hypersensitivity (which may be mediated by microscopic inflammation, including the presence of mast cells), ${ }^{10671068}$ fibromyalgia ${ }^{1069}$ and bacterial overgrowth. ${ }^{632}$

\subsubsection{Psychological therapy for unexplained pain in IBD}

Psychological interventions, especially cognitive behavioural therapy, may have a positive impact on depression and improve quality of life in IBD. ${ }^{1070}$ Due to overlap between diagnoses of IBD and IBS with visceral hypersensitivity, psychological interventions 
Good Practice Recommendation 22. Patients with IBD and pain should be investigated for stricturing disease, abscesses or uncontrolled inflammation. In the absence of an obvious cause of pain, other factors should be considered including adhesions, visceral hypersensitivity, functional bowel disorder or dysmotility, depression and/or anxiety, sleep disturbance, stress and psychosocial factors (Agreement: 95.7\%).

for pain in these conditions may also be efficacious in IBD patients. Relaxation training may improve chronic pain in UC. ${ }^{1045} 1071$

\subsubsection{Opioid medication use in IBD}

Opioid medications have analgesic and anti-motility properties. They are more likely to be prescribed to IBD patients than to matched controls. ${ }^{1072}$ Risk factors for use include female gender, multiple surgeries, severity of pain, higher clinical disease activity, a history of depression or anxiety and polypharmacy, particularly with neuropsychiatric drugs. ${ }^{1073} 1074$ Patients with sustained poor quality of life have a higher risk of subsequent opioid use and a decreased time to first opioid prescription. ${ }^{1075}$ Use of narcotics correlates with corticosteroid use in IBD. ${ }^{1076} \mathrm{~A}$ Canadian point prevalence study showed that opioid prescribing was highest in the first month following IBD diagnosis where $11 \%$ of patients received this class of drug. ${ }^{1077}$ Prescription was more common in females and in Crohn's disease relative to UC. Patients with IBD were more likely to become heavy opioid users (defined as a dose exceeding $50 \mathrm{mg}$ of morphine or equivalent per day for at least 30 consecutive days) than age-matched controls. Use of narcotics in both Crohn's disease and UC is associated with increased prevalence of depressive symptoms, ${ }^{1078}$ a higher risk of serious infection in IBD $^{516}$ and increased mortality. ${ }^{5161077}$ Historical studies show an association of opioid prescription with development of toxic megacolon in fulminant colitis. 10791080

\subsubsection{Fatigue in IBD}

Fatigue is an increasingly recognised symptom affecting patients with IBD and many other chronic inflammatory disorders. Research into IBD-related fatigue has increased in recent years. ${ }^{1081}$ IBD patients describe a spectrum of symptoms including tiredness, lethargy and lack of energy that do not subside with rest. ${ }^{1082}$ Due to a lack of understanding regarding the aetiology and complexity of individual experience of fatigue, many healthcare professionals identify the symptom as of importance but describe difficulty understanding or conceptualising fatigue, thus struggle to assess the severity of fatigue and describe frustration at not being able to adequately improve the symptom for patients. ${ }^{1082}$ Approximately 50\% of patients with IBD report fatigue at the time of diagnosis, ${ }^{1083}$ and in cohort studies the prevalence of fatigue is between $40 \%$ and $72 \%$ of IBD patients. ${ }^{1084-1088}$ Fatigue is associated with poor health-related quality of life, ${ }^{1089}$ and related factors include poor sleep quality and mental illness. ${ }^{1084}$ Disability and depression associated with fatigue is equally prevalent between UC

Statement 119. We suggest that psychological interventions may be useful for IBD patients with pain where no physical cause can be found, and may be discussed and offered as adjunctive therapy (GRADE: weak recommendation, very low-quality evidence. Agreement: 93.5\%).
Good Practice Recommendation 23. Long-term opioid use is associated with poor outcomes in IBD and should be discouraged. Investigation for causes of pain, use of alternative non-opioid drugs and psychological support should be considered (Agreement: 100\%).

and Crohn's disease. ${ }^{1089}$ Patients describe a negative impact on social and emotional well-being and a limiting effect on the ability to gain employment. ${ }^{1090}$ Studies identify active disease or history of surgical resection in Crohn's disease as a predictor of fatigue. ${ }^{1089} 1091$ However, fatigue remains very common in patients with inactive disease with a prevalence around 30-50\%. ${ }^{1084}$ 1086-1088 1091-1093

\subsubsection{Investigations and treatment in IBD patients with fatigue}

A questionnaire study of 631 patients in Europe, North America and Asia Pacific showed that daily fatigue was reported by $53 \%$ of IBD patients with anaemia. ${ }^{1094}$ Anaemia in IBD can be multifactorial including poor oral intake, malabsorption, chronic blood loss or due to chronic inflammation. Vitamin B12 and iron therapy are easily administered treatments that may correct anaemia in IBD patients. A Canadian population-based study found iron deficiency in the absence of anaemia did not contribute to fatigue. ${ }^{1095}$ However, a recent European study identified iron deficiency as a risk factor for fatigue (OR 2.5, 95\% CI 1.2 to 5.1). ${ }^{1096}$ Muscle fatigue has been associated with low serum vitamin D and magnesium. ${ }^{1097}$ Other contributing factors should also be considered including pain, sleep disturbance, alcohol misuse and emotional stress. Medications should be reviewed for those that may contribute to fatigue. Corticosteroids have been demonstrated as a determinant of fatigue, ${ }^{1085}$ although this may be a reflection of disease severity as opposed to a medication side effect. Avoidance of steroids and cessation of immunomodulatory therapy in Crohn's disease were predictors of improved physical and cognitive fatigue, respectively, in a longitudinal study. ${ }^{1084}$ Recent data have demonstrated higher circulating frequencies of memory T-cells in fatigued patients with IBD. ${ }^{1098}$ Whole blood stimulation demonstrated higher production of the Th1 cytokines TNF-alpha and IFN-gamma. Anti-TNF therapy with either infliximab or adalimumab has been shown to reduce the symptoms of fatigue. ${ }^{1099-1101}$

\subsubsection{Non-pharmacological therapy for fatigue in IBD}

A randomised controlled trial of solution-focused therapy for fatigue in quiescent IBD for 3 months showed a reduction in fatigue for up to 3 months following completion of therapy. ${ }^{1102}$ However, the effect was lost by 6 months post therapy. A further randomised controlled trial of 45 Crohn's disease patients compared professionally-led stress management versus self-directed self-management versus conventional therapy. After eight sessions, a non- significant trend to reduced tiredness was observed. ${ }^{1103}$ A longitudinal study has shown a positive effect of regular exercise on physical fatigue in IBD. ${ }^{1084}$

Good Practice Recommendation 24. IBD patients should be asked about symptoms of fatigue, as it is common and often not reported. Fatigue does not necessarily correlate with disease activity and can result in significantly worse quality of life (Agreement: 89.1\%). 
Good Practice Recommendation 25. IBD patients experiencing fatigue should be investigated for subclinical disease activity and other potentially modifiable factors considered such as sleep pattern, medication side effects, anaemia, iron deficiency, electrolyte disturbance, thyroid dysfunction, vitamin D and B12 deficiency and psychological symptoms (Agreement: 93.5\%).

\subsection{IBD-associated arthropathy}

\subsubsection{Treatment of type 1 arthropathy}

The natural history and clinical features of IBD-related arthropathies are described in the 2016 ECCO guidelines on extraintestinal manifestations of IBDe. ${ }^{1104}$ In patients with type one peripheral arthropathy, (joint pain with swelling or effusion, often asymmetric, affecting less than five joints, mainly weight-bearing lower limb joints) ${ }^{1105}$, inflammation is usually associated with flares of IBD, and responds well to treatment of the underlying disease. Local injection of corticosteroids may be required if symptoms don't resolve rapidly. A small proportion will go on to have more persistent problems, which may require sulfasalazine, methotrexate, or anti-TNF therapy.

\subsubsection{Treatment of type two arthropathy}

Type two arthropathy (more than five joints, symmetrical distribution and mainly upper limbs) is usually independent of gut inflammation, and symptoms are more persistent. Referral to a rheumatologist is helpful for consideration of immunomodulator or biological therapy, and it is important to discriminate between the other causes of joint pain which can include non-specific arthralgia, osteonecrosis, lupus-like syndrome in relation to anti-TNF therapy, and corticosteroid withdrawal arthralgia. Azathioprine-related arthralgia usually occurs early in treatment and resolves rapidly on stopping the drug. The axial spondyloarthropathy has a much more disabling long-term course, and early diagnosis is vital, but can be very delayed. ${ }^{1106}$ Low back pain in young people (under 45 years), for more than 3 months that is improved with exercise and not relieved by rest, pain that is worse in the latter part of the night, and morning stiffness lasting more than $30 \mathrm{~min}$, should trigger investigation with MRI scanning (sagittal images of cervicothoracic and thoracolumbar regions with T1 and STIR images, and coronal/oblique sacroiliac joints with T1 and STIR) and referral to a rheumatologist, as plain radiology of the spine and sacroiliac joints will miss most early disease. HLA-B27 is less often positive in IBD-associated axial arthropathy. Specialist assessment and physiotherapy can prevent long-term disability, ${ }^{1107}$ and initial treatment is with NSAIDs, early progression to treatment with anti-TNF agents often being necessary, as sulfasalazine and methotrexate are not effective. ${ }^{1108} 1109$ Simple analgesia is preferable to NSAIDs, but NSAIDs are more effective.

Statement 120. We suggest that IBD patients with disabling fatigue in whom no correctable metabolic deficiency or active disease is found, or where fatigue persists despite addressing these factors, may be directed to non-pharmacological therapies. Patients may be interested in supportive psychotherapy, stress management or graded exercise (GRADE: weak recommendation, low-quality evidence. Agreement: $89.1 \%$ ).
Statement 121. We recommend that the mainstay of symptom relief for IBD-associated arthropathy which is related to IBD activity should be through control of intestinal inflammation, physiotherapy and simple analgesia (GRADE: strong recommendation, very low-quality evidence. Agreement: $100 \%$ ).

\subsubsection{Safety of NSAID use in IBD}

Data on non-selective NSAIDs is conflicting. A long-term study in over 600 IBD patients showed that low-dose NSAIDs were safe, but standard doses caused a rise in disease activity scores (particularly in Crohn's colitis), but not relapse. ${ }^{1110}$ Short-term use is safe, particularly using low doses where the IBD is well-controlled, with a relapse risk of about 20\%. ${ }^{11111112}$ A meta-analysis of seven studies of selective COX-2 inhibitors in IBD showed a risk of flare of $14 \%$ (95\% CI 6.7 to 24.4 ) with no difference to placebo (relative risk $0.86,95 \%$ CI 0.39 to 1.88$)$ ), ${ }^{1113}$ in keeping with a Cochrane analysis. ${ }^{1114}$ The risk of flare could be higher when given to patients with active IBD, as COX-2 is important in intestinal repair mechanisms.

\subsection{Other IBD therapy}

\subsubsection{Probiotics, prebiotics and synbiotics in IBD}

A systematic review assessed 21 studies in UC. ${ }^{1115}$ Nine were trials of induction therapy (seven using probiotics and one each prebiotic and synbiotics). The majority assessed the addition of the investigational treatment alongside standard therapy (mostly 5-ASA), with standard therapy alone being used in the control group. Study quality was variable, many being small trials, but overall the majority showed benefit, with statistical significance in 16 of the studies. The largest studies used a mixture containing eight probiotic organisms and overall there is evidence of benefit for this treatment in UC as an addition to standard therapy, with insufficient evidence to recommend other agents. A Cochrane review of probiotics in induction therapy published in 2007 (and so not including the largest studies reported in the systematic review) concluded that there was insufficient evidence to recommend probiotic therapy in induction of remission, but that it may improve symptoms in mild to moderate colitis. ${ }^{1116}$ The remaining trials evaluated maintenance therapy, some with remission induction followed by maintenance therapy. There was a modest benefit in a number of studies, and some showed equivalence to 5-ASA therapy. A variety of agents were used in these studies. Again, a Cochrane review in 2011 concluded that there is insufficient high quality evidence to show benefit in maintaining remission. ${ }^{1117}$ One study comparing a prebiotic, a probiotic and a synbiotic in UC using quality of life as an end-point concluded that synbiotic therapy was more effective than the other treatments. ${ }^{1118}$ In conclusion, probiotic therapy may have modest benefits in UC, but should not be routinely used. By contrast to $\mathrm{UC}$, there is no evidence of any benefit in the current literature for these treatments in Crohn's disease. ${ }^{1115}$

Statement 122. We recommend that for IBD-related arthropathy which is not related to IBD activity, rheumatology referral, physiotherapy and simple analgesia should be offered (GRADE: strong recommendation, very low-quality evidence. Agreement: 93.6\%). 
Statement 123. We suggest that short-term use of NSAIDs is safe if IBD is in remission, but long-term use or use in active disease carries more risk of worsening IBD symptoms. There is no high-quality evidence that COX-2 inhibitors are safer than non-selective agents (GRADE: weak recommendation, very low-quality evidence. Agreement: 81.4\%).

\subsubsection{Faecal microbial transplantation}

To date there have been four randomised placebo-controlled trials of faecal microbiota transplantation (FMT) for the treatment of UC, with three meeting their primary end-point showing a significant improvement in remission compared with placebo. ${ }^{1119-1122}$ An open-label study showed that benefit is more evident in UC than Crohn's disease. ${ }^{1123}$ The published trials vary greatly in route and frequency of FMT administration, ${ }^{1124}$ with evidence that improved microbial diversity can persist for 4-8 weeks, ${ }^{119} 1125$ but does not persist after 1 year, ${ }^{119}$ and growing evidence that colonic rather than nasoduodenal administration may be safer and more effective. ${ }^{1126}$ In spite of these encouraging data, FMT remains an investigational treatment for use only in clinical trials in IBD. ${ }^{1127}$

\subsubsection{Complementary and alternative therapy for IBD}

Complementary and alternative medicines (CAMs) include diverse medical and healthcare strategies which are considered to fall outside conventional treatment. Such interventions include herbal treatments, pre/probiotics, acupuncture and are often part of traditional Chinese medicine. There is evidence that $30-50 \%$ of patients with IBD use CAMs and this tends to be higher in women than men. ${ }^{1128} 1129$ While there is no evidence to recommend the routine use of CAMs, certain agents have shown early promising signals in pilot studies. Two promising candidates are curcumin, which is the active ingredient of turmeric (Curcuma longa) ${ }^{1130-1132}$ and fish oils, where there is good evidence of anti-inflammatory effects, but the largest and most rigorous trial (in Crohn's disease) showed no benefit. ${ }^{1133}$ No recommendations can be made due to lack of sufficient highquality evidence regarding efficacy and dose and larger studies are needed. Helminth therapy remains interesting despite the negative result of a randomised controlled trial in UC. ${ }^{1134}$ The results of a similar trial in Crohn's disease remain to be reported. Anecdotal evidence supports the use of acupuncture although there are no controlled trials and, in this case, blinding is an obvious problem. Moderate exercise has shown no harm in IBD and a positive benefit in terms of bone health. ${ }^{1135}$

Further data are required to move promising CAMs into the category of conventional treatment. ${ }^{1136}$ Despite the absence of robust evidence supporting the use of CAMs in IBD, there is evidence that many patients use CAMs. ${ }^{1128}$ It is good practice to enquire about the use of CAMs as patients are often either reluctant to mention this, given the negative social and legal connotations associated with cannabis for example, ${ }^{1137}$ or may not

Statement 124. We suggest that faecal microbiota transplantation (FMT) shows some evidence of benefit in ulcerative colitis and should be used in the context of clinical trials until further high-quality evidence clarifies the potential for benefit and optimal administration protocol (GRADE: weak recommendation, moderate-quality evidence. Agreement: $93.3 \%)$. realise that they are worth mentioning (eg, acupuncture, herbal supplements, exercise). It should be emphasised that they should continue conventional treatments of proven value even if they choose to use CAMs in addition.

\subsubsection{Cannabis extracts}

There is increasing interest in use of cannabidiol (CBD) in medicine. This does not have the psychotropic effects of the delta ${ }^{9}$-tetrahydrocannabionol (THC) component in cannabis. CBD can have beneficial effects in the gut including reduction in nausea, reduced gastric acid secretion, anti-inflammatory effects and reduction in intestinal motility. Cannabis use has been reported to improve IBD symptoms, ${ }^{1138}$ and many patients claim that it has beneficial effects. ${ }^{1139} \mathrm{~A}$ small double-blind randomised trial in 60 patients compared an oral CBD-rich botanical extract with placebo in mild-moderate active UC. At 10 weeks, the primary end-point of remission was achieved in $28 \%$ on CBD versus $26 \%$ on placebo (non-significant). The treatment caused side effects of dizziness and somnolence (likely due to the residual THC content of the botanical extract). There was some evidence of symptom improvement in the per protocol analysis. ${ }^{1140}$ A previous small randomised double-blind study in 21 Crohn's disease patients compared smoking two marijuana cigarettes (containing $115 \mathrm{mg}$ of THC) to a cannabis flower extract without THC over 8 weeks. The primary end-point of clinical remission was met in $45 \%$ on active treatment compared with $10 \%$ on placebo, but the difference was not significant. ${ }^{1141}$ Further adequately powered studies are needed, with rigorous blinding, and objective measures of inflammation as well as symptoms.

\subsection{Chemoprevention and colorectal cancer surveillance}

IBD is a widely accepted risk factor for the development of colorectal cancer (CRC). CRC accounts for one in six deaths in UC, ${ }^{1142}$ with median age younger than that of sporadic CRC. ${ }^{1143}$ There is a wealth of data from large national and international population-based studies demonstrating increased risk of CRC with UC, and CRC or small bowel neoplasia with Crohn's disease. ${ }^{1144-1146}$ A case-control study of 68 cases and 136 controls showed that the severity of inflammation, both endoscopic and histological, determines the risk of colorectal neoplasia in UC. ${ }^{1147}$ This has been supported by a cohort study demonstrating that histological inflammation predisposes to advanced neoplasia. ${ }^{1148}$ Case-control studies have shown that the presence of post-inflammatory polyps, as a marker of previous severe inflammation, increase the risk of CRC in UC. ${ }^{28} 11491150$ However, a recently published retrospective multicentre cohort study including 462 patients with post-inflammatory polyps confirmed their association with greater severity and extent of colonic inflammation and colectomy risk but did not observe an association with colorectal neoplasia. ${ }^{1151}$ Finally, the age of onset at diagnosis and the influence therefore of the length of disease in individuals (as stratified by phenotypic classification systems) must be considered as important factors for surveillance, given the association between length of disease duration and the development of cancer. ${ }^{1144} 1152$

\subsubsection{Surveillance ileocolonoscopy}

Long duration of disease increases the risk of developing IBD-associated CRC, with the risk starting to increase at about 10 years after diagnosis, by which time meta-analysis suggests the incidence is $2-3 \%$. ${ }^{1143} 1153$ Later studies have shown the cumulative incidence to be $1 \%$ at 10 years, $3 \%$ at 20 years and $7 \%$ at 30 years. ${ }^{1154}$ This change over time may reflect the increased 
implementation of surveillance strategies, the introduction of drugs that control inflammation more effectively, or the changing approach to maintenance therapy or colectomy. ${ }^{155}$ Current UK, European and USA endoscopy guidance suggests commencement of surveillance endoscopy for dysplasia in IBD should begin at between 6 and 10 years following diagnosis. ${ }^{1156-1158}$ A Cochrane review $^{1159}$ evaluated three UC case-control studies ${ }^{1160-1162}$ and concluded there was evidence for surveillance resulting in earlier detection stage and better prognosis, with indirect evidence of cost-effectiveness. A study of 149 IBD-associated cancers (59 Crohn's disease, 90 UC) showed that those with prior surveillance had greater 5-year survival and earlier tumour stage at detection. ${ }^{1163}$ A review of the St Mark's programme showed surveillance may reduce the risk of advanced and interval colorectal cancer incidence. ${ }^{1164}$ Those with extensive disease carry the highest risk of colorectal cancer, whereas left-sided colitis patients have an intermediate risk and those with proctitis only have no increased risk. ${ }^{1165}$ Extent therefore should determine the appropriate surveillance interval. Patients with primary sclerosing cholangitis (PSC) should have annual surveillance colonoscopy from diagnosis. ${ }^{1166}$ For all other patients, surveillance should begin 8 years after diagnosis, or after the onset of symptoms if there was a significant delay before diagnosis. Surveillance technique, including use of chromoendoscopy, is set out in the SCENIC consensus guidelines, ${ }^{1167}$ and the surveillance interval after the initial surveillance should be determined according to degree of inflammation, extent, presence of post-inflammatory polyps or strictures, dysplasia, PSC or family history of colorectal cancer under the age of 50 years. ${ }^{1155} 1156$ Patients with proctitis and no evidence of proximal progression do not require ongoing surveillance colonoscopy. A summary adapted from the 2010 BSG and 2015 SCENIC guidance is presented in box 11 .

\subsubsection{IBD-related cancer chemoprevention with mesalazine}

Although there is a lack of randomised prospective data regarding chemoprevention of CRC in IBD, a number of studies have shown a protective effect of 5-ASA on the risk of IBD-associated CRC. A systematic review suggests the drug has multiple biological effects that protect against neoplasia. ${ }^{1168}$ These include stabilisation of cell cycle regulation, scavenging of reactive oxygen or nitrogen species, inhibition of TNF $\alpha, N F-\kappa B$ and TGF $\beta$ pathways, interference with Wnt/beta-catenin signalling and antimicrobial activity. A systematic review and meta-analysis of eight studies contained 867 cases of colorectal neoplasia and 143 of non-cancer dysplasia. Prescription of 5-ASA is associated with OR of $0.6(95 \%$ CI 0.4 to $0.9, p=0.04)$ for development of CRC, ${ }^{1169}$ although the advantage was only seen in studies from referral centres, not population-based studies.

Two more recent meta-analyses have been published in 2017, confirming that 5-ASA reduces the risk of CRC in UC. ${ }^{1170} 1171$ Sulfasalazine was not protective. A dose-dependent protective effect was noted with 5-ASA with a minimum dose of $1.2 \mathrm{~g}^{1169}$ or 2 g. ${ }^{171}$ It remains unclear whether the protective effect of 5-ASA simply represents more effective mucosal healing rather than a specific drug effect. In patients who are also taking

Statement 125 . We recommend that IBD patients with colonic disease should be offered ileocolonoscopy 8 years after symptom onset to screen for neoplasia, to determine disease extent and decide on the frequency of ongoing surveillance (GRADE: strong recommendation, very low-quality evidence. Agreement: $89.1 \%$ ). immunosuppressive drug therapy there are no data to show that 5-ASA has additional benefits, whereas a recent retrospective study demonstrated that stopping 5-ASA in patients on anti-TNF therapy was not associated with a worse disease course. ${ }^{1172}$

\subsubsection{IBD-related cancer chemoprevention with thiopurines}

Inflammation may in part promote tumorigenesis, ${ }^{1173}$ so understanding the impact of immune-modifying therapy on cancer development is an important area of unmet clinical need. There are currently no randomised prospective data interrogating the effect of immunomodulatory agents (including thiopurines, methotrexate, calcineurin inhibitors, anti-TNF and anti-integrin agents) on the risk of IBD-associated CRC, and evidence is based on cohort studies. ${ }^{1165} 11741175 \mathrm{~A}$ recent meta-analysis from 2017 of 24 studies including 76999 patients concluded there was evidence of a protective role of thiopurines in highgrade dysplasia and CRC prevention in UC, but not Crohn's disease. ${ }^{1176}$ Potential benefit in reducing colorectal cancer risk must be weighed against the very small increase in risk of lymphoma, particularly in older patients.

There are no randomised prospective data on the effect of folic acid or antioxidants (vitamin A, C, E, selenium and beta-carotene) on the risk of CRC in IBD. Red blood cell folate is reduced in IBD patients who develop CRC compared with those who do not. ${ }^{1177}$ Two case-control studies have shown a non-significant dose-dependent reduction in CRC with folic acid supplementation. ${ }^{1178} 1179$

\subsection{Anti-TNF, vedolizumab, ustekinumab and tofacitinib therapy during pregnancy}

5.14.1 Fetal and infant exposure to anti-TNF drugs

Active transfer of $\mathrm{IgG}$ from the maternal to fetal circulation occurs at the surface of the syncytiotrophoblast placental layer through the selective binding of the $\mathrm{Fc}$ gamma portion of the maternal IgG antibody to the fetal circulation. ${ }^{1180}$ Active transport of IgG starts at approximately week 13 of gestation progressing continually until delivery with a preferential transport of IgG1 followed by IgG4, IgG3 and then IgG2..1180 1181 Infliximab, adalimumab and golimumab are IgG1 monoclonal antibodies, whereas certolizumab is a Fab fragment of IgG1 (without the Fc portion of IgG1) and so there is significantly less transfer through the placenta. Infliximab levels were detectable for up to 7 months and adalimumab levels remained detectable for up to 11 weeks from birth. ${ }^{1181}$ The use of infliximab and adalimumab during pregnancy has been associated with fetal and cord blood levels of drug up to fourfold higher than in maternal blood. ${ }^{1181-1183}$ A recent study showed that median cord blood drug concentrations were more than the median maternal drug concentrations at the time of birth for infliximab and adalimumab, with a much longer time for drug clearance in infants with the mean time for drug clearance of 7.3 months for infliximab (95\% CI 6.2 to 8.3 ) and 4 months (95\% CI 2.9 to 5.0$)$, respectively $(\mathrm{p}<0.0001) .{ }^{1184}$ In some infants, drugs remained detectable until 12 months of age.

Discontinuing anti-TNF therapy in the second trimester will limit drug exposure during the time of highest transmission of immunoglobulins from the mother to the fetus. Although the timing of the last anti-TNF administration appears to correlate with maternal serum and cord blood levels, this is not so in a linear fashion with variability due to differences in maternal dose and interval, individual pharmacokinetics and the immaturity of the newborn reticuloendothelial system. There is 
Box 11 Colonoscopy surveillance in patients with IBD to detect and manage dysplasia/neoplasia

Commencement of colonoscopy surveillance:

- 8 years after IBD symptom onset

- From diagnosis if primary sclerosing cholangitis

Next surveillance interval (summary of 2010 BSG update guidance) $*:^{1156}$

- Lower risk: next surveillance in 5 years if extensive colitis with no active endoscopic or histological inflammation or left sided colitis or Crohn's colitis affecting $<50 \%$ of colon

- Intermediate risk: next surveillance in 3 years if extensive colitis with mildly active endoscopic or histological inflammation or post-inflammatory polyps (often termed 'pseudopolyps') or family history of colorectal cancer in first degree relative aged $\geq 50$ years

- Higher risk: next surveillance in 1 year if extensive colitis with moderate/severely active endoscopic or histological inflammation or if stricture or dysplasia in last 5 years or primary sclerosing cholangitis (including post-orthotopic liver transplant) or family history of colorectal cancer in first degree relative aged $<50$ years

In determining next surveillance interval, consider patient preference, presence of multiple post-inflammatory polyps, age and comorbidity, accuracy and completeness of examination. If previous colectomy and ileostomy, or colectomy and ileorectal anastomosis: retained rectum should have surveillance examination. Retained rectum after surgery is more difficult to inspect and so proctectomy with/without pouch reconstruction should normally be considered.

Methods of detection*:

- Surveillance should be performed where possible during disease remission, though may not be possible in some patients, and chronic active disease is a risk factor for dysplasia.

Statements published as part of 2015 SCENIC international consensus: ${ }^{1167}$

- When performing surveillance with white-light colonoscopy, high definition is recommended rather than standard definition (strong recommendation, low-quality evidence)

- When performing surveillance with standard-definition colonoscopy, chromoendoscopy is recommended rather than white-light colonoscopy (strong recommendation, moderatequality evidence)

- When performing surveillance with high-definition colonoscopy, chromoendoscopy is suggested rather than white-light colonoscopy (conditional recommendation, lowquality evidence)

- When performing surveillance with standard-definition colonoscopy, narrow-band imaging is not suggested in place of white-light colonoscopy (conditional recommendation, low-quality evidence).

- When performing surveillance with high-definition colonoscopy, narrow-band imaging is not suggested in place of white-light colonoscopy (conditional recommendation, moderate-quality evidence).

- When performing surveillance with image-enhanced high-definition colonoscopy, narrow-band imaging is not suggested in place of chromoendoscopy (conditional recommendation, moderate-quality evidence).

\section{Box 11 Continued}

Colonoscopically targeted biopsies are recommended over random colonic biopsies. ${ }^{1289}$ If taking random biopsies, 2-4 biopsies should be taken every $10 \mathrm{~cm}$. ${ }^{1156}$

Management of dysplasia*:

Statements published as part of 2015 SCENIC international consensus: ${ }^{167}$

- After complete removal of endoscopically resectable polypoid dysplastic lesions, surveillance colonoscopy is recommended rather than colectomy (strong recommendation, very-low quality evidence).

- After complete removal of endoscopically resectable non-polypoid dysplastic lesions, surveillance colonoscopy is suggested rather than colectomy (conditional recommendation, very-low quality evidence).

- For patients with endoscopically invisible dysplasia (confirmed by a Gl pathologist), referral is suggested to an endoscopist with expertise in IBD surveillance using chromoendoscopy with high-definition colonoscopy (conditional recommendation, very low-quality evidence). If dysplastic lesions cannot be resected completely due to extent or multiplicity, referral to MDT for discussion regarding definitive management including surgical options should be arranged.

\begin{abstract}
${ }^{*}$ Neither extensive systematic review nor consensus voting has been undertaken as part of the current guideline development process regarding subsequent surveillance intervals after initial surveillance colonoscopy, or methods to detect dysplasia. Instead a summary is adapted/reproduced from the 2010 BSG $^{1156}$ and 2015 SCENIC ${ }^{1167}$ guidance.
\end{abstract}

more variability for infliximab levels than for adalimumab in maternal blood during pregnancy. ${ }^{1185}$ This may suggest a role for therapeutic drug monitoring while using infliximab in pregnancy.

While low levels of infliximab, adalimumab, certolizumab, natalizumab and ustekinumab can be detected in breast milk from mothers receiving these biologics, breastfed infants of mothers receiving biologics, immunosuppressants or combination therapy have similar risks of infection and similar milestone achievement at 12 months to non-breastfed infants or infants unexposed to these drugs. ${ }^{1186}$

\subsubsection{Cessation of anti-TNF therapy in the second trimester in} quiescent IBD

Active IBD prior to conception is associated with poor pregnancy outcomes, including premature delivery, low birth weight and higher spontaneous abortion, compared with quiescent or controlled disease. ${ }^{1187-1190}$ Discontinuing anti-TNF therapy during pregnancy, in order to minimise exposure to the fetus, is feasible if there is no significant increase in the risk of disease flare. Case-control and cohort studies of pregnancy in women

Statement 126. We recommend that patients with ulcerative colitis or IBD-U with left-sided or more extensive disease should be advised to take mesalazine in doses of at least $2 \mathrm{~g}$ daily to reduce risk of colorectal cancer (GRADE: strong recommendation, moderate-quality evidence. Agreement: $95.5 \%$ ). 
Statement 127 . We suggest that thiopurines may reduce risk of colorectal cancer in patients with ulcerative colitis and IBD-U, but evidence for a chemopreventative role from methotrexate, calcineurin inhibitors, anti-TNF and anti-integrin agents is lacking at present and they cannot be currently recommended solely for chemoprevention against colorectal cancer (GRADE: weak recommendation, low-quality evidence. Agreement: 93\%).

with quiescent IBD do not show any increase in risk of flare if anti-TNF therapy is stopped at week 25-30. ${ }^{1182} 1191$ Anti-TNF medications therefore may be safely discontinued in the second trimester in women with quiescent disease.

\subsubsection{Continuation throughout pregnancy in IBD patients at high risk of flare}

A Danish study of 219 women with IBD treated in the third trimester with anti-TNF therapy revealed no increased risk of low birth weight or preterm birth associated with the drug. ${ }^{1192}$ The same group found that, in women with moderate to severe IBD on anti-TNF during pregnancy, 66\% experienced disease activity during their pregnancy. Disease activity was associated with low birth weight (OR 2.05) and preterm birth (OR 2.64, increasing to an OR of 3.6 in moderate to severe disease). ${ }^{1192}$ There is also evidence that discontinuation of therapy may be associated with a risk of relapse during pregnancy and in the postpartum period. ${ }^{181-1184}$ Additionally, low trough levels may lead to anti-drug antibody formation with subsequent loss of response to treatment.

\subsubsection{Risk to the fetus of continuing anti-TNF until delivery}

A recent UK study of 364363 singleton pregnancies including 1969 in women with IBD revealed only a small increased risk of preterm birth, and in Crohn's disease a higher risk of low birth weight or post-partum haemorrhage. ${ }^{193}$ Most women with IBD will have an uncomplicated pregnancy. Despite significant fetal exposure to anti-TNF drugs, there is no evidence that continuing anti-TNF therapy through pregnancy has a negative impact on the pregnancy or neonatal outcomes, including neonatal susceptibility to infection. Meta-analysis of six studies confirmed no increased risk of adverse pregnancy outcomes, congenital abnormality, preterm birth or low birth weight. ${ }^{1194} \mathrm{~A}$ study of all 1097 individual safety reports received by the Food and Drug Administration adverse event reporting system did not show an increased risk of maternal or neonatal adverse outcome for thiopurine, anti-TNF or combined therapy. ${ }^{1195}$ In contrast, a prospective study of 80 pregnancies in women exposed to anti-TNF showed no adverse neonatal outcome on anti-TNF monotherapy but did report an increased risk of neonatal infection (bacterial infections in 5\% and viral infections in 20\%, all with benign course) with combined therapy with thiopurines (relative risk $2.7,95 \%$ CI 1.09 to $6.78, \mathrm{p}=0.02$ ). ${ }^{1184}$ The largest

Statement 128. IBD patients receiving anti-TNF therapy should be counselled about the risks and benefits of continuing treatment throughout pregnancy. We suggest that, for patients with active disease or a high risk of relapse, it may be advisable to continue the drug throughout, while for those with inactive disease who wish to discontinue therapy it may be reasonable to stop at the start of the third trimester (GRADE: weak recommendation, very low-quality evidence. Agreement: 97.7\%). retrospective cohort study to date has recently published the outcomes in 1457 pregnancies in women exposed to anti-TNF therapy for IBD. ${ }^{1196}$ Treatment was associated with a higher risk of overall maternal complications (adjusted OR 1.49, 95\% CI 1.31 to 1.67 ) and infections (adjusted OR 1.31 , 95\% CI 1.16 to 1.47). Ongoing use of anti-TNF therapy beyond 24 weeks did not increase maternal complications, but interrupting treatment was associated with relapse (adjusted OR 1.98, 95\% CI 1.25 to 3.15). No increased infection rates were seen in children born to mothers exposed to anti-TNF in this study (adjusted OR 0.89, $95 \%$ CI 0.76 to 1.05$)$.

\subsubsection{Long-term outcomes of anti-TNF therapy during pregnancy}

A multicentre retrospective study from the Netherlands has recently been presented in abstract form reviewing outcomes up to 5 years for children born to mothers with IBD. ${ }^{1197}$ Of 1000 children born, $20 \%$ had been exposed to anti-TNF in utero, $24 \%$ to thiopurine monotherapy and $<1 \%$ to systemic steroids. Neither anti-TNF nor thiopurine exposure was associated with adverse birth outcomes, whereas use of steroids was associated with preterm birth (adjusted OR 2.67, 95\% CI 1.59 to 4.47). Exposure to IBD therapy did not influence long-term adverse health outcomes, adverse reaction to vaccination or infection rates.

The TEDDY study, a retrospective multicentre study, followed children born to women with IBD who were exposed to anti-TNF either during pregnancy or within 3 months prior to conception and a non-exposed comparator group. ${ }^{1198}$ The incidence rate of severe infections, however, was similar between groups and anti-TNF exposure during pregnancy was not associated with a higher risk of severe infections (HR 1.2, 95\% CI 0.8 to 1.8 ). The exposed group had more Caesarean sections, more with low birth weight and more intensive care unit admissions.

The PIANO registry (Pregnancy and Neonatal Outcomes in Women with IBD), a prospective USA registry with over 1000 patients, has presented data on up to 4 years of follow-up in patients exposed to biological therapy during pregnancy. The use of anti-TNF during pregnancy was not associated with developmental delay or increased risk of infection in this cohort. $^{1199}$

Although the majority of data are for infliximab and adalimumab, there are data to suggest that golimumab is also associated with a low risk of adverse maternal and neonatal outcome. $^{1200}$

In conclusion, a decision to stop anti-TNF therapy between weeks 22 and 24 of gestation must hinge on disease quiescence (sustained remission for 12 months prior to conception with endoscopic and radiological evidence), driven by strong patient preference. Patients at increased risk of flare may continue anti-TNF therapy throughout pregnancy.

\subsubsection{Infant vaccinations after exposure to biologics}

Offspring exposed to anti-TNF drugs, vedolizumab and ustekinumab in utero are able to mount appropriate antibody responses to inactivated vaccines and should complete the inactivated vaccine programme as scheduled. ${ }^{1201}$ A multicentre observational retrospective study from 22 French gastroenterology departments was recently presented in abstract form. ${ }^{1202}$ The study aimed to determine the rate and tolerance of live vaccines in children before and following 6 months of life when exposed in utero to anti-TNF therapy and in children breastfed by mothers receiving anti-TNF. Anti-TNF therapy was stopped 
Statement 129. We suggest that BCG vaccination (if indicated) should be withheld until at least 6 months after birth, and rotavirus vaccine should not be given, for infants exposed in utero to biological therapies. Non-live vaccinations may be given according to standard vaccination schedules (GRADE: weak recommendation, very low-quality evidence. Agreement: 97.7\%).

before 26 weeks gestation in $74(52 \%)$ women and resumed following delivery in 92\%. Sixty-three women (45\%) breastfed and no complications were reported. BCG vaccination was administered in 33 children $(27.5 \%)$ prior to 6 months in 19 children (16\%). One local abscess was reported with 'favourable evolution'. Seven children received rotavirus, five children before 6 months. One case of fever was reported. MMR was administered in 72 children (60\%), before 6 months in six cases.

Data from the Dutch National Vaccination Programme in children exposed in utero to anti-TNF therapy shows that child vaccination against hepatitis $\mathrm{B}$ is effective compared with a control group. ${ }^{1203}$ There were no differences in birth outcomes, growth and infections in the first year of life.

Thus, non-live vaccination should be given as usual in infants exposed in utero to anti-TNF drugs, BCG should be deferred to 6 months of age and rotavirus should not be given at all (as there is no value in giving rotavirus later than 6 months).

\subsubsection{Vedolizumab, ustekinumab and tofactinib in pregnancy}

There are very limited data about outcomes of pregnancies with vedolizumab. A review of pregnancies that occurred within the vedolizumab trials revealed 27 unintended pregnancies in female participants and 19 pregnancies in female partners of male participants. ${ }^{1204}$ No significant safety concerns were identified, although most women did not continue vedolizumab through to term. One case series presented four pregnancies while on vedolizumab, all successful (live offspring, not premature, normal APGAR scores, achieved developmental milestones). ${ }^{1205}$ The data for ustekinumab in pregnancy are also limited to case studies and registry data. These data from rheumatology suggest that ustekinumab is not associated with increased risk of miscarriage or congenital malformation. ${ }^{1206}$ However, controlled data are lacking. Data in pregnancy in women with IBD pregnancy are limited to case reports. ${ }^{1207}$ Data are also limited for tofacitinib in pregnancy associated with UC, where 11 cases of maternal exposure and 14 cases of paternal exposure have been identified across intervention studies. ${ }^{1208}$ Outcomes include 15 healthy infants, with no neonatal or fetal deaths, no congenital malformations, two spontaneous abortions and two medical terminations.

The lack of data confirming safety and the consequences of withdrawing treatment should be discussed as part of pre-conception counselling. It is unknown whether exposed infants are susceptible to pathogenic infection from live vaccines. Until further data are available, it is recommended that the same practice that is used for anti-TNF drugs is applied to the newer biological agents. General guidance on pregnancy in IBD patients, summarised from ECCO and Toronto guidance, ${ }^{1209} 1210$ is shown in box 12 .

\subsection{Genetics}

Most children and the vast majority of adults with IBD do not have a monogenic predisposition to intestinal inflammation. However, this possibility should be considered in individuals either with very early onset of disease (ie, often in infancy, but up to 5 years old by the definition) or early onset in whom there are atypical features including susceptibility to unusual or frequent infections (suggesting a possible underlying immune deficiency). Mutations in the IL-10 signalling pathway have a Mendelian inheritance pattern and complete penetrance of the IBD-like phenotype, while a number of other genetic defects that disturb intestinal epithelial barrier function or affect innate and adaptive immune function have incomplete penetrance of intestinal inflammation. The importance of not missing such cases lies in the aggressive nature of the IBD-like phenotype and the fact that they are often very resistant to conventional IBD therapies. Some, such as XIAP and IL-10 receptor mutations, require allogeneic bone marrow transplantation for effective treatment and to prevent premature death. Increasingly, the diagnostic pathway relies on exome sequencing, but the analysis is complex and accurate diagnosis of the multiplicity of monogenic conditions that can produce IBD-like phenotypes in or soon after infancy relies on the laboratory being familiar with this clinical spectrum. ${ }^{1211}$

Pharmacogenetic testing for thiopurines is discussed in the Section on 5.2.2.1: Common Disease Considerations, Thiopurine methyltransferase and NUDT15.

\subsection{Travel advice for IBD patients}

Patients can be very anxious about the risks of travelling abroad and issues surrounding vaccination during treatment for their IBD. Knowledge among patients and health professionals is poor, so education for both is important. ${ }^{1212} 1213$ The IBD Passport website provides comprehensive information about travel with IBD (www.ibdpassport.com). Comprehensive information, aimed at health professionals but available to the public, is also available in the CDC Travellers Health - Yellow Book (https:// wwwnc.cdc.gov/travel/page/yellowbook-home). Live vaccinations should not be given to patients on immunosuppressive drugs and can only be given a minimum of 3 months after stopping this treatment. In relation to travel, this will include yellow fever and oral cholera vaccine. Clear advice is provided in the UK Green Book. ${ }^{730}$

Patients should also be counselled about dietary precautions when travelling, managing their medication, dealing with diarrhoea and travel insurance. They should be advised not to stop immunosuppressive therapy during an exacerbation of symptoms or potential infection. ${ }^{1214}$ In fact, immunosuppressed IBD patients who may be travelling abroad need to be optimised medically to try and prevent opportunistic infection where possible. $^{1215}$

Travellers with IBD to developing countries (particularly if likely to be staying in accommodation with poor sanitation) are advised to carry a fluoroquinolone course (such as ciprofloxacin $500 \mathrm{mg}$ twice daily) for empirical self-treatment of traveller's diarrhoea. Azithromycin is comparable to quinolones for self-treatment of traveller's diarrhoea in certain circumstances: (i) patients who take a fluoroquinolone as part of their treatment for IBD; (ii) travellers to countries where endemic bacteria are known to have high levels of fluoroquinolone resistance; (iii) patients who have no response to a quinolone within 36-48 hours; (iv) pregnant women and children $<16$ years (for whom a fluoroquinolone is contraindicated). ${ }^{1216}$ Currently, there is no evidence to support empirical treatment with rifaximin. Overall, the immunocompromised traveller with diarrhoea should have a lower threshold than immune competent travellers for initiating self-therapy, but stool samples should be obtained 
Box 12 General guidance on pregnancy in IBD patients

Prior to conception:

- Education: Importance of keeping well ('you need to be well for your baby to be well'). Discuss potential adverse fetal outcomes of uncontrolled IBD (eg, pre-term birth and low birth weight), the risk of flare versus risk of current medications.

- Optimise: Is the patient as well as possible with their IBD? Investigate and, if necessary, treat.

- General health: Consider folic acid, nutrition, cervical smear, smoking cessation, vaccinations.

- Consider: Is the patient receiving the safest possible combination of medicines for pregnancy?

During pregnancy:

- Treat both maintenance and flares as normal with 5-ASA, thiopurines, anti-TNF, nutrition and steroids. Indications for surgery in pregnant women with IBD are the same for nonpregnant patients

- Use therapies with the best evidence base for safety in pregnancy.

- Use imaging as needed but minimise radiation exposure with emphasis where possible on ultrasound and MR. Essential endoscopic investigations only when needed for clinical decision making (ideally in second trimester).

- VTE prophylaxis is particularly important if in hospital (including following Caesarean section) or unwell.

- Involve the MDT and patient in your decisions and include an obstetrician with appropriate experience.

Delivery and postpartum:

- Mode of delivery should be determined by obstetric considerations and patient preference except active perianal disease and ileoanal pouch or ileorectal anastomosis where caesarean section is often preferred.

- VTE prophylaxis is important after Caesarean section.

- Medicines safe in pregnancy are also safe in breastfeeding and should continue.

- Breastfeeding is the preferred method of feeding and does not impact the course of IBD.

5-ASA, 5-aminosalicylic acid; IBD, inflammatory bowel disease; MDT, multidisciplinary team; TNF, tumour necrosis factor; UC, ulcerative colitis; VTE, venous thromboembolism.

Adapted from Van der Woude et al, ${ }^{1209}$ and Nguyen et al. ${ }^{1210}$

for microbiology in patients whose diarrhoeal symptoms do not respond to initial antibiotics. ${ }^{700}$ All patients should be advised to seek medical advice if they do not respond to simple measures. Returning travellers with diarrhoea should have stool tests for infection and a full blood count for eosinophilia. Travel and opportunistic infection are covered in more detail in recent ECCO guidance. ${ }^{700}$

Statement 130 . We suggest that genetic testing for monogenic disorders should be considered in adolescents and young adults who have had early onset (before 5 years of age) or particularly aggressive, refractory or unusual IBD presentations (GRADE: weak recommendation, very low-quality evidence. Agreement: $95 \%)$.
Statement 131. We recommend that IBD patients travelling abroad should receive pre-travel health advice, taking into account any immunosuppressive medication. Standard vaccination advice should be given, but those on immunosuppressive drugs should not receive live vaccination unless treatment has been discontinued for a minimum of 3 months (GRADE: strong recommendation, very low-quality evidence. Agreement: 95.7\%).

\section{SERVICE DELIVERY}

\subsection{IBD service and the multidisciplinary team}

A multidisciplinary team (MDT) approach to patient management is recommended by NICE Quality Standards and by expert opinion. ${ }^{1217-1219}$ MDT management of IBD helps to provide optimised and personalised care, based on available professional expertise, infrastructure and funding. ${ }^{1220-1224}$ Robust evidence in defining adult IBD MDT membership is lacking; however, best practice identifies a minimum per 250000 population of two gastroenterologists, two colorectal surgeons, 2.5 IBD nurses, 1.5 stoma nurses, 0.5 dietitian (allocated to Gastroenterology), 0.5 administrative support, one histopathologist, one radiologist and one pharmacist - all with arrangements for cover in the event of absence. ${ }^{1225}$ The wider MDT (see figure 7) should include a psychologist, paediatric IBD team, obstetrician, rheumatologist and dermatologist. ${ }^{1224}$ Input may be required from hepatology, oral medicine, ophthalmology, general practitioner, social workers and the nutrition support team.

The addition of an MDT coordinator has been shown to be effective in cancer management ${ }^{1226}$ and should be considered in IBD. However, patient advocacy has been shown to be lacking in some MDT meeting models. The systematic review of cancer MDTs demonstrated a biomedical approach to most decisions with nursing staff often not having an active role in decision-making and patient preferences frequently not considered as part of the process. ${ }^{1227}$ The IBD nurse is well placed to fulfil this role in the MDT. The patient should be provided with information and support at all stages of their illness to enable shared decision-making with the IBD specialist team. ${ }^{1225}$ In IBD cross-specialty discussion, decision-making and follow-up is of clear importance, especially in surgical management, ${ }^{1221} 1228$ and should be clearly documented.

\subsubsection{MDT meetings}

Timing of MDT meetings depends on the size of unit and should happen weekly, or frequently enough to ensure decision-making is not delayed, and should have enough capacity to deal with the number of patients. Acute or emergency care decisions should not be delayed for timetabled meetings but should happen separately between relevant specialists. In a UK nationwide audit, the presence of an IBD MDT was an independent factor associated with lower rates of steroid overuse. ${ }^{448}$

Good Practice Recommendation 26. IBD patients travelling abroad should be advised to take adequate supplies of medication with them and should not stop their immunosuppressive therapy. Those on immunosuppressive drugs should be advised to have a low threshold for treating possible traveller's diarrhoea with quinolones or azithromycin (Agreement: 95.7\%). 
Good Practice Recommendation 27. The IBD multidisciplinary team (MDT) should include a core membership consisting of gastroenterologist, colorectal surgeon, IBD specialist nurse, radiologist, dietitian, histopathologist and pharmacist, all of whom should have expertise in IBD. The MDT should have a designated coordinator and be able to access advice from the following disciplines where necessary for decisions about specific patients: stoma nurse, paediatric gastroenterologist, hepatologist, nutrition support team, psychologist, primary care physician, ophthalmologist, rheumatologist, dermatologist, obstetrician and social worker (Agreement: 97.9\%).

Limited evidence exists on the specific format of an MDT meeting; however, a colorectal MDT survey highlighted the need for a coordinator and the benefits of training in data management. ${ }^{1226}$ The cost-effectiveness of the MDT in secondary care has not been demonstrated mainly due to heterogeneity in study methodology. ${ }^{1229}$ However, a recent Delphi consensus of IBD service quality indicators emphasised the importance of team working and this can be delivered effectively through an MDT process with administrative support, either by a secretary or clerical officer. ${ }^{1230}$

\subsection{Quality improvement and audit}

There is much that can be done to improve the quality of care for IBD patients, both in adhering to evidence-based standards of care and also in improving the quality of patients' experience of their healthcare by provision of information, shared decision-making and improving access to their IBD team. ${ }^{1222}$ A recent Delphi consensus identified a core set of 56 quality indicators (12 structure, 20 process and 24 outcome). ${ }^{1230}$ Structure and process quality indicators highlight the need for

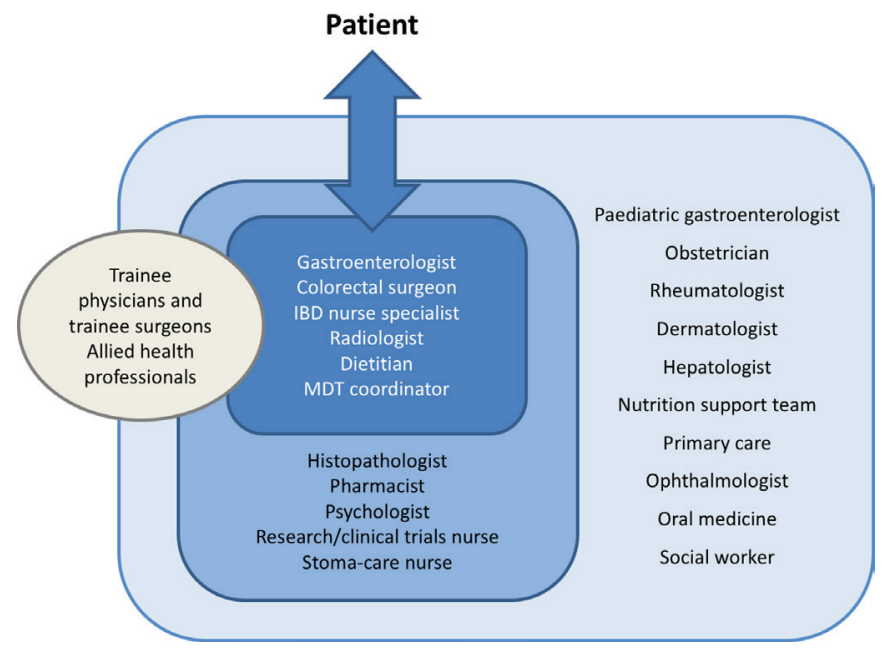

Figure 7 The IBD multidisciplinary team (MDT). It is important that the MDT meets regularly to review cases and make recommendations. All members of the team should have input as appropriate, but those in the inner box should be present at all regular MDT meetings. Staff in the middle box can and should be encouraged to attend these meetings, but this may not always be possible and they should nevertheless be encouraged to contribute reports or opinions. Staff in the outer box are part of the wider MDT and may contribute to team function through clinical input outside of these team meetings, such as through combined clinics or working to shared protocols. Staff in the grey circle should attend as a valuable part of their training.
Statement 132 . We recommend that regular multidisciplinary team (MDT) meetings should be held to discuss IBD patients with complex needs (GRADE: strong recommendation, very low-quality evidence. Agreement: 97.9\%).

multidisciplinary management and continuity of care, and that IBD units should be able to provide both outpatient and inpatient care. Outcome quality indicators focused on the adequate prophylaxis of disease complications and drug adverse events, the need to monitor appropriateness of treatment and the need to reinforce patient autonomy by providing adequate information and facilitating patients' participation in their own care. The IBD MDT is essential to this process. ${ }^{1218}$

\subsubsection{Rapid assessment}

All patients referred with suspected IBD should be seen by a specialist within 4 weeks of referral, but patients with severe symptoms may need urgent specialist assessment within a few days. ${ }^{1217}$ Patients experiencing a relapse of their IBD should have access to a member of the IBD team within five working days, and patients requiring radiological or endoscopic investigations should have tests performed within 4 weeks, or within 24 hours in an acute or emergency situation. ${ }^{1225}$

\subsubsection{Adverse event monitoring}

The UK National IBD Audit was a powerful tool to measure and enable IBD services to compare practice. Participation in the Audit was a catalyst for service improvement. ${ }^{1231}$ Monitoring and reporting adverse events are important aspects of service improvement. 12321233

\subsection{Electronic patient data collection for clinical management and audit}

The use of an IBD-specific patient management system is essential to capture clinical data on patients receiving immunosuppressive and biological drug therapy. It enables input and presentation of key information at point of care, facilitates effective drug monitoring, and collects data that can be used for audit and research. ${ }^{1234} 1235$ With the increasing availability of new drugs, including biosimilars, these data can be used locally, but also on a national basis to monitor safety and efficacy.

\subsection{IBD specialist nurses}

The role of the IBD specialist nurse is integral to the provision of a multidisciplinary service for IBD patients. ${ }^{1230} 1236$ Although there is little robust RCT evidence to demonstrate the cost-effectiveness of the role, ${ }^{1237}$ some suggest that the IBD nurse specialist role results in significant reduction in hospital admissions, emergency unit attendances and clinic visits as a result of contact with the nurse. ${ }^{1238} 1239$ Other small controlled studies suggest cost-effectiveness, ${ }^{1240}$ while some observational studies have suggested an improvement in patient care. ${ }^{1241-1243}$

IBD specialist nurses are often responsible for the management of medication and provision of disease information for patients. ${ }^{122012391244}$ Adherence to medication can be improved

Good Practice Recommendation 29. IBD units should have quality improvement and audit processes embedded within regular clinical activity (Agreement: 100\%). 
Good Practice Recommendation 28. For each IBD patient discussed in the MDT meeting, a formal record should be kept of management decisions reached (Agreement: 100\%).

by multiple different interventions including patient education, behavioural change, dose simplification, cognitive behavioural therapy and use of telemedicine. ${ }^{1245}$ A study of nurse telephone counselling in 524 patients with UC using cognitive-behavioural and motivational interview techniques to address cognitive and emotional barriers to adherence showed significant improvements in adherence, although there was no control group. ${ }^{1246}$ A Norwegian randomised study of nurse follow-up compared with gastroenterologist follow-up showed that outcomes were as good over 1 year in the nurse follow-up clinics as they were in the gastroenterologist clinics. ${ }^{1236}$ Time between symptoms of relapse and starting treatment was shorter in the nurse-led clinic and may relate to improved patient access to the IBD service.

\subsection{Telephone advice lines/telephone and virtual clinics}

IBD advice telephone or email services are an important communications pathway for IBD patients who need access to specialist support and advice, while not necessarily needing a clinic appointment or hospital admission. A Spanish study of $>1200$ calls showed that a specialist nurse could manage two-thirds, with the remainder requiring a doctor's input. ${ }^{1247}$ Nearly $90 \%$ were resolved by telephone call alone. $27 \%$ concerned IBD drug treatment, $25 \%$ related to disease concerns (mostly possible relapse), $25 \%$ related to administrative issues (such as appointment bookings) and $22 \%$ were queries regarding disease management. In an Irish survey, over one-third of calls related to flares or patient concerns, but $44 \%$ related to administrative issues, including blood test requests/results and repeat prescriptions. ${ }^{1242}$ Having a system to divert administrative queries to a secretary or clerical staff would free up time for IBD specialist nurses for more appropriate use of their skills-for example, carrying out vigilance and rescue work.

Following introduction of a telephone advice line in 2013 in a Scottish hospital, avoidance of consultant clinic and general practitioner appointments were estimated to have resulted in highly significant cost savings of over $£ 42000$ in a 5 -month period. ${ }^{1248}$ A study in Spain also suggested significant cost savings from availability of telephone consultations with an IBD nurse. ${ }^{1249}$ In Canada, a survey of over 1000 patients showed that many felt it difficult contacting their physicians, and 77\% stated they would make telephone contact with an IBD nurse if available in future, when having acute symptoms. The authors speculated that this would result in fewer emergency department attendances. ${ }^{1250} \mathrm{In}$ a USA study a survey was undertaken of telephone calls to nurse coordinators supporting IBD providers in a tertiary centre. ${ }^{1251}$ Patients with high call rates ( $>10$ per year) had more complex disease and drug treatments, markers of increased disease activity and high service utilisation. In order to effectively run telephone and email services, adequate funding and administrative support must be available.

Good Practice Recommendation 30. IBD services should collect data on adverse events and near misses. The service should participate in local and national audit and engage in ongoing quality improvement (Agreement: 100\%).
Good Practice Recommendation 31. Patient data on treatment with biological therapies and immunomodulators should be recorded to provide information for clinical management and to monitor adverse events and clinical outcomes for both local and national audit. Biological treatments should be recorded by brand name where biosimilar drugs are also available (Agreement: 97.8\%).

\subsection{Alternatives to clinic attendance for follow-up}

A recent USA study showed that patients attending outpatient clinic appointments travel more than 25 miles each way to attend, have to take half a day off work and incur personal out-of-pocket expense of 62 US dollars to attend. ${ }^{1252}$ Coupled with increasing demands on health service appointments, many strategies have been introduced to reduce the need for faceto-face clinic appointments for patients in stable long-term remission. This includes telephone clinics, email contact and other electronic management or web-based systems. Telephone contact as an alternative to clinics are acceptable to most patients, ${ }^{1253}$ with a Danish survey showing that $87 \%$ approved, particularly in younger patients who were working. ${ }^{1254}$ Quality outcome measures have been shown as comparable with telemedicine clinic in comparison to standard in-office appointment. ${ }^{1252}$ A pharmacist-led telephone clinic for patients on immunomodulators has been shown to be popular and effective. $^{1255}$

Supported self-management systems, including telephone contact, may improve monitoring and colonoscopic surveillance adherence for stable patients who could otherwise be lost to follow-up. ${ }^{1256}$ Other e-health technologies have gained popularity in recent years including smart phone applications and web-based interventions. These tools may serve as a method of engaging rural or isolated populations. ${ }^{1257}$ A review of six randomised controlled trials of a variety of distance management systems (web-based self-management and education, email contact, open-access clinics, and three that included telephone contact) showed a significant reduction in clinic visits with an average reduction from two to three down to one clinic visit per year. ${ }^{1258}$ The review suggested that more interaction between patient and professionals may be associated with greater benefits in terms of quality of life, but overall there was no significant change in quality of life, relapse rates or hospital admissions. A further recent systematic review identified six randomised controlled trials and nine observational studies showing improvements in relapse duration (18 days vs 77 days, $\mathrm{p}<0.001$ in one study), disease activity, improved medication adherence, quality of life, IBD knowledge, costs, reduced outpatient clinic attendance and high patient satisfaction with these technologies. ${ }^{1259}$ IBD services should have provision for remote follow-up methods, which should be offered to motivated patients who are happy not to attend conventional clinic follow-up appointments.

Good Practice Recommendation 32. Clinical nurse specialists are a vital part of the IBD team, where their role may include provision of cost-effective patient education, disease management and therapy monitoring, patient support, continuity of care, audit and rapid access for advice and review during disease flares (Agreement: 100\%). 
Statement 133. We recommend that IBD patients and primary care physicians should be provided with a dedicated IBD telephone line or email service to allow timely advice and care, particularly during times of relapse or to discuss managing medications or treatment side effects. Services must have adequate administrative and funding models (GRADE: strong recommendation, low-quality evidence. Agreement: 100\%).

\subsection{Self-management}

Guided self-management describes empowerment of patients to manage their condition and be involved in treatment decisions through provision of written information in understandable terminology regarding anticipated treatment or surveillance algorithms or when and how to contact primary or secondary care professionals. A randomised trial of guided self-management showed a reduction in clinic visits and more rapid treatment of flares in the self-management group. ${ }^{1260}$ There was no significant increase in quality of life overall. A subsequent randomised controlled trial recruiting 700 patients demonstrated that, over 1 year, self-management techniques led to fewer hospital visits (difference $-1.04 ; 95 \%$ CI -1.43 to -0.65 , $\mathrm{p}<0.001) .{ }^{1261}$ This was not associated with increased numbers of visits to primary care. Satisfaction and quality of life were similar between guided self-management and standard care. The self-management group reported increased confidence in being able to manage their condition. Longer term, specialist nurse contact was required in additional to guided self-management in order to ensure colorectal cancer surveillance is undertaken, that patients receive osteoporosis screening and that annual reviews are completed. ${ }^{1262}$

A systematic review, meta-analysis and meta-regression of 15 studies of self-management interventions showed that these strategies do result in some improvement in health-related quality of life, but there were no significant differences between the different components of the self-management strategy, perhaps because of the range of interventions that were included. ${ }^{1263}$ There was a trend for those with tailored management (eg, including guidance according to individual symptom diaries, personalised guided self-management advice, or individual psychotherapy support), for those with information components that improved IBD knowledge, and for those with detailed symptom management, to result in better outcomes. The only significant difference was shown between the two web-based programmes, which had a greater effect size compared with the remaining 13 with face-to-face interventions.

A multicentre controlled trial of telemedicine incorporating guided self-management has recently been published by a group in the Netherlands. The 'myIBDcoach' system utilises algorithms to request patients to complete questionnaires monitoring disease

Statement 134. We recommend that telephone or video virtual clinics can allow for the timely follow-up of stable IBD patients as an alternative to face-to-face clinic visits. These clinics need adequate administrative support and documentation, and methods to ensure contact with patients is maintained. Patients must have easy access to telephone or email advice and clinic access in the event of disease flare or patient concern about their IBD (GRADE: strong recommendation, low-quality evidence. Agreement: 95.7\%).
Statement 135. We suggest that IBD patients who wish to participate in guided self-management should be provided with education sessions and written or electronic personalised information about their management, with ongoing support and access to the IBD service in the event of relapse (GRADE: weak recommendation, moderate-quality evidence. Agreement: $93.6 \%)$.

activity between 3 months when in remission and weekly during a flare. Questionnaires are undertaken in preparation for outpatient review. Parameters above a pre-defined threshold were flagged to the IBD clinic. The system also includes a personalised output including visualised health parameters, PROs and quality metrics, and interactive patient-tailored information on topics including medications, adherence, smoking cessation, nutrition, self-management to prevent or reduce symptoms, fatigue, work productivity, anxiety and depression. It also contains a secure messaging service between patient and clinician. After 1 year, those assigned to telemedicine $(n=465)$ compared with standard care $(n=444)$ attended fewer outpatient clinics $(1.55$ vs $2.34, \mathrm{p}<0.0001)$ and had fewer hospital admissions $(0.05$ vs $0.10, \mathrm{p}=0.046)$. Quality of care scores did not vary between groups. $^{1264}$

\subsection{Primary care}

Primary care is often the first point of contact for IBD patients with deteriorating symptoms. Clear and comprehensive communication between hospital and primary care is essential (box 13). Recommended information should be included in correspondence between secondary and primary care. The development of local pathways taking into consideration available resources is encouraged. Patients with clinical features of severe disease, defined as six or more bloody stools per day plus one of temperature $>37.8^{\circ} \mathrm{C}$, heart rate $>90$ beats $/ \mathrm{min}$, anaemia $<105 \mathrm{~g} / \mathrm{L}$ or ESR $>30 \mathrm{~mm} /$ hour should be admitted as an emergency for specialist assessment. ${ }^{102} 1265$ For patients with moderate or severe IBD not requiring urgent admission, it is important that early contact is made with their IBD clinic, either by the patient or the general practitioner (GP), to ensure that response to therapy is monitored. GPs often prescribe prednisolone, but may be less familiar with increasing the 5-ASA dose to manage flares of UC. An adequate response should be expected within $2-3$ weeks $^{1266}$ and, if not evident, review is required to assess the need to escalate treatment or arrange hospital admission.

A recent study of IBD patients managed in primary care in London showed a high standard of monitoring blood tests and avoidance of unnecessary steroid use, but underprovision of vaccination where recommended by guidelines and overuse of 5-ASA in Crohn's disease. ${ }^{1267}$ A primary care study of UC patients taking 5-ASA showed that dosage, monitoring and adherence were suboptimal, ${ }^{1268}$ and vaccination levels poor. ${ }^{1269}$ Integrated referral pathways have been shown to reduce waiting times for advice and specialist investigations, improve clinical and patient reported outcomes and reduce costs to the healthcare system. ${ }^{1270}$ Internet-based programmes can facilitate structured monitoring for patients being followed up in the community. ${ }^{1271}$ Adherence to medication can be improved by simplifying dose regimens and promoting better understanding and communication between the patient, primary care and IBD clinic team. ${ }^{1272}$ Better education for patients and for community and hospital teams is likely to improve vaccination uptake. ${ }^{1239} 1273-1276$ 
Good Practice Recommendation 33. Integrated care pathways and shared management guidelines should recommend primary care prescribing and monitoring of IBD treatments including mesalazine, thiopurines and methotrexate once patients are in remission on stable dose. Primary care physicians should arrange monitoring blood tests, relevant vaccinations and encourage treatment adherence, supported by regular communication with secondary care (Agreement: $95.7 \%)$.

\section{FUTURE RESEARCH PRIORITIES}

By reviewing the original 414 clinical questions that the guideline development group defined as those facing multidisciplinary clinicians in everyday clinical practice, with comparison to evidence returned from the systematic review process and results from the eDelphi, 20 research priority themes have been determined and are presented in table 12. An essential aspect of any research priority identification process is determination of the importance of this research to those living with UC and Crohn's disease. Accordingly, each research priority theme was drafted in healthcare professional language and for a general audience, with readability testing shown in online supplementary table 4 . Feedback on the importance of each theme was received from two electronic surveys conducted by the UK patient

Box 13 Recommended information to be included in correspondence between secondary and primary care

\section{Information to be included in correspondence from} secondary to primary care:

- Main diagnosis/diagnoses: type and location of IBD and date of diagnosis

- Date(s) of surgery

- Secondary diagnosis/diagnoses-for example, anaemia, vitamin $\mathrm{D}$ deficiency, osteoporosis, extraintestinal manifestations

- Date of last endoscopy with findings and timing of next planned endoscopy

- Date of next planned contact with secondary care

- Current medical therapy including any previous treatments with thiopurines, methotrexate or biologics and reasons for discontinuation

- Recommended length of current medical therapy

- Treatment recommendations in case of a flare: 5-ASA dose increase, prednisolone, budesonide, calcium and vitamin D. Details of who to contact if treatment is initiated in primary care

- Contact details for local IBD team

- Weblink for advice and guidance for primary care (eg, RCGP Spotlight Project toolkit www.rcgp.org.uk/ibd)

Information to be included in correspondence from primary to secondary care:

- Date last prescription issued

- All current and recent medications. Any recent antibiotics

- Number of courses of oral prednisolone issued in last 12 months

- Key results of last blood tests

- Functional impact for example, impact of IBD on employment, family and social functioning

- Any newly diagnosed comorbidities charity Crohn's and Colitis UK (table 12). The 931 respondents to survey 1 and 1448 respondents to survey 2 were anonymous but identified themselves as a UC or Crohn's disease patient, a relative, partner, parent, friend or carer. Subgroup analyses of research priority theme importance are presented in online supplementary table 5. Identified as of particular importance to those living with UC and Crohn's disease were: prevention, risk stratification, diagnosis and management of neoplasia; aetiology and management of pain and fatigue; precision medicine; surgical techniques to minimise postoperative disease recurrence in Crohn's disease; management of non-perianal fistulae in Crohn's disease; and the microbiome including faecal microbial transplantation and the impact of dietary modification in UC. All 20 research priority themes were found to be important to those living with UC and Crohn's disease, with mean scores and confidence intervals for each falling in the upper tertile of the 1-9 Likert scale, with the exception of research regarding improvement of adherence to therapy and the impact on patient outcome. Our findings are complementary to those of the James Lind Alliance and build on the importance of a strong patient voice in research priority setting. ${ }^{1277} 1278$ It is hoped that this exercise will highlight to the academic community, research funders and policy makers where further high-quality research is required to support and advance future clinical practice in UC and Crohn's disease.

\section{CONCLUDING REMARKS}

These British Society of Gastroenterology consensus guidelines on the management of inflammatory bowel disease in adults have been developed along with all major UK stakeholders in UC and Crohn's disease healthcare, including patient representation through close collaboration with Crohn's and Colitis UK.

They are published at a time of rapid change in many aspects of IBD. Recent developments highlighted include new imaging techniques; increasing numbers of new drugs; changes in the way these drugs are used with accelerated treatment and reduction in prolonged use of older therapies with greater toxicity; the increasing importance of infection screening at diagnosis; changes in therapeutic goals (such as mucosal healing) and advances in therapeutic monitoring. This makes IBD treatment ever more complex and highlights the importance of multidisciplinary working, and finding more effective ways to deliver services. We have also emphasised the vital importance of patient education, empowerment and partnership in order to ensure that the patient's needs (and not the technology) remain the focus of all those treating IBD patients.

We have drawn together concise guidance and evidence supporting a wide range of statements that inform best practice in the care of patients with UC and Crohn's disease, including the quality of the evidence available and the strength of recommendations, with a Delphi consensus process to show level of agreement. Many areas are clearly in a state of rapid evolution, and there remain obvious gaps in our understanding. In reference to this, we have presented the key unmet research themes arising from our systematic review and have also presented the importance of each from a patient's perspective. We hope this exercise will highlight aspects of care that are of importance to IBD patients and the healthcare community, and thus may inform government, Research Councils, medical research funding bodies and industry as to areas of research priority in order to advance care for UC and Crohn's disease patients in 2019 and beyond. 
Table 12 Research priority themes identified by guideline development group, with measures of importance determined from two surveys conducted by Crohn's and Colitis UK

Research priority themes regarding both ulcerative colitis and Crohn's disease

1 How do we select the correct treatment, for the correct patient, at the correct time to achieve precision/stratified medicine in clinical practice? What is the comparative efficacy of different classes of biologics in IBD patient subgroups?

With an increasing range of biologic treatments available for Crohn's disease and ulcerative colitis, working out which are better suited for individual patients is more important than ever. How can doctors and other members of the healthcare team select the best treatment at the right time for each patient with IBD? Do some treatments work better or worse in patients with different types of IBD, or with different test results?

2 To achieve treatment to target, is intensification of immunosuppression in an otherwise asymptomatic patient acceptable and in what proportion of patients is mucosal healing an achievable goal?

IBD is less likely to flare up later if the bowel is fully healed. Some patients have no symptoms, but evidence of inflammation is seen during colonoscopy or scans. In these cases, how acceptable is it to increase or change treatment to try and heal this inflammation? How successful and safe is this?

3 What are the most appropriate longitudinal clinical, imaging and endoscopic indices, and what are the best patient reported outcome measures and biomarkers to assess disease activity and monitor response to therapy?

What are the best symptom questionnaires, blood tests, stool (faeces) tests, scans and camera tests (eg, colonoscopy) to determine accurately whether or not IBD is controlled? Which are the best tests to repeat over time to measure change and response to treatment?

4 What is the optimal 6TGN concentration when thiopurines are used as part of combination therapy with a biologic?

Blood tests (to measure 6TGN concentrations) can help doctors to decide on how much azathioprine and mercaptopurine to give to patients for the best chance of benefit and (7.3 to 7.5) at the same time a low risk of side effects. How should these tests be used to get the best effect of these drugs when they are given in combination with newer biologics like infliximab, adalimumab, vedolizumab or ustekinumab?

5 What are the best strategies to prevent, risk stratify, diagnose and manage neoplasia in IBD? What is the optimal strategy for endoscopic surveillance of dysplasia in patients with colitis?

Some people with IBD are at higher risk of developing colon cancer. What is the best way to measure whether someone is at higher risk? What is the best way to prevent diagnose, treat, or look for cancer, or early changes that might develop into cancer? How frequently should patients with IBD be assessed in this way?

6 What causes the symptoms of fatigue and pain in IBD, and what pharmacological and non-pharmacological therapies are beneficial?

Why do many IBD patients experience fatigue (tiredness not relieved by resting) and pain? What treatments (drugs or non-drug treatments) are best to help these symptoms? Why do these symptoms continue even if their Crohn's disease or ulcerative colitis are no longer active?

7 What is the optimal operative technique to maintain fertility, fecundity, sexual function and continence in IBD patients undergoing surgery? Some operations for Crohn's disease and ulcerative colitis can have an impact on sexual intercourse, fertility, pregnancy and bladder/bowel function. What are the best techniques doctors can carry out during surgery to minimise this happening in men and women?

8 What are the most effective strategies to empower patients towards effective self-management? How should patients' views, beliefs and attitude to risk best be considered when making treatment decisions in IBD? In young adults can this be supported by dedicated transition models from paediatric to adult IBD services?

Self-management in IBD refers to the practice of giving patients enough knowledge and support to manage their disease themselves most of the time. This can reduce the number of visits to the hospital, and patients can feel more in control. What is the best way to educate and empower patients to manage their own condition? How can the needs, views, wishes and feelings of each patient be included in decisions about their treatment? Does the approach need to be different for different groups of patients, including young adults moving from the children's hospital service to the adult hospital service?

9 Should EBV serology influence immunomodulatory therapy algorithms in subgroups of patients with IBD?

Epstein Barr virus (EBV) causes glandular fever, which usually affects children and young people. Many catch the virus without any symptoms at all and may carry it without knowing. Patients who carry the EBV virus can, very rarely, develop severe complications including cancer of the lymph glands when treated with some drugs used in IBD (particularly azathioprine or mercaptopurine). Should knowing about whether an IBD patient has previously had EBV be used to decide the type of treatment they receive?

10 Is it safe and efficacious to administer live vaccination to patients on 'low dose' immunosuppression-for example, thiopurines and methotrexate? Live vaccinations can theoretically cause infections in individuals with a suppressed immune system. Examples include shingles and yellow fever. This means that live vaccinations are often avoided if patients are taking immunosuppressants. Patients who have been recently vaccinated may not be started on treatments to suppress the immune system for many weeks or months to give time for the vaccination to take effect and for the risk of infection to reduce. This delay can cause problems for patients who are very ill. Is it safe to give live vaccines to patients who are receiving immunosuppressant drugs such as azathioprine, methotrexate or low doses of the steroid prednisolone?

11 What methods can improve adherence with therapy in IBD, and does this improve patient outcome?

Some patients find it difficult to remember to take their medications, particularly if they feel well and have to take the treatments over a long period of time. How can patients be helped to remember to take their treatments regularly on a long-term basis? How much additional benefit will they get from taking their treatment most or all of the time?

12 What is the safety profile of vedolizumab, ustekinumab and tofacitinib in pregnancy and during breastfeeding? How safe are the drugs vedolizumab, ustekinumab, and tofacitinib (all relatively new drugs used in IBD) for the fetus and mother during pregnancy? How safe are these drugs for the baby following birth if breastfeeding?

13 Can shared care algorithms between primary and secondary care improve outcomes for patients and make delivery of care more cost effective? Systems of 'shared care' involve sharing of patient treatment plans between hospitals and GPs. They specify which drugs should be used and how treatment should be monitored. Do these help patients have better control of their disease? In addition, do shared care systems reduce the overall costs of treatment for the NHS?

Research priority themes regarding ulcerative colitis only

14 What is the optimal induction dose and drug administration interval for infliximab in acute severe ulcerative colitis, and how do we stratify this for the individual patient?

Some patients with ulcerative colitis have to be admitted to hospital for a severe flare. Standard treatment is with intravenous steroids (via injection into a vein). If this treatment doesn't work, doctors sometimes use infliximab (a biologic drug) to treat them. When should infliximab be used and what is the best dose to give? Should the treatment be tailored to suit each individual patient's condition, and what symptoms or tests are best used to decide this?

15 Which bacteria confer benefit in ulcerative colitis as probiotics or within faecal microbial transplantation, and is this patient-specific? Can dietary intervention 7.8 improve patient outcome through alteration of the microbiome or other mechanisms?

Mean theme

importance to $\mathrm{n}=931$ survey

respondents

(95\% Cl):

$\min =1 \max =9$

7.9

(7.8 to 8.0)

7.2

$$
8.1
$$

(8.0 to 8.2$)$

7.9

Within the gut there is bacteria that may have an impact on ulcerative colitis, and altering this could reduce the inflammation caused by this condition. Which bacteria that

lives in the gut can be changed and how can this be achieved (for example, by giving treatments containing types of bacteria, or giving bacteria from someone else's bowel as

a treatment). Are different bacteria important for different patients? Can changes to diet alter the bacteria in the gut and if so, is this beneficial?

Research priority themes regarding Crohn's disease only

16 What is the optimal treatment strategy for mild Crohn's disease? 
Table 12 Continued

\begin{tabular}{|c|c|c|}
\hline & & $\begin{array}{l}\text { Mean theme } \\
\text { importance to } \\
\mathrm{n}=931 \text { survey } \\
\text { respondents } \\
(95 \% \mathrm{Cl}): \\
\min =1 \max =9\end{array}$ \\
\hline 17 & $\begin{array}{l}\text { What is the role of diet and nutrition (ie, oral, enteral and parenteral) in inducing and maintaining remission of adult Crohn's disease? What is the mechanism } \\
\text { of action of existing nutritional approaches to achieving remission in IBD? } \\
\text { Dietary treatment may involve changing what we eat, adding dietary supplements or replacing everything we eat with special nutritional products designed to provide } \\
\text { complete nutrition. These are likely to be in the form of milkshake-type drinks. Some patients also receive an intravenous diet, which means a tube provides nutrients directly } \\
\text { into their blood stream. How effective is dietary treatment in controlling Crohn's disease in adults? How effective is it in preventing Crohn's disease from flaring after it has } \\
\text { been fully controlled? How do these treatments work to heal the inflamed gut in Crohn's disease? }\end{array}$ & $\begin{array}{l}7.5 \\
\text { (7.3 to } 7.6 \text { ) }\end{array}$ \\
\hline 18 & $\begin{array}{l}\text { What are the optimal techniques for resection and anastomosis during surgery for Crohn's disease to minimise disease recurrence? } \\
\text { When patients with Crohn's disease have an operation to remove part of the small or large bowel, the two ends of bowel are often joined back together again. How this is } \\
\text { done can influence the risk of disease coming back. What is the most effective surgical technique to minimise recurrence? }\end{array}$ & $\begin{array}{l}8.0 \\
\text { (7.9 to } 8.1)\end{array}$ \\
\hline \multirow[t]{2}{*}{19} & $\begin{array}{l}\text { What are the best methods to quantify fibrosis in Crohn's disease strictures? } \\
\text { Patients with Crohn's disease sometimes develop narrowed, scarred areas in the bowel, particularly in the small intestine. These are called strictures and can eventually lead } \\
\text { to blockages. While current drugs can control the inflammation found in Crohn's disease, they are not good at reversing this scarring process. What is the best way to measure } \\
\text { how much scarring there is in areas of narrowed bowel? }\end{array}$ & $\begin{array}{l}7.7 \\
\text { (7.6 to } 7.8 \text { ) }\end{array}$ \\
\hline & & $\begin{array}{l}\text { Mean theme } \\
\text { importance to } \\
\mathrm{n}=1448 \text { survey } \\
\text { respondents } \\
(95 \% \mathrm{Cl}): \\
\min =1 \max =9\end{array}$ \\
\hline $20^{*}$ & $\begin{array}{l}\text { Is there an optimal management strategy for non-perianal fistulae in Crohn's disease?* } \\
\text { A fistula in Crohn's disease is when a deep ulcer in the bowel forms a connection or track with another part of the body. They can lead to infection or abscesses. Perianal } \\
\text { fistulas are when tracks link the rectum (lower bowel) with the skin around the anus. Non-perianal fistulas involve connections or tracks from the bowel to other structures } \\
\text { like the bladder, the vagina, the skin of the tummy wall, or tracks running from one part of the bowel to another. There is less evidence about how to treat non-perianal fistulas } \\
\text { with medication or surgery. What are the best treatments and combinations of treatments for Crohn's disease when it causes non-perianal fistulas?* }\end{array}$ & $\begin{array}{l}8.0 \\
\text { (7.9 to } 8.0)\end{array}$ \\
\hline
\end{tabular}

Twenty research priority themes identified by the guideline development group are presented in healthcare professional language (plain text), with corresponding general audience versions (italics). Mean scores of importance, judged on a Likert scale of 1-9 (1='not important' and 9='very important') are shown with $95 \%$ Cls, determined from two electronic surveys conducted by Crohn's and Colitis UK. *Feedback regarding research priority themes 1-19 was the subject of the first survey from Crohn's and Colitis UK, and research priority 20 was the subject of the second survey. Therefore, comparisons of the relative mean theme importance should not be made between themes 1-19 and theme 20 as they represent a potentially different cohort of respondents.

\section{Author affiliations}

${ }^{1}$ Newcastle University, Newcastle upon Tyne, UK

${ }^{2}$ Newcastle Upon Tyne Hospitals NHS Foundation Trust, Newcastle Upon Tyne, UK

${ }^{3}$ Royal Devon and Exeter NHS Foundation Trust, Exeter, UK

${ }^{4}$ University of Exeter, Exeter, UK

${ }^{5}$ Cambridge University Hospitals NHS FoundationTrust, Cambridge, UK

${ }^{6}$ Chelsea and Westminster Hospital NHS Foundation Trust, London, UK

${ }^{7}$ Imperial College London, London, UK

${ }^{8}$ Royal Liverpool and Broadgreen University Hospitals NHS Trust, Liverpool, UK

${ }^{9}$ The Pennine Acute Hospitals NHS Trust, Manchester, UK

${ }^{10}$ University of Manchester, Manchester, UK

${ }^{11}$ King's College Hospital NHS Foundation Trust, London, UK

${ }^{12}$ King's College London, London, UK

${ }^{13}$ Guy's and St Thomas' NHS Foundation Trust, London, UK

${ }^{14}$ Barts Health NHS Trust, London, UK

${ }^{15}$ Barts and the London School of Medicine and Dentistry, London, UK

${ }^{16}$ Leeds Teaching Hospitals NHS Trust, Leeds, UK

${ }^{17}$ University of Leeds, Leeds, UK

${ }^{18}$ New Road Surgery, Rickmansworth, UK

${ }^{19}$ University of Cambridge, Cambridge, UK

${ }^{20}$ Systematic Research Ltd, Quorn, UK

${ }^{21}$ Royal College of Surgeons in Ireland (RCSI), Dublin, Ireland

${ }^{22}$ ECD Solutions, Bridgetown, Barbados

${ }^{23}$ University of Edinburgh, Edinburgh, UK

${ }^{24}$ Western General Hospital, Edinburgh, UK

${ }^{25}$ St Mark's Hospital, Harrow, UK

${ }^{26}$ University Hospitals Bristol NHS Foundation Trust, Bristol, UK

${ }^{27}$ Royal Hospital for Children Glasgow, Glasgow, UK

${ }^{28} \mathrm{Crohn's}$ and Colitis UK, Hatfield, UK

${ }^{29}$ Glasgow Royal Infirmary, Glasgow, UK

${ }^{30}$ University of Glasgow, Glasgow, UK

${ }^{31}$ Queen Elizabeth Hospital Birmingham NHSFoundation Trust, Birmingham, UK

${ }^{32}$ University of Birmingham, Birmingham, UK

${ }^{33}$ University College London, London, UK

${ }^{34}$ University College London Hospitals NHS Foundation Trust, London, UK

${ }^{35}$ Brighton and Sussex University Hospitals NHS Trust, Brighton, UK

${ }^{36}$ Brighton and Sussex Medical School, Brighton, UK

${ }^{37}$ Royal Wolverhampton NHS Trust, Wolverhampton, UK

${ }^{38}$ University of Wolverhampton, Wolverhampton, UK

${ }^{39}$ University Hospital of Wales, Cardiff, UK
Acknowledgements We are grateful to the Clinical Standards and Services Committee of the British Society of Gastroenterology for commissioning this guidance, and to the following Stakeholders and Societies who have provided valuable review and comment during the guideline development process: British Society of Gastroenterology, Crohn's and Colitis UK, Association of Coloproctology of Great Britain and Ireland, Royal College of Nursing, British Society of Paediatric Gastroenterology, Hepatology and Nutrition, British Dietetic Association, British Society of Gastrointestinal and Abdominal Radiology, Primary Care Society for Gastroenterology and IBD UK. We are grateful to Sophie Bassil, Mitesh Gandhi and the wider Crohn's and Colitis UK team for conducting two electronic surveys regarding the importance of research priority themes identified by the guideline development process, and to the patients, their relatives, partners, parents, friends and carers for completing these surveys. We are grateful to Professor Juliet Compston, Professor Emeritus of Bone Medicine, Cambridge Biomedical Campus, UK for peer review of our osteoporosis prevention and management in IBD figure. The guideline development process was funded by the British Society of Gastroenterology. CAL is a Clinical Lecturer supported by the NIHR.

Collaborators IBD guidelines eDelphi consensus group: Ian Arnott; Kevin I Barrett; R Mark Beattie; Stuart Bloom; Keith Bodger; Richard R Brady; Matthew J Brookes; Steven R Brown; Jeffrey R Butterworth; Christopher R Calvert; Rachel Campbell; Tom Creed; Nicholas M Croft; Fraser Cummings; R Justin Davies; David Devadason; Anjan Dhar; Chris Dipper; Julie Duncan; Malcolm Dunlop; Dharmaraj Durai; Martyn D Evans; Omar Faiz; Nicola S Fearnhead; Alexander C Ford; Aileen Fraser; Vikki Garrick; Daniel R Gaya; James Goodhand; Nigel Hall; Richard Hansen; Marcus Harbord; A Barney Hawthorne; Bu'Hussain Hayee; Sarah Hearnshaw; Paul Henderson; Philip Hendy; Tariq Iqbal; Paul D Johnston; Nicholas A Kennedy; Christopher A Lamb; Jimmy K Limdi; James $\mathrm{O}$ Lindsay; Alan J Lobo; Miranda Lomer; Richard E Lovegrove; John C Mansfield; Simon Mclaughlin; Christopher G Mountford; Rafeeq Muhammed; Nurulamin M Noor; Simon Panter; Gareth C Parkes; Miles Parkes; Thomas D Pinkney; Chris Probert; Tim Raine; Richard K Russell; Matthew D Rutter; Mark A Samaan; Jeremy D Sanderson; Christian P Selinger; Ashit Shah; Steve J Short; Baljit Singh; Philip J Smith; Melissa Smith; R Alexander Speight; Catherine Stansfield; Helen Steed; Stuart A Taylor; Helen Terry; Tony C Tham; Nick P Thompson; Gareth I Walker; Catherine L Wall; Janindra Warusavitarne; Gethin L Williams; R Graeme Wilson; David C Wilson; Lisa C Younge. See online supplementary table 1 for affiliations of the IBD guidelines eDelphi consensus group.

Contributors The guideline development group forms the authorship of this manuscript. All fulfil the ICMJE recommendations for authorship with appropriate involvement at all required stages of the guideline development process which followed GRADE methodology as described. All have contributed to manuscript 
drafting. The additional named contributors formed the IBD guidelines eDelphi consensus group, contributing to voting over three rounds of Delphi to develop the statements and good practice recommendations set out in this guideline.

Funding The authors have not declared a specific grant for this research from any funding agency in the public, commercial or not-for-profit sectors.

Competing interests Conflicts of interest for authors and contributors are presented in online supplementary table 2.

Patient consent for publication Not required.

Provenance and peer review Commissioned; externally peer reviewed.

Open access This is an open access article distributed in accordance with the Creative Commons Attribution Non Commercial (CC BY-NC 4.0) license, which permits others to distribute, remix, adapt, build upon this work noncommercially, and license their derivative works on different terms, provided the original work is properly cited, appropriate credit is given, any changes made indicated, and the use is non-commercial. See: http://creativecommons.org/ licenses/by-nc/4.0/.

\section{ORCID iDs}

Christopher Andrew Lamb https://orcid.org/0000-0002-7271-4956

Nicholas A Kennedy https://orcid.org/0000-0003-4368-1961

Tim Raine https://orcid.org/0000-0002-5855-9873

Philip Anthony Hendy https://orcid.org/0000-0002-1377-717

Philip J Smith https://orcid.org/0000-0003-1568-3978

Jimmy K Limdi https://orcid.org/0000-0002-1039-6251

Bu'Hussain Hayee https://orcid.org/0000-0003-1670-8815

Miranda C E Lomer https://orcid.org/0000-0002-9369-8115

Gareth C Parkes https://orcid.org/0000-0002-5285-7714

Christian Selinger https://orcid.org/0000-0003-2022-5859

Kevin J Barrett https://orcid.org/0000-0002-0785-1815

R Justin Davies https://orcid.org/0000-0001-6904-1255

Cathy Bennett https://orcid.org/0000-0002-9112-5698

Stuart Gittens https://orcid.org/0000-0001-7137-5799

Malcolm G Dunlop https://orcid.org/0000-0002-3033-5851

Omar Faiz https://orcid.org/0000-0003-1117-1588

Aileen Fraser https://orcid.org/0000-0001-6462-5091

Vikki Garrick https://orcid.org/0000-0001-8643-8617

Paul D Johnston https://orcid.org/0000-0002-4192-6526

Miles Parkes https://orcid.org/0000-0002-6467-0631

Jeremy Sanderson https://orcid.org/0000-0002-0556-6965

Helen Terry https://orcid.org/0000-0002-8920-5752

Daniel R Gaya https://orcid.org/0000-0003-1942-7568

Tariq H lqbal https://orcid.org/0000-0002-6681-9882

Stuart A Taylor https://orcid.org/0000-0002-6765-8806

Melissa Smith https://orcid.org/0000-0002-7442-6093

Matthew Brookes https://orcid.org/0000-0002-8782-0292

Richard Hansen https://orcid.org/0000-0002-3944-6646

A Barney Hawthorne https://orcid.org/0000-0002-8768-4550

\section{REFERENCES}

1 Mowat C, Cole A, Windsor A, et al. Guidelines for the management of inflammatory bowel disease in adults. Gut 2011;60:571-607.

2 Guyatt GH, Oxman AD, Vist GE, et al. GRADE: an emerging consensus on rating quality of evidence and strength of recommendations. BMJ 2008;336:924-6.

3 Brouwers MC, Kho ME, Browman GP, et al. AGREE II: advancing guideline development, reporting and evaluation in health care. CMAJ 2010;182:E83 9-E842.

4 BSG Guidelines Advice Document. British Society of Gastroenterology. 2018. https://www.bsg.org.uk/clinical/bsg-guidelines/guideline-development-within-thebsg-cssc-policies.html (Accessed 1 Feb 2019).

5 Brown SR, Fearnhead NS, Faiz OD, et al. The Association of Coloproctology of Great Britain and Ireland consensus guidelines in surgery for inflammatory bowel disease. Colorectal Dis 2018;20(Suppl 8):3-117.

6 Harris PA, Taylor R, Thielke R, et al. Research electronic data capture (REDCap)-a metadata-driven methodology and workflow process for providing translational research informatics support. J Biomed Inform 2009;42:377-81.

7 Höie 0 , Wolters F, Riis L, et al. Ulcerative colitis: patient characteristics may predict 10 -yr disease recurrence in a European-wide population-based cohort. Am J Gastroenterol 2007;102:1692-701.

8 Langholz E, Munkholm P, Davidsen M, et al. Course of ulcerative colitis: analysis of changes in disease activity over years. Gastroenterology 1994;107:3-11.

9 Leighton JA, Shen B, Baron TH, et al. ASGE guideline: endoscopy in the diagnosis and treatment of inflammatory bowel disease. Gastrointest EndosC 2006;63:558-65
10 Park SH, Yang SK, Park SK, et al. Atypical distribution of inflammation in newly diagnosed ulcerative colitis is not rare. Can J Gastroenterol Hepatol 2014;28:125-30

11 Kim B, Barnett JL, Kleer CG, et al. Endoscopic and histological patchiness in treated ulcerative colitis. Am J Gastroenterol 1999;94:3258-62.

12 Odze R, Antonioli D, Peppercorn M, et al. Effect of topical 5-aminosalicylic acid (5-ASA) therapy on rectal mucosal biopsy morphology in chronic ulcerative colitis. Am J Surg Pathol 1993;17:869-75.

13 Abdelrazeq AS, Wilson TR, Leitch DL, et al. Ileitis in ulcerative colitis: is it a backwash? Dis Colon Rectum 2005:48:2038-46.

14 Feakins RM. British Society of Gastroenterology. Inflammatory bowel disease biopsies: updated British Society of Gastroenterology reporting guidelines. J Clin Pathol 2013;66:1005-26.

15 Tremaine WJ. Review article: Indeterminate colitis-definition, diagnosis and management. Aliment Pharmacol Ther 2007;25:13-17.

16 Price $A B$. Overlap in the spectrum of non-specific inflammatory bowel disease'colitis indeterminate'. J Clin Pathol 1978:31:567-77.

17 Guindi M, Riddell RH. Indeterminate colitis. J Clin Pathol 2004;57:1233-44.

18 Prenzel F, Uhlig $\mathrm{HH}$. Frequency of indeterminate colitis in children and adults with IBD - a metaanalysis. J Crohns Colitis 2009;3:277-81.

19 Romberg-Camps MJ, Dagnelie PC, Kester AD, et al. Influence of phenotype at diagnosis and of other potential prognostic factors on the course of inflammatory bowel disease. Am J Gastroenterol 2009:104:371-83.

20 Witte J, Shivananda S, Lennard-Jones JE, et al. Disease outcome in inflammatory bowel disease: mortality, morbidity and therapeutic management of a 796-person inception cohort in the European Collaborative Study on Inflammatory Bowe Disease (EC-IBD). Scand J Gastroentero/ 2000;35:1272-7.

21 Moum B, Ekbom A, Vatn MH, et al. Inflammatory bowel disease: re-evaluation of the diagnosis in a prospective population based study in south eastern Norway. Gut 1997:40:328-32

22 Satsangi J, Silverberg MS, Vermeire S, et al. The Montreal classification of inflammatory bowel disease: controversies, consensus, and implications. Gut 2006:55:749-53.

23 Levine A, Griffiths A, Markowitz J, et al. Pediatric modification of the Montreal classification for inflammatory bowel disease: the Paris classification. Inflamm Bowel Dis 2011;17:1314-21.

24 Spekhorst LM, Visschedijk MC, Alberts R, et al. Performance of the Montreal classification for inflammatory bowel diseases. World J Gastroenterol 2014;20:15374-81.

25 Gower-Rousseau C, Dauchet L, Vernier-Massouille G, et al. The natural history of pediatric ulcerative colitis: a population-based cohort study. Am J Gastroenterol 2009;104:2080-8.

26 Fumery M, Singh S, Dulai PS, et al. Natural history of adult ulcerative colitis in population-based cohorts: a systematic review. Clin Gastroenterol Hepatol 2018;16:343-56.

27 Katsanos KH, Vermeire S, Christodoulou DK, et al. Dysplasia and cancer in inflammatory bowel disease 10 years after diagnosis: results of a population-based European collaborative follow-up study. Digestion 2007;75:113-21.

28 Rutter MD, Saunders BP, Wilkinson KH, et al. Cancer surveillance in longstanding ulcerative colitis: endoscopic appearances help predict cancer risk. Gut 2004;53:1813-6.

29 Silverberg MS, Satsangi J, Ahmad T, et al. Toward an integrated clinical, molecular and serological classification of inflammatory bowel disease: report of a Working Party of the 2005 Montreal World Congress of Gastroenterology. Can J Gastroentero/ 2005;19(Suppl A):5A-36.

30 Langholz E, Munkholm P, Davidsen M, et al. Changes in extent of ulcerative colitis: a study on the course and prognostic factors. Scand I Gastroenterol 1996;31:260-6

31 Henriksen M, Jahnsen J, Lygren I, et al. Ulcerative colitis and clinical course: results of a 5-year population-based follow-up study (the IBSEN study). Inflamm Bowel Dis 2006:12:543-50.

32 Schroeder KW, Tremaine WJ, Ilstrup DM. Coated oral 5-aminosalicylic acid therapy for mildly to moderately active ulcerative colitis. A randomized study. N Eng/ J Med 1987:317:1625-9.

33 Lewis JD, Chuai S, Nessel L, et al. Use of the noninvasive components of the Mayo score to assess clinical response in ulcerative colitis. Inflamm Bowel Dis 2008; 14:1660-6

34 Thia KT, Loftus EV, Pardi DS, et al. Measurement of disease activity in ulcerative colitis: interobserver agreement and predictors of severity. Inflamm Bowel Dis 2011:17:1257-64

35 Travis SP, Schnell D, Krzeski P, et al. Developing an instrument to assess the endoscopic severity of ulcerative colitis: the Ulcerative Colitis Endoscopic Index of Severity (UCEIS). Gut 2012;61:535-42.

36 Travis SP, Schnell D, Krzeski P, et al. Reliability and initial validation of the ulcerative colitis endoscopic index of severity. Gastroenterology 2013;145:987-95. 
37 Lobatón T, Bessissow T, De Hertogh G, et al. The Modified Mayo Endoscopic Score (MMES): a new index for the assessment of extension and severity of endoscopic activity in ulcerative colitis patients. J Crohns Colitis 2015;9:846-52.

38 Lee JS, Kim ES, Moon W. Chronological review of endoscopic indices in inflammatory bowel disease. Clin Endosc 2019;52:129-36.

39 Mohammed Vashist N, Samaan M, Mosli MH, et al. Endoscopic scoring indices for evaluation of disease activity in ulcerative colitis. Cochrane Database Syst Rev 2018;1:CD011450.

40 Peyrin-Biroulet L, Panés J, Sandborn WJ, et al. Defining disease severity in inflammatory bowel diseases: current and future directions. Clin Gastroenterol Hepatol 2016;14:348-54.

41 De Boer M, Grootenhuis M, Derkx B, et al. Health-related quality of life and psychosocial functioning of adolescents with inflammatory bowel disease. Inflamm Bowel Dis 2005;11:400-6.

42 Bojic D, Bodger K, Travis S. Patient Reported Outcome Measures (PROMs) in inflammatory bowel disease: new data. J Crohns Colitis 2017;11(Suppl 2): $: 576-85$

43 Higgins PD, Schwartz M, Mapili J, et al. Patient defined dichotomous end points for remission and clinical improvement in ulcerative colitis. Gut 2005;54:782-8.

44 Christensen B, Rubin DT. Understanding endoscopic disease activity in IBD: how to incorporate it into practice. Curr Gastroenterol Rep 2016;18:5.

45 Samaan MA, Mosli MH, Sandborn WJ, et al. A systematic review of the measurement of endoscopic healing in ulcerative colitis clinical trials: recommendations and implications for future research. Inflamm Bowel Dis 2014;20:1465-71

46 Neurath MF, Travis SP. Mucosal healing in inflammatory bowel diseases: a systematic review. Gut 2012;61:1619-35.

47 Bryant RV, Costello SP, Schoeman S, et al. Limited uptake of ulcerative colitis "treat-to-target" recommendations in real-world practice. J Gastroenterol Hepatol 2018;33:599-607.

48 Bryant RV, Winer S, Travis SP, et al. Systematic review: histological remission in inflammatory bowel disease. Is 'complete' remission the new treatment paradigm? An IOIBD initiative. J Crohns Colitis 2014;8:1582-97.

49 Mazzuoli S, Guglielmi FW, Antonelli E, et al. Definition and evaluation of mucosal healing in clinical practice. Dig Liver Dis 2013;45:969-77.

50 Frøslie KF, Jahnsen J, Moum BA, et al. Mucosal healing in inflammatory bowel disease: results from a Norwegian population-based cohort. Gastroenterology 2007;133:412-22

51 Meucci G, Fasoli R, Saibeni S, et al. Prognostic significance of endoscopic remission in patients with active ulcerative colitis treated with oral and topical mesalazine: a prospective, multicenter study. Inflamm Bowel Dis 2012;18:1006-10.

52 Ardizzone S, Cassinotti A, Duca P, et al. Mucosal healing predicts late outcomes after the first course of corticosteroids for newly diagnosed ulcerative colitis. Clin Gastroenterol Hepatol 2011;9:483-9.

53 Colombel JF, Rutgeerts P, Reinisch W, et al. Early mucosal healing with infliximab is associated with improved long-term clinical outcomes in ulcerative colitis. Gastroenterology 2011;141:1194-201.

54 Walsh A, Palmer R, Travis S. Mucosal healing as a target of therapy for colonic inflammatory bowel disease and methods to score disease activity. Gastrointest Endosc Clin N Am 2014;24:367-78.

55 Saxena AP, Limdi JK, Farraye FA. Zeroing in on endoscopic and histologic mucosal healing to reduce the risk of colorectal neoplasia in inflammatory bowel disease. Gastrointest Endosc 2017;86:1012-4.

56 Peyrin-Biroulet L, Sandborn W, Sands BE, et al. Selecting Therapeutic Targets in Inflammatory Bowel Disease (STRIDE): Determining Therapeutic Goals for Treat-toTarget. Am J Gastroenterol 2015;110:1324-38.

57 Feagan BG, Macdonald JK. Oral 5-aminosalicylic acid for induction of remission in ulcerative colitis. Cochrane Database Syst Rev 2012;10:CD000543.

58 Ford AC, Kane SV, Khan KJ, et al. Efficacy of 5-aminosalicylates in Crohn's disease: systematic review and meta-analysis. Am J Gastroenterol 2011;106:617-29.

59 Wang Y, Parker CE, Bhanji T, et al. Oral 5-aminosalicylic acid for induction of remission in ulcerative colitis. Cochrane Database Syst Rev 2016;4:CD000543.

60 Ford AC, Achkar JP, Khan KJ, et al. Efficacy of 5-aminosalicylates in ulcerative colitis: systematic review and meta-analysis. Am J Gastroenterol 2011;106:601-16.

61 Hanauer SB, Sandborn WJ, Dallaire C, et al. Delayed-release oral mesalamine $4.8 \mathrm{~g} /$ day ( $800 \mathrm{mg}$ tablets) compared to $2.4 \mathrm{~g} /$ day ( $400 \mathrm{mg}$ tablets) for the treatment of mildly to moderately active ulcerative colitis: The ASCEND I trial. Can I Gastroenterol 2007;21:827-34.

62 Hanauer SB, Sandborn WJ, Kornbluth A, et al. Delayed-release oral mesalamine at $4.8 \mathrm{~g} /$ day ( $800 \mathrm{mg}$ tablet) for the treatment of moderately active ulcerative colitis: the ASCEND II trial. Am J Gastroentero/ 2005;100:2478-85.

63 Sandborn WJ, Regula J, Feagan BG, et al. Delayed-release oral mesalamine 4.8 $\mathrm{g} / \mathrm{day}$ (800-mg tablet) is effective for patients with moderately active ulcerative colitis. Gastroenterology 2009;137:1934-43.
64 Orchard TR, van der Geest SA, Travis SP. Randomised clinical trial: early assessment after 2 weeks of high-dose mesalazine for moderately active ulcerative colitis - new light on a familiar question. Aliment Pharmacol Ther 2011;33:1028-35.

65 Lichtenstein GR, Ramsey D, Rubin DT. Randomised clinical trial: delayed-release oral mesalazine $4.8 \mathrm{~g} /$ day vs. $2.4 \mathrm{~g} /$ day in endoscopic mucosal healing-ASCEND I and II combined analysis. Aliment Pharmacol Ther 2011;33:672-8.

66 Kamm MA, Lichtenstein GR, Sandborn WJ, et al. Effect of extended MMX mesalamine therapy for acute, mild-to-moderate ulcerative colitis. Inflamm Bowel Dis 2009;15:1-8.

67 Osterman MT, Aberra FN, Cross R, et al. Mesalamine dose escalation reduces fecal calprotectin in patients with quiescent ulcerative colitis. Clin Gastroenterol Hepatol 2014;12:1887-93.

68 Pruitt R, Hanson J, Safdi M, et al. Balsalazide is superior to mesalamine in the time to improvement of signs and symptoms of acute mild-to-moderate ulcerative colitis. Am J Gastroenterol 2002;97:3078-86.

69 Levine DS, Riff DS, Pruitt R, et al. A randomized, double blind, dose-response comparison of balsalazide $(6.75 \mathrm{~g})$, balsalazide $(2.25 \mathrm{~g})$, and mesalamine $(2.4 \mathrm{~g})$ in the treatment of active, mild-to-moderate ulcerative colitis. Am J Gastroenterol 2002;97:1398-407.

70 Lichtenstein GR, Kamm MA, Boddu P, et al. Effect of once- or twice-daily MMX mesalamine (SPD476) for the induction of remission of mild to moderately active ulcerative colitis. Clin Gastroenterol Hepatol 2007:5:95-102.

71 Mansfield JC, Giaffer MH, Cann PA, et al. A double-blind comparison of balsalazide, $6.75 \mathrm{~g}$, and sulfasalazine, $3 \mathrm{~g}$, as sole therapy in the management of ulcerative colitis. Aliment Pharmacol Ther 2002;16:69-77.

72 Probert CS, Dignass AU, Lindgren S, et al. Combined oral and rectal mesalazine for the treatment of mild-to-moderately active ulcerative colitis: rapid symptom resolution and improvements in quality of life. J Crohns Colitis 2014;8:200-7.

73 Marteau P, Probert CS, Lindgren S, et al. Combined oral and enema treatment with Pentasa (mesalazine) is superior to oral therapy alone in patients with extensive mild/moderate active ulcerative colitis: a randomised, double blind, placebo controlled study. Gut 2005;54:960-5.

74 Ford AC, Khan KJ, Achkar JP, et al. Efficacy of oral vs. topical, or combined oral and topical 5-aminosalicylates, in ulcerative colitis: systematic review and meta-analysis. Am J Gastroenterol 2012;107:167-76.

75 Frieri G, Pimpo M, Galletti B, et al. Long-term oral plus topical mesalazine in frequently relapsing ulcerative colitis. Dig Liver Dis 2005;37:92-6.

76 Oikonomou K, Kapsoritakis A, Eleftheriadis T, et al. Renal manifestations and complications of inflammatory bowel disease. Inflamm Bowel Dis 2011;17:1034-45.

77 Patel H, Barr A, Jeejeebhoy KN. Renal effects of long-term treatment with 5-aminosalicylic acid. Can J Gastroenterol 2009;23:170-6.

78 Oikonomou KA, Kapsoritakis AN, Stefanidis I, et al. Drug-induced nephrotoxicity in inflammatory bowel disease. Nephron Clin Pract 2011;119:889-94.

79 Molnár T, Farkas K, Nagy F, et al. Sulfasalazine-induced nephrotic syndrome in a patient with ulcerative colitis. Inflamm Bowel Dis 2010;16:552-3.

80 Fardy JM, Lloyd DA, Reynolds RP. Adverse effects with oral 5-aminosalicyclic acid. J Clin Gastroenterol 1988:10:635-7.

81 Heap GA, So K, Weedon M, et al. Clinical features and HLA association of 5-aminosalicylate (5-ASA)-induced nephrotoxicity in inflammatory bowel disease. J Crohns Colitis 2016;10:149-58.

82 Muller AF, Stevens PE, McIntyre AS, et al. Experience of 5-aminosalicylate nephrotoxicity in the United Kingdom. Aliment Pharmacol Ther 2005;21:1217-24.

83 Gisbert JP, Luna M, González-Lama Y, et al. Effect of 5-aminosalicylates on renal function in patients with inflammatory bowel disease: 4-year follow-up study. Gastroenterol Hepatol 2008;31:477-84.

84 Lennard-Jones JE, Longmore AJ, Newell AC, et al. An assessment of prednisone, salazopyrin, and topical hydrocortisone hemisuccinate used as out-patient treatment for ulcerative colitis. Gut 1960;1:217-22.

85 Truelove SC, Watkinson G, Draper G. Comparison of corticosteroid and sulphasalazine therapy in ulcerative colitis. Br Med J 1962;2:1708-11.

86 D'Haens GR, Kovács A, Vergauwe P, et al. Clinical trial: Preliminary efficacy and safety study of a new Budesonide-MMX® $9 \mathrm{mg}$ extended-release tablets in patients with active left-sided ulcerative colitis. J Crohns Colitis 2010;4:153-60.

87 Sandborn WJ, Travis S, Moro L, et al. Once-daily budesonide MMX® extendedrelease tablets induce remission in patients with mild to moderate ulcerative colitis: results from the CORE I study. Gastroenterology 2012;143:1218-26.

88 Travis SP, Danese S, Kupcinskas L, et al. Once-daily budesonide MMX in active, mild-to-moderate ulcerative colitis: results from the randomised CORE II study. Gut 2014:63:433-41.

89 Sherlock ME, MacDonald JK, Griffiths AM, et al. Oral budesonide for induction of remission in ulcerative colitis. Cochrane Database Syst Rev 2015:CD007698.

90 Sandborn WJ, Danese S, D'Haens G, et al. Induction of clinical and colonoscopic remission of mild-to-moderate ulcerative colitis with budesonide MMX $9 \mathrm{mg}$ : pooled analysis of two phase 3 studies. Aliment Pharmacol Ther 2015;41:409-18. 
91 Rubin DT, Cohen RD, Sandborn WJ, et al. OP011. Budesonide MMX® 9 mg for inducing remission in patients with mild-to-moderate ulcerative colitis not adequately controlled with oral 5-ASAs. J Crohns Colitis 2015;9(Suppl 1):S7.

92 Danese S, Siegel CA, Peyrin-Biroulet L. Review article: integrating budesonideMMX into treatment algorithms for mild-to-moderate ulcerative colitis. Aliment Pharmacol Ther 2014;39:1095-103.

93 Löfberg R, Danielsson A, Suhr O, et al. Oral budesonide versus prednisolone in patients with active extensive and left-sided ulcerative colitis. Gastroenterology 1996:110:1713-8.

94 Schoon EJ, Bollani S, Mills PR, et al. Bone mineral density in relation to efficacy and side effects of budesonide and prednisolone in Crohn's disease. Clin Gastroenterol Hepatol 2005;3:113-21.

95 Gross V, Bunganic I, Belousova EA, et al. $3 \mathrm{~g}$ mesalazine granules are superior to $9 \mathrm{mg}$ budesonide for achieving remission in active ulcerative colitis: a double-blind, double-dummy, randomised trial. J Crohns Colitis 2011;5:129-38.

96 Van Assche G, Manguso F, Zibellini M, et al. Oral prolonged release beclomethasone dipropionate and prednisone in the treatment of active ulcerative colitis: results from a double-blind, randomized, parallel group study. Am J Gastroentero/ 2015;110:708-15.

97 Campieri M, Adamo S, Valpiani D, et al. Oral beclometasone dipropionate in the treatment of extensive and left-sided active ulcerative colitis: a multicentre randomised study. Aliment Pharmacol Ther 2003;17:1471-80.

98 Rizzello F, Gionchetti P, D'Arienzo A, et al. Oral beclometasone dipropionate in the treatment of active ulcerative colitis: a double-blind placebo-controlled study. Aliment Pharmacol Ther 2002;16:1109-16.

99 Ford AC, Bernstein CN, Khan KJ, et al. Glucocorticosteroid therapy in inflammatory bowel disease: systematic review and meta-analysis. Am J Gastroenterol 2011:106:590-9.

100 Baron JH, Connell AM, Kanaghinis TG, et al. Out-patient treatment of ulcerative colitis. Comparison between three doses of oral prednisone. Br Med J 1962:2:441-3.

101 Powell-Tuck J, Bown RL, Lennard-Jones JE. A comparison of oral prednisolone given as single or multiple daily doses for active proctocolitis. Scand J Gastroenterol 1978; 13:833-7.

102 Truelove SC, Witts LJ. Cortisone in ulcerative colitis; final report on a therapeutic trial. Br Med J 1955;2:1041-8.

103 Faubion WA, Loftus EV, Harmsen WS, et al. The natural history of corticosteroid therapy for inflammatory bowel disease: A population-based study. Gastroenterology 2001;121:255-60.

104 Roberts SE, Williams JG, Yeates D, et al. Mortality in patients with and without colectomy admitted to hospital for ulcerative colitis and Crohn's disease: record linkage studies. BMJ 2007;335:1033.

105 Randall J, Singh B, Warren BF, et al. Delayed surgery for acute severe colitis is associated with increased risk of postoperative complications. Br J Surg 2010:97:404-9.

106 Turner D, Walsh CM, Steinhart AH, et al. Response to corticosteroids in severe ulcerative colitis: a systematic review of the literature and a meta-regression. Clin Gastroenterol Hepatol 2007:5:103-10.

107 Rhodes JM, Robinson R, Beales I, et al. Clinical trial: oral prednisolone metasulfobenzoate (Predocol) vs. oral prednisolone for active ulcerative colitis. Aliment Pharmacol Ther 2008;27:228-40.

108 Wang Y, Parker CE, Feagan BG, et al. Oral 5-aminosalicylic acid for maintenance of remission in ulcerative colitis. Cochrane Database Syst Rev 2016:CD000544.

109 Marshall JK, Thabane M, Steinhart AH, et al. Rectal 5-aminosalicylic acid for maintenance of remission in ulcerative colitis. Cochrane Database Syst Rev 2012;11:CD004118.

110 Ford AC, Khan KJ, Sandborn WJ, et al. Efficacy of topical 5-aminosalicylates in preventing relapse of quiescent ulcerative colitis: a meta-analysis. Clin Gastroenterol Hepatol 2012;10:513-9.

111 Li W, Zhang Z-M, Jiang X-L. Once daily vs multiple daily mesalamine therapy for mild to moderate ulcerative colitis: a meta-analysis. Colorectal Dis 2016;18:0214-23.

112 Feagan BG, MacDonald JK. Once daily oral mesalamine compared to conventional dosing for induction and maintenance of remission in ulcerative colitis: a systematic review and meta-analysis. Inflamm Bowel Dis 2012;18:1785-94.

113 Tong JL, Huang ML, Xu XT, et al. Once-daily versus multiple-daily mesalamine for patients with ulcerative colitis: a meta-analysis. J Dig Dis 2012;13:200-7.

114 Ford AC, Khan KJ, Sandborn WJ, et al. Once-daily dosing vs. conventional dosing schedule of mesalamine and relapse of quiescent ulcerative colitis: systematic review and meta-analysis. Am J Gastroenterol 2011;106:2070-7.

115 Kane SV, Cohen RD, Aikens JE, et al. Prevalence of nonadherence with maintenance mesalamine in quiescent ulcerative colitis. Am J Gastroenterol 2001;96:2929-33.
116 Feagan BG, Chande N, MacDonald JK. Are there any differences in the efficacy and safety of different formulations of oral 5-ASA used for induction and maintenance of remission in ulcerative colitis? evidence from cochrane reviews. Inflamm Bowel Dis 2013;19:1-40.

117 Sandborn WJ. Rational selection of oral 5-aminosalicylate formulations and prodrugs for the treatment of ulcerative colitis. Am J Gastroenterol 2002;97:2939-41.

118 Khan KJ, Dubinsky MC, Ford AC, et al. Efficacy of immunosuppressive therapy for inflammatory bowel disease: a systematic review and meta-analysis. Am J Gastroentero/ 2011;106:630-42.

119 Gisbert JP, Linares PM, McNicholl AG, et al. Meta-analysis: the efficacy of azathioprine and mercaptopurine in ulcerative colitis. Aliment Pharmacol Ther 2009:30:126-37.

120 Timmer A, Patton PH, Chande N, et al. Azathioprine and 6-mercaptopurine for maintenance of remission in ulcerative colitis. Cochrane Database Syst Rev 2016:CD000478.

121 Rutgeerts P, Sandborn WJ, Feagan BG, et al. Infliximab for induction and maintenance therapy for ulcerative colitis. N Engl I Med Overseas Ed 2005; 353:2462-76.

122 Panaccione R, Ghosh S, Middleton S, et al. Combination therapy with infliximab and azathioprine is superior to monotherapy with either agent in ulcerative colitis. Gastroenterology 2014;146:392-400.

123 Armuzzi A, Pugliese D, Danese S, et al. Infliximab in steroid-dependent ulcerative colitis: effectiveness and predictors of clinical and endoscopic remission. Inflamm Bowel Dis 2013;19:1065-72.

124 Armuzzi A, Pugliese D, Danese $S$, et al. Long-term combination therapy with infliximab plus azathioprine predicts sustained steroid-free clinical benefit in steroid-dependent ulcerative colitis. Inflamm Bowel Dis 2014;20:1368-74.

125 Ferrante $M$, Vermeire $S$, Fidder $H$, et al. Long-term outcome after infliximab for refractory ulcerative colitis. J Crohns Colitis 2008;2:219-25.

126 Oussalah A, Evesque L, Laharie D, et al. A multicenter experience with infliximab for ulcerative colitis: outcomes and predictors of response, optimization, colectomy, and hospitalization. Am J Gastroenterol 2010;105:2617-25.

127 Reinisch W, Sandborn WJ, Hommes DW, et al. Adalimumab for induction of clinical remission in moderately to severely active ulcerative colitis: results of a randomised controlled trial. Gut 2011;60:780-7.

128 Sandborn WJ, van Assche G, Reinisch W, et al. Adalimumab induces and maintains clinical remission in patients with moderate-to-severe ulcerative colitis. Gastroenterology 2012;142:257-65.

129 Colombel J-F, Sandborn WJ, Ghosh S, et al. Four-year maintenance treatment with adalimumab in patients with moderately to severely active ulcerative colitis: Data from ULTRA 1, 2, and 3. Am J Gastroenterol 2014;109:1771-80

130 Sandborn WJ, Sakuraba A, Wang A, et al. Comparison of real-world outcomes of adalimumab and infliximab for patients with ulcerative colitis in the United States. Curr Med Res Opin 2016:32:1233-41.

131 Armuzzi A, Taxonera C, Panes J, et al. P365 Real-world effectiveness of adalimumab in patients with ulcerative colitis. J Crohns Colitis 2016;10(Suppl 1):S280-2.

132 Sandborn WJ, Feagan BG, Marano C, et al. Subcutaneous golimumab induces clinical response and remission in patients with moderate-to-severe ulcerative colitis. Gastroenterology 2014;146:85-95.

133 Samaan MA, Pavlidis P, Digby-Bell J, et al. Golimumab: early experience and medium-term outcomes from two UK tertiary IBD centres. Frontline Gastroenterol 2018;9:221-31

134 Thorlund K, Druyts E, Toor K, et al. Comparative efficacy of golimumab, infliximab, and adalimumab for moderately to severely active ulcerative colitis: a network meta-analysis accounting for differences in trial designs. Expert Rev Gastroenterol Hepatol 2015;9:693-700

135 Kristian T, Eric D, Kabirraaj T. P-026 Applying probabilistic rank ordering methodology to existing network meta-analysis of adalimumab, golimumab and infliximab for moderate-to-severe ulcerative colitis. Inflamm Bowel Dis 2014;20(Suppl 1):S32-4.

136 Kawalec PaweÅ, Pilc A. An indirect comparison of infliximab versus adalimumab or golimumab for active ulcerative colitis. Arch Med Sci 2016;5:1097-109.

137 Technology appraisal guidance [TA329] Infliximab, adalimumab and golimumab for treating moderately to severely active ulcerative colitis after the failure of conventional therapy. National Institute for Health and Care Excellence. 2015. https://www.nice.org.uk/guidance/ta329 (Accessed 12 Dec 2018).

138 Feagan BG, Rutgeerts P, Sands BE, et al. Vedolizumab as induction and maintenance therapy for ulcerative colitis. N Engl J Med Overseas Ed 2013;369:699-710.

139 Feagan BG, Rubin DT, Danese $S$, et al. Efficacy of vedolizumab induction and maintenance therapy in patients with ulcerative colitis, regardless of prior exposure to tumor necrosis factor antagonists. Clin Gastroenterol Hepatol 2017;15:229-39. 
140 Narula N, Peerani F, Meserve J, et al. Open: Vedolizumab for ulcerative colitis: treatment outcomes from the VICTORY consortium. Am J Gastroenterol 2018;113:1345-54.

141 Kopylov U, Verstockt B, Biedermann L, et al. Effectiveness and safety of vedolizumab in anti-TNF-naive patients with inflammatory bowel disease-a multicenter retrospective European study. Inflamm Bowel Dis 2018;24:2442-51.

142 Sands BE, Peyrin-Biroulet L, Loftus EV, et al. 416a - Vedolizumab shows superior efficacy versus adalimumab: results of Varsity — the first head-to-head study of biologic therapy for moderate-to-severe ulcerative colitis. Gastroenterology 2019;156:S-81.

143 Sandborn WJ, Ghosh S, Panes J, et al. Tofacitinib, an oral Janus kinase inhibitor, in active ulcerative colitis. N Eng/ J Med Overseas Ed 2012;367:616-24.

144 Sandborn WJ, Su C, Sands BE, et al. Tofacitinib as induction and maintenance therapy for ulcerative colitis. N Engl J Med Overseas Ed 2017;376:1723-36.

145 Panés J, Bressler B, Colombel J-F, et al. P516 Efficacy and safety of tofacitinib retreatment for ulcerative colitis after treatment interruption: Results from the OCTAVE clinical trials. J Crohn's Colitis 2018;12(Suppl 1):S366-7.

146 Sands BE, Moss AC, Armuzzi A, et al. DOP026 Efficacy and safety of dose escalation to tofacitinib $10 \mathrm{mg}$ BID for patients with ulcerative colitis following loss of response on tofacitinib $5 \mathrm{mg}$ BID maintenance therapy: results from OCTAVE open. J Crohns Colitis 2018;12(supplement_1):S049.

147 Bachelez H, van de Kerkhof PC, Strohal R, et al. Tofacitinib versus etanercept or placebo in moderate-to-severe chronic plaque psoriasis: a phase 3 randomised non-inferiority trial. Lancet 2015;386:552-61.

148 Cohen SB, Tanaka Y, Mariette X, et al. Long-term safety of tofacitinib for the treatment of rheumatoid arthritis up to 8.5 years: integrated analysis of data from the global clinical trials. Ann Rheum Dis 2017;76:1253-62.

149 ClinicalTrials.gov. Safety Study Of Tofacitinib Versus Tumor Necrosis Factor (TNF) Inhibitor In Subjects With Rheumatoid Arthritis. 2019. https://clinicaltrials.gov/ct2/ show/NCT02092467 [Accessed 19 May 2019].

150 XELJANZ (tofacitinib): Increased risk of pulmonary embolism and mortality in rheumatoid arthritis patients receiving $10 \mathrm{mg}$ twice daily in a clinical trial. Pfizer. 2019. https://assets.publishing.service.gov.uk/media/5cb5a218ed915d3f 4da780e6/Xeljanz-March-2019.pdf [Accessed 19 May 2019].

151 European Medicines Agency. Restrictions in use of Xeljanz while EMA reviews risk of blood clots in lungs. 2019. https://www.ema.europa.eu/en/news/ restrictions-use-xeljanz-while-ema-reviews-risk-blood-clots-lungs [Accessed 19 May 2019].

152 National Institute for Health and Care Excellence. Technology appraisal guidance [TA547] Tofacitinib for moderately to severely active ulcerative colitis. 2018. https:// www.nice.org.uk/guidance/ta547 [Accessed 14 Dec 2018].

153 Sands BE, Sandborn WJ, Panaccione R, et al. LB01 Safety and efficacy of ustekinumab induction therapy in patients with moderate to severe ulcerative colitis: results from the Phase 3 UNIFI study. United Eur Gastroenterol J 2018:6:1586-97.

154 Sandborn WJ, Sands BE, Panaccione R, et al. OP37 Efficacy and safety of ustekinumab as maintenance therapy in ulcerative colitis: Week 44 results from UNIFI. J Crohns Colitis 2019;13(Suppl 1):S025-6.

155 Wang Y, MacDonald JK, Vandermeer B, et al. Methotrexate for maintenance of remission in ulcerative colitis. Cochrane Database Syst Rev 2015:CD007560

156 Carbonnel F, Colombel JF, Filippi J, et al. Methotrexate is not superior to placebo for inducing steroid-free remission, but induces steroid-free clinical remission in a larger proportion of patients with ulcerative colitis. Gastroenterology 2016;150:380-8.

157 Herfarth H, Barnes EL, Valentine JF, et al. Methotrexate is not superior to placebo in maintaining steroid-free response or remission in ulcerative colitis. Gastroenterology 2018;155:1098-108

158 Danese S, Fiorino G, Peyrin-Biroulet L, et al. Biological agents for moderately to severely active ulcerative colitis: a systematic review and network meta-analysis. Ann Intern Med 2014;160:704-11.

159 Vickers AD, Ainsworth C, Mody R, et al. Systematic review with network metaanalysis: comparative efficacy of biologics in the treatment of moderately to severely active ulcerative colitis. PLoS One 2016;11:e0165435.

160 Moćko P, Kawalec P, Pilc A. Safety profile of biologic drugs in the therapy of ulcerative colitis: a systematic review and network meta-analysis. Pharmacotherapy 2016;36:870-9.

161 Bonovas S, Lytras T, Nikolopoulos G, et al. Systematic review with network meta-analysis: comparative assessment of tofacitinib and biological therapies for moderate-to-severe ulcerative colitis. Aliment Pharmacol Ther 2018;47:454-65.

162 Rubin DT, Ashaye A, Zhang Y, et al. Efficacy of tofacitinib and biologics as induction and maintenance therapy for moderately-to-severely active ulcerative colitis: a systematic review and network meta-analysis: Presidential Poster Award. Am J Gastroentero/ 2018;113(Suppl):S402-3.
163 Vermeire S, Colombel J-F, Feagan BG, et al. OP26 Long-term safety of vedolizumab in ulcerative colitis and Crohn's disease: final results from the GEMINI LTS study. J Crohn's Colitis 2019;13(Suppl1):S018-20.

164 Amiot A, Grimaud J-C, Peyrin-Biroulet L, et al. Effectiveness and safety of vedolizumab induction therapy for patients with inflammatory bowel disease. Clin Gastroenterol Hepatol 2016;14:1593-601.

165 Colombel JF, Sands BE, Rutgeerts P, et al. The safety of vedolizumab for ulcerative colitis and Crohn's disease. Gut 2017;66:839-51.

166 Dubinsky MC, Peyrin-Biroulet L, Melmed GY, et al. Efficacy of tofacitinib in patients with ulcerative colitis by prior tumor necrosis factor inhibitor treatment status: Results from octave induction and maintenance studies. Am J Gastroenterol 2017;112(Suppl 1):S354.

167 Harris MS, Lichtenstein GR. Review article: delivery and efficacy of topical 5-aminosalicylic acid (mesalazine) therapy in the treatment of ulcerative colitis. Aliment Pharmacol Ther 2011;33:996-1009.

168 Safdi M, DeMicco M, Sninsky C, et al. A double-blind comparison of oral versus rectal mesalamine versus combination therapy in the treatment of distal ulcerative colitis. Am J Gastroenterol 1997;92:1867-71.

169 Farup PG, Hovde Ø, Halvorsen FA, et al. Mesalazine suppositories versus hydrocortisone foam in patients with distal ulcerative colitis. A comparison of the efficacy and practicality of two topical treatment regimens. Scand J Gastroenterol 1995:30:164-70.

170 Cohen RD, Woseth DM, Thisted RA, et al. A meta-analysis and overview of the literature on treatment options for left-sided ulcerative colitis and ulcerative proctitis. Am J Gastroenterol 2000;95:1263-76.

171 Marshall JK, Thabane M, Steinhart AH, et al. Rectal 5-aminosalicylic acid for induction of remission in ulcerative colitis. Cochrane Database Syst Rev 2010:CD004115.

172 Römkens TE, Kampschreur MT, Drenth JP, et al. High mucosal healing rates in 5-ASA-treated ulcerative colitis patients: results of a meta-analysis of clinical trials. Inflamm Bowel Dis 2012;18:2190-8.

173 Andus T, Kocjan A, Müser M, et al. Clinical trial: a novel high-dose $1 \mathrm{~g}$ mesalamine suppository (Salofalk) once daily is as efficacious as a 500-mg suppository thrice daily in active ulcerative proctitis. Inflamm Bowel Dis 2010;16:1947-56.

174 Lamet M. A multicenter, randomized study to evaluate the efficacy and safety of mesalamine suppositories $1 \mathrm{~g}$ at bedtime and $500 \mathrm{mg}$ twice daily in patients with active mild-to-moderate ulcerative proctitis. Dig Dis Sci 2011;56:513-22.

175 Hanauer S, Good LI, Goodman MW, et al. Long-term use of mesalamine (Rowasa) suppositories in remission maintenance of ulcerative proctitis. Am J Gastroentero 2000;95:1749-54

176 Cohen RD, Dalal SR. Systematic Review: Rectal therapies for the treatment of distal forms of ulcerative colitis. Inflamm Bowel Dis 2015;21:1719-36.

177 Kruis W, Neshta V, Pesegova M, et al. Budesonide suppositories are effective and safe for treating acute ulcerative proctitis. Clin Gastroenterol Hepatol 2019:17:98-106.

178 Lamb CA, Lamb EIM, Mansfield JC, et al. Sexually transmitted infections manifesting as proctitis. Frontline Gastroenterol 2013;4:32-40.

179 Mallet A-L, Bouguen G, Conroy G, et al. Azathioprine for refractory ulcerative proctitis: A retrospective multicenter study. Dig Liver Dis 2017;49:280-5.

180 Kent A, Keshav S. Managing intractable proctitis and the problematic pouch. Dig Dis 2014;32:427-37.

181 Meier J, Sturm A. Current treatment of ulcerative colitis. World J Gastroenterol 2011;17:3204-12.

182 Wu XR, Liu XL, Katz S, et al. Pathogenesis, diagnosis, and management of ulcerative proctitis, chronic radiation proctopathy, and diversion proctitis. Inflamm Bowel Dis 2015;21:703-15.

183 Bouguen $\mathrm{G}$, Roblin X, Bourreille $A$, et al. Infliximab for refractory ulcerative proctitis. Aliment Pharmacol Ther 2010;31:1178-85.

184 Lawrance IC, Baird A, Lightower D, et al. Efficacy of rectal tacrolimus for induction therapy in patients with resistant ulcerative proctitis. Clin Gastroenterol Hepatol 2017; 15:1248-55.

185 Hawthorne AB, Logan RF, Hawkey CJ, et al. Randomised controlled trial of azathioprine withdrawal in ulcerative colitis. BMJ 1992:305:20-2.

186 Travis SP, Tysk C, de Silva HJ, et al. Optimum dose of olsalazine for maintaining remission in ulcerative colitis. Gut 1994;35:1282-6.

187 Fockens P, Mulder CJJ, Tytgat GNJ, et al. Comparison of the efficacy and safety of 1.5 compared with $3.0 \mathrm{~g}$ oral slow-release mesalazine (Pentasa) in the maintenance treatment of ulcerative colitis. Eur J Gastroenterol Hepatol 1995;7:1025-30.

188 Ardizzone S, Petrillo M, Imbesi V, et al. Is maintenance therapy always necessary for patients with ulcerative colitis in remission? Aliment Pharmacol Ther 1999;13:373-9.

189 Bernstein CN, Eaden J, Steinhart AH, et al. Cancer prevention in inflammatory bowel disease and the chemoprophylactic potential of 5-aminosalicylic acid. Inflamm Bowel Dis 2002;8:356-61. 
190 Flores BM, O'Connor A, Moss AC. Impact of mucosal inflammation on risk of colorectal neoplasia in patients with ulcerative colitis: a systematic review and meta-analysis. Gastrointest Endosc 2017;86:1006-11.

191 Andersen NN, Jess T. Has the risk of colorectal cancer in inflammatory bowel disease decreased? World J Gastroenterol 2013;19:7561-8.

192 Torres J, Boyapati RK, Kennedy NA, et al. Systematic review of effects of withdrawal of immunomodulators or biologic agents from patients with inflammatory bowel disease. Gastroenterology 2015;149:1716-30.

193 Pratico' C, Capozzi N, Rizzello F, et al. P370 Prospective withdrawal trial of azathioprine (AZA) in ulcerative colitis (UC). J Crohns Colitis 2014;8(Suppl 1):S219-20.

194 Kennedy NA, Kalla R, Warner B, et al. Thiopurine withdrawal during sustained clinical remission in inflammatory bowel disease: relapse and recapture rates, with predictive factors in 237 patients. Aliment Pharmacol Ther 2014;40:1313-23.

195 Cassinotti A, Actis GC, Duca P, et al. Maintenance treatment with azathioprine in ulcerative colitis: outcome and predictive factors after drug withdrawal. Am J Gastroenterol 2009;104:2760-7.

196 Seah D, De Cruz P. Review article: the practical management of acute severe ulcerative colitis. Aliment Pharmacol Ther 2016;43:482-513.

197 Turner D, Otley AR, Mack D, et al. Development, validation, and evaluation of a pediatric ulcerative colitis activity index: a prospective multicenter study. Gastroenterology 2007;133:423-32.

198 Osei-Bimpong A, Meek JH, Lewis SM. ESR or CRP? A comparison of their clinical utility. Hematology 2007;12:353-7.

199 Dinesen LC, Walsh AJ, Protic MN, et al. The pattern and outcome of acute severe colitis. J Crohns Colitis 2010;4:431-7.

200 Williams JG, Alam MF, Alrubaiy L, et al. Infliximab versus ciclosporin for steroidresistant acute severe ulcerative colitis (CONSTRUCT): a mixed methods, openlabel, pragmatic randomised trial. Lancet Gastroenterol Hepatol 2016;1:15-24.

201 Aratari A, Papi C, Clemente V, et al. Colectomy rate in acute severe ulcerative colitis in the infliximab era. Dig Liver Dis 2008;40:821-6.

202 Lynch RW, Churchhouse AM, Protheroe A, et al. Predicting outcome in acute severe ulcerative colitis: comparison of the Travis and Ho scores using UK IBD audit data. Aliment Pharmacol Ther 2016;43:1132-41.

203 Rosenberg W, Ireland A, Jewell DP. High-dose methylprednisolone in the treatment of active ulcerative colitis. J Clin Gastroentero/ 1990;12:40-1.

204 Chen JH, Andrews JM, Kariyawasam V, et al. Review article: acute severe ulcerative colitis - evidence-based consensus statements. Aliment Pharmacol Ther 2016:44:127-44

205 Islam MS, Grainger SL. Do we know how to use corticosteroids in acute severe ulcerative colitis? Frontline Gastroenterol 2012;3:248-51.

206 Wiles A, Bredin F, Chukualim B, et al. In the treatment of flares of inflammatory bowel disease, intravenous hydrocortisone causes greater falls in blood potassium and more severe episodes of hypokalaemia than methylprednisolone. Gut 2011;60(Suppl 1):A223-4

207 Bossa F, Fiorella S, Caruso N, et al. Continuous infusion versus bolus administration of steroids in severe attacks of ulcerative colitis: a randomized, double-blind trial. Am J Gastroenterol 2007;102:601-8.

208 Choshen S, Finnamore H, Auth MK, et al. Corticosteroid dosing in pediatric acute severe ulcerative colitis: a propensity score analysis. J Pediatr Gastroenterol Nutr 2016;63:58-64.

209 Truelove SC, Jewell DP. Intensive intravenous regimen for severe attacks of ulcerative colitis. Lancet 1974;1:1067-70.

210 Hart AL, Ng SC. Review article: the optimal medical management of acute severe ulcerative colitis. Aliment Pharmacol Ther 2010;32:615-27.

211 Grainge MJ, West J, Card TR. Venous thromboembolism during active disease and remission in inflammatory bowel disease: a cohort study. Lancet 2010;375:657-63.

212 Nguyen GC, Sam J. Rising prevalence of venous thromboembolism and its impact on mortality among hospitalized inflammatory bowel disease patients. Am J Gastroenterol 2008;103:2272-80

213 Shen J, Ran ZH, Tong JL, et al. Meta-analysis: The utility and safety of heparin in the treatment of active ulcerative colitis. Aliment Pharmacol Ther 2007;26:653-63.

214 Issa M, Vijayapal A, Graham MB, et al. Impact of Clostridium difficile on inflammatory bowel disease. Clin Gastroenterol Hepatol 2007;5:345-51.

215 Nguyen GC, Kaplan GG, Harris ML, et al. A national survey of the prevalence and impact of Clostridium difficile infection among hospitalized inflammatory bowel disease patients. Am J Gastroenterol 2008;103:1443-50.

216 Murthy SK, Steinhart AH, Tinmouth J, et al. Impact of Clostridium difficile colitis on 5 -year health outcomes in patients with ulcerative colitis. Aliment Pharmacol Ther 2012;36:1032-9.

217 Navaneethan U, Mukewar S, Venkatesh PG, et al. Clostridium difficile infection is associated with worse long term outcome in patients with ulcerative colitis. J Crohns Colitis 2012;6:330-6.
218 Negrón ME, Barkema HW, Rioux K, et al. Clostridium difficile infection worsens the prognosis of ulcerative colitis. Can J Gastroenterol Hepatol 2014; $28: 373-80$.

219 Horton HA, Dezfoli S, Berel D, et al. Antibiotics for treatment of Clostridium difficile infection in hospitalized patients with inflammatory bowel disease. Antimicrob Agents Chemother 2014;58:5054-9.

220 Lees CW, Heys D, Ho GT, et al. A retrospective analysis of the efficacy and safety of infliximab as rescue therapy in acute severe ulcerative colitis. Aliment Pharmacol Ther 2007;26:411-9.

221 Kaur M, Berel D, Vasiliauskas EA, et al. Sa1916 A combination of serum albumin and band neutrophil count is predictive of short-term colectomy following infliximab treatment for severe steroid refractory ulcerative colitis. Gastroenterology 2012;142(Suppl 1):S358

222 Mokhele NN, Thomson SR, Watermeyer GA. Predictors of emergency colectomy in patients admitted with acute severe ulcerative colitis. S Afr J Surg 2017;55:20-6.

223 Gibson DJ, Hartery K, Doherty J, et al. CRP/Albumin Ratio: An early predictor of steroid responsiveness in acute severe ulcerative colitis. J Clin Gastroenterol 2018;52:e48-e52.

224 Carbonnel F, Lavergne A, Lémann M, et al. Colonoscopy of acute colitis. A safe and reliable tool for assessment of severity. Dig Dis Sci 1994;39:1550-7.

225 Daperno M, Sostegni R, Scaglione N, et al. Outcome of a conservative approach in severe ulcerative colitis. Dig Liver Dis 2004;36:21-8.

226 Cacheux W, Seksik P, Lemann M, et al. Predictive factors of response to cyclosporine in steroid-refractory ulcerative colitis. Am J Gastroenterol 2008;103:637-42.

227 Monterubbianesi R, Aratari A, Armuzzi A, et al. Infliximab three-dose induction regimen in severe corticosteroid-refractory ulcerative colitis: early and late outcome and predictors of colectomy. J Crohns Colitis 2014;8:852-8

228 Corte C, Fernandopulle N, Catuneanu AM, et al. Association between the ulcerative colitis endoscopic index of severity (UCEIS) and outcomes in acute severe ulcerative colitis. J Crohns Colitis 2015;9:376-81.

229 Fernandes SR, Santos P, Miguel Moura C, et al. The use of a segmental endoscopic score may improve the prediction of clinical outcomes in acute severe ulcerative colitis. Rev Esp Enferm Dig 2016;108:697-702.

230 Lichtiger S, Present DH, Kornbluth A, et al. Cyclosporine in severe ulcerative colitis refractory to steroid therapy. N Engl J Med 1994:330:1841-5.

231 Van Assche G, D'Haens G, Noman M, et al. Randomized, double-blind comparison of $4 \mathrm{mg} / \mathrm{kg}$ versus $2 \mathrm{mg} / \mathrm{kg}$ intravenous cyclosporine in severe ulcerative colitis. Gastroenterology 2003;125:1025-31.

232 D'Haens G, Lemmens L, Geboes K, et al. Intravenous cyclosporine versus intravenous corticosteroids as single therapy for severe attacks of ulcerative colitis. Gastroenterology 2001;120:1323-9.

233 Moskovitz DN, Van Assche G, Maenhout B, et al. Incidence of colectomy during long-term follow-up after cyclosporine-induced remission of severe ulcerative colitis. Clin Gastroenterol Hepatol 2006:4:760-5.

234 Ordás I, Domènech $\mathrm{E}$, Mañosa $\mathrm{M}$, et al. Long-term efficacy and safety of cyclosporine in a cohort of steroid-refractory acute severe ulcerative colitis patients from the ENEIDA Registry (1989-2013): a nationwide multicenter study. Am J Gastroenterol 2017;112:1709-18.

235 Cohen RD, Stein R, Hanauer SB. Intravenous cyclosporin in ulcerative colitis: a fiveyear experience. Am J Gastroenterol 1999;94:1587-92.

236 Cheifetz AS, Stern J, Garud S, et al. Cyclosporine is safe and effective in patients with severe ulcerative colitis. J Clin Gastroentero/ 2011:45:107-12.

237 Campbell S, Travis S, Jewell D. Ciclosporin use in acute ulcerative colitis. Eur J Gastroenterol Hepatol 2005;17:79-84.

238 Sternthal MB, Murphy SJ, George J, et al. Adverse events associated with the use of cyclosporine in patients with inflammatory bowel disease. Am J Gastroenterol 2008;103:937-43.

239 Järnerot G, Hertervig E, Friis-Liby I, et al. Infliximab as rescue therapy in severe to moderately severe ulcerative colitis: a randomized, placebo-controlled study. Gastroenterology 2005;128:1805-11.

240 Sjöberg M, Magnuson A, Björk J, et al. Infliximab as rescue therapy in hospitalised patients with steroid-refractory acute ulcerative colitis: a long-term follow-up of 211 Swedish patients. Aliment Pharmacol Ther 2013;38:377-87.

241 Gustavsson A, Järnerot G, Hertervig E, et al. Clinical trial: colectomy after rescue therapy in ulcerative colitis - 3-year follow-up of the Swedish-Danish controlled infliximab study. Aliment Pharmacol Ther 2010;32:984-9.

242 Hayes MJ, Stein AC, Sakuraba A. Comparison of efficacy, pharmacokinetics, and immunogenicity between infliximab mono- versus combination therapy in ulcerative colitis. J Gastroenterol Hepatol 2014;29:1177-85.

243 Laharie D, Bourreille A, Branche J, et al. Ciclosporin versus infliximab in patients with severe ulcerative colitis refractory to intravenous steroids: a parallel, openlabel randomised controlled trial. Lancet 2012;380:1909-15. 
244 Laharie D, Bourreille A, Branche J, et al. Long-term outcome of patients with steroid-refractory acute severe UC treated with ciclosporin or infliximab. Gut 2018;67:237-43.

245 Narula N, Marshall JK, Colombel JF, et al. Systematic review and meta-analysis: infliximab or cyclosporine as rescue therapy in patients with severe ulcerative colitis refractory to steroids. Am J Gastroenterol 2016;111:477-91.

246 Chaparro M, Burgueño P, Iglesias E, et al. Infliximab salvage therapy after failure of ciclosporin in corticosteroid-refractory ulcerative colitis: a multicentre study. Aliment Pharmacol Ther 2012;35:275-83.

247 Maser EA, Deconda D, Lichtiger S, et al. Cyclosporine and infliximab as rescue therapy for each other in patients with steroid-refractory ulcerative colitis. Clin Gastroenterol Hepatol 2008;6:1112-6.

248 Narula N, Fine M, Colombel JF, et al. Systematic review: Sequential rescue therapy in severe ulcerative colitis: do the benefits outweigh the risks? Inflamm Bowel Dis 2015;21:1683-94.

249 Seow CH, Newman A, Irwin SP, et al. Trough serum infliximab: a predictive factor of clinical outcome for infliximab treatment in acute ulcerative colitis. Gut 2010;59:49-54

250 Adedokun OJ, Sandborn WJ, Feagan BG, et al. Association between serum concentration of infliximab and efficacy in adult patients with ulcerative colitis. Gastroenterology 2014;147:1296-307

251 Hindryckx P, Novak G, Vande Casteele N, et al. Review article: dose optimisation of infliximab for acute severe ulcerative colitis. Aliment Pharmacol Ther 2017; 45:617-30.

252 Yarur AJ, Jain A, Sussman DA, et al. The association of tissue anti-TNF drug levels with serological and endoscopic disease activity in inflammatory bowel disease: the ATLAS study. Gut 2016;65:249-55.

253 Brandse JF, Mathôt RA, van der Kleij D, et al. Pharmacokinetic features and presence of antidrug antibodies associated with response to infliximab induction therapy in patients with moderate to severe ulcerative colitis. Clin Gastroenterol Hepatol 2016;14:251-8

254 Gibson DJ, Heetun ZS, Redmond CE, et al. An accelerated infliximab induction regimen reduces the need for early colectomy in patients with acute severe ulcerative colitis. Clin Gastroenterol Hepatol 2015;13:330-5.

255 Choy MC, Seah D, Faleck DM, et al. Systematic review and meta-analysis: optimal salvage therapy in acute severe ulcerative colitis. Inflamm Bowel Dis 2019;25:1169-86.

256 Rice-Oxley JM, Truelove S. Ulcerative colitis course and prognosis. The Lancet 1950;255:663-6.

257 Billioud V, Ford AC, Tedesco ED, et al. Preoperative use of anti-TNF therapy and postoperative complications in inflammatory bowel diseases: a meta-analysis. J Crohns Colitis 2013;7:853-67.

258 Abelson JS, Michelassi F, Mao J, et al. Higher surgical morbidity for ulcerative colitis patients in the era of biologics. Ann Surg 2018;268:311-7.

259 Hyman NH, Cataldo P, Osler T. Urgent subtotal colectomy for severe inflammatory bowel disease. Dis Colon Rectum 2005;48:70-3.

260 Powar MP, Martin P, Croft AR, et al. Surgical outcomes in steroid refractory acute severe ulcerative colitis: the impact of rescue therapy. Colorectal Dis 2013;15:374-9.

261 Coakley BA, Telem D, Nguyen S, et al. Prolonged preoperative hospitalization correlates with worse outcomes after colectomy for acute fulminant ulcerative colitis. Surgery 2013;153:242-8

262 Kaplan GG, McCarthy EP, Ayanian JZ, et al. Impact of hospital volume on postoperative morbidity and mortality following a colectomy for ulcerative colitis. Gastroenterology 2008;134:680-7.

263 Fornaro R, Caratto M, Barbruni G, et al. Surgical and medical treatment in patients with acute severe ulcerative colitis. J Dig Dis 2015;16:558-67.

264 Kornbluth A, Sachar DB. Ulcerative colitis practice guidelines in adults: American College Of Gastroenterology, Practice Parameters Committee. Am J Gastroenterol 2010;105:501-23.

265 Bitton A, Buie D, Enns R, et al. Treatment of hospitalized adult patients with severe ulcerative colitis: Toronto consensus statements. Am J Gastroenterol 2012;107:179-94

266 Alves A, Panis Y, Bouhnik Y, et al. Subtotal colectomy for severe acute colitis: a 20-year experience of a tertiary care center with an aggressive and early surgical policy. J Am Coll Surg 2003;197:379-85.

267 Holubar SD, Larson DW, Dozois EJ, et al. Minimally invasive subtotal colectomy and ileal pouch-anal anastomosis for fulminant ulcerative colitis: a reasonable approach? Dis Colon Rectum 2009;52:187-92.

268 Buchs NC, Bloemendaal ALA, Wood CPJ, et al. Subtotal colectomy for ulcerative colitis: lessons learned from a tertiary centre. Colorectal Dis 2017; 19:0153-61.

269 Øresland T, Bemelman WA, Sampietro GM, et al. European evidence based consensus on surgery for ulcerative colitis. J Crohns Colitis 2015;9:4-25.
270 Nordenvall C, Myrelid P, Ekbom A, et al. Probability, rate and timing of reconstructive surgery following colectomy for inflammatory bowel disease in Sweden: a population-based cohort study. Colorectal Dis 2015;17:882-90.

271 Murphy PB, Khot Z, Vogt KN, et al. Quality of life after total proctocolectomy with ileostomy or IPAA: a systematic review. Dis Colon Rectum 2015;58:899-908

272 Koerdt S, Jehle EC, Kreis ME, et al. Quality of life after proctocolectomy and ileal pouch-anal anastomosis in patients with ulcerative colitis. Int J Colorectal Dis 2014;29:545-54.

273 Fazio VW, Kiran RP, Remzi FH, et al. Ileal pouch anal anastomosis: analysis of outcome and quality of life in 3707 patients. Ann Surg 2013:257:679-85.

274 Heikens JT, de Vries J, van Laarhoven CJ. Quality of life, health-related quality of life and health status in patients having restorative proctocolectomy with ileal pouch-anal anastomosis for ulcerative colitis: a systematic review. Colorectal Dis 2012;14:536-44.

275 Tulchinsky H, Dotan I, Halpern Z, et al. A longitudinal study of quality of life and functional outcome of patients with ulcerative colitis after proctocolectomy with ileal pouch-anal anastomosis. Dis Colon Rectum 2010;53:866-73.

276 Dunker MS, Bemelman WA, Slors JF, et al. Functional outcome, quality of life, body image, and cosmesis in patients after laparoscopic-assisted and conventional restorative proctocolectomy: a comparative study. Dis Colon Rectum 2001:44:1800-7.

277 Burns EM, Bottle A, Aylin P, et al. Volume analysis of outcome following restorative proctocolectomy. Br J Surg 2011;98:408-17.

278 Tekkis PP, Fazio VW, Lavery IC, et al. Evaluation of the learning curve in ileal pouchanal anastomosis surgery. Ann Surg 2005;241:262-8.

279 Raval MJ, Schnitzler M, O'Connor Bl, et al. Improved outcome due to increased experience and individualized management of leaks after ileal pouch-anal anastomosis. Ann Surg 2007;246:763-70

280 Mathis KL, Benavente-Chenhalls LA, Dozois EJ, et al. Short- and long-term surgical outcomes in patients undergoing proctocolectomy with ileal pouch-anal anastomosis in the setting of primary sclerosing cholangitis. Dis Colon Rectum 2011;54:787-92.

281 Pavlides M, Cleland J, Rahman M, et al. Outcomes after ileal pouch anal anastomosis in patients with primary sclerosing cholangitis. J Crohns Colitis 2014;8:662-70

282 Rahman M, Desmond P, Mortensen N, et al. The clinical impact of primary sclerosing cholangitis in patients with an ileal pouch-anal anastomosis for ulcerative colitis. Int J Colorectal Dis 2011:26:553-9.

283 Wasmuth $\mathrm{HH}$, Tranø G, Endreseth $\mathrm{BH}$, et al. Primary sclerosing cholangitis and extraintestinal manifestations in patients with ulcerative colitis and ileal pouch-ana anastomosis. J Gastrointest Surg 2010;14:1099-104.

284 Trivedi PJ, Reece J, Laing RW, et al. The impact of ileal pouch-anal anastomosis on graft survival following liver transplantation for primary sclerosing cholangitis. Aliment Pharmacol Ther 2018:48:322-32.

285 Tavernier N, Fumery M, Peyrin-Biroulet L, et al. Systematic review: fertility in non-surgically treated inflammatory bowel disease. Aliment Pharmacol Ther 2013;38:847-53.

286 Rajaratnam SG, Eglinton TW, Hider P, et al. Impact of ileal pouch-anal anastomosis on female fertility: meta-analysis and systematic review. Int J Colorectal Dis 2011;26:1365-74

287 Waljee A, Waljee J, Morris AM, et al. Threefold increased risk of infertility: a metaanalysis of infertility after ileal pouch anal anastomosis in ulcerative colitis. Gut 2006:55:1575-80.

288 Tulchinsky H, Averboukh F, Horowitz N, et al. Restorative proctocolectomy impairs fertility and pregnancy outcomes in women with ulcerative colitis. Colorectal Dis 2013;15:842-7

289 Mortier PE, Gambiez L, Karoui M, et al. Colectomy with ileorectal anastomosis preserves female fertility in ulcerative colitis. Gastroentero/ Clin Biol 2006:30:594-7.

290 Indar AA, Efron JE, Young-Fadok TM. Laparoscopic ileal pouch-anal anastomosis reduces abdominal and pelvic adhesions. Surg Endosc 2009;23:174-7.

291 Bartels SA, D'Hoore A, Cuesta MA, et al. Significantly increased pregnancy rates after laparoscopic restorative proctocolectomy: a cross-sectional study. Ann Surg 2012;256:1045-8.

292 Beyer-Berjot L, Maggiori L, Birnbaum D, et al. A total laparoscopic approach reduces the infertility rate after ileal pouch-anal anastomosis: a 2-center study. Ann Surg 2013;258:275-82

293 van der Ploeg VA, Maeda Y, Faiz OD, et al. The prevalence of chronic peri-pouch sepsis in patients treated for antibiotic-dependent or refractory primary idiopathic pouchitis. Colorectal Dis 2017:19:827-31.

294 McLaughlin SD, Clark SK, Tekkis PP, et al. Review article: restorative proctocolectomy, indications, management of complications and follow-up-a guide for gastroenterologists. Aliment Pharmacol Ther 2008;27:895-909. 
295 Kistangari G, Lopez R, Shen B. Frequency and risk factors of Clostridium difficile infection in hospitalized patients with pouchitis: a population-based study. Inflamm Bowel Dis 2017;23:661-71.

296 Navaneethan U, Shen B. Diagnosis and management of pouchitis and ileoanal pouch dysfunction. Curr Gastroenterol Rep 2010;12:485-94.

297 Li Y, Wu B, Shen B. Diagnosis and differential diagnosis of Crohn's disease of the ileal pouch. Curr Gastroenterol Rep 2012;14:406-13.

298 Sandborn WJ. Pouchitis following ileal pouch-anal anastomosis: definition, pathogenesis, and treatment. Gastroenterology 1994;107:1856-60.

299 Shen B, Achkar JP, Connor JT, et al. Modified pouchitis disease activity index: a simplified approach to the diagnosis of pouchitis. Dis Colon Rectum 2003;46:748-53.

300 McLaughlin SD, Clark SK, Thomas-Gibson S, et al. Guide to endoscopy of the ileo-anal pouch following restorative proctocolectomy with ileal pouch-anal anastomosis; indications, technique, and management of common findings. Inflamm Bowel Dis 2009:15:1256-63.

301 Segal JP, Ding NS, Worley G, et al. Systematic review with meta-analysis: the management of chronic refractory pouchitis with an evidence-based treatment algorithm. Aliment Pharmacol Ther 2017;45:581-92.

302 Hurst RD, Molinari M, Chung TP, et al. Prospective study of the incidence, timing and treatment of pouchitis in 104 consecutive patients after restorative proctocolectomy. Arch Surg 1996;131:497-500.

303 Shen B, Achkar JP, Lashner BA, et al. A randomized clinical trial of ciprofloxacin and metronidazole to treat acute pouchitis. Inflamm Bowel Dis 2001;7:301-5.

304 Singh S, Stroud AM, Holubar SD, et al. Treatment and prevention of pouchitis after ileal pouch-anal anastomosis for chronic ulcerative colitis. Cochrane Database Syst Rev 2015;11:CD001176.

305 Gionchetti P, Rizzello F, Helwig U, et al. Prophylaxis of pouchitis onset with probiotic therapy: a double-blind, placebo-controlled trial. Gastroenterology 2003;124:1202-9.

306 Scaioli E, Sartini A, Liverani E, et al. Sulfasalazine in prevention of pouchitis after proctocolectomy with ileal pouch-anal anastomosis for ulcerative colitis. Dig Dis Sci 2017:62:1016-24.

307 Gionchetti P, Rizzello F, Venturi A, et al. Oral bacteriotherapy as maintenance treatment in patients with chronic pouchitis: a double-blind, placebo-controlled trial. Gastroenterology 2000;119:305-9.

308 Mimura T, Rizzello F, Helwig U, et al. Once daily high dose probiotic therapy (VSL\#3) for maintaining remission in recurrent or refractory pouchitis. Gut 2004;53:108-14.

309 Pronio A, Montesani C, Butteroni C, et al. Probiotic administration in patients with ileal pouch-anal anastomosis for ulcerative colitis is associated with expansion of mucosal regulatory cells. Inflamm Bowel Dis 2008;14:662-8.

310 Kuisma J, Mentula S, Jarvinen $\mathrm{H}$, et al. Effect of Lactobacillus rhamnosus GG on ileal pouch inflammation and microbial flora. Aliment Pharmacol Ther 2003;17:509-15.

311 Shen J, Zuo ZX, Mao AP. Effect of probiotics on inducing remission and maintaining therapy in ulcerative colitis, Crohn's disease, and pouchitis: meta-analysis of randomized controlled trials. Inflamm Bowel Dis 2014;20:21-35.

312 Kuisma J, Järvinen $\mathrm{H}$, Kahri $\mathrm{A}$, et al. Factors associated with disease activity of pouchitis after surgery for ulcerative colitis. Scand I Gastroenterol 2004;39:544-8.

313 Penna C, Dozois R, Tremaine W, et al. Pouchitis after ileal pouch-anal anastomosis for ulcerative colitis occurs with increased frequency in patients with associated primary sclerosing cholangitis. Gut 1996;38:234-9.

314 Machiels K, Sabino J, Vandermosten L, et al. Specific members of the predominant gut microbiota predict pouchitis following colectomy and IPAA in UC. Gut 2017:66:79-88.

315 Landy J, Walker AW, Li JV, et al. Variable alterations of the microbiota, without metabolic or immunological change, following faecal microbiota transplantation in patients with chronic pouchitis. Sci Rep 2015;5:12955.

316 Mullish BH, Quraishi MN, Segal JP, et al. The use of faecal microbiota transplant as treatment for recurrent or refractory Clostridium difficile infection and other potential indications: joint British Society of Gastroenterology (BSG) and Healthcare Infection Society (HIS) guidelines. Gut 2018;67:1920-41.

317 Gionchetti P, Rizzello F, Poggioli G, et al. Oral budesonide in the treatment of chronic refractory pouchitis. Aliment Pharmacol Ther 2007;25:1231-6.

318 Gionchetti P, Calabrese C, Calafiore A, et al. Oral beclomethasone dipropionate in chronic refractory pouchitis. J Crohns Colitis 2014;8:649-53.

319 McLaughlin SD, Clark SK, Tekkis PP, et al. The bacterial pathogenesis and treatment of pouchitis. Therap Adv Gastroenterol 2010;3:335-48.

320 Samaan MA, de Jong D, Sahami S, et al. Incidence and severity of prepouch ileitis: a distinct disease entity or a manifestation of refractory pouchitis? Inflamm Bowel Dis 2016;22:662-8

321 Kjær MD, Qvist N, Nordgaard-Lassen I, et al. Adalimumab in the treatment of chronic pouchitis. A randomized double-blind, placebo-controlled trial. Scand J Gastroenterol 2019;54:AB269.
322 Bär F, Kühbacher T, Dietrich NA, et al. Vedolizumab in the treatment of chronic, antibiotic-dependent or refractory pouchitis. Aliment Pharmacol Ther 2018;47:581-7.

323 Uchino M, Ikeuchi H, Matsuoka H, et al. Topical tacrolimus therapy for antibioticrefractory pouchitis. Dis Colon Rectum 2013:56:1166-73.

324 Tekkis PP, Lovegrove RE, Tilney HS, et al. Long-term failure and function after restorative proctocolectomy - a multi-centre study of patients from the UK National Ileal Pouch Registry. Colorectal Dis 2010;12:433-41.

325 Tulchinsky H, Dotan I, Alper A, et al. Comprehensive pouch clinic concept for follow-up of patients after ileal pouch anal anastomosis: report of 3 years' experience in a tertiary referral center. Inflamm Bowel Dis 2008;14:1125-32.

326 lleoanal Pouch Report. Association of Coloprotoctology of Great Britain and Ireland, https://www.acpgbi.org.uk/content/uploads/2016/07/leoanal-PouchReport-2017-FINAL.compressed.pdf [Accessed 14 Dec 2018].

327 Selvaggi F, Pellino G, Canonico S, et al. Systematic review of cuff and pouch cancer in patients with ileal pelvic pouch for ulcerative colitis. Inflamm Bowel Dis 2014;20:1296-308.

328 Block M, Börjesson L, Willén R, et al. Neoplasia in the colorectal specimens of patients with ulcerative colitis and ileal pouch-anal anastomosis - need for routine surveillance? Scand I Gastroenterol 2015;50:528-35.

329 Scarpa M, van Koperen PJ, Ubbink DT, et al. Systematic review of dysplasia after restorative proctocolectomy for ulcerative colitis. Br I Surg 2007:94:534-45.

330 Kariv R, Remzi FH, Lian L, et al. Preoperative colorectal neoplasia increases risk for pouch neoplasia in patients with restorative proctocolectomy. Gastroenterology 2010;139:806-12

331 Das P, Johnson MW, Tekkis PP, et al. Risk of dysplasia and adenocarcinoma following restorative proctocolectomy for ulcerative colitis. Colorectal Dis 2007:9:15-27.

332 Kuiper T, Vlug MS, van den Broek FJ, et al. The prevalence of dysplasia in the ileoanal pouch following restorative proctocolectomy for ulcerative colitis with associated dysplasia. Colorectal Dis 2012;14:469-73.

333 Wu XR, Remzi FH, Liu XL, et al. Disease course and management strategy of pouch neoplasia in patients with underlying inflammatory bowel diseases. Inflamm Bowel Dis 2014;20:2073-82.

334 Zhu H, Wu XR, Queener E, et al. Clinical value of surveillance pouchoscopy in asymptomatic ileal pouch patients with underlying inflammatory bowel disease. Surg Endosc 2013;27:4325-32.

335 Börjesson L, Willén R, Haboubi N, et al. The risk of dysplasia and cancer in the ileal pouch mucosa after restorative proctocolectomy for ulcerative proctocolitis is low: a long-term term follow-up study. Colorectal Dis 2004;6:494-8.

336 Lennard-Jones JE. Classification of inflammatory bowel disease. Scand J Gastroenterol Supp/ 1989;170:2-6.

337 Peyrin-Biroulet L, Billioud V, D'Haens G, et al. Development of the Paris definition of early Crohn's disease for disease-modification trials: results of an international expert opinion process. Am J Gastroenterol 2012;107:1770-6.

338 Hyams JS. Standardized recording of parameters related to the natural history of inflammatory bowel disease: from Montreal to Paris. Dig Dis 2014;32:337-44.

339 Ashton JJ, Bonduelle Q, Mossotto E, et al. Endoscopic and histological assessment of paediatric inflammatory bowel disease over a 3-year follow-up period. J Pediatr Gastroenterol Nutr 2018:66:402-9.

340 Ashton JJ, Coelho T, Ennis S, et al. Endoscopic versus histological disease extent at presentation of paediatric inflammatory bowel disease. J Pediatr Gastroenterol Nutr 2016;62:246-51.

341 Fernandes MA, Verstraete SG, Garnett EA, et al. Addition of histology to the Paris classification of pediatric Crohn disease alters classification of disease location. J Pediatr Gastroenterol Nutr 2016:62:242-5.

342 van Hees $\mathrm{PA}$, van Elteren $\mathrm{PH}$, van Lier $\mathrm{HJ}$, et al. An index of inflammatory activity in patients with Crohn's disease. Gut 1980:21:279-86.

343 Sandborn WJ, Feagan BG, Hanauer SB, et al. A review of activity indices and efficacy endpoints for clinical trials of medical therapy in adults with Crohn's disease. Gastroenterology 2002;122:512-30.

344 European Medicines Agency. Guideline on the development of new medicinal products for the treatment of Crohn's Disease. June $2018 \mathrm{https}: / / \mathrm{www}$. ema.europa.eu/documents/scientific-guideline/guideline-development-newmedicinal-products-treatment-crohns-disease-revision-2_en.pdf (Accessed 14 Dec 2018).

345 Harvey RF, Bradshaw JM. A simple index of Crohn's-disease activity. Lancet 1980;1:514

346 Kim AH, Roberts C, Feagan BG, et al. Developing a standard set of patient-centred outcomes for inflammatory bowel disease: an international, cross-disciplinary consensus. J Crohns Colitis 2018;12:408-18.

347 Bodger K, Ormerod C, Shackcloth D, et al. Development and validation of a rapid, generic measure of disease control from the patient's perspective: the IBD-contro questionnaire. Gut 2014;63:1092-102. 
348 Gracie DJ, Williams CJ, Sood R, et al. Poor correlation between clinical disease activity and mucosal inflammation, and the role of psychological comorbidity in inflammatory bowel disease. Am J Gastroenterol 2016;111:541-51.

349 Pariente B, Cosnes J, Danese S, et al. Development of the Crohn's disease digestive damage score, the Lémann score. Inflamm Bowel Dis 2011;17:1415-22.

350 Allen PB, Olivera P, Emery P, et al. Review article: moving towards common therapeutic goals in Crohn's disease and rheumatoid arthritis. Aliment Pharmacol Ther 2017;45:1058-72.

351 Khanna R, Levesque BG, Sandborn WJ. IBD: Measuring what counts--endoscopic assessment in IBD. Nat Rev Gastroenterol Hepatol 2014;11:9-10.

352 Khanna R, Bouguen G, Feagan BG, et al. A systematic review of measurement of endoscopic disease activity and mucosal healing in Crohn's disease: recommendations for clinical trial design. Inflamm Bowel Dis 2014;20:1850-61.

353 Mary JY, Modigliani R. Development and validation of an endoscopic index of the severity for Crohn's disease: a prospective multicentre study. Groupe d'Etudes Thérapeutiques des Affections Inflammatoires du Tube Digestif (GETAID). Gut 1989;30:983-9.

354 Daperno M, D'Haens G, Van Assche G, et al. Development and validation of a new, simplified endoscopic activity score for Crohn's disease: the SES-CD. Gastrointest Endosc 2004:60:505-12.

355 Khanna R, Zou G, Stitt L, et al. Responsiveness of endoscopic indices of disease activity for Crohn's disease. Am J Gastroenterol 2017:112:1584-92.

356 Buchner AM, Lichtenstein GR. Editorial: Endoscopic scoring systems in Crohn's disease for evaluation of responsiveness to treatment: are we ready for the prime time of endoscopic assessment? Am J Gastroenterol 2017;112:1593-5.

357 Ordás I, Rimola J, Rodríguez S, et al. Accuracy of magnetic resonance enterography in assessing response to therapy and mucosal healing in patients with Crohn's disease. Gastroenterology 2014;146:374-82.

358 Steward MJ, Punwani S, Proctor I, et al. Non-perforating small bowel Crohn's disease assessed by MRI enterography: derivation and histopathological validation of an MR-based activity index. Eur J Radio/ 2012;81:2080-8.

359 Rutgeerts P, Geboes K, Vantrappen G, et al. Predictability of the postoperative course of Crohn's disease. Gastroenterology 1990;99:956-63.

360 Colombel JF, Panaccione R, Bossuyt P, et al. Effect of tight control management on Crohn's disease (CALM): a multicentre, randomised, controlled phase 3 trial. Lancet 2018:390:2779-89

361 Panaccione R, Colombel F, Bossuyt P, et al. OP017 Treat to target for Crohn's disease with adalimumab treatment is cost effective over 48 weeks: An economic assessment of the CALM trial. United Eur Gastroenterol J 2017:5(Suppl):A8-9.

362 Samuel S, Bruining DH, Loftus EV, et al. Endoscopic skipping of the distal terminal ileum in Crohn's disease can lead to negative results from ileocolonoscopy. Clin Gastroenterol Hepatol 2012;10:1253-9.

363 Marshall JK, Cawdron R, Zealley I, et al. Prospective comparison of small bowel meal with pneumocolon versus ileo-colonoscopy for the diagnosis of ileal Crohn's disease. Am J Gastroenterol 2004:99:1321-9.

364 Marshall JK, Hewak J, Farrow R, et al. Terminal ileal imaging with ileoscopy versus small-bowel meal with pneumocolon. J Clin Gastroenterol 1998;27:217-22.

365 Geboes K, Ectors N, D'Haens G, et al. Is ileoscopy with biopsy worthwhile in patients presenting with symptoms of inflammatory bowel disease? Am J Gastroenterol 1998:93:201-6.

366 Witte AM, Veenendaal RA, Van Hogezand RA, et al. Crohn's disease of the upper gastrointestinal tract: the value of endoscopic examination. Scand I Gastroenterol Supp/ 1998;225:100-5.

367 Annunziata ML, Caviglia R, Papparella LG, et al. Upper gastrointestinal involvement of Crohn's disease: a prospective study on the role of upper endoscopy in the diagnostic work-up. Dig Dis Sci 2012;57:1618-23.

368 Rutgeerts $P$, Onette E, Vantrappen $\mathrm{G}$, et al. Crohn's disease of the stomach and duodenum: A clinical study with emphasis on the value of endoscopy and endoscopic biopsies. Endoscopy 1980;12:288-94.

369 Lin J, McKenna BJ, Appelman HD. Morphologic findings in upper gastrointestinal biopsies of patients with ulcerative colitis: a controlled study. Am I Surg Pathol 2010:34:1-7.

370 Choi M, Lim S, Choi MG, et al. Effectiveness of capsule endoscopy compared with other diagnostic modalities in patients with small bowel Crohn's disease: a metaanalysis. Gut Liver 2017;11:62-72.

371 Taylor SA, Avni F, Cronin CG, et al. The first joint ESGAR/ ESPR consensus statement on the technical performance of cross-sectional small bowel and colonic imaging. Eur Radiol 2017;27:2570-82

372 Masselli G, Casciani E, Polettini E, et al. Comparison of MR enteroclysis with MR enterography and conventional enteroclysis in patients with Crohn's disease. Eur Radio/ 2008:18:438-47.

373 Horsthuis K, Bipat S, Bennink RJ, et al. Inflammatory bowel disease diagnosed with US, MR, scintigraphy, and CT: meta-analysis of prospective studies. Radiology 2008;247:64-79
374 Dong J, Wang H, Zhao J, et al. Ultrasound as a diagnostic tool in detecting active Crohn's disease: a meta-analysis of prospective studies. Eur Radiol 2014;24:26-33.

375 Liu W, Liu J, Xiao W, et al. A diagnostic accuracy meta-analysis of CT and MRI for the evaluation of small bowel Crohn disease. Acad Radiol 2017;24:1216-25.

376 Panés J, Bouzas R, Chaparro M, et al. Systematic review: the use of ultrasonography, computed tomography and magnetic resonance imaging for the diagnosis, assessment of activity and abdominal complications of Crohn's disease. Aliment Pharmacol Ther 2011:34:125-45.

377 Greenup AJ, Bressler B, Rosenfeld G. Medical imaging in small bowel Crohn's disease-computer tomography enterography, magnetic resonance enterography, and ultrasound: "Which One Is the Best for What?". Inflamm Bowel Dis 2016:22:1246-61.

378 Taylor SA, Mallett S, Bhatnagar G, et al. Diagnostic accuracy of magnetic resonance enterography and small bowel ultrasound for the extent and activity of newly diagnosed and relapsed Crohn's disease (METRIC): a multicentre trial. Lancet Gastroenterol Hepatol 2018;3:548-58.

379 Horsthuis K, Bipat S, Stokkers PC, et al. Magnetic resonance imaging for evaluation of disease activity in Crohn's disease: a systematic review. Eur Radiol 2009;19:1450-60.

380 Puylaert CA, Tielbeek JA, Bipat S, et al. Grading of Crohn's disease activity using CT, MRI, US and scintigraphy: a meta-analysis. Eur Radio/ 2015;25:3295-313.

381 Rimola J, Ordás I, Rodriguez S, et al. Magnetic resonance imaging for evaluation of Crohn's disease: validation of parameters of severity and quantitative index of activity. Inflamm Bowel Dis 2011;17:1759-68.

382 Serafin Z, Białecki M, Białecka A, et al. Contrast-enhanced ultrasound for detection of Crohn's disease activity: systematic review and meta-analysis. J Crohns Colitis 2016:10:354-62.

383 Rieder F, Latella G, Magro F, et al. European Crohn's and Colitis Organisation topical review on prediction, diagnosis and management of fibrostenosing Crohn's disease. J Crohns Colitis 2016;10:873-85.

384 Dillman JR, Swanson SD, Johnson LA, et al. Comparison of noncontrast MRI magnetization transfer and $\mathrm{T} 2$-weighted signal intensity ratios for detection of bowel wall fibrosis in a Crohn's disease animal model. J Magn Reson Imaging 2015:42:801-10.

385 Rimola J, Planell N, Rodríguez S, et al. Characterization of inflammation and fibrosis in Crohn's disease lesions by magnetic resonance imaging. Am J Gastroenterol 2015;110:432-40.

$386 \mathrm{Ma} \mathrm{X}$, Li Y, Jia H, et al. Contrast-enhanced ultrasound in the diagnosis of patients suspected of having active Crohn's disease: meta-analysis. Ultrasound Med Biol 2015:41:659-68.

387 Dillman JR, Stidham RW, Higgins PD, et al. US elastography-derived shear wave velocity helps distinguish acutely inflamed from fibrotic bowel in a Crohn disease animal model. Radiology 2013;267:757-66.

388 Jess T, Gamborg M, Matzen P, et al. Increased risk of intestinal cancer in Crohn's disease: a meta-analysis of population-based cohort studies. Am J Gastroenterol 2005:100:2724-9.

389 Laukoetter MG, Mennigen R, Hannig CM, et al. Intestinal cancer risk in Crohn's disease: a meta-analysis. J Gastrointest Surg 2011;15:576-83.

390 Fumery M, Pineton de Chambrun G, Stefanescu C, et al. Detection of dysplasia or cancer in $3.5 \%$ of patients with inflammatory bowel disease and colonic strictures. Clin Gastroenterol Hepatol 2015:13:1770-5.

391 Zakeri N, Pollok RC. Diagnostic imaging and radiation exposure in inflammatory bowel disease. World I Gastroenterol 2016:22:2165-78.

392 Desmond AN, O'Regan K, Curran C, et al. Crohn's disease: factors associated with exposure to high levels of diagnostic radiation. Gut 2008:57:1524-9.

393 Bousorra H, Labidi A, Fekih M, et al. Radiation exposure in Crohn's disease patients. Tunis Med 2016:94:385-9.

394 Pearce MS, Salotti JA, Little MP, et al. Radiation exposure from CT scans in childhood and subsequent risk of leukaemia and brain tumours: a retrospective cohort study. Lancet 2012;380:499-505.

395 Peloquin JM, Pardi DS, Sandborn WJ, et al. Diagnostic ionizing radiation exposure in a population-based cohort of patients with inflammatory bowel disease. Am J Gastroenterol 2008;103:2015-22.

396 Gandhi NS, Baker ME, Goenka AH, et al. Diagnostic accuracy of CT enterography for active inflammatory terminal ileal Crohn disease: comparison of full-dose and half-dose images reconstructed with FBP and half-dose images with SAFIRE. Radiology 2016;280:436-45.

397 Camera L, Liccardo I, Romano F, et al. Diagnostic efficacy of single-pass abdomina multidetector-row CT: prospective evaluation of a low dose protocol. Br J Radiol 2017:90:20160612.

398 Bourreille A, Ignjatovic A, Aabakken L, et al. Role of small-bowel endoscopy in the management of patients with inflammatory bowel disease: an international OMED ECCO consensus. Endoscopy 2009;41:618-37. 
399 Hookey L, Louw J, Wiepjes M, et al. Lack of benefit of active preparation compared with a clear fluid-only diet in small-bowel visualization for video capsule endoscopy: results of a randomized, blinded, controlled trial. Gastrointest Endosc 2017:85:187-93.

400 Yung DE, Rondonotti E, Sykes C, et al. Systematic review and meta-analysis: is bowel preparation still necessary in small bowel capsule endoscopy? Expert Rev Gastroenterol Hepatol 2017;11:979-93.

401 Rondonotti E, Spada C, Adler S, et al. Small-bowel capsule endoscopy and device-assisted enteroscopy for diagnosis and treatment of small-bowel disorders: European Society of Gastrointestinal Endoscopy (ESGE) Technical Review. Endoscopy 2018;50:423-46.

402 Dionisio PM, Gurudu SR, Leighton JA, et al. Capsule endoscopy has a significantly higher diagnostic yield in patients with suspected and established small-bowel Crohn's disease: a meta-analysis. Am J Gastroenterol 2010;105:1240-8.

403 Doherty GA, Moss AC, Cheifetz AS. Capsule endoscopy in suspected Crohn's disease: "yield" does not equal "diagnosis". Am I Gastroenterol 2010;105:2111.

404 Solem CA, Loftus EV, Fletcher JG, et al. Small-bowel imaging in Crohn's disease: a prospective, blinded, 4-way comparison trial. Gastrointest Endosc 2008;68:255-66.

405 Pennazio M, Spada C, Eliakim R, et al. Small-bowel capsule endoscopy and device-assisted enteroscopy for diagnosis and treatment of small-bowel disorders: European Society of Gastrointestinal Endoscopy (ESGE) Clinical Guideline. Endoscopy 2015;47:352-86.

406 Rezapour M, Amadi C, Gerson LB. Retention associated with video capsule endoscopy: systematic review and meta-analysis. Gastrointest Endosc 2017:85:1157-68

407 Enns RA, Hookey L, Armstrong D, et al. Clinical practice guidelines for the use of video capsule endoscopy. Gastroenterology 2017;152:497-514.

408 Xin L, Liao Z, Jiang YP, et al. Indications, detectability, positive findings, tota enteroscopy, and complications of diagnostic double-balloon endoscopy: a systematic review of data over the first decade of use. Gastrointest Endosc 2011;74:563-70.

409 Takenaka K, Ohtsuka K, Kitazume Y, et al. Comparison of magnetic resonance and balloon enteroscopic examination of the small intestine in patients with Crohn's disease. Gastroenterology 2014;147:334-42.

410 Arulanandan A, Dulai PS, Singh S, et al. Systematic review: Safety of balloon assisted enteroscopy in Crohn's disease. World I Gastroenterol 2016;22:8999-9011.

411 Despott EJ, Gupta A, Burling D, et al. Effective dilation of small-bowel strictures by double-balloon enteroscopy in patients with symptomatic Crohn's disease (with video). Gastrointest Endosc 2009;70:1030-6.

412 Bar-Meir S, Chowers Y, Lavy A, et al. Budesonide versus prednisone in the treatment of active Crohn's disease. The Israeli Budesonide Study Group. Gastroenterology 1998;115:835-40.

413 Moja L, Danese S, Fiorino G, et al. Systematic review with network meta-analysis: comparative efficacy and safety of budesonide and mesalazine (mesalamine) for Crohn's disease. Aliment Pharmacol Ther 2015;41:1055-65.

414 Rezaie A, Kuenzig ME, Benchimol El, et al. Budesonide for induction of remission in Crohn's disease. Cochrane Database Syst Rev 2015(6):CD000296.

415 Coward S, Kuenzig ME, Hazlewood G, et al. Comparative effectiveness of mesalamine, sulfasalazine, corticosteroids, and budesonide for the induction of remission in Crohn's disease: a Bayesian network meta-analysis. Inflamm Bowel Dis 2017:23:461-72

416 Dignass A, Stoynov S, Dorofeyev AE, et al. Once versus three times daily dosing of oral budesonide for active Crohn's disease: a double-blind, double-dummy, randomised trial. J Crohns Colitis 2014;8:970-80.

417 Benchimol El, Seow CH, Steinhart AH, et al. Traditional corticosteroids for induction of remission in Crohn's disease. Cochrane Database Syst Rev 2008:CD006792.

418 Summers RW, Switz DM, Sessions JT, et al. National Cooperative Crohn's Disease Study: results of drug treatment. Gastroenterology 1979;77:847-69.

419 Malchow H, Ewe K, Brandes JW, et al. European Cooperative Crohn's Disease Study (ECCDS): results of drug treatment. Gastroenterology 1984;86:249-66.

420 Dziechciarz P, Horvath A, Shamir R, et al. Meta-analysis: enteral nutrition in active Crohn's disease in children. Aliment Pharmacol Ther 2007:26:795-806.

421 Narula N, Dhillon A, Zhang D, et al. Enteral nutritional therapy for induction of remission in Crohn's disease. Cochrane Database Syst Rev 2018;4:CD000542.

422 Rigaud D, Cosnes J, Le Quintrec Y, et al. Controlled trial comparing two types of enteral nutrition in treatment of active Crohn's disease: elemental versus polymeric diet. Gut 1991;32:1492-7.

423 Royall D, Jeejeebhoy KN, Baker JP, et al. Comparison of amino acid v peptide based enteral diets in active Crohn's disease: clinical and nutritional outcome. Gut 1994;35:783-7

424 Lee J, Allen R, Ashley S, et al. British Dietetic Association evidence-based guidelines for the dietary management of Crohn's disease in adults. J Hum Nutr Diet 2014;27:207-18.
425 Wall CL, Gearry RB, Day AS. Treatment of active crohn's disease with exclusive and partial enteral nutrition: a pilot study in adults. Inflamm Intest Dis 2018;2:219-27.

426 Yang Q, Gao X, Chen $\mathrm{H}$, et al. Efficacy of exclusive enteral nutrition in complicated Crohn's disease. Scand I Gastroenterol 2017;52:995-1001.

427 Gatti S, Galeazzi T, Franceschini E, et al. Effects of the exclusive enteral nutrition on the microbiota profile of patients with Crohn's disease: a systematic review. Nutrients 2017:9:832.

428 Sigall-Boneh R, Levine A, Lomer M, et al. Research gaps in diet and nutrition in inflammatory bowel disease. A Topical Review by D-ECCO Working Group [Dietitians of ECCO]. J Crohns Colitis 2017;11:1407-19.

429 Lomer MC, Gourgey R, Whelan K. Current practice in relation to nutritional assessment and dietary management of enteral nutrition in adults with Crohn's disease. J Hum Nutr Diet 2014;27(Suppl 2):28-35.

430 Yamamoto T, Nakahigashi M, Saniabadi AR. Review article: diet and inflammatory bowel disease--epidemiology and treatment. Aliment Pharmacol Ther 2009;30:99-112.

431 Beattie RM, Schiffrin EJ, Donnet-Hughes A, et al. Polymeric nutrition as the primary therapy in children with small bowel Crohn's disease. Aliment Pharmacol Ther 1994;8:609-15

432 Fell JM, Paintin M, Arnaud-Battandier F, et al. Mucosal healing and a fall in mucosal pro-inflammatory cytokine mRNA induced by a specific oral polymeric diet in paediatric Crohn's disease. Aliment Pharmacol Ther 2000;14:281-9.

433 Borrelli O, Cordischi L, Cirulli M, et al. Polymeric diet alone versus corticosteroids in the treatment of active pediatric Crohn's disease: a randomized controlled openlabel trial. Clin Gastroenterol Hepatol 2006;4:744-53.

434 Wall CL, Day AS, Gearry RB. Use of exclusive enteral nutrition in adults with Crohn's disease: a review. World I Gastroenterol 2013;19:7652-60.

435 Zachos M, Tondeur M, Griffiths AM. Enteral nutritional therapy for induction of remission in Crohn's disease. Cochrane Database Syst Rev 2007:CD000542.

436 Forbes A, Escher J, Hébuterne X, et al. ESPEN guideline: Clinical nutrition in inflammatory bowel disease. Clin Nutr 2017;36:321-47.

437 Woolner JT, Parker TJ, Kirby GA, et al. The development and evaluation of a diet for maintaining remission in Crohn's disease. J Hum Nutr Diet 1998;11:1-11.

438 Faiman A, Mutalib M, Moylan A, et al. Standard versus rapid food reintroduction after exclusive enteral nutritional therapy in paediatric Crohn's disease. Eur $\int$ Gastroenterol Hepatol 2014;26:276-81.

439 Svolos V, Hansen R, Nichols B, et al. Treatment of active Crohn's disease with an ordinary food-based diet that replicates exclusive enteral nutrition. Gastroenterology 2019;156:1354-67.

440 Khan KJ, Ullman TA, Ford AC, et al. Antibiotic therapy in inflammatory bowel disease: a systematic review and meta-analysis. Am J Gastroenterol 2011;106:661-73

441 Prantera C, Lochs H, Grimaldi M, et al. Rifaximin-extended intestinal release induces remission in patients with moderately active Crohn's disease. Gastroenterology 2012;142:473-81.

442 Scribano ML. Role of Rifaximin in inflammatory bowel disease treatment. Mini Rev Med Chem 2015;16:225-9.

443 Levine A, Kori M, Kierkus J, et al. Azithromycin and metronidazole versus metronidazole-based therapy for the induction of remission in mild to moderate paediatric Crohn's disease : a randomised controlled trial. Gut 2019;68:239-47.

444 Selby W, Pavli P, Crotty B, et al. Two-year combination antibiotic therapy with clarithromycin, rifabutin, and clofazimine for Crohn's disease. Gastroenterology 2007;132:2313-9.

445 Graham DY, Hardi R, Welton T, et al. LB06 - A phase III randomized, double blind, placebo-controlled, multicenter, parallel group study to assess the efficacy and safety of fixed-dose combination RHB-104 in subjects with moderately to severely active Crohn's disease. United Eur Gastroenterol J 2018;6(Suppl).

446 Ponsioen CY, de Groof EJ, Eshuis EJ, et al. Laparoscopic ileocaecal resection versus infliximab for terminal ileitis in Crohn's disease: a randomised controlled, openabel, multicentre trial. Lancet Gastroenterol Hepatol 2017;2:785-92.

447 de Groof EJ, Stevens TW, Eshuis EJ, et al. Cost-effectiveness of laparoscopic ileocaecal resection versus infliximab treatment of terminal ileitis in Crohn's disease: the LIR!C Trial. Gut 2019. doi: 10.1136/gutjnl-2018-317539 [Epub ahead of print 11 Feb 2019].

448 Selinger CP, Parkes GC, Bassi A, et al. A multi-centre audit of excess steroid use in 1176 patients with inflammatory bowel disease. Aliment Pharmacol Ther 2017:46:964-73.

449 Beaugerie L, Seksik P, Nion-Larmurier I, et al. Predictors of Crohn's disease. Gastroenterology 2006;130:650-6.

450 Loly C, Belaiche J, Louis E. Predictors of severe Crohn's disease. Scand J Gastroenterol 2008:43:948-54.

451 Lee JC, Lyons PA, McKinney EF, et al. Gene expression profiling of CD8+ T cells predicts prognosis in patients with Crohn disease and ulcerative colitis. J Clin Invest 2011;121:4170-9 
452 Hanauer S, Sandborn WJ, Persson A, et al. Budesonide as maintenance treatment in Crohn's disease: a placebo-controlled trial. Aliment Pharmacol Ther 2005;21:363-71.

453 Sandborn WJ, Löfberg R, Feagan BG, et al. Budesonide for maintenance of remission in patients with Crohn's disease in medically induced remission: a predetermined pooled analysis of four randomized, double-blind, placebocontrolled trials. Am J Gastroenterol 2005;100:1780-7

454 Kuenzig ME, Rezaie A, Seow CH, et al. Budesonide for maintenance of remission in Crohn's disease. Cochrane Database Syst Rev 2014;8:CD002913.

455 Chande N, Patton PH, Tsoulis DJ, et al. Azathioprine or 6-mercaptopurine for maintenance of remission in Crohn's disease. Cochrane Database Syst Rev 2015;10:CD000067.

456 Hazlewood GS, Rezaie A, Borman M, et al. Comparative effectiveness of immunosuppressants and biologics for inducing and maintaining remission in Crohn's disease: a network meta-analysis. Gastroenterology 2015;148:344-54.

457 Feagan BG, Rochon J, Fedorak RN, et al. Methotrexate for the treatment of Crohn's disease. The North American Crohn's Study Group Investigators. N Engl J Med 1995;332:292-7.

458 McDonald JW, Wang Y, Tsoulis DJ, et al. Methotrexate for induction of remission in refractory Crohn's disease. Cochrane Database Syst Rev 2014;8:CD003459.

459 Feagan BG, Fedorak RN, Irvine EJ, et al. A comparison of methotrexate with placebo for the maintenance of remission in Crohn's disease. North American Crohn's Study Group Investigators. N Engl J Med 2000;342:1627-32.

460 Patel V, Wang Y, MacDonald JK, et al. Methotrexate for maintenance of remission in Crohn's disease. Cochrane Database Syst Rev 2014;8:CD006884.

461 Braun J, Kästner P, Flaxenberg P, et al. Comparison of the clinical efficacy and safety of subcutaneous versus oral administration of methotrexate in patients with active rheumatoid arthritis: results of a six-month, multicenter, randomized, double-blind, controlled, phase IV trial. Arthritis Rheum 2008;58:73-81.

462 Rozin A, Schapira D, Balbir-Gurman A, et al. Relapse of rheumatoid arthritis after substitution of oral for parenteral administration of methotrexate. Ann Rheum Dis 2002:61:756-7.

463 Kopylov U, Katsanos KH, van der Woude CJ, et al. European experience with methotrexate treatment in Crohn's disease: a multicenter retrospective analysis. Eur J Gastroenterol Hepatol 2016;28:802-6.

464 Turner D, Doveh E, Cohen A, et al. Efficacy of oral methotrexate in paediatric Crohn's disease: a multicentre propensity score study. Gut 2015;64:1898-904.

465 Kurnik D, Loebstein R, Fishbein E, et al. Bioavailability of oral vs. subcutaneous low-dose methotrexate in patients with Crohn's disease. Aliment Pharmacol Ther 2003; 18:57-63.

466 Wilson A, Patel V, Chande N, et al. Pharmacokinetic profiles for oral and subcutaneous methotrexate in patients with Crohn's disease. Aliment Pharmacol Ther 2013;37:340-5

467 Brooks PJ, Spruill WJ, Parish RC, et al. Pharmacokinetics of methotrexate administered by intramuscular and subcutaneous injections in patients with rheumatoid arthritis. Arthritis Rheum 1990;33:91-4.

468 Chhaya V, Saxena S, Cecil E, et al. Steroid dependency and trends in prescribing for inflammatory bowel disease - a 20-year national population-based study. Aliment Pharmacol Ther 2016;44:482-94

469 Schoepfer AM, Bortolotti M, Pittet V, et al. The gap between scientific evidence and clinical practice: 5-aminosalicylates are frequently used for the treatment of Crohn's disease. Aliment Pharmacol Ther 2014;40:930-7.

470 Akobeng AK, Zhang D, Gordon M, et al. Oral 5-aminosalicylic acid for maintenance of medically-induced remission in Crohn's disease. Cochrane Database Syst Rev 2016;9:CD003715.

471 Lim WC, Wang Y, MacDonald JK, et al. Aminosalicylates for induction of remission or response in Crohn's disease. Cochrane Database Syst Rev 2016;7:CD008870.

472 Lim WC, Hanauer S. Aminosalicylates for induction of remission or response in Crohn's disease. Cochrane Database Syst Rev 2010;12:CD008870.

473 Subramanian S, Ekbom A, Rhodes JM. Recent advances in clinical practice: a systematic review of isolated colonic Crohn's disease: the third IBD? Gut 2017;66:362-81

474 Hanauer SB, Feagan BG, Lichtenstein GR, et al. Maintenance infliximab for Crohn's disease: the ACCENT I randomised trial. Lancet 2002;359:1541-9.

475 Schnitzler F, Fidder H, Ferrante M, et al. Long-term outcome of treatment with infliximab in 614 patients with Crohn's disease: results from a single-centre cohort. Gut 2009;58:492-500.

476 Rutgeerts P, Feagan BG, Lichtenstein GR, et al. Comparison of scheduled and episodic treatment strategies of infliximab in Crohn's disease. Gastroenterology 2004;126:402-13.

477 Colombel JF, Sandborn WJ, Reinisch W, et al. Infliximab, azathioprine, or combination therapy for Crohn's disease. N Engl J Med 2010;362:1383-95.

478 Nuti F, Civitelli F, Bloise $S$, et al. Prospective evaluation of the achievement of mucosal healing with Anti-TNF- $\alpha$ therapy in a paediatric crohn's disease cohort. J Crohns Colitis 2016;10:5-12.
479 Hyams JS, Lerer T, Griffiths A, et al. Long-term outcome of maintenance infliximab therapy in children with Crohn's disease. Inflamm Bowel Dis 2009;15:816-22.

480 Sokol H, Seksik P, Carrat F, et al. Usefulness of co-treatment with immunomodulators in patients with inflammatory bowel disease treated with scheduled infliximab maintenance therapy. Gut 2010;59:1363-8.

481 Kennedy NA, Heap GA, Green HD, et al. Predictors of anti-TNF treatment failure in anti-TNF-naive patients with active luminal Crohn's disease: a prospective, multicentre, cohort study. Lancet Gastroenterol Hepatol 2019;4:341-53.

482 Feagan BG, McDonald JW, Panaccione R, et al. Methotrexate in combination with infliximab is no more effective than infliximab alone in patients with Crohn's disease. Gastroenterology 2014;146:681-8.

483 Schröder 0, Blumenstein I, Stein J. Combining infliximab with methotrexate for the induction and maintenance of remission in refractory Crohn's disease: a controlled pilot study. Eur J Gastroenterol Hepatol 2006;18:11-16.

484 Grossi V, Lerer T, Griffiths A, et al. Concomitant use of immunomodulators affects the durability of infliximab therapy in children with crohn's disease. Clin Gastroenterol Hepatol 2015;13:1748-56.

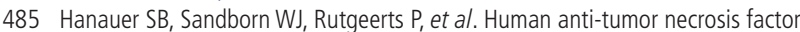
monoclonal antibody (adalimumab) in Crohn's disease: the CLASSIC-I trial. Gastroenterology 2006;130:323-33.

486 Colombel JF, Sandborn WJ, Rutgeerts P, et al. Adalimumab for maintenance of clinical response and remission in patients with Crohn's disease: the CHARM trial. Gastroenterology 2007;132:52-65.

487 Sandborn WJ, Rutgeerts $\mathrm{P}$, Enns R, et al. Adalimumab induction therapy for Crohn disease previously treated with infliximab: a randomized trial. Ann Intern Med 2007;146:829-38.

488 Rutgeerts P, Van Assche G, Sandborn WJ, et al. Adalimumab induces and maintains mucosal healing in patients with Crohn's disease: data from the EXTEND trial. Gastroenterology 2012;142:1102-11.

489 Colombel JF, Rutgeerts PJ, Sandborn WJ, et al. Adalimumab induces deep remission in patients with Crohn's disease. Clin Gastroenterol Hepatol 2014;12:414-22.

490 Kopylov U, Al-Taweel T, Yaghoobi M, et al. Adalimumab monotherapy versus combination therapy with immunomodulators in patients with Crohn's disease: a systematic review and meta-analysis. J Crohns Colitis 2014;8:1632-41.

491 Matsumoto T, Motoya S, Watanabe K, et al. Adalimumab monotherapy and a combination with azathioprine for Crohn's disease: a prospective, randomized trial. J Crohns Colitis 2016;10:1259-66.

492 Panaccione R, Sandborn WJ, D'Haens G, et al. Clinical benefit of long-term adalimumab treatment in patients with Crohn's disease following loss of response or intolerance to infliximab: 96-week efficacy data from GAIN/ADHERE Trials. J Crohns Colitis 2018;12:930-8.

493 Bond A, Dodd S, Fisher G, et al. Concurrent immunomodulator therapy is associated with higher adalimumab trough levels during scheduled maintenance therapy. Scand J Gastroenterol 2017;52:204-8.

494 Watanabe K, Matsumoto T, Hisamatsu T, et al. Clinical and pharmacokinetic factors associated with adalimumab-induced mucosal healing in patients with Crohn's disease. Clin Gastroenterol Hepatol 2018;16:542-9.

495 Sazonovs A, Kennedy NA, Bewshea C, et al. OTU-002 HLA-DQA1 contributes to the development of antibodies to anti-TNF therapy in Crohn's disease. Gut 2018;67(Suppl 1):A52.

496 Strik AS, van den Brink GR, Ponsioen C, et al. Suppression of anti-drug antibodies to infliximab or adalimumab with the addition of an immunomodulator in patients with inflammatory bowel disease. Aliment Pharmacol Ther 2017;45:1128-34.

497 Sandborn WJ, Feagan BG, Rutgeerts P, et al. Vedolizumab as induction and maintenance therapy for Crohn's disease. N Engl J Med 2013;369:711-21.

498 Sands BE, Sandborn WJ, Van Assche G, et al. Vedolizumab as induction and maintenance therapy for Crohn's disease in patients naive to or who have failed tumor necrosis factor antagonist therapy. Inflamm Bowel Dis 2017:23:97-106.

499 Wang MC, Zhang LY, Han W, et al. PRISMA--efficacy and safety of vedolizumab for inflammatory bowel diseases: a systematic review and meta-analysis of randomized controlled trials. Medicine 2014;93:e326.

500 Vermeire S, Loftus EV, Colombel JF, et al. Long-term efficacy of vedolizumab for Crohn's disease. J Crohns Colitis 2017:11:412-24.

501 Plevris N, Chuah C, Jenkinson P, et al. PWE-045 Vedolizumab results in reduced hospitalisation and steroid use over 1-year: Results from the Scottish Vedolizumab Cohort. Gut 2018;67(Suppl 1):A89.

502 Eriksson C, Marsal J, Bergemalm D, et al. Long-term effectiveness of vedolizumab in inflammatory bowel disease: a national study based on the Swedish National Quality Registry for Inflammatory Bowel Disease (SWIBREG). Scand I Gastroenterol 2017;52:722-9.

503 Faleck DM, Winters A, Chablaney S, et al. Shorter disease duration is associated with higher rates of response to vedolizumab in patients with Crohn's disease but not ulcerative colitis. Clin Gastroenterol Hepatol 2019. doi: 10.1016/j. cgh.2018.12.040. [Epub ahead of print 6 Jan 2019]. 
504 Ungaro RC, Colombel JF. Editorial: biologics in inflammatory bowel disease-time for direct comparisons. Aliment Pharmacol Ther 2017;46:68-9.

505 Bohm M, Sagi S, Fischer M, et al. Sa1723 - Comparative effectiveness of vedolizumab and tumor necrosis factor-antagonist therapy in Crohn's disease: a multicenter consortium propensity score-matched analysis. Gastroenterology 2018;154(6):S-369-70.

506 Feagan BG, Sandborn WJ, Gasink C, et al. Ustekinumab as induction and maintenance therapy for Crohn's disease. N Engl J Med 2016;375:1946-60.

507 Wils P, Bouhnik Y, Michetti P, et al. Subcutaneous ustekinumab provides clinical benefit for two-thirds of patients with Crohn's disease refractory to anti-tumor necrosis factor agents. Clin Gastroenterol Hepatol 2016;14:242-50.

508 Khorrami S, Ginard D, Marín-Jiménez I, et al. Ustekinumab for the Treatment of Refractory Crohn's Disease: The Spanish Experience in a Large Multicentre Openlabel Cohort. Inflamm Bowel Dis 2016;22:1662-9.

509 Kopylov U, Afif W, Cohen A, et al. Subcutaneous ustekinumab for the treatment of anti-TNF resistant Crohn's disease-the McGill experience. J Crohns Colitis 2014;8:1516-22.

510 Greenup AJ, Rosenfeld G, Bressler B. Ustekinumab use in Crohn's disease: a Canadian tertiary care centre experience. Scand J Gastroenterol 2017;52:1354-9.

511 Harris KA, Horst S, Gadani A, et al. Patients with refractory Crohn's disease successfully treated with ustekinumab. Inflamm Bowel Dis 2016;22:397-401.

512 Ma C, Fedorak RN, Kaplan GG, et al. Clinical, endoscopic and radiographic outcomes with ustekinumab in medically-refractory Crohn's disease: real world experience from a multicentre cohort. Aliment Pharmacol Ther 2017:45:1232-43.

513 Kawalec P, Moćko P. An indirect comparison of ustekinumab and vedolizumab in the therapy of TNF-failure Crohn's disease patients. J Comp Eff Res 2018;7:101-11.

514 Colombel JF, Reinisch W, Mantzaris GJ, et al. Randomised clinical trial: deep remission in biologic and immunomodulator naïve patients with Crohn's disease a SONIC post hoc analysis. Aliment Pharmacol Ther 2015;41:734-46.

515 Schreiber S, Reinisch W, Colombel JF, et al. Subgroup analysis of the placebocontrolled CHARM trial: increased remission rates through 3 years for adalimumabtreated patients with early Crohn's disease. J Crohns Colitis 2013;7:213-21.

516 Lichtenstein GR, Feagan BG, Cohen RD, et al. Serious infection and mortality in patients with Crohn's disease: more than 5 years of follow-up in the TREATTM registry. Am J Gastroenterol 2012;107:1409-22.

517 Hawkey CJ, Allez M, Clark MM, et al. Autologous hematopoetic stem cell transplantation for refractory Crohn disease: a randomized clinical trial. JAMA 2015;314:2524-34.

518 Snowden JA, Panés J, Alexander T, et al. Autologous haematopoietic stem cell transplantation (AHSCT) in severe Crohn's disease: a review on behalf of ECCO and EBMT. J Crohns Colitis 2018;12:476-88.

519 Sands BE, Katz S, Wolf DC, et al. A randomised, double-blind, sham-controlled study of granulocyte/monocyte apheresis for moderate to severe Crohn's disease. Gut 2013;62:1288-94.

520 Lazarev M, Huang C, Bitton A, et al. Relationship between proximal Crohn's disease location and disease behavior and surgery: a cross-sectional study of the IBD Genetics Consortium. Am J Gastroenterol 2013;108:106-12.

521 Flamant M, Trang C, Maillard O, et al. The prevalence and outcome of jejunal lesions visualized by small bowel capsule endoscopy in Crohn's disease. Inflamm Bowel Dis 2013;19:1390-6.

522 Keh C, Shatari T, Yamamoto T, et al. Jejunal Crohn's disease is associated with a higher postoperative recurrence rate than ileocaecal Crohn's disease. Colorectal Dis 2005; 7:366-8.

523 Mottet C, Vader JP, Felley C, et al. Appropriate management of special situations in Crohn's disease (upper gastro-intestinal; extra-intestinal manifestations; drug safety during pregnancy and breastfeeding): Results of a multidisciplinary international expert panel-EPACT II. J Crohns Colitis 2009;3:257-63.

524 Decker GA, Loftus EV, Pasha TM, et al. Crohn's disease of the esophagus: clinical features and outcomes. Inflamm Bowel Dis 2001;7:113-9.

525 Miest RY, Bruce AJ, Comfere NI, et al. A diagnostic approach to recurrent orofacial swelling: a retrospective study of 104 patients. Mayo Clin Proc 2017;92:1053-60.

526 Harty S, Fleming P, Rowland M, et al. A prospective study of the oral manifestations of Crohn's disease. Clin Gastroenterol Hepatol 2005;3:886-91.

527 White A, Nunes C, Escudier M, et al. Improvement in orofacial granulomatosis on a cinnamon- and benzoate-free diet. Inflamm Bowel Dis 2006;12:508-14.

528 Mutalib M, Bezanti K, Elawad M, et al. The role of exclusive enteral nutrition in the management of orofacial granulomatosis in children. World J Pediatr 2016;12:421-4

529 Fedele S, Fung PP, Bamashmous N, et al. Long-term effectiveness of intralesional triamcinolone acetonide therapy in orofacial granulomatosis: an observational cohort study. Br J Dermatol 2014;170:794-801.

530 Mentzer A, Goel R, Elliott T, et al. Azathioprine is effective for oral involvement in Crohn's disease but not for orofacial granulomatosis alone. J Oral Pathol Med 2016;45:312-8.
531 Elliott T, Campbell H, Escudier M, et al. Experience with anti-TNF- $\alpha$ therapy for orofacial granulomatosis. J Oral Pathol Med 2011;40:14-19.

532 Bouhnik Y, Carbonnel F, Laharie D, et al. Efficacy of adalimumab in patients with Crohn's disease and symptomatic small bowel stricture: a multicentre, prospective, observational cohort (CREOLE) study. Gut 2018;67:53-60.

533 Greco A, Caviglia GP, Brignolo P, et al. Glucose breath test and Crohn's disease: Diagnosis of small intestinal bacterial overgrowth and evaluation of therapeutic response. Scand J Gastroenterol 2015;50:1376-81.

534 Castiglione F, Rispo A, Di Girolamo E, et al. Antibiotic treatment of small bowel bacterial overgrowth in patients with Crohn's disease. Aliment Pharmacol Ther 2003:18:1107-12.

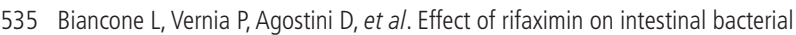
overgrowth in Crohn's disease as assessed by the H2-glucose breath Test. Current Med Res Opin 2000;16:14-20.

536 Frolkis AD, Dykeman J, Negrón ME, et al. Risk of surgery for inflammatory bowel diseases has decreased over time: a systematic review and meta-analysis of population-based studies. Gastroenterology 2013;145:996-1006.

537 Sokol H, Seksik P, Cosnes J. Complications and surgery in the inflammatory bowel diseases biological era. Curr Opin Gastroenterol 2014;30:378-84.

538 Rieder F, Zimmermann EM, Remzi FH, et al. Crohn's disease complicated by strictures: a systematic review. Gut 2013;62:1072-84.

539 Thienpont C, Van Assche G. Endoscopic and medical management of fibrostenotic Crohn's disease. Dig Dis 2014;32(Suppl 1):35-8.

540 Thienpont C, D'Hoore A, Vermeire S, et al. Long-term outcome of endoscopic dilatation in patients with Crohn's disease is not affected by disease activity or medical therapy. Gut 2010;59:320-4.

541 Navaneethan U, Lourdusamy V, Njei B, et al. Endoscopic balloon dilation in the management of strictures in Crohn's disease: a systematic review and metaanalysis of non-randomized trials. Surg Endosc 2016;30:5434-43.

542 Morar PS, Faiz O, Warusavitarne J, et al. Systematic review with meta-analysis: endoscopic balloon dilatation for Crohn's disease strictures. Aliment Pharmacol Ther 2015;42:1137-48.

543 Roy P, Kumar D. Strictureplasty. Br J Surg 2004;91:1428-37.

544 Fazio VW, Tjandra JJ, Lavery IC, et al. Long-term follow-up of strictureplasty in Crohn's disease. Dis Colon Rectum 1993:36:355-61.

545 Yamamoto T. Factors affecting recurrence after surgery for Crohn's disease. World I Gastroenterol 2005;11:3971-9.

546 Ozuner G, Fazio VW, Lavery IC, et al. Reoperative rates for Crohn's disease following strictureplasty. Long-term analysis. Dis Colon Rectum 1996;39:1199-203.

547 Fichera A, Lovadina S, Rubin M, et al. Patterns and operative treatment of recurrent Crohn's disease: a prospective longitudinal study. Surgery 2006;140:649-54.

548 Reese GE, Purkayastha S, Tilney HS, et al. Strictureplasty vs resection in small bowel Crohn's disease: an evaluation of short-term outcomes and recurrence. Colorectal Dis 2007:9:686-94.

549 Michelassi F, Taschieri A, Tonelli F, et al. An international, multicenter, prospective, observational study of the side-to-side isoperistaltic strictureplasty in Crohn's disease. Dis Colon Rectum 2007:50:277-84.

550 Campbell L, Ambe R, Weaver J, et al. Comparison of conventional and nonconventional strictureplasties in Crohn's disease: a systematic review and metaanalysis. Dis Colon Rectum 2012;55:714-26.

551 Roy P, Kumar D. Intervention-free interval following strictureplasty for Crohn's Disease. World I Surg 2006;30:1020-6.

552 Brooker JC, Beckett CG, Saunders BP, et al. Long-acting steroid injection after endoscopic dilation of anastomotic Crohn's strictures may improve the outcome: a retrospective case series. Endoscopy 2003;35:333-7.

553 East JE, Brooker JC, Rutter MD, et al. A pilot study of intrastricture steroid versus placebo injection after balloon dilatation of Crohn's strictures. Clin Gastroenterol Hepatol 2007:5:1065-9.

554 Atreja A, Aggarwal A, Dwivedi S, et al. Safety and efficacy of endoscopic dilation for primary and anastomotic Crohn's disease strictures. I Crohns Colitis 2014;8:392-400.

555 Pugmire BS, Gee MS, Kaplan JL, et al. Role of percutaneous abscess drainage in the management of young patients with Crohn disease. Pediatr Radiol 2016;46:653-9.

556 Garcia JC, Persky SE, Bonis PA, et al. Abscesses in Crohn's disease: outcome of medical versus surgical treatment. J Clin Gastroenterol 2001;32:409-12.

557 Gervais DA, Hahn PF, O'Neill MJ, et al. Percutaneous abscess drainage in Crohn disease: technical success and short- and long-term outcomes during 14 years. Radiology 2002;222:645-51.

558 Kaimakliotis $\mathrm{P}$, Simillis C, Harbord M, et al. A systematic review assessing medical treatment for rectovaginal and enterovesical fistulae in Crohn's disease. J Clin Gastroenterol 2016;50:714-21.

559 Zhang W, Zhu W, Li Y, et al. The respective role of medical and surgical therapy for enterovesical fistula in Crohn's disease. J Clin Gastroenterol 2014;48:708-11. 
560 de la Poza G, López-Sanroman A, Taxonera C, et al. Genital fistulas in female Crohn's disease patients.: clinical characteristics and response to therapy. J Crohns Colitis 2012:6:276-80.

561 Gionchetti P, Dignass A, Danese S, et al. 3rd European Evidence-based Consensus on the diagnosis and management of Crohn's disease 2016: Part 2: Surgical management and special situations. J Crohns Colitis 2017;11:135-49.

562 Ravindran P, Ansari N, Young CJ, et al. Definitive surgical closure of enterocutaneous fistula: outcome and factors predictive of increased postoperative morbidity. Colorectal Dis 2014;16:209-18.

563 Yaari S, Benson A, Aviran E, et al. Factors associated with surgery in patients with intra-abdominal fistulizing Crohn's disease. World J Gastroenterol 2016;22:10380-7

564 Gecse K, Khanna R, Stoker J, et al. Fistulizing Crohn's disease: Diagnosis and management. United Eur Gastroenterol J 2013;1:206-13.

565 Amiot A, Setakhr V, Seksik P, et al. Long-term outcome of enterocutaneous fistula in patients with Crohn's disease treated with anti-TNF therapy: a cohort study from the GETAID. Am J Gastroenterol 2014;109:1443-9.

566 Mawdsley JE, Hollington P, Bassett $\mathrm{P}$, et al. An analysis of predictive factors for healing and mortality in patients with enterocutaneous fistulas. Aliment Pharmacol Ther 2008:28:1111-21.

567 Sands BE, Anderson FH, Bernstein CN, et al. Infliximab maintenance therapy for fistulizing Crohn's disease. N Engl J Med 2004;350:876-85.

568 Orsoni P, Barthet M, Portier F, et al. Prospective comparison of endosonography, magnetic resonance imaging and surgical findings in anorectal fistula and abscess complicating Crohn's disease. Br J Surg 1999;86:360-4.

569 Schwartz DA, Wiersema MJ, Dudiak KM, et al. A comparison of endoscopic ultrasound, magnetic resonance imaging, and exam under anesthesia for evaluation of Crohn's perianal fistulas. Gastroenterology 2001;121:1064-72.

570 Buchanan GN, Halligan S, Bartram Cl, et al. Clinical examination, endosonography, and MR imaging in preoperative assessment of fistula in ano: comparison with outcome-based reference standard. Radiology 2004;233:674-81.

571 Garros A, Siproudhis L, Tchoundjeu B, et al. Magnetic resonance imaging and clinical assessments for perianal Crohn's disease: gain and limits. Dig Liver Dis 2014;46:1072-6.

572 Marzo M, Felice C, Pugliese D, et al. Management of perianal fistulas in Crohn's disease: an up-to-date review. World J Gastroentero/ 2015;21:1394-403.

573 Schwartz DA, Pemberton JH, Sandborn WJ. Diagnosis and treatment of perianal fistulas in Crohn disease. Ann Intern Med 2001;135:906-18.

574 Chidi VN, Schwartz DA. Imaging of perianal fistulizing Crohn's disease. Expert Rev Gastroenterol Hepatol 2015;9:797-806.

575 Regueiro M. The role of endoscopy in the evaluation of fistulizing Crohn's disease. Gastrointest Endosc Clin N Am 2002;12:621-33.

576 Bell SJ, Williams AB, Wiesel $P$, et al. The clinical course of fistulating Crohn's disease. Aliment Pharmacol Ther 2003;17:1145-51.

577 Regueiro M, Mardini H. Treatment of perianal fistulizing Crohn's disease with infliximab alone or as an adjunct to exam under anesthesia with seton placement. Inflamm Bowel Dis 2003;9:98-103.

578 Topstad DR, Panaccione R, Heine JA, et al. Combined seton placement, infliximab infusion, and maintenance immunosuppressives improve healing rate in fistulizing anorectal Crohn's disease: a single center experience. Dis Colon Rectum 2003;46:577-83.

579 Talbot C, Sagar PM, Johnston MJ, et al. Infliximab in the surgical management of complex fistulating anal Crohn's disease. Colorectal Dis 2005;7:164-8.

580 Yassin NA, Askari A, Warusavitarne J, et al. Systematic review: the combined surgical and medical treatment of fistulising perianal Crohn's disease. Aliment Pharmacol Ther 2014;40:741-9.

581 Hyder SA, Travis SP, Jewell DP, et al. Fistulating anal Crohn's disease: results of combined surgical and infliximab treatment. Dis Colon Rectum 2006;49:1837-41.

582 Tanaka S, Matsuo K, Sasaki T, et al. Clinical advantages of combined seton placement and infliximab maintenance therapy for perianal fistulizing Crohn's disease: When and how were the seton drains removed? Hepatogastroenterology 2010;57:3-7.

583 de Groof EJ, Sahami S, Lucas C, et al. Treatment of perianal fistula in Crohn's disease: a systematic review and meta-analysis comparing seton drainage and antitumour necrosis factor treatment. Colorectal Dis 2016;18:667-75.

584 de Groof EJ, Buskens CJ, Ponsioen CY, et al. Multimodal treatment of perianal fistulas in Crohn's disease: seton versus anti-TNF versus advancement plasty (PISA): study protocol for a randomized controlled trial. Trials 2015;16:366.

585 Present DH, Rutgeerts P, Targan S, et al. Infliximab for the treatment of fistulas in patients with Crohn's disease. N Engl J Med 1999;340:1398-405.

586 Yarur AJ, Kanagala V, Stein DJ, et al. Higher infliximab trough levels are associated with perianal fistula healing in patients with Crohn's disease. Aliment Pharmacol Ther 2017;45:933-40

587 Colombel JF, Schwartz DA, Sandborn WJ, et al. Adalimumab for the treatment of fistulas in patients with Crohn's disease. Gut 2009;58:940-8.
588 Dewint $\mathrm{P}$, Hansen BE, Verhey E, et al. Adalimumab combined with ciprofloxacin is superior to adalimumab monotherapy in perianal fistula closure in Crohn's disease: a randomised, double-blind, placebo controlled trial (ADAFI). Gut 2014:63:292-9.

589 Panés J, García-Olmo D, Van Assche G, et al. Expanded allogeneic adipose-derived mesenchymal stem cells (Cx601) for complex perianal fistulas in Crohn's disease: a phase 3 randomised, double-blind controlled trial. Lancet 2016:388:1281-90.

590 Tozer PJ, Lung P, Lobo AJ, et al. Review article: pathogenesis of Crohn's perianal fistula-understanding factors impacting on success and failure of treatment strategies. Aliment Pharmacol Ther 2018;48:260-9.

591 Sands BE, Gasink C, Jacobstein D, et al. Fistula healing in pivotal studies of ustekinumab in Crohn's disease. Gastroenterology 2017;152(5):S185.

592 Tsistrakis S, Oikonomou I. Real-life data on the use of ustekinumab for the treatment of fistulas in patients with Crohn's disease. Am J Gastroenterol 2017;112(Suppl):S361.

593 Lee MJ, Heywood N, Sagar PM, et al. Surgical management of fistulating perianal Crohn's disease: a UK survey. Colorectal Dis 2017;19:266-73.

594 Gingold DS, Murrell ZA, Fleshner PR. A prospective evaluation of the ligation of the intersphincteric tract procedure for complex anal fistula in patients with Crohn's disease. Ann Surg 2014:260:1057-61.

595 van der Hagen SJ, Baeten CG, Soeters PB, et al. Long-term outcome following mucosal advancement flap for high perianal fistulas and fistulotomy for low perianal fistulas: recurrent perianal fistulas: failure of treatment or recurrent patient disease? Int J Colorectal Dis 2006:21:784-90.

596 Schwandner 0. Video-assisted anal fistula treatment (VAAFT) combined with advancement flap repair in Crohn's disease. Tech Coloproctol 2013:17:221-5.

597 O'Riordan JM, Datta I, Johnston C, et al. A systematic review of the anal fistula plug for patients with Crohn's and non-Crohn's related fistula-in-ano. Dis Colon Rectum 2012;55:351-8.

598 Grimaud JC, Munoz-Bongrand N, Siproudhis L, et al. Fibrin glue is effective healing perianal fistulas in patients with Crohn's disease. Gastroenterology 2010;138:2275-81.

599 Garcia-Olmo D, Herreros D, Pascual I, et al. Expanded adipose-derived stem cells for the treatment of complex perianal fistula: a phase II clinical trial. Dis Colon Rectum 2009:52:79-86.

600 de la Portilla F, Alba F, García-Olmo D, et al. Expanded allogeneic adipose-derived stem cells (eASCs) for the treatment of complex perianal fistula in Crohn's disease: results from a multicenter phase I/Ila clinical trial. Int J Colorectal Dis 2013:28:313-23.

601 Panés J, García-Olmo D, Van Assche G, et al. Long-term efficacy and safety of stem cell therapy (cx601) for complex perianal fistulas in patients with crohn's disease. Gastroenterology 2018;154:1334-42.

602 Lightner AL, Wang Z, Zubair AC, et al. A systematic review and meta-analysis of mesenchymal stem cell injections for the treatment of perianal crohn's disease: Progress made and future directions. Dis Colon Rectum 2018;61:629-40.

603 Harper PH, Lee EC, Kettlewell MG, et al. Role of the faecal stream in the maintenance of Crohn's colitis. Gut 1985;26:279-84.

604 Yamamoto T, Allan RN, Keighley MR. Effect of fecal diversion alone on perianal Crohn's disease. World J Surg 2000;24:1258-63.

605 Rehg KL, Sanchez JE, Krieger BR, et al. Fecal diversion in perirectal fistulizing Crohn's disease is an underutilized and potentially temporary means of successful treatment. Am Surg 2009:75:715-8.

606 Edwards CM, George BD, Jewell DP, et al. Role of a defunctioning stoma in the management of large bowel Crohn's disease. Br J Surg 2000;87:1063-6.

607 de Groof EJ, Cabral VN, Buskens CJ, et al. Systematic review of evidence and consensus on perianal fistula: an analysis of national and international guidelines. Colorectal Dis 2016;18:0119-0134.

608 Bafford AC, Latushko A, Hansraj N, et al. The use of temporary fecal diversion in colonic and perianal crohn's disease does not improve outcomes. Dig Dis SCi 2017:62:2079-86.

609 Sauk J, Nguyen D, Yajnik V, et al. Natural history of perianal Crohn's disease after fecal diversion. Inflamm Bowel Dis 2014:20:2260-5.

610 Singh S, Ding NS, Mathis KL, et al. Systematic review with meta-analysis: faecal diversion for management of perianal Crohn's disease. Aliment Pharmacol The 2015;42:783-92.

611 Sailer J, Peloschek P, Reinisch W, et al. Anastomotic recurrence of Crohn's disease after ileocolic resection: comparison of MR enteroclysis with endoscopy. Eur Radio 2008; 18:2512-21.

612 Onali S, Calabrese E, Petruzziello C, et al. Endoscopic vs ultrasonographic findings related to Crohn's disease recurrence: a prospective longitudinal study at 3 years. J Crohns Colitis 2010;4:319-28.

613 Castiglione F, Bucci L, Pesce G, et al. Oral contrast-enhanced sonography for the diagnosis and grading of postsurgical recurrence of Crohn's disease. Inflamm Bowel Dis 2008;14:1240-5. 
614 Biancone L, Calabrese E, Petruzziello C, et al. Wireless capsule endoscopy and small intestine contrast ultrasonography in recurrence of Crohn's disease. Inflamm Bowel Dis 2007;13:1256-65.

615 Calabrese E, Petruzziello C, Onali S, et al. Severity of postoperative recurrence in Crohn's disease: correlation between endoscopic and sonographic findings. Inflamm Bowel Dis 2009;15:1635-42.

616 Paparo F, Revelli M, Puppo C, et al. Crohn's disease recurrence in patients with ileocolic anastomosis: value of computed tomography enterography with water enema. Eur J Radiol 2013;82:e434-40.

617 Soyer P, Boudiaf M, Sirol M, et al. Suspected anastomotic recurrence of Crohn disease after ileocolic resection: evaluation with CT enteroclysis. Radiology 2010;254:755-64

618 Lamb CA, Mohiuddin MK, Gicquel J, et al. Faecal calprotectin or lactoferrin can identify postoperative recurrence in Crohn's disease. Br J Surg 2009;96:663-74.

619 Wright EK, Kamm MA, De Cruz P, et al. Measurement of fecal calprotectin improves monitoring and detection of recurrence of Crohn's disease after surgery. Gastroenterology 2015;148:938-47.

620 Boschetti G, Laidet M, Moussata D, et al. Levels of fecal calprotectin are associated with the severity of postoperative endoscopic recurrence in asymptomatic patients with crohn's disease. Am J Gastroenterol 2015;110:865-72.

621 Mowat C, Arnott I, Cahill A, et al. Mercaptopurine versus placebo to prevent recurrence of Crohn's disease after surgical resection (TOPPIC): a multicentre, double-blind, randomised controlled trial. Lancet Gastroenterol Hepatol 2016:1:273-82.

622 Mottacki N, Simrén M, Bajor A. Review article: bile acid diarrhoea - pathogenesis, diagnosis and management. Aliment Pharmacol Ther 2016;43:884-98.

623 Nyhlin H, Merrick MV, Eastwood MA. Bile acid malabsorption in Crohn's disease and indications for its assessment using SeHCAT. Gut 1994;35:90-3.

624 Kurien M, Evans KE, Leeds JS, et al. Bile acid malabsorption: an under-investigated differential diagnosis in patients presenting with diarrhea predominant irritable bowel syndrome type symptoms. Scand J Gastroentero/ 2011;46:818-22.

625 Gracie DJ, Kane JS, Mumtaz S, et al. Prevalence of, and predictors of, bile acid malabsorption in outpatients with chronic diarrhea. Neurogastroenterol Motil 2012;24:983-e538

626 Jacobsen $\mathrm{O}$, Højgaard L, Hylander Møller E, et al. Effect of enterocoated cholestyramine on bowel habit after ileal resection: a double blind crossover study. Br Med J 1985;290:1315-8.

627 Valdés Olmos R, den Hartog Jager F, Hoefnagel C, et al. Effect of loperamide and delay of bowel motility on bile acid malabsorption caused by late radiation damage and ileal resection. Eur J Nucl Med 1991;18:346-50.

628 Oduyebo I, Camilleri M. Bile acid disease: the emerging epidemic. Curr Opin Gastroenterol 2017;33:189-95.

629 Nolan JD, Johnston I, Dew T, et al. PTU-198 Serum FGF19 Levels are Related to Disease Activity in Ileal Crohn'S Disease. Gut 2013;62(Suppl 1):A130.1.

630 Pattni SS, Brydon WG, Dew T, et al. Fibroblast growth factor 19 in patients with bile acid diarrhoea: a prospective comparison of FGF19 serum assay and SeHCAT retention. Aliment Pharmacol Ther 2013;38:967-76.

631 Brydon WG, Culbert P, Kingstone K, et al. An evaluation of the use of serum 7-alpha-hydroxycholestenone as a diagnostic test of bile acid malabsorption causing watery diarrhea. Can J Gastroenterol 2011;25:319-23.

632 Klaus J, Spaniol U, Adler G, et al. Small intestinal bacterial overgrowth mimicking acute flare as a pitfall in patients with Crohn's Disease. BMC Gastroenterol 2009;9:61

633 Castiglione F, Del Vecchio Blanco G, Rispo A, et al. Orocecal transit time and bacterial overgrowth in patients with Crohn's disease. J Clin Gastroenterol 2000:31:63-6.

634 Bures J, Cyrany J, Kohoutova D, et al. Small intestinal bacterial overgrowth syndrome. World J Gastroenterol 2010;16:2978-90.

635 Shah SC, Day LW, Somsouk M, et al. Meta-analysis: antibiotic therapy for small intestinal bacterial overgrowth. Aliment Pharmacol Ther 2013;38:925-34.

636 Barratt HS, Kalantzis C, Polymeros D, et al. Functional symptoms in inflammatory bowel disease and their potential influence in misclassification of clinical status. Aliment Pharmacol Ther 2005;21:141-7.

637 Reese GE, Nanidis T, Borysiewicz C, et al. The effect of smoking after surgery for Crohn's disease: a meta-analysis of observational studies. Int J Colorectal Dis 2008;23:1213-21.

638 Rutgeerts P, Geboes K, Vantrappen G, et al. Natural history of recurrent Crohn's disease at the ileocolonic anastomosis after curative surgery. Gut 1984:25:665-72.

639 Olaison G, Smedh K, Sjödahl R. Natural course of Crohn's disease after ileocolic resection: endoscopically visualised ileal ulcers preceding symptoms. Gut 1992;33:331-5

640 De Cruz P, Kamm MA, Hamilton AL, et al. Crohn's disease management after intestinal resection: a randomised trial. Lancet 2015;385:1406-17.
641 Orlando A, Mocciaro F, Renna S, et al. Early post-operative endoscopic recurrence in Crohn's disease patients: data from an Italian Group for the study of inflammatory bowel disease (IG-IBD) study on a large prospective multicenter cohort. J Crohns Colitis 2014;8:1217-21.

642 Tytgat GN, Mulder CJ, Brummelkamp WH. Endoscopic lesions in Crohn's disease early after ileocecal resection. Endoscopy 1988;20:260-2.

643 Pascua M, Su C, Lewis JD, et al. Meta-analysis: factors predicting post-operative recurrence with placebo therapy in patients with Crohn's disease. Aliment Pharmacol Ther 2008;28:545-56.

644 Buisson A, Chevaux JB, Allen PB, et al. Review article: the natural history of postoperative Crohn's disease recurrence. Aliment Pharmacol Ther 2012;35:625-33.

645 Milassin Á, Sejben A, Tiszlavicz L, et al. Analysis of risk factors - especially different types of plexitis - for postoperative relapse in Crohn's disease. World J Gastrointest Surg 2017;9:167-73.

646 Simillis C, Jacovides M, Reese GE, et al. Meta-analysis of the role of granulomas in the recurrence of Crohn disease. Dis Colon Rectum 2010;53:177-85.

647 Onali S, Calabrese E, Petruzziello C, et al. Post-operative recurrence of Crohn's disease: A prospective study at 5 years. Dig Liver Dis 2016;48:489-94.

648 Gordon M, Taylor K, Akobeng AK, et al. Azathioprine and 6-mercaptopurine for maintenance of surgically-induced remission in Crohn's disease. Cochrane Database Syst Rev 2014;8:CD010233.

649 Regueiro M, Schraut W, Baidoo L, et al. Infliximab prevents Crohn's disease recurrence after ileal resection. Gastroenterology 2009;136:441-50.

650 Yoshida K, Fukunaga K, Ikeuchi H, et al. Scheduled infliximab monotherapy to prevent recurrence of Crohn's disease following ileocolic or ileal resection: a 3-year prospective randomized open trial. Inflamm Bowel Dis 2012;18:1617-23.

651 Sorrentino D, Terrosu G, Avellini C, et al. Infliximab with low-dose methotrexate for prevention of postsurgical recurrence of ileocolonic Crohn disease. Arch Intern Med 2007; 167:1804-7.

652 Sorrentino D, Paviotti A, Terrosu G, et al. Low-dose maintenance therapy with infliximab prevents postsurgical recurrence of Crohn's disease. Clin Gastroenterol Hepatol 2010;8:591-9.

653 Regueiro M, Feagan BG, Zou B, et al. Infliximab reduces endoscopic, but not clinical, recurrence of Crohn's disease after ileocolonic resection. Gastroenterology 2016:150:1568-78.

654 Collins M, Sarter H, Gower-Rousseau C, et al. Previous exposure to multiple antiTNF is associated with decreased efficiency in preventing postoperative Crohn's disease recurrence. J Crohns Colitis 2017;11:281-8.

655 Savarino E, Bodini G, Dulbecco P, et al. Adalimumab is more effective than azathioprine and mesalamine at preventing postoperative recurrence of Crohn's disease: a randomized controlled trial. Am J Gastroentero/ 2013;108:1731-42.

656 Singh S, Garg SK, Pardi DS, et al. Comparative efficacy of pharmacologic interventions in preventing relapse of Crohn's disease after surgery: a systematic review and network meta-analysis. Gastroenterology 2015;148:64-76.

657 Scribano ML, Prantera C. Use of antibiotics in the treatment of Crohn's disease World J Gastroenterol 2013;19:648-53.

658 Rutgeerts P, Hiele M, Geboes K, et al. Controlled trial of metronidazole treatment for prevention of Crohn's recurrence after ileal resection. Gastroenterology 1995; 108:1617-21.

659 Rutgeerts P, Van Assche G, Vermeire S, et al. Ornidazole for prophylaxis of postoperative Crohn's disease recurrence: a randomized, double-blind, placebocontrolled trial. Gastroenterology 2005;128:856-61.

660 Herfarth HH, Katz JA, Hanauer SB, et al. Ciprofloxacin for the prevention of postoperative recurrence in patients with Crohn's disease: a randomized, doubleblind, placebo-controlled pilot study. Inflamm Bowel Dis 2013;19:1073-9.

661 Jigaranu AO, Nedelciuc O, Blaj A, et al. Is rifaximin effective in maintaining remission in Crohn's disease? Dig Dis 2014;32:378-83.

662 Doherty G, Bennett G, Patil S, et al. Interventions for prevention of post-operative recurrence of Crohn's disease. Cochrane Database Syst Rev 2009:CD006873.

663 Vaughn BP, Moss AC. Prevention of post-operative recurrence of Crohn's disease. World J Gastroenterol 2014;20:1147-54.

664 Yu H, Liu Y, Wang Y, et al. Clinical, endoscopic and histological differentiations between Crohn's disease and intestinal tuberculosis. Digestion 2012;85:202-9.

665 Makharia GK, Srivastava S, Das P, et al. Clinical, endoscopic, and histological differentiations between Crohn's disease and intestinal tuberculosis. Am J Gastroenterol 2010;105:642-51

666 Kirsch R, Pentecost M, Hall PM, et al. Role of colonoscopic biopsy in distinguishing between Crohn's disease and intestinal tuberculosis. J Clin Pathol 2006:59:840-4.

667 Bae JH, Park SH, Ye BD, et al. Development and validation of a novel prediction model for differential diagnosis between Crohn's disease and intestinal tuberculosis. Inflamm Bowel Dis 2017;23:1614-23. 
668 Gan HT, Chen YQ, Ouyang Q, et al. Differentiation between intestinal tuberculosis and Crohn's disease in endoscopic biopsy specimens by polymerase chain reaction. Am J Gastroenterol 2002;97:1446-51.

669 Goodhand JR, Alazawi W, Rampton DS. Systematic review: Clostridium difficile and inflammatory bowel disease. Aliment Pharmacol Ther 2011;33:428-41.

670 Mylonaki M, Langmead L, Pantes A, et al. Enteric infection in relapse of inflammatory bowel disease: importance of microbiological examination of stool. Eur J Gastroenterol Hepatol 2004;16:775-8.

671 Hanada Y, Khanna S, Loftus EV, et al. Non-Clostridium difficile bacterial infections are rare in patients with flares of inflammatory bowel disease. Clin Gastroenterol Hepatol 2018;16:528-33.

672 Axelrad JE, Joelson A, Green PHR, et al. Enteric infections are common in patients with flares of inflammatory bowel disease. Am J Gastroenterol 2018;113:1530-9.

673 Martinelli M, Strisciuglio C, Veres G, et al. Clostridium difficile and pediatric inflammatory bowel disease: a prospective, comparative, multicenter, ESPGHAN study. Inflamm Bowel Dis 2014;20:2219-25.

674 Masclee GM, Penders J, Jonkers DM, et al. Is clostridium difficile associated with relapse of inflammatory bowel disease? results from a retrospective and prospective cohort study in the Netherlands. Inflamm Bowel Dis 2013;19:2125-31.

675 Seril DN, Ashburn JH, Lian L, et al. Risk factors and management of refractory or recurrent clostridium difficile infection in ileal pouch patients. Inflamm Bowel Dis 2014;20:2226-33

676 Jodorkovsky D, Young Y, Abreu MT. Clinical outcomes of patients with ulcerative colitis and co-existing Clostridium difficile infection. Dig Dis Sci 2010;55:415-20.

677 Ananthakrishnan AN, McGinley EL, Binion DG. Excess hospitalisation burden associated with Clostridium difficile in patients with inflammatory bowel disease. Gut 2008:57:205-10.

678 Ananthakrishnan AN, McGinley EL, Saeian K, et al. Temporal trends in disease outcomes related to Clostridium difficile infection in patients with inflammatory bowel disease. Inflamm Bowel Dis 2011;17:976-83.

679 Law CC, Tariq R, Khanna S, et al. Systematic review with meta-analysis: the impact of Clostridium difficile infection on the short- and long-term risks of colectomy in inflammatory bowel disease. Aliment Pharmacol Ther 2017;45:1011-20.

680 Zar FA, Bakkanagari SR, Moorthi KM, et al. A comparison of vancomycin and metronidazole for the treatment of Clostridium difficile-associated diarrhea, stratified by disease severity. Clin Infect Dis 2007:45:302-7.

681 Khanna S, Shin A, Kelly CP. Management of Clostridium difficile infection in inflammatory bowel disease: expert review from the Clinical Practice Updates Committee of the AGA Institute. Clin Gastroenterol Hepatol 2017;15:166-74.

682 Tandon P, James P, Cordeiro E, et al. Diagnostic accuracy of blood-based tests and histopathology for cytomegalovirus reactivation in inflammatory bowel disease: a systematic review and meta-analysis. Inflamm Bowel Dis 2017;23:551-60.

683 McCurdy JD, Jones A, Enders FT, et al. A model for identifying cytomegalovirus in patients with inflammatory bowel disease. Clin Gastroenterol Hepatol 2015;13:131-7.

684 Maconi G, Colombo E, Zerbi P, et al. Prevalence, detection rate and outcome of cytomegalovirus infection in ulcerative colitis patients requiring colonic resection. Dig Liver Dis 2005;37:418-23.

685 Domènech E, Vega R, Ojanguren I, et al. Cytomegalovirus infection in ulcerative colitis: a prospective, comparative study on prevalence and diagnostic strategy. Inflamm Bowel Dis 2008:14:1373-9.

686 Lv YL, Han FF, Jia YJ, et al. Is cytomegalovirus infection related to inflammatory bowel disease, especially steroid-resistant inflammatory bowel disease? A metaanalysis. Infect Drug Resist 2017;10:511-9.

687 Gauss A, Rosenstiel S, Schnitzler P, et al. Intestinal cytomegalovirus infection in patients hospitalized for exacerbation of inflammatory bowel disease: a 10-year tertiary referral center experience. Eur J Gastroenterol Hepatol 2015;27:712-20.

$688 \mathrm{Kim} \mathrm{JW}, \mathrm{Boo} \mathrm{SJ}$, Ye BD, et al. Clinical utility of cytomegalovirus antigenemia assay and blood cytomegalovirus DNA PCR for cytomegaloviral colitis patients with moderate to severe ulcerative colitis. J Crohns Colitis 2014;8:693-701.

689 Yoshino $\mathrm{T}$, Nakase $\mathrm{H}$, Ueno $\mathrm{S}$, et al. Usefulness of quantitative real-time PCR assay for early detection of cytomegalovirus infection in patients with ulcerative colitis refractory to immunosuppressive therapies. Inflamm Bowel Dis 2007;13:1516-21.

690 Criscuoli V, Rizzuto MR, Cottone M. Cytomegalovirus and inflammatory bowel disease: is there a link? World J Gastroenterol 2006;12:4813-8.

691 Vega R, Bertrán X, Menacho M, et al. Cytomegalovirus infection in patients with inflammatory bowel disease. Am J Gastroenterol 1999:94:1053-6.

692 Rahbar A, Boström L, Lagerstedt U, et al. Evidence of active cytomegalovirus infection and increased production of IL-6 in tissue specimens obtained from patients with inflammatory bowel diseases. Inflamm Bowel Dis 2003:9:154-61.

693 Römkens TE, Bulte GJ, Nissen LH, et al. Cytomegalovirus in inflammatory bowel disease: A systematic review. World J Gastroenterol 2016;22:1321-30.
694 Matsuoka K, Iwao Y, Mori T, et al. Cytomegalovirus is frequently reactivated and disappears without antiviral agents in ulcerative colitis patients. Am I Gastroenterol 2007:102:331-7.

$695 \mathrm{Kim}$ YS, Kim YH, Kim JS, et al. The prevalence and efficacy of ganciclovir on steroid-refractory ulcerative colitis with cytomegalovirus infection: a prospective multicenter study. J Clin Gastroenterol 2012;46:51-6.

696 Roblin X, Pillet S, Oussalah A, et al. Cytomegalovirus load in inflamed intestinal tissue is predictive of resistance to immunosuppressive therapy in ulcerative colitis. Am J Gastroenterol 2011;106:2001-8.

697 Kopylov U, Papamichael K, Katsanos K, et al. Impact of infliximab and cyclosporine on the risk of colectomy in hospitalized patients with ulcerative colitis complicated by cytomegalovirus: a multicenter retrospective study. Inflamm Bowel Dis 2017:23:1605-13.

698 Jones A, McCurdy JD, Loftus EV, et al. Effects of antiviral therapy for patients with inflammatory bowel disease and a positive intestinal biopsy for cytomegalovirus. Clin Gastroenterol Hepatol 2015;13:949-55.

699 Sager K, Alam S, Bond A, et al. Review article: cytomegalovirus and inflammatory bowel disease. Aliment Pharmacol Ther 2015;41:725-33.

700 Rahier JF, Magro F, Abreu C, et al. Second European evidence-based consensus on the prevention, diagnosis and management of opportunistic infections in inflammatory bowel disease. J Crohns Colitis 2014;8:443-68.

701 Broekman M, Coenen MJH, Wanten GJ, et al. Patients' beliefs about medicine are associated with early thiopurine discontinuation in patients with inflammatory bowel diseases. Eur I Gastroenterol Hepatol 2018:30:167-73.

702 Selinger CP, Carbery I, Warren V, et al. The relationship between different information sources and disease-related patient knowledge and anxiety in patients with inflammatory bowel disease. Aliment Pharmacol Ther 2017;45:63-74.

703 Chevaux JB, Nani A, Oussalah A, et al. Prevalence of hepatitis B and C and risk factors for nonvaccination in inflammatory bowel disease patients in Northeast France. Inflamm Bowel Dis 2010;16:916-24.

704 van der Have M, Belderbos TD, Fidder HH, et al. Screening prior to biological therapy in Crohn's disease: adherence to guidelines and prevalence of infections. Results from a multicentre retrospective study. Dig Liver Dis 2014;46:881-6.

705 de Francisco R, Castaño-García A, Martínez-González S, et al. Impact of Epstein-Barr virus serological status on clinical outcomes in adult patients with inflammatory bowel disease. Aliment Pharmacol Ther 2018;48:723-30.

706 Hyams JS, Dubinsky MC, Baldassano RN, et al. Infliximab is not associated with increased risk of malignancy or hemophagocytic lymphohistiocytosis in pediatric patients with inflammatory bowel disease. Gastroenterology 2017;152:1901-14.

707 Barnes EL, Herfarth HH. The usefulness of serologic testing for Epstein-Barr virus before initiation of therapy for inflammatory bowel disease. Gastroenterology 2017;153:1167.

708 Ford AC, Peyrin-Biroulet L. Opportunistic infections with anti-tumor necrosis factor- $\alpha$ therapy in inflammatory bowel disease: meta-analysis of randomized controlled trials. Am I Gastroenterol 2013:108:1268-76.

709 Osterman MT, Sandborn WJ, Colombel JF, et al. Crohn's disease activity and concomitant immunosuppressants affect the risk of serious and opportunistic infections in patients treated with adalimumab. Am J Gastroenterol 2016;111:1806-15.

710 Naganuma M, Kunisaki R, Yoshimura N, et al. A prospective analysis of the incidence of and risk factors for opportunistic infections in patients with inflammatory bowel disease. J Gastroenterol 2013:48:595-600.

711 Toruner M, Loftus EV, Harmsen WS, et al. Risk factors for opportunistic infections in patients with inflammatory bowel disease. Gastroenterology 2008;134:929-36.

712 Mow WS, Abreu-Martin MT, Papadakis KA, et al. High incidence of anergy in inflammatory bowel disease patients limits the usefulness of PPD screening before infliximab therapy. Clin Gastroenterol Hepatol 2004;2:309-13.

713 Qumseya BJ, Ananthakrishnan AN, Skaros S, et al. QuantiFERON TB gold testing for tuberculosis screening in an inflammatory bowel disease cohort in the United States. Inflamm Bowel Dis 2011;17:77-83.

714 Greveson K, Goodhand J, Capocci S, et al. Yield and cost effectiveness of mycobacterial infection detection using a simple IGRA-based protocol in UK subjects with inflammatory bowel disease suitable for anti-TNF $\alpha$ therapy. J Crohns Colitis 2013:7:412-8

715 British Thoracic Society Standards of Care Committee. BTS recommendations for assessing risk and for managing Mycobacterium tuberculosis infection and disease in patients due to start anti-TNF-alpha treatment. Thorax 2005;60:800-5.

716 Byun JM, Lee CK, Rhee SY, et al. Risks for opportunistic tuberculosis infection in a cohort of 873 patients with inflammatory bowel disease receiving a tumor necrosis factor- $\alpha$ inhibitor. Scand I Gastroenterol 2015:50:312-20.

717 van der Have M, Oldenburg B, Fidder HH, et al. Optimizing screening for tuberculosis and hepatitis B prior to starting tumor necrosis factor- $\alpha$ inhibitors in Crohn's disease. Dig Dis Sci 2014;59:554-63. 
718 Schär F, Trostdorf U, Giardina F, et al. Strongyloides stercoralis: global distribution and risk factors. PLoS Negl Trop Dis 2013;7:e2288.

719 Keiser PB, Nutman TB. Strongyloides stercoralis in the immunocompromised population. Clin Microbiol Rev 2004;17:208-17.

720 Loutfy MR, Wilson M, Keystone JS, et al. Serology and eosinophil count in the diagnosis and management of strongyloidiasis in a non-endemic area. Am J Trop Med Hyg 2002;66:749-52.

721 Tinsley A, Navabi S, Williams ED, et al. Increased risk of influenza and influenzarelated complications among 140,480 patients with inflammatory bowel disease. Inflamm Bowel Dis 2019;25:369-76.

722 Department of Health. Influenza: The Green Book, Chapter 19. 2018. https://www. gov.uk/government/publications/influenza-the-green-book-chapter-19 (Accessed 7 Feb 2019).

723 deBruyn J, Fonseca K, Ghosh S, et al. Immunogenicity of influenza vaccine for patients with inflammatory bowel disease on maintenance infliximab therapy: a randomized trial. Inflamm Bowel Dis 2016:22:638-47.

724 Department of Health. Hepatitis B: The Green Book, Chapter 18. July 2017 https:// www.gov.uk/government/publications/hepatitis-b-the-green-book-chapter-18 (Accessed 14 Dec 2018).

725 Belle A, Baumann C, Bigard MA, et al. Impact of immunosuppressive therapy on hepatitis B vaccination in inflammatory bowel diseases. Eur J Gastroenterol Hepatol 2015:27:877-81.

726 Andrade P, Santos-Antunes J, Rodrigues S, et al. Treatment with infliximab or azathioprine negatively impact the efficacy of hepatitis $B$ vaccine in inflammatory bowel disease patients. J Gastroenterol Hepatol 2015;30:1591-5.

727 Altunöz ME, Senateș E, Yeșil A, et al. Patients with inflammatory bowel disease have a lower response rate to HBV vaccination compared to controls. Dig Dis Sci 2012:57:1039-44

728 Gisbert JP, Menchén L, García-Sánchez V, et al. Comparison of the effectiveness of two protocols for vaccination (standard and double dosage) against hepatitis $B$ virus in patients with inflammatory bowel disease. Aliment Pharmacol Ther 2012;35:1379-85

729 Department of Health. Pneumococcal: The Green Book, Chapter 25. January 2018 https://www.gov.uk/government/publications/pneumococcal-the-green-bookchapter-25 (Accessed 14 Dec 2018)

730 Department of Health. Contraindications and special considerations: The Green Book, Chapter 6. October 2017 https://www.gov.uk/government/publications/ contraindications-and-special-considerations-the-green-book-chapter-6 (Accessed 14 Dec 2018)

731 Reich J, Wasan S, Farraye FA. Vaccinating patients with inflammatory bowel disease. Gastroenterol Hepatol 2016;12:540-6.

732 Chaudrey K, Salvaggio M, Ahmed A, et al. Updates in vaccination: recommendations for adult inflammatory bowel disease patients. World 」 Gastroenterol 2015;21:3184-96.

733 Rahier JF, Moutschen M, Van Gompel A, et al. Vaccinations in patients with immune-mediated inflammatory diseases. Rheumatology 2010;49:1815-27.

734 Rubin LG, Levin MJ, Ljungman P, et al. IDSA clinical practice guideline for vaccination of the immunocompromised host. Clin Infect Dis 2013:2014:e44-100.

735 Department of Health. Shingles (herpes zoster): The Green Book, Chapter 28A. 2016. https://www.gov.uk/government/publications/shingles-herpes-zoster-thegreen-book-chapter-28a (Accessed 10 Jan 2019).

736 Wasan SK, Zullow S, Berg A, et al. Herpes zoster vaccine response in inflammatory bowel disease patients on low-dose immunosuppression. Inflamm Bowel Dis 2016;22:1391-6

737 Coenen MJ, de Jong DJ, van Marrewijk CJ, et al. Identification of patients with variants in TPMT and dose reduction reduces hematologic events during thiopurine treatment of inflammatory bowel disease. Gastroenterology 2015;149:907-17

738 Ansari A, Arenas M, Greenfield SM, et al. Prospective evaluation of the pharmacogenetics of azathioprine in the treatment of inflammatory bowel disease. Aliment Pharmacol Ther 2008;28:973-83.

739 Winter J, Walker A, Shapiro D, et al. Cost-effectiveness of thiopurine methyltransferase genotype screening in patients about to commence azathioprine therapy for treatment of inflammatory bowel disease. Aliment Pharmacol Ther 2004;20:593-9

740 Priest VL, Begg EJ, Gardiner SJ, et al. Pharmacoeconomic analyses of azathioprine, methotrexate and prospective pharmacogenetic testing for the management of inflammatory bowel disease. Pharmacoeconomics 2006;24:767-81.

741 Yang SK, Hong M, Baek J, et al. A common missense variant in NUDT15 confers susceptibility to thiopurine-induced leukopenia. Nat Genet 2014;46:1017-20.

742 Relling MV, Schwab M, Whirl-Carrillo M, et al. Clinical Pharmacogenetics Implementation Consortium guideline for thiopurine dosing based on TPMT and NUDT15 genotypes: 2018 update. Clin Pharmacol Ther 2019;105:1095-105.
743 Yip JS, Woodward M, Abreu MT, et al. How are Azathioprine and 6-mercaptopurine dosed by gastroenterologists? Results of a survey of clinical practice. Inflamm Bowel Dis 2008;14:514-8.

744 Aronoff GR, Bennett WB, Berns IS, et al. Drug Prescribing in Renal Failure: Dosing Guidelines for Adults and Children: American College of Physicians. 2007.

745 Rungoe C, Simonsen J, Riis L, et al. Inflammatory bowel disease and cervical neoplasia: a population-based nationwide cohort study. Clin Gastroenterol Hepatol 2015;13:693-700

746 Kim SC, Glynn RJ, Giovannucci E, et al. Risk of high-grade cervical dysplasia and cervical cancer in women with systemic inflammatory diseases: a population-based cohort study. Ann Rheum Dis 2015;74:1360-7.

747 Singh $H$, Demers AA, Nugent Z, et al. Risk of cervical abnormalities in women with inflammatory bowel disease: a population-based nested case-control study. Gastroenterology 2009;136:451-8.

748 Lees CW, Critchley J, Chee N, et al. Lack of association between cervical dysplasia and IBD: a large case-control study. Inflamm Bowel Dis 2009;15:1621-9.

749 Kane S, Khatibi B, Reddy D. Higher incidence of abnormal Pap smears in women with inflammatory bowel disease. Am J Gastroenterol 2008;103:631-6.

750 Allegretti JR, Barnes EL, Cameron A. Are patients with inflammatory bowel disease on chronic immunosuppressive therapy at increased risk of cervical high-grade dysplasia/cancer? A meta-analysis. Inflamm Bowel Dis 2015;21:1089-97.

751 Smith M, Blaker P, Patel C, et al. The impact of introducing thioguanine nucleotide monitoring into an inflammatory bowel disease clinic. Int I Clin Pract 2013:67:161-9.

752 Haines ML, Ajlouni Y, Irving PM, et al. Clinical usefulness of therapeutic drug monitoring of thiopurines in patients with inadequately controlled inflammatory bowel disease. Inflamm Bowel Dis 2011;17:1301-7.

753 Dubinsky MC, Reyes E, Ofman J, et al. A cost-effectiveness analysis of alternative disease management strategies in patients with Crohn's disease treated with azathioprine or 6-mercaptopurine. Am J Gastroenterol 2005; 100:2239-47.

754 Wright S, Sanders DS, Lobo AJ, et al. Clinical significance of azathioprine active metabolite concentrations in inflammatory bowel disease. Gut 2004;53:1123-8.

755 Dubinsky MC, Lamothe S, Yang HY, et al. Pharmacogenomics and metabolite measurement for 6-mercaptopurine therapy in inflammatory bowel disease. Gastroenterology 2000;118:705-13.

756 Reinshagen M, Schütz E, Armstrong VW, et al. 6-thioguanine nucleotide-adapted azathioprine therapy does not lead to higher remission rates than standard therapy in chronic active crohn disease: results from a randomized, controlled, open trial. Clin Chem 2007;53:1306-14.

757 González-Lama Y, Bermejo F, López-Sanromán A, et al. Thiopurine methyltransferase activity and azathioprine metabolite concentrations do not predict clinical outcome in thiopurine-treated inflammatory bowel disease patients. Aliment Pharmacol Ther 2011;34:544-54.

758 Dassopoulos T, Dubinsky MC, Bentsen JL, et al. Randomised clinical trial: individualised vs. weight-based dosing of azathioprine in Crohn's disease. Aliment Pharmacol Ther 2014:39:163-75.

759 Yarur AJ, Kubiliun MJ, Czul F, et al. Concentrations of 6-thioguanine nucleotide correlate with trough levels of infliximab in patients with inflammatory bowel disease on combination therapy. Clin Gastroenterol Hepatol 2015;13:1118-24.

760 Roblin X, Boschetti G, Williet N, et al. Azathioprine dose reduction in inflammatory bowel disease patients on combination therapy: an open-label, prospective and randomised clinical trial. Aliment Pharmacol Ther 2017;46:142-9.

761 Dubinsky MC, Yang H, Hassard PV, et al. 6-MP metabolite profiles provide a biochemical explanation for 6-MP resistance in patients with inflammatory bowe disease. Gastroenterology 2002;122:904-15.

762 Blaker PA, Arenas-Hernandez M, Smith MA, et al. Mechanism of allopurinol induced TPMT inhibition. Biochem Pharmacol 2013:86:539-47.

763 Friedman AB, Brown SJ, Bampton P, et al. Randomised clinical trial: efficacy, safety and dosage of adjunctive allopurinol in azathioprine/mercaptopurine nonresponders (AAA Study). Aliment Pharmacol Ther 2018;47:1092-102.

764 Derijks LJ, Gilissen LP, Engels LG, et al. Pharmacokinetics of 6-mercaptopurine in patients with inflammatory bowel disease: implications for therapy. Ther Drug Monit 2004;26:311-8.

765 Kiszka-Kanowitz M, Theede K, Mertz-Nielsen A. Randomized clinical trial: a pilot study comparing efficacy of low-dose azathioprine and allopurinol to azathioprine on clinical outcomes in inflammatory bowel disease. Scand I Gastroenterol 2016;51:1470-5.

766 Smith MA, Blaker P, Marinaki AM, et al. Optimising outcome on thiopurines in inflammatory bowel disease by co-prescription of allopurinol. J Crohns Colitis 2012;6:905-12.

767 Ansari A, Elliott T, Baburajan B, et al. Long-term outcome of using allopurinol co-therapy as a strategy for overcoming thiopurine hepatotoxicity in treating inflammatory bowel disease. Aliment Pharmacol Ther 2008;28:734-41. 
768 Ansari A, Patel N, Sanderson J, et al. Low-dose azathioprine or mercaptopurine in combination with allopurinol can bypass many adverse drug reactions in patients with inflammatory bowel disease. Aliment Pharmacol Ther 2010;31:640-7.

769 Vasudevan A, Beswick L, Friedman AB, et al. Low-dose thiopurine with allopurinol co-therapy overcomes thiopurine intolerance and allows thiopurine continuation in inflammatory bowel disease. Dig Liver Dis 2018;50:682-8.

770 Sparrow MP, Hande SA, Friedman S, et al. Effect of allopurinol on clinical outcomes in inflammatory bowel disease nonresponders to azathioprine or 6-mercaptopurine. Clin Gastroenterol Hepatol 2007;5:209-14.

771 Leung Y, Sparrow MP, Schwartz M, et al. Long term efficacy and safety of allopurinol and azathioprine or 6-mercaptopurine in patients with inflammatory bowel disease. J Crohns Colitis 2009;3:162-7

772 Friedman AB, Brown SJ, Bampton P, et al. Randomised clinical trial: efficacy, safety and dosage of adjunctive allopurinol in azathioprine/mercaptopurine nonresponders (AAA Study). Aliment Pharmacol Ther 2018;47:1092-102.

773 Colombel JF, Ferrari N, Debuysere H, et al. Genotypic analysis of thiopurine S-methyltransferase in patients with Crohn's disease and severe myelosuppression during azathioprine therapy. Gastroenterology 2000;118:1025-30.

774 Lewis JD, Abramson 0, Pascua M, et al. Timing of myelosuppression during thiopurine therapy for inflammatory bowel disease: implications for monitoring recommendations. Clin Gastroenterol Hepatol 2009;7:1195-201.

775 Gisbert JP, Gomollón F. Thiopurine-induced myelotoxicity in patients with inflammatory bowel disease: a review. Am J Gastroenterol 2008; 103:1783-800.

776 Chaparro M, Ordás I, Cabré E, et al. Safety of thiopurine therapy in inflammatory bowel disease: long-term follow-up study of 3931 patients. Inflamm Bowel Dis 2013;19:1404-10.

777 Heap GA, Weedon MN, Bewshea CM, et al. HLA-DQA1-HLA-DRB1 variants confer susceptibility to pancreatitis induced by thiopurine immunosuppressants. Nat Genet 2014;46:1131-4.

778 Kennedy NA, Rhatigan E, Arnott ID, et al. A trial of mercaptopurine is a safe strategy in patients with inflammatory bowel disease intolerant to azathioprine: an observational study, systematic review and meta-analysis. Aliment Pharmacol Ther 2013;38:1255-66.

779 Whittle SL, Hughes RA. Folate supplementation and methotrexate treatment in rheumatoid arthritis: a review. Rheumatology 2004;43:267-71.

780 Ortiz Z, Shea B, Suarez-Almazor ME, et al. The efficacy of folic acid and folinic acid in reducing methotrexate gastrointestinal toxicity in rheumatoid arthritis. $A$ metaanalysis of randomized controlled trials. J Rheumatol 1998;25:36-43.

781 Herfarth $\mathrm{HH}$, Kappelman MD, Long MD, et al. Use of methotrexate in the treatment of inflammatory bowel diseases. Inflamm Bowel Dis 2016:22:224-33.

782 González-Lama Y, Taxonera C, López-Sanromán A, et al. Methotrexate in inflammatory bowel disease: a multicenter retrospective study focused on longterm efficacy and safety. The Madrid experience. Eur J Gastroenterol Hepatol 2012;24:1086-91.

783 Fournier MR, Klein J, Minuk GY, et al. Changes in liver biochemistry during methotrexate use for inflammatory bowel disease. Am J Gastroenterol 2010;105:1620-6.

784 Laharie D, Seneschal J, Schaeverbeke T, et al. Assessment of liver fibrosis with transient elastography and FibroTest in patients treated with methotrexate for chronic inflammatory diseases: a case-control study. J Hepatol 2010;53:1035-40.

785 Østensen M, Khamashta M, Lockshin M, et al. Anti-inflammatory and immunosuppressive drugs and reproduction. Arthritis Res Ther 2006;8:209.

786 Panchal S, Flint J, van de Venne M, et al. A systematic analysis of the safety of prescribing of anti-rheumatic, immunosuppressive and biologic drugs in men trying to conceive. Ann Rheum Dis 2014;73(Suppl 2):415.2-415.

787 Weber-Schoendorfer C, Hoeltzenbein M, Wacker E, et al. No evidence for an increased risk of adverse pregnancy outcome after paternal low-dose methotrexate: an observational cohort study. Rheumatology 2014;53:757-63.

788 Winter RW, Larsen MD, Magnussen B, et al. Birth outcomes after preconception paternal exposure to methotrexate: A nationwide cohort study. Reprod Toxicol 2017:74:219-23.

789 Stidham RW, Lee TC, Higgins PD, et al. Systematic review with network metaanalysis: the efficacy of anti-TNF agents for the treatment of Crohn's disease. Aliment Pharmacol Ther 2014;39:1349-62.

790 Cholapranee A, Hazlewood GS, Kaplan GG, et al. Systematic review with metaanalysis: comparative efficacy of biologics for induction and maintenance of mucosal healing in Crohn's disease and ulcerative colitis controlled trials. Aliment Pharmacol Ther 2017;45:1291-302.

791 Mao EJ, Hazlewood GS, Kaplan GG, et al. Systematic review with meta-analysis: comparative efficacy of immunosuppressants and biologics for reducing hospitalisation and surgery in Crohn's disease and ulcerative colitis. Aliment Pharmacol Ther 2017;45:3-13.
792 Moćko P, Kawalec P, Pilc A. Safety profile of biologic drugs in the therapy of Crohn disease: A systematic review and network meta-analysis. Pharmacol Rep 2016:68:1237-43.

793 Osterman MT, Haynes K, Delzell E, et al. Effectiveness and safety of immunomodulators with anti-tumor necrosis factor therapy in Crohn's disease. Clin Gastroenterol Hepatol 2015;13:1293-301.

794 Singh S, Heien HC, Sangaralingham LR, et al. Comparative effectiveness and safety of anti-tumor necrosis factor agents in biologic-naive patients with Crohn's disease. Clin Gastroenterol Hepatol 2016;14:1120-9.

795 Singh S, Andersen NN, Andersson M, et al. Comparison of infliximab and adalimumab in biologic-naive patients with ulcerative colitis: a nationwide Danish cohort study. Clin Gastroenterol Hepatol 2017;15:1218-25.

796 Van Assche G, Vermeire S, Ballet V, et al. Switch to adalimumab in patients with Crohn's disease controlled by maintenance infliximab: prospective randomised SWITCH trial. Gut 2012;61:229-34.

797 Vavricka SR, Bentele N, Scharl M, et al. Swiss IBDnet and Swiss IBD Cohort Study Group. Systematic assessment of factors influencing preferences of Crohn's disease patients in selecting an anti-tumor necrosis factor agent (CHOOSE TNF TRIAL). Inflamm Bowel Dis 2012;18:1523-30.

798 Kim ES, Kim KO, Jang BI, et al. IBD Study Group of the Korean Association for the Study of the Intestinal Diseases (KASID). Factors contributing to the preference of Korean patients with Crohn's disease when selecting an anti-tumor necrosis factor agent (CHOICE Study). Gut Liver 2016;10:391-8.

799 European Medicines Agency. Biosimilars in the EU: Information guide for healthcare professionals. 2017. https://www.ema.europa.eu/documents/leaflet/ biosimilars-eu-information-guide-healthcare-professionals_en.pdf [Accessed 14 Dec 2018].

800 Association of the British Pharmaceutical Industry. ABPI position on biologic medicines, including biosimilar medicines. 2015. https://www.abpi.org.uk/media/ 4568/abpi-position-on-biosimilar-medicines.pdf [Accessed 14 Dec 2018].

801 Moots R, Azevedo V, Coindreau JL, et al. Switching between reference biologics and biosimilars for the treatment of rheumatology, gastroenterology, and dermatology inflammatory conditions: considerations for the clinician. Curr Rheumatol Rep 2017; $19: 37$

802 Danese S, Fiorino G, Raine T, et al. ECCO position statement on the use of biosimilars for inflammatory bowel disease-an update. J Crohns Colitis 2017:11:26-34

803 Jørgensen KK, Olsen IC, Goll GL, et al. NOR-SWITCH study group. Switching from originator infliximab to biosimilar CT-P13 compared with maintained treatment with originator infliximab (NOR-SWITCH): a 52-week, randomised, double-blind, non-inferiority trial. Lancet 2017;389:2304-16.

804 Buer LC, Moum BA, Cvancarova M, et al. Switching from Remicade ${ }^{\circledR}$ to Remsima $($ is well tolerated and feasible: a prospective, open-label study. J Crohns Colitis 2017;11:297-304.

805 Smits LJ, Derikx LA, de Jong DJ, et al. Clinical outcomes following a switch from Remicade $®$ to the biosimilar CT-P13 in inflammatory bowel disease patients: a prospective observational cohort study. J Crohns Colitis 2016;10:1287-93.

806 Dapavo P, Vujic I, Fierro MT, et al. The infliximab biosimilar in the treatment of moderate to severe plaque psoriasis. J Am Acad Dermatol 2016;75:736-9.

807 Park W, Yoo DH, Miranda P, et al. Efficacy and safety of switching from reference infliximab to CT-P13 compared with maintenance of CT-P13 in ankylosing spondylitis: 102-week data from the PLANETAS extension study. Ann Rheum Dis 2017;76:346-54.

808 Yoo DH, Prodanovic N, Jaworski J, et al. Efficacy and safety of CT-P13 (biosimilar infliximab) in patients with rheumatoid arthritis: comparison between switching from reference infliximab to CT-P13 and continuing CT-P13 in the PLANETRA extension study. Ann Rheum Dis 2017;76:355-63.

809 Glintborg B, Sørensen IJ, Loft AG, et al. All Departments of Rheumatology in Denmark. A nationwide non-medical switch from originator infliximab to biosimilar CT-P13 in 802 patients with inflammatory arthritis: 1-year clinical outcomes from the DANBIO registry. Ann Rheum Dis 2017;76:1426-31.

810 D'Haens G, Vermeire S, Lambrecht G, et al. GETAID. Increasing infliximab dose based on symptoms, biomarkers, and serum drug concentrations does not increase clinical, endoscopic, and corticosteroid-free remission in patients with active luminal Crohn's disease. Gastroenterology 2018;154:1343-51.

811 Papamichael K, Vande Casteele N, Ferrante M, et al. Therapeutic drug monitoring during induction of anti-tumor necrosis factor therapy in inflammatory bowel disease: defining a therapeutic drug window. Inflamm Bowel Dis 2017:23:1510-5.

812 Ding NS, Hart A, De Cruz P. Systematic review: predicting and optimising response to anti-TNF therapy in Crohn's disease - algorithm for practical management. Aliment Pharmacol Ther 2016;43:30-51.

813 Papamichael K, Cheifetz AS. Therapeutic drug monitoring in IBD: the new standardof-care for anti-TNF therapy. Am J Gastroenterol 2017;112:673-6. 
814 Yarur AJ, Rubin DT. Therapeutic drug monitoring of anti-tumor necrosis factor agents in patients with inflammatory bowel diseases. Inflamm Bowel Dis 2015;21:1709-18.

815 Vaughn BP, Sandborn WJ, Cheifetz AS. Biologic concentration testing in inflammatory bowel disease. Inflamm Bowel Dis 2015;21:1-42.

816 Vande Casteele N, Ferrante M, Van Assche G, et al. Trough concentrations of infliximab guide dosing for patients with inflammatory bowel disease. Gastroenterology 2015;148:1320-9.

817 Roblin X, Marotte H, Rinaudo M, et al. Association between pharmacokinetics of adalimumab and mucosal healing in patients with inflammatory bowel diseases. Clin Gastroenterol Hepatol 2014;12:80-4.

818 Steenholdt C, Brynskov J, Thomsen 0Ø, et al. Individualised therapy is more costeffective than dose intensification in patients with Crohn's disease who lose response to anti-TNF treatment: a randomised, controlled trial. Gut 2014;63:919-27.

819 Yanai $H$, Lichtenstein L, Assa A, et al. Levels of drug and antidrug antibodies are associated with outcome of interventions after loss of response to infliximab or adalimumab. Clin Gastroenterol Hepatol 2015;13:522-30.

820 Velayos FS, Kahn JG, Sandborn WJ, et al. A test-based strategy is more cost effective than empiric dose escalation for patients with Crohn's disease who lose responsiveness to infliximab. Clin Gastroenterol Hepatol 2013;11:654-66.

821 Paul S, Del Tedesco E, Marotte $\mathrm{H}$, et al. Therapeutic drug monitoring of infliximab and mucosal healing in inflammatory bowel disease: a prospective study. Inflamm Bowel Dis 2013;19:2568-76.

822 Kopylov U, Mantzaris GJ, Katsanos KH, et al. The efficacy of shortening the dosing interval to once every six weeks in Crohn's patients losing response to maintenance dose of infliximab. Aliment Pharmacol Ther 2011;33:349-57.

823 Katz L, Gisbert JP, Manoogian B, et al. Doubling the infliximab dose versus halving the infusion intervals in Crohn's disease patients with loss of response. Inflamm Bowel Dis 2012;18:2026-33.

824 Vande Casteele N, Gils A, Singh S, et al. Antibody response to infliximab and its impact on pharmacokinetics can be transient. Am J Gastroenterol 2013;108:962-71.

825 Van Stappen T, Vande Casteele N, Van Assche G, et al. Clinical relevance of detecting anti-infliximab antibodies with a drug-tolerant assay: post hoc analysis of the TAXIT trial. Gut 2018;67:818-26.

826 Ben-Horin S, Waterman M, Kopylov U, et al. Addition of an immunomodulator to infliximab therapy eliminates antidrug antibodies in serum and restores clinical response of patients with inflammatory bowel disease. Clin Gastroenterol Hepatol 2013;11:444-7.

827 Bartelds GM, Wijbrandts CA, Nurmohamed MT, et al. Anti-infliximab and antiadalimumab antibodies in relation to response to adalimumab in infliximab switchers and anti-tumour necrosis factor naive patients: a cohort study. Ann Rheum Dis 2010;69:817-21.

828 Gils A. Combining therapeutic drug monitoring with biosimilars, a strategy to improve the efficacy of biologicals for treating inflammatory bowel diseases at an affordable cost. Dig Dis 2017;35:61-8.

829 National Institute for Health and Care Excellence. Technology appraisal guidance [TA456] Ustekinumab for moderately to severely active Crohn's disease after previous treatment. https://www.nice.org.uk/guidance/ta456 [Accessed 17 Jan 2019].

830 National Institute for Health and Care Excellence. Technology appraisal guidance [TA187] Infliximab and adalimumab for the treatment of Crohn's disease. 2010. https://www.nice.org.uk/guidance/ta187 [Accessed 15 Dec 2018].

831 National Institute for Health and Care Excellence. Technology appraisal guidance [TA342] Vedolizumab for treating moderately to severely active ulcerative colitis. 2015. https://www.nice.org.uk/guidance/ta342 [Accessed 15 Dec 2018].

832 National Institute for Health and Care Excellence. Technology appraisal guidance [TA352] Vedolizumab for treating moderately to severely active Crohn's disease after prior therapy. https://www.nice.org.uk/guidance/ta352 [Accessed 17 Jan 2019].

833 Jin Y, Lin Y, Lin LJ, et al. Meta-analysis of the effectiveness and safety of vedolizumab for ulcerative colitis. World I Gastroenterol 2015;21:6352-60.

834 Luthra P, Peyrin-Biroulet L, Ford AC. Systematic review and meta-analysis: opportunistic infections and malignancies during treatment with antiintegrin antibodies in inflammatory bowel disease. Aliment Pharmacol Ther 2015;41:1227-36

835 Ge WS, Fan JG. Integrin antagonists are effective and safe for Crohn's disease: a meta-analysis. World J Gastroentero/ 2015;21:4744-9.

836 Moćko P, Kawalec P, Smela-Lipińska B, et al. Effectiveness and safety of vedolizumab for treatment of Crohn's disease: a systematic review and metaanalysis. Arch Med Sci 2016;12:1088-96.

837 Bonovas S, Fiorino G, Allocca M, et al. Biologic therapies and risk of infection and malignancy in patients with inflammatory bowel disease: a systematic review and network meta-analysis. Clin Gastroenterol Hepatol 2016;14:1385-97.
838 Wyant T, Leach T, Sankoh S, et al. Vedolizumab affects antibody responses to immunisation selectively in the gastrointestinal tract: randomised controlled trial results. Gut 2015;64:77-83.

839 Zeissig S, Rosati E, Dowds CM, et al. Vedolizumab is associated with changes in innate rather than adaptive immunity in patients with inflammatory bowel disease. Gut 2019;68:25-39.

840 Sands BE, Cohen RD, Isaacs KL, et al. Sa1123 Infusion-related reactions with vedolizumab treatment in patients with UC or CD during the GEMINI 1 and GEMINI 2 clinical trials. Gastroenterology 2015;148:S-232-0.

841 Papp K, Gottlieb AB, Naldi L, et al. Safety surveillance for ustekinumab and other psoriasis treatments from the Psoriasis Longitudinal Assessment and Registry (PSOLAR). J Drugs Dermato/ 2015;14:706-14.

842 López-Ferrer A, Laiz A, Puig L. The safety of ustekinumab for the treatment of psoriatic arthritis. Expert Opin Drug Saf 2017;16:733-42.

843 Tadbiri S, Peyrin-Biroulet L, Serrero M, et al. Impact of vedolizumab therapy on extra-intestinal manifestations in patients with inflammatory bowel disease: a multicentre cohort study nested in the OBSERV-IBD cohort. Aliment Pharmacol The 2018;47:485-93.

844 Cosnes J. What should be done in inflammatory bowel disease patients with prior malignancy? Dig Dis 2017;35:50-5.

845 Axelrad J, Bernheim 0, Colombel JF, et al. Risk of new or recurrent cancer in patients with inflammatory bowel disease and previous cancer exposed to immunosuppressive and anti-tumor necrosis factor agents. Clin Gastroenterol Hepatol 2016;14:58-64.

846 Annese V, Beaugerie L, Egan L, et al. European evidence-based consensus: inflammatory bowel disease and malignancies. J Crohns Colitis 2015;9:945-65.

847 Treton X, Bouhnik Y, Mary JY, et al. Azathioprine withdrawal in patients with Crohn's disease maintained on prolonged remission: a high risk of relapse. Clin Gastroenterol Hepatol 2009;7:80-5.

848 Beaugerie L, Brousse N, Bouvier AM, et al. Lymphoproliferative disorders in patients receiving thiopurines for inflammatory bowel disease: a prospective observational cohort study. Lancet 2009;374:1617-25.

849 Kennedy NA, Warner B, Johnston EL, et al. Relapse after withdrawal from anti-TNF therapy for inflammatory bowel disease: an observational study, plus systematic review and meta-analysis. Aliment Pharmacol Ther 2016:43:910-23.

850 Gisbert JP, Marín AC, Chaparro M. Systematic review: factors associated with relapse of inflammatory bowel disease after discontinuation of anti-TNF therapy. Aliment Pharmacol Ther 2015;42:391-405.

851 Rimola J, Alfaro I, Fernández-Clotet A, et al. Persistent damage on magnetic resonance enterography in patients with Crohn's disease in endoscopic remission. Aliment Pharmacol Ther 2018:48:1232-41.

852 Steinhart AH. Exit strategies for biologic therapy in IBD: is it too soon to stop? Inflamm Bowel Dis 2018;24:669-70.

853 Louis E. Stopping biologics in IBD: what is the evidence? Inflamm Bowel Dis 2018:24:725-31.

854 Louis E, Mary JY, Vernier-Massouille G, et al. Maintenance of remission among patients with Crohn's disease on antimetabolite therapy after infliximab therapy is stopped. Gastroenterology 2012;142:63-70.

855 Molander P, Färkkilä M, Ristimäki A, et al. Does fecal calprotectin predict shortterm relapse after stopping TNF $\alpha$-blocking agents in inflammatory bowel disease patients in deep remission? J Crohns Colitis 2015;9:33-40.

856 de Suray N, Salleron J, Vernier-Massouille G, et al. 864 Close monitoring of CRP and fecal calprotectin is able to predict clinical relapse in patients with crohn's disease in remission after infliximab withdrawal. a sub-analysis of the stori study. Gastroenterology 2012;142:S-149.

857 Cassinotti A, Massari A, Carmagnola S, et al. P473 How to monitor the withdrawal of maintenance treatment with azathioprine in IBD patients with deep remission: results from a prospective study on multiple non invasive tests. J Crohns Colitis 2017;11(Suppl 1):S319-S320.

858 Steinhart AH, Ewe K, Griffiths AM, et al. Corticosteroids for maintenance of remission in Crohn's disease. Cochrane Database Syst Rev 2003:CD000301.

859 Lewis JD, Scott Fl, Brensinger CM, et al. Increased mortality rates with prolonged corticosteroid therapy when compared with antitumor necrosis factor- $\alpha$-directed therapy for inflammatory bowel disease. Am J Gastroenterol 2018;113:405-17.

860 Lee N, Radford-Smith G, Taaffe DR. Bone loss in Crohn's disease: exercise as a potential countermeasure. Inflamm Bowel Dis 2005;11:1108-18.

861 Piodi LP, Poloni A, Ulivieri FM. Managing osteoporosis in ulcerative colitis: something new? World J Gastroentero/ 2014;20:14087-98.

862 Agrawal M, Arora S, Li J, et al. Bone, inflammation, and inflammatory bowe disease. Curr Osteoporos Rep 2011;9:251-7.

863 Harpavat M, Keljo DJ, Regueiro MD. Metabolic bone disease in inflammatory bowel disease. J Clin Gastroentero/ 2004;38:218-24.

864 Targownik LE, Bernstein CN, Leslie WD. Risk factors and management of osteoporosis in inflammatory bowel disease. Curr Opin Gastroenterol 2014;30:168-74. 
865 Briot K, Abitbol V, Roux C. Os et intestin. Prise en charge des ostéopathies associées aux pathologies intestinales. Revue du Rhumatisme Monographies 2016;83:261-5

866 Briot K, Geusens P, Em Bultink I, et al. Inflammatory diseases and bone fragility. Osteoporos Int 2017;28:3301-14.

867 Chatu S, Chhaya V, Holmes R, et al. Factors associated with vitamin D deficiency in a multicultural inflammatory bowel disease cohort. Frontline Gastroenterol 2013;4:51-6.

868 Frigstad SO, Høivik M, Jahnsen J, et al. Vitamin D deficiency in inflammatory bowel disease: prevalence and predictors in a Norwegian outpatient population. Scand J Gastroenterol 2017;52:100-6.

869 Bischoff-Ferrari HA, Willett WC, Wong JB, et al. Fracture prevention with vitamin D supplementation: a meta-analysis of randomized controlled trials. JAMA 2005;293:2257-64.

870 Bakker SF, Dik VK, Witte BI, et al. Increase in bone mineral density in strictly treated Crohn's disease patients with concomitant calcium and vitamin D supplementation. J Crohns Colitis 2013;7:377-84.

871 Homik J, Suarez-Almazor ME, Shea B, et al. Calcium and vitamin D for corticosteroid-induced osteoporosis. Cochrane Database Syst Rev 2000:CD000952.

872 Michaëlsson K, Melhus H, Warensjö Lemming E, et al. Long term calcium intake and rates of all cause and cardiovascular mortality: community based prospective longitudinal cohort study. BMJ 2013;346:f228.

873 van Staa TP, Leufkens HG, Cooper C. The epidemiology of corticosteroid-induced osteoporosis: a meta-analysis. Osteoporos Int 2002;13:777-87.

874 Van Staa TP, Leufkens HG, Abenhaim L, et al. Use of oral corticosteroids and risk of fractures. J Bone Miner Res 2000;15:993-1000.

875 Liu D, Ahmet A, Ward L, et al. A practical guide to the monitoring and management of the complications of systemic corticosteroid therapy. Allergy Asthma Clin Immunol 2013:9:30.

876 Kanis JA, Johansson H, Oden A, et al. Guidance for the adjustment of FRAX according to the dose of glucocorticoids. Osteoporos Int 2011;22:809-16.

877 Compston J, Cooper A, Cooper C, et al. UK clinical guideline for the prevention and treatment of osteoporosis. Arch Osteoporos 2017;12:43.

878 Buckley L, Guyatt G, Fink HA, et al. 2017 American College of Rheumatology Guideline for the Prevention and Treatment of Glucocorticoid-Induced Osteoporosis. Arthritis Rheumatol 2017;69:1521-37.

879 Reimondo G, Bovio S, Allasino B, et al. Secondary hypoadrenalism. Pituitary 2008; 11:147-54.

880 Dinsen $\mathrm{S}$, Baslund B, Klose $\mathrm{M}$, et al. Why glucocorticoid withdrawal may sometimes be as dangerous as the treatment itself. Eur J Intern Med 2013;24:714-20.

881 Green H, Paul M, Vidal L, et al. Prophylaxis of Pneumocystis pneumonia in immunocompromised non-HIV-infected patients: systematic review and metaanalysis of randomized controlled trials. Mayo Clin Proc 2007;82:1052-9.

882 Cotter TG, Gathaiya N, Catania J, et al. Low risk of pneumonia from Pneumocystis jirovecii infection in patients with inflammatory bowel disease receiving immune suppression. Clin Gastroenterol Hepatol 2017;15:850-6.

883 Tilg H, Moschen AR. Food, immunity, and the microbiome. Gastroenterology 2015; 148:1107-19.

884 Shoda R, Matsueda K, Yamato S, et al. Epidemiologic analysis of Crohn disease in Japan: increased dietary intake of $n-6$ polyunsaturated fatty acids and animal protein relates to the increased incidence of Crohn disease in Japan. Am J Clin Nutr 1996:63:741-5.

885 Jantchou P, Morois S, Clavel-Chapelon F, et al. Animal protein intake and risk of inflammatory bowel disease: The E3N prospective study. Am J Gastroenterol 2010;105:2195-201.

886 Merga Y, Campbell BJ, Rhodes JM. Mucosal barrier, bacteria and inflammatory bowel disease: possibilities for therapy. Dig Dis 2014;32:475-83.

887 Racine A, Carbonnel F, Chan SS, et al. Dietary patterns and risk of inflammatory bowel disease in Europe: results from the EPIC study. Inflamm Bowel Dis 2016;22:345-54.

888 Pironi L, Arends J, Bozzetti F, et al. ESPEN guidelines on chronic intestinal failure in adults. Clin Nutr 2016;35:247-307.

889 Vagianos K, Bector S, McConnell J, et al. Nutrition assessment of patients with inflammatory bowel disease. JPEN J Parenter Enteral Nutr 2007;31:311-9.

890 Singh S, Dulai PS, Zarrinpar A, et al. Obesity in IBD: epidemiology, pathogenesis, disease course and treatment outcomes. Nat Rev Gastroenterol Hepatol 2017; 14:110-21.

891 Flores A, Burstein E, Cipher DJ, et al. Obesity in inflammatory bowel disease: a marker of less severe disease. Dig Dis Sci 2015;60:2436-45.

892 Mueller C, Compher C, Ellen DM, et al. A.S.P.E.N. clinical guidelines: Nutrition screening, assessment, and intervention in adults. JPEN J Parenter Enteral Nutr 2011;35:16-24.

893 Sandhu A, Mosli M, Yan B, et al. Self-screening for malnutrition risk in outpatien inflammatory bowel disease patients using the Malnutrition Universal Screening Tool (MUST). JPEN J Parenter Enteral Nutr 2016;40:507-10.
894 Pouliot MC, Després JP, Lemieux S, et al. Waist circumference and abdominal sagittal diameter: best simple anthropometric indexes of abdominal visceral adipose tissue accumulation and related cardiovascular risk in men and women. Am J Cardio/ 1994;73:460-8

895 Sandall AM, Wall CL, Lomer MCE. Nutrition assessment in Crohn's disease using anthropometric, biochemical, and dietary indexes: A narrative review. J Acad Nutr Diet 2019. doi: 10.1016/j.jand.2019.04.013. [Epub ahead of print 24 Jun 2019].

896 Duncan A, Talwar D, McMillan DC, et al. Quantitative data on the magnitude of the systemic inflammatory response and its effect on micronutrient status based on plasma measurements. Am J Clin Nutr 2012;95:64-71.

897 Geerling BJ, Badart-Smook A, Stockbrügger RW, et al. Comprehensive nutritional status in recently diagnosed patients with inflammatory bowel disease compared with population controls. Eur J Clin Nutr 2000;54:514-21.

898 Ryzen E, Servis KL, DeRusso P, et al. Determination of intracellular free magnesium by nuclear magnetic resonance in human magnesium deficiency. J Am Coll Nutr 1989;8:580-7.

899 Dignass AU, Gasche C, Bettenworth D, et al. European consensus on the diagnosis and management of iron deficiency and anaemia in inflammatory bowel diseases. $J$ Crohns Colitis 2015:9:211-22.

900 Lu C, Yang J, Yu W, et al. Association between 25(OH)D level, ultraviolet exposure, geographical location, and inflammatory bowel disease activity: a systematic review and meta-analysis. PLoS One 2015;10:e0132036.

901 Del Pinto R, Pietropaoli D, Chandar AK, et al. Association between inflammatory bowel disease and vitamin $D$ deficiency: a systematic review and meta-analysis. Inflamm Bowel Dis 2015;21:2708-17.

902 Garg M, Lubel JS, Sparrow MP, et al. Review article: vitamin D and inflammatory bowel disease-established concepts and future directions. Aliment Pharmacol Ther 2012;36:324-44.

903 O'Sullivan M. Vitamin D as a novel therapy in inflammatory bowel disease: new hope or false dawn? Proc Nutr Soc 2015:74:5-12.

904 Winter RW, Collins E, Cao B, et al. Higher 25-hydroxyvitamin D levels are associated with greater odds of remission with anti-tumour necrosis factor- $\alpha$ medications among patients with inflammatory bowel diseases. Aliment Pharmacol Ther 2017:45:653-9.

905 Zator ZA, Cantu SM, Konijeti GG, et al. Pretreatment 25-hydroxyvitamin D levels and durability of anti-tumor necrosis factor- $\alpha$ therapy in inflammatory bowel diseases. JPEN J Parenter Enteral Nutr 2014;38:385-91.

906 Santos-Antunes J, Nunes AC, Lopes S, et al. The relevance of vitamin D and antinuclear antibodies in patients with inflammatory bowel disease under anti-TNF treatment: a prospective study. Inflamm Bowel Dis 2016:22:1101-6.

907 Jørgensen SP, Agnholt J, Glerup H, et al. Clinical trial: vitamin D3 treatment in Crohn's disease - a randomized double-blind placebo-controlled study. Aliment Pharmacol Ther 2010;32:377-83.

908 Miheller P, Muzes G, Hritz I, et al. Comparison of the effects of 1,25 dihydroxyvitamin $D$ and 25 hydroxyvitamin $D$ on bone pathology and disease activity in Crohn's disease patients. Inflamm Bowel Dis 2009;15:1656-62.

909 Yang L, Weaver V, Smith JP, et al. Therapeutic effect of vitamin d supplementation in a pilot study of Crohn's patients. Clin Trans/ Gastroenterol 2013;4:e33.

910 Halpin SJ, Ford AC. Prevalence of symptoms meeting criteria for irritable bowe syndrome in inflammatory bowel disease: systematic review and meta-analysis. Am J Gastroentero/ 2012;107:1474-82.

911 Prince A, Whelan K, Moosa A, et al. Nutritional problems in inflammatory bowel disease: the patient perspective. J Crohns Colitis 2011:5:443-50.

912 Prince AC, Myers CE, Joyce T, et al. Fermentable carbohydrate restriction (low FODMAP diet) in clinical practice improves functional gastrointestinal symptoms in patients with inflammatory bowel disease. Inflamm Bowel Dis 2016;22:1129-36.

913 Cox SR, Prince AC, Myers CE, et al. Fermentable carbohydrates [FODMAPs] exacerbate functional gastrointestinal symptoms in patients with inflammatory bowel disease: a randomised, double-blind, placebo-controlled, cross-over, rechallenge trial. J Crohns Colitis 2017:11:1420-9.

914 Pedersen N, Ankersen DV, Felding M, et al. Low-FODMAP diet reduces irritable bowel symptoms in patients with inflammatory bowel disease. World J Gastroenterol 2017;23:3356-66.

915 Bouguen G, Peyrin-Biroulet L. Surgery for adult Crohn's disease: what is the actual risk? Gut 2011;60:1178-81.

916 Bernstein CN, Nugent Z, Targownik LE, et al. Predictors and risks for death in a population-based study of persons with IBD in Manitoba. Gut 2015:64:1403-11.

917 Bryant RV, Trott MJ, Bartholomeusz FD, et al. Systematic review: body composition in adults with inflammatory bowel disease. Aliment Pharmacol Ther 2013;38:213-25.

918 Weisshof R, Chermesh I. Micronutrient deficiencies in inflammatory bowel disease. Curr Opin Clin Nutr Metab Care 2015;18:576-81. 
919 Lindor KD, Fleming CR, Ilstrup DM. Preoperative nutritional status and other factors that influence surgical outcome in patients with Crohn's disease. Mayo Clin Proc 1985;60:393-6.

920 Sungurtekin $\mathrm{H}$, Sungurtekin $\mathrm{U}$, Balci C, et al. The influence of nutritional status on complications after major intraabdominal surgery. J Am Coll Nutr 2004;23:227-32.

921 Alves A, Panis Y, Bouhnik Y, et al. Risk factors for intra-abdominal septic complications after a first ileocecal resection for Crohn's disease: a multivariate analysis in 161 consecutive patients. Dis Colon Rectum 2007;50:331-6.

922 Burden S, Todd C, Hill J, et al. Pre-operative nutrition support in patients undergoing gastrointestinal surgery. Cochrane Database Syst Rev 2012;11:CD008879.

923 Grass F, Pache B, Martin D, et al. Preoperative nutritional conditioning of Crohn's patients-systematic review of current evidence and practice. Nutrients 2017;9:562.

924 Weimann A, Braga M, Carli F, et al. ESPEN guideline: Clinical nutrition in surgery. Clin Nutr 2017:36:623-50.

925 Morar PS, Hodgkinson JD, Thalayasingam S, et al. Determining predictors for intraabdominal septic complications following ileocolonic resection for Crohn's disease: considerations in pre-operative and peri-operative optimisation techniques to improve outcome. J Crohns Colitis 2015;9:483-91.

926 Zerbib P, Koriche D, Truant S, et al. Pre-operative management is associated with low rate of post-operative morbidity in penetrating Crohn's disease. Aliment Pharmacol Ther 2010;32:459-65.

927 Jacobson S. Early postoperative complications in patients with Crohn's disease given and not given preoperative total parenteral nutrition. Scand J Gastroenterol 2012;47:170-7

928 Kim KJ, Han BJ, Yang SK, et al. Risk factors and outcome of acute severe lower gastrointestinal bleeding in Crohn's disease. Dig Liver Dis 2012;44:723-8.

929 Li Y, Stocchi L, Rui Y, et al. Perioperative blood transfusion and postoperative outcome in patients with Crohn's disease undergoing primary ileocolonic resection in the "biological era". J Gastrointest Surg 2015;19:1842-51.

930 Spinelli A, Allocca M, Jovani M, et al. Review article: optimal preparation for surgery in Crohn's disease. Aliment Pharmacol Ther 2014;40:1009-22.

931 Subramanian V, Saxena S, Kang JY, et al. Preoperative steroid use and risk of postoperative complications in patients with inflammatory bowel disease undergoing abdominal surgery. Am J Gastroenterol 2008;103:2373-81.

932 Ahmed Ali U, Martin ST, Rao AD, et al. Impact of preoperative immunosuppressive agents on postoperative outcomes in Crohn's disease. Dis Colon Rectum 2014; 57:663-74.

933 Aberra FN, Lewis JD, Hass D, et al. Corticosteroids and immunomodulators: postoperative infectious complication risk in inflammatory bowel disease patients. Gastroenterology 2003;125:320-7.

934 Zittan E, Milgrom R, Ma GW, et al. Preoperative anti-tumor necrosis factor therapy in patients with ulcerative colitis is not associated with an increased risk of infectious and noninfectious complications after ileal pouch-anal anastomosis. Inflamm Bowel Dis 2016;22:2442-7.

935 Balachandran R, Tøttrup A. Safety of proctocolectomy for ulcerative colitis under elective and non-elective circumstances: preoperative corticosteroid treatment worsens outcome. Dig Surg 2015;32:251-7.

936 Zaghiyan K, Melmed GY, Berel D, et al. A prospective, randomized, noninferiority trial of steroid dosing after major colorectal surgery. Ann Surg 2014;259:32-7.

937 Aytac E, Londono JM, Erem HH, et al. Impact of stress dose steroids on the outcomes of restorative proctocolectomy in patients with ulcerative colitis. Dis Colon Rectum 2013:56:1253-8.

938 Lightner AL, Shen B. Perioperative use of immunosuppressive medications in patients with Crohn's disease in the new "biological era". Gastroenterol Rep 2017;5:165-77

939 Marik PE, Varon J. Requirement of perioperative stress doses of corticosteroids: a systematic review of the literature. Arch Surg 2008;143:1222-6.

940 Myrelid P, Olaison G, Sjödahl R, et al. Thiopurine therapy is associated with postoperative intra-abdominal septic complications in abdominal surgery for Crohn's disease. Dis Colon Rectum 2009;52:1387-94.

941 Subramanian V, Pollok RC, Kang JY, et al. Systematic review of postoperative complications in patients with inflammatory bowel disease treated with immunomodulators. Br J Surg 2006;93:793-9.

942 Waterland P, Athanasiou T, Patel H. Post-operative abdominal complications in Crohn's disease in the biological era: Systematic review and meta-analysis. World J Gastrointest Surg 2016:8:274-83.

943 Kopylov U, Ben-Horin S, Zmora O, et al. Anti-tumor necrosis factor and postoperative complications in Crohn's disease: systematic review and metaanalysis. Inflamm Bowel Dis 2012;18:2404-13.

944 Narula N, Charleton D, Marshall JK. Meta-analysis: peri-operative anti-TNF $\alpha$ treatment and post-operative complications in patients with inflammatory bowel disease. Aliment Pharmacol Ther 2013;37:1057-64.
945 Yang ZP, Hong L, Wu Q, et al. Preoperative infliximab use and postoperative complications in Crohn's disease: a systematic review and meta-analysis. Int J Surg 2014;12:224-30.

946 Rosenfeld G, Qian H, Bressler B. The risks of post-operative complications following pre-operative infliximab therapy for Crohn's disease in patients undergoing abdominal surgery: a systematic review and meta-analysis. J Crohns Colitis 2013;7:868-77.

947 Huang W, Tang Y, Nong L, et al. Risk factors for postoperative intra-abdominal septic complications after surgery in Crohn's disease: A meta-analysis of observational studies. J Crohns Colitis 2015;9:293-301.

948 Lau C, Dubinsky M, Melmed G, et al. The impact of preoperative serum anti-TNF $\alpha$ therapy levels on early postoperative outcomes in inflammatory bowel disease surgery. Ann Surg 2015;261:487-96.

949 Yang Z, Wu Q, Wang F, et al. Meta-analysis: effect of preoperative infliximab use on early postoperative complications in patients with ulcerative colitis undergoing abdominal surgery. Aliment Pharmacol Ther 2012;36:922-8.

950 Zmora 0, Khaikin M, Pishori T, et al. Should ileoanal pouch surgery be staged for patients with mucosal ulcerative colitis on immunosuppressives? Int I Colorectal Dis 2007;22:289-92.

$951 \mathrm{Gu}$ J, Remzi FH, Shen B, et al. Operative strategy modifies risk of pouch-related outcomes in patients with ulcerative colitis on preoperative anti-tumor necrosis factor- $\alpha$ therapy. Dis Colon Rectum 2013;56:1243-52.

952 Menees SB, Powell C, Kurlander J, et al. A meta-analysis of the utility of C-reactive protein, erythrocyte sedimentation rate, fecal calprotectin, and fecal lactoferrin to exclude inflammatory bowel disease in adults with IBS. Am J Gastroenterol 2015:110:444-54

953 Kennedy NA, Clark A, Walkden A, et al. Clinical utility and diagnostic accuracy of faecal calprotectin for IBD at first presentation to gastroenterology services in adults aged 16-50 years. J Crohns Colitis 2015;9:41-9.

954 Walker GJ, Moore L, Heerasing N, et al. Faecal calprotectin effectively excludes inflammatory bowel disease in 789 symptomatic young adults with/without alarm symptoms: a prospective UK primary care cohort study. Aliment Pharmacol Ther 2018;47:1103-16.

955 Pavlidis P, Chedgy FJ, Tibble JA. Diagnostic accuracy and clinical application of faecal calprotectin in adult patients presenting with gastrointestinal symptoms in primary care. Scand J Gastroentero/ 2013;48:1048-54.

956 Turvill J, O'Connell S, Brooks A, et al. Evaluation of a faecal calprotectin care pathway for use in primary care. Prim Health Care Res Dev 2016;17:428-36.

957 Yang Z, Clark N, Park KT. Effectiveness and cost-effectiveness of measuring fecal calprotectin in diagnosis of inflammatory bowel disease in adults and children. Clin Gastroenterol Hepatol 2014;12:253-62.

958 Caviglia GP, Pantaleoni S, Touscoz GA, et al. Fecal calprotectin is an effective diagnostic tool that differentiates inflammatory from functional intestinal disorders. Scand J Gastroenterol 2014;49:1419-24.

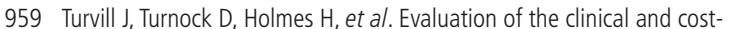
effectiveness of the York Faecal Calprotectin Care Pathway. Frontline Gastroenterol 2018:9:285-94.

960 Turvill J, Aghahoseini A, Sivarajasingham N, et al. Faecal calprotectin in patients with suspected colorectal cancer: a diagnostic accuracy study. Br J Gen Pract 2016;66:e499-506.

961 Tibble J, Sigthorsson G, Foster R, et al. Faecal calprotectin and faecal occult blood tests in the diagnosis of colorectal carcinoma and adenoma. Gut 2001:49:402-8.

962 National Institute for Health and Care Excellence: NICE guideline [NG12] Suspected cancer: recognition and referral. https://www.nice.org.uk/guidance/ng12 [Accessed 16 Dec 2018].

963 Stapley SA, Rubin GP, Alsina D, et al. Clinical features of bowel disease in patients aged $<50$ years in primary care: a large case-control study. Br J Gen Pract 2017:67:e336-44.

964 Sipponen T, Kolho KL. Fecal calprotectin in diagnosis and clinical assessment of inflammatory bowel disease. Scand J Gastroenterol 2015;50:74-80.

965 Schoepfer AM, Beglinger C, Straumann A, et al. Ulcerative colitis: correlation of the Rachmilewitz endoscopic activity index with fecal calprotectin, clinical activity, C-reactive protein, and blood leukocytes. Inflamm Bowel Dis 2009;15:1851-8.

966 Sipponen T, Kärkkäinen P, Savilahti E, et al. Correlation of faecal calprotectin and lactoferrin with an endoscopic score for Crohn's disease and histological findings. Aliment Pharmacol Ther 2008;28:1221-9.

967 Rokkas T, Portincasa P, Koutroubakis IE. Fecal calprotectin in assessing inflammatory bowel disease endoscopic activity: a diagnostic accuracy metaanalysis. J Gastrointestin Liver Dis 2018;27:299-306.

968 Lin JF, Chen JM, Zuo JH, et al. Meta-analysis: fecal calprotectin for assessment of inflammatory bowel disease activity. Inflamm Bowel Dis 2014;20:1407-15.

969 Baert F, Moortgat L, Van Assche G, et al. Mucosal healing predicts sustained clinical remission in patients with early-stage Crohn's disease. Gastroenterology 2010;138:463-8. 
970 Schnitzler F, Fidder H, Ferrante M, et al. Mucosal healing predicts long-term outcome of maintenance therapy with infliximab in Crohn's disease. Inflamm Bowel Dis 2009;15:1295-301.

971 Mosli MH, Zou G, Garg SK, et al. C-reactive protein, fecal calprotectin, and stool lactoferrin for detection of endoscopic activity in symptomatic inflammatory bowel disease patients: a systematic review and meta-analysis. Am I Gastroenterol 2015;110:802-19.

972 Yamaguchi S, Takeuchi Y, Arai K, et al. Fecal calprotectin is a clinically relevant biomarker of mucosal healing in patients with quiescent ulcerative colitis. J Gastroenterol Hepatol 2016;31:93-8.

973 Guardiola J, Lobatón T, Rodríguez-Alonso L, et al. Fecal level of calprotectin identifies histologic inflammation in patients with ulcerative colitis in clinical and endoscopic remission. Clin Gastroenterol Hepatol 2014;12:1865-70.

974 Mooiweer E, Severs M, Schipper ME, et al. Low fecal calprotectin predicts sustained clinical remission in inflammatory bowel disease patients: a plea for deep remission. J Crohns Colitis 2015;9:50-5.

975 Louis E. Fecal calprotectin: towards a standardized use for inflammatory bowe disease management in routine practice. J Crohns Colitis 2015;9:1-3

976 Zhulina Y, Cao Y, Amcoff K, et al. The prognostic significance of faecal calprotectin in patients with inactive inflammatory bowel disease. Aliment Pharmacol Ther 2016;44:495-504

977 Lasson A, Stotzer PO, Öhman L, et al. The intra-individual variability of faecal calprotectin: a prospective study in patients with active ulcerative colitis. J Crohns Colitis 2015:9:26-32.

978 Haisma SM, van Rheenen PF, Wagenmakers L, et al. Calprotectin instability may lead to undertreatment in children with IBD. Arch Dis Child 2019. doi: 10.1136/ archdischild-2018-316584. [Epub ahead of print 17 Jan 2019].

979 Williams C, Teague R. Colonoscopy. Gut 1973;14:990-1003.

980 Present DH. Toxic megacolon. Med Clin North Am 1993;77:1129-48.

981 Schwesinger WH, Levine BA, Ramos R. Complications in colonoscopy. Surg Gynecol Obstet 1979;148:270-81.

982 Waye JD. The role of colonoscopy in the differential diagnosis of inflammatory bowel disease. Gastrointest Endosc 1977;23:150-4.

983 Menees S, Higgins P, Korsnes S, et al. Does colonoscopy cause increased ulcerative colitis symptoms? Inflamm Bowel Dis 2007:13:12-18.

984 Terheggen G, Lanyi B, Schanz S, et al. Safety, feasibility, and tolerability of ileocolonoscopy in inflammatory bowel disease. Endoscopy 2008;40:656-63.

985 Froehlich F, Wietlisbach V, Gonvers J-J, et al. Impact of colonic cleansing on quality and diagnostic yield of colonoscopy: the European Panel of Appropriateness of Gastrointestinal Endoscopy European multicenter study. Gastrointest Endosc 2005;61:378-84.

986 Bessissow T, Van Keerberghen CA, Van Oudenhove L, et al. Anxiety is associated with impaired tolerance of colonoscopy preparation in inflammatory bowel disease and controls. J Crohns Colitis 2013;7:e580-7.

987 Denters MJ, Schreuder M, Depla AC, et al. Patients' perception of colonoscopy: patients with inflammatory bowel disease and irritable bowel syndrome experience the largest burden. Eur I Gastroenterol Hepatol 2013;25:964-72.

988 Berkelhammer C, Ekambaram A, Silva RG, et al. Low-volume oral colonoscopy bowel preparation: sodium phosphate and magnesium citrate. Gastrointest Endosc 2002:56:89-94.

989 Restellini S, Kherad O, Bessissow T, et al. Systematic review and meta-analysis of colon cleansing preparations in patients with inflammatory bowel disease. World $J$ Gastroenterol 2017;23:5994-6002.

990 Farmer KC. Methods for measuring and monitoring medication regimen adherence in clinical trials and clinical practice. Clin Ther 1999;21:1074-90.

991 Jackson CA, Clatworthy J, Robinson A, et al. Factors associated with non-adherence to oral medication for inflammatory bowel disease: a systematic review. Am J Gastroenterol 2010;105:525-39.

992 Selinger CP, Eaden J, Jones DB, et al. Modifiable factors associated with nonadherence to maintenance medication for inflammatory bowel disease. Inflamm Bowel Dis 2013;19:2199-206.

993 Goodhand JR, Kamperidis N, Sirwan B, et al. Factors associated with thiopurine non-adherence in patients with inflammatory bowel disease. Aliment Pharmacol Ther 2013:38:1097-108.

994 Ediger JP, Walker JR, Graff L, et al. Predictors of medication adherence in inflammatory bowel disease. Am J Gastroenterol 2007;102:1417-26.

995 Lopez A, Billioud V, Peyrin-Biroulet C, et al. Adherence to anti-TNF therapy in inflammatory bowel diseases: a systematic review. Inflamm Bowel Dis 2013;19:1528-33.

996 Kane S, Huo D, Aikens J, et al. Medication nonadherence and the outcomes of patients with quiescent ulcerative colitis. Am J Med 2003;114:39-43.

997 van der Have M, Oldenburg B, Kaptein AA, et al. Non-adherence to anti-TNF therapy is associated with illness perceptions and clinical outcomes in outpatients with inflammatory bowel disease: results from a prospective multicentre study. J Crohns Colitis 2016;10:549-55.
998 Vangeli E, Bakhshi S, Baker A, et al. A systematic review of factors associated with non-adherence to treatment for immune-mediated inflammatory diseases. Adv Ther 2015;32:983-1028.

999 Gray WN, Denson LA, Baldassano RN, et al. Treatment adherence in adolescents with inflammatory bowel disease: the collective impact of barriers to adherence and anxiety/depressive symptoms. J Pediatr Psychol 2012;37:282-91.

1000 Waters BM, Jensen L, Fedorak RN. Effects of formal education for patients with inflammatory bowel disease: a randomized controlled trial. Can I Gastroenterol 2005;19:235-44.

1001 Depont F, Berenbaum F, Filippi J, et al. Interventions to improve adherence in patients with immune-mediated inflammatory disorders: a systematic review. PLoS One 2015;10:e0145076.

1002 Hawthorne AB, Rubin G, Ghosh S. Review article: medication non-adherence in ulcerative colitis-strategies to improve adherence with mesalazine and other maintenance therapies. Aliment Pharmacol Ther 2008;27:1157-66.

1003 Moshkovska T, Stone MA, Smith RM, et al. Impact of a tailored patient preference intervention in adherence to 5 -aminosalicylic acid medication in ulcerative colitis: results from an exploratory randomized controlled trial. Inflamm Bowel Dis 2011;17:1874-81.

1004 Elkjaer M, Shuhaibar M, Burisch J, et al. E-health empowers patients with ulcerative colitis: a randomised controlled trial of the web-guided 'Constant-care' approach Gut 2010:59:1652-61.

1005 Tabibian A, Tabibian JH, Beckman LJ, et al. Predictors of health-related quality of life and adherence in Crohn's disease and ulcerative colitis: implications for clinical management. Dig Dis Sci 2015;60:1366-74.

1006 National Institute for Health and Care Excellence. Quality standard [QS43] Smoking: supporting people to stop. https://www.nice.org.uk/guidance/qs43 (Accessed 15 Dec 2018).

1007 Johnson GJ, Cosnes J, Mansfield JC. Review article: smoking cessation as primary therapy to modify the course of Crohn's disease. Aliment Pharmacol Ther 2005;21:921-31

1008 Bornemann P, Eissa A, Strayer SM. Smoking cessation: What should you recommend? J Fam Pract 2016;65:22-9.

1009 To N, Gracie DJ, Ford AC. Systematic review with meta-analysis: the adverse effects of tobacco smoking on the natural history of Crohn's disease. Aliment Pharmacol Ther 2016:43:549-61.

1010 Regueiro M, Kip KE, Cheung 0, et al. Cigarette smoking and age at diagnosis of inflammatory bowel disease. Inflamm Bowel Dis 2005;11:42-7.

1011 Lunney PC, Kariyawasam VC, Wang RR, et al. Smoking prevalence and its influence on disease course and surgery in Crohn's disease and ulcerative colitis. Aliment Pharmacol Ther 2015;42:61-70.

1012 Baucom RB, Poulose BK, Herline AJ, et al. Smoking as dominant risk factor for anastomotic leak after left colon resection. Am J Surg 2015;210:1-5.

1013 Sharma A, Deeb AP, lannuzzi JC, et al. Tobacco smoking and postoperative outcomes after colorectal surgery. Ann Surg 2013;258:296-300.

1014 van der Heide F, Dijkstra A, Weersma RK, et al. Effects of active and passive smoking on disease course of Crohn's disease and ulcerative colitis. Inflamm Bowel Dis 2009;15:1199-207.

1015 Seksik P, Nion-Larmurier I, Sokol H, et al. Effects of light smoking consumption on the clinical course of Crohn's disease. Inflamm Bowel Dis 2009:15:734-41.

1016 Cosnes J, Nion-Larmurier I, Afchain P, et al. Gender differences in the response of colitis to smoking. Clin Gastroenterol Hepatol 2004:2:41-8.

1017 Coward S, Heitman SJ, Clement F, et al. Funding a smoking cessation program for Crohn's disease: an economic evaluation. Am I Gastroenterol 2015:110:368-77.

1018 Saadoune N, Peyrin-Biroulet L, Baumann C, et al. Beliefs and behaviour about smoking among inflammatory bowel disease patients. Eur I Gastroenterol Hepatol 2015;27:797-803

1019 De Bie C, Ballet V, Hendriks N, et al. Smoking behaviour and knowledge of the health effects of smoking in patients with inflammatory bowel disease. Aliment Pharmacol Ther 2015:42:1294-302.

1020 Biedermann L, Fournier N, Misselwitz B, et al. HighrRates of smoking especially in female Crohn's disease patients and low use of supportive measures to achieve smoking cessation: data from the Swiss IBD Cohort Study. J Crohns Colitis 2015:9:819-29

1021 Lawrance IC, Murray K, Batman B, et al. Crohn's disease and smoking: is it ever too late to quit? J Crohns Colitis 2013;7:e665-71.

1022 Nunes T, Etchevers MJ, Merino O, et al. High smoking cessation rate in Crohn's disease patients after physician advice-the TABACROHN Study. I Crohns Colitis 2013;7:202-7

1023 Takahashi H, Matsui T, Hisabe T, et al. Second peak in the distribution of age at onset of ulcerative colitis in relation to smoking cessation. J Gastroenterol Hepatol 2014;29:1603-8.

1024 Rosenfeld G, Bressler B. The truth about cigarette smoking and the risk of inflammatory bowel disease. Am J Gastroenterol 2012;107:1407-8. 
1025 Motley RJ, Rhodes J, Kay S, et al. Late presentation of ulcerative colitis in exsmokers. Int J Colorectal Dis 1988;3:171-5.

1026 Srivasta ED, Newcombe RG, Rhodes J, et al. Smoking and ulcerative colitis: a community study. Int J Colorectal Dis 1993;8:71-4.

1027 Beaugerie L, Massot N, Carbonnel F, et al. Impact of cessation of smoking on the course of ulcerative colitis. Am J Gastroenterol 2001;96:2113-6.

1028 Joelsson M, Benoni C, Oresland T. Does smoking influence the risk of pouchitis following ileal pouch anal anastomosis for ulcerative colitis? Scand I Gastroenterol 2006;41:929-33.

1029 Calabrese E, Yanai H, Shuster D, et al. Low-dose smoking resumption in ex-smokers with refractory ulcerative colitis. J Crohns Colitis 2012;6:756-62.

1030 Pullan RD, Rhodes J, Ganesh S, et al. Transdermal nicotine for active ulcerative colitis. N Eng/ J Med 1994;330:811-5.

1031 Thomas GA, Rhodes J, Ragunath K, et al. Transdermal nicotine compared with oral prednisolone therapy for active ulcerative colitis. Eur I Gastroenterol Hepatol 1996:8:769-76.

1032 Thomas GA, Rhodes J, Mani V, et al. Transdermal nicotine as maintenance therapy for ulcerative colitis. N Engl J Med 1995;332:988-92.

1033 Lunney PC, Leong RW. Review article: Ulcerative colitis, smoking and nicotine therapy. Aliment Pharmacol Ther 2012;36:997-1008.

1034 Watson JP, Lewis RA. Ulcerative colitis responsive to smoking and to nicotine chewing gum in a patient with $\alpha 1$ anti-trypsin deficiency. Respiratory Medicine 1995;89:635-6

1035 Lashner BA, Hanauer SB, Silverstein MD. Testing nicotine gum for ulcerative colitis patients. Experience with single-patient trials. Dig Dis Sci 1990;35:827-32.

1036 Camus M, Gallois C, Gallois G, et al. Ulcerative colitis and electronic cigarette: what's the matter? Am I Gastroenterol 2014;109:608-9.

1037 Frolkis AD, Vallerand IA, Shaheen A-A, et al. Depression increases the risk of inflammatory bowel disease, which may be mitigated by the use of antidepressants in the treatment of depression. Gut 2019;68:1606-12.

1038 Walker JR, Ediger JP, Graff LA, et al. The Manitoba IBD cohort study: a populationbased study of the prevalence of lifetime and 12-month anxiety and mood disorders. Am J Gastroenterol 2008;103:1989-97.

1039 Goodhand JR, Wahed M, Mawdsley JE, et al. Mood disorders in inflammatory bowel disease: relation to diagnosis, disease activity, perceived stress, and other factors. Inflamm Bowel Dis 2012;18:2301-9.

1040 Mikocka-Walus AA, Turnbull D, Holtmann G, et al. An integrated model of care for inflammatory bowel disease sufferers in Australia: development and the effects of its implementation. Inflamm Bowel Dis 2012:18:1573-81.

1041 Gracie DJ, Williams CJ, Sood R, et al. Negative effects on psychological health and quality of life of genuine irritable bowel syndrome-type symptoms in patients with inflammatory bowel disease. Clin Gastroenterol Hepatol 2017;15:376-84.

1042 Abdalla MI, Sandler RS, Kappelman MD, et al. Prevalence and impact of inflammatory bowel disease-irritable bowel syndrome on patient-reported outcomes in CCFA Partners. Inflamm Bowel Dis 2017:23:325-31.

1043 Maunder RG, Levenstein S. The role of stress in the development and clinical course of inflammatory bowel disease: epidemiological evidence. Curr Mol Med 2008:8:247-52.

1044 Deter HC, Keller W, von Wietersheim J, et al. Psychological treatment may reduce the need for healthcare in patients with Crohn's disease. Inflamm Bowel Dis 2007:13:745-52

1045 Mizrahi MC, Reicher-Atir R, Levy S, et al. Effects of guided imagery with relaxation training on anxiety and quality of life among patients with inflammatory bowel disease. Psychol Health 2012;27:1463-79.

1046 Timmer A, Preiss JC, Motschall E, et al. Psychological interventions for treatment of inflammatory bowel disease. Cochrane Database Syst Rev 2011;2:CD006913.

1047 Thompson RD, Craig A, Crawford EA, et al. Longitudinal results of cognitive behavioral treatment for youths with inflammatory bowel disease and depressive symptoms. J Clin Psychol Med Settings 2012;19:329-37.

1048 Szigethy E, Whitton SW, Levy-Warren A, et al. Cognitive-behavioral therapy for depression in adolescents with inflammatory bowel disease: a pilot study. J Am Acad Child Adolesc Psychiatry 2004;43:1469-77.

1049 Mussell M, Böcker U, Nagel N, et al. Reducing psychological distress in patients with inflammatory bowel disease by cognitive-behavioural treatment: exploratory study of effectiveness. Scand I Gastroenterol 2003;38:755-62.

1050 Mikocka-Walus A, Bampton P, Hetzel D, et al. Cognitive-behavioural therapy for inflammatory bowel disease: 24 -month data from a randomised controlled trial. Int J Behav Med 2017;24:127-35.

1051 Neilson K, Ftanou M, Monshat K, et al. A controlled study of a group mindfulness intervention for individuals living with inflammatory bowel disease. Inflamm Bowel Dis 2016:22:694-701.

1052 Berrill JW, Sadlier M, Hood K, et al. Mindfulness-based therapy for inflammatory bowel disease patients with functional abdominal symptoms or high perceived stress levels. J Crohns Colitis 2014;8:945-55.
1053 Jedel S, Hoffman A, Merriman P, et al. A randomized controlled trial of mindfulnessbased stress reduction to prevent flare-up in patients with inactive ulcerative colitis. Digestion 2014;89:142-55.

1054 Peters SL, Muir JG, Gibson PR. Review article: gut-directed hypnotherapy in the management of irritable bowel syndrome and inflammatory bowel disease. Aliment Pharmacol Ther 2015;41:1104-15.

1055 Keefer L, Taft TH, Kiebles JL, et al. Gut-directed hypnotherapy significantly augments clinical remission in quiescent ulcerative colitis. Aliment Pharmacol Ther 2013;38:761-71

1056 Wojtowicz AA, Greenley RN, Gumidyala AP, et al. Pain severity and pain catastrophizing predict functional disability in youth with inflammatory bowel disease. J Crohns Colitis 2014;8:1118-24.

1057 Deberry JJ, Bielefeldt K, Davis BM, et al. Abdominal pain and the neurotrophic system in ulcerative colitis. Inflamm Bowel Dis 2014;20:2330-9.

1058 Schirbel A, Reichert A, Roll S, et al. Impact of pain on health-related quality of life in patients with inflammatory bowel disease. World I Gastroenterol 2010;16:3168-77.

1059 Seres G, Kovács Z, Kovács A, et al. Different associations of health related quality of life with pain, psychological distress and coping strategies in patients with irritable bowel syndrome and inflammatory bowel disorder. J Clin Psychol Med Settings 2008;15:287-95.

1060 Zeitz J, Ak M, Müller-Mottet S, et al. Pain in IBD patients: very frequent and frequently insufficiently taken into account. PLoS One 2016;11:e0156666.

1061 Boyle M, Murphy S, Leyden J, et al. Analysis of patient perception of pain and management strategies in IBD. J Crohns Colitis 2015;9(Suppl 1):S389.

1062 Coates MD, Lahoti M, Binion DG, et al. Abdominal pain in ulcerative colitis. Inflamm Bowel Dis 2013;19:2207-14.

1063 Seth N, Abdul-Baki H, Mahoney N, et al. Causes and consequences of chronic abdominal pain in crohn's disease: A five-year prospective study. Am J Gastroenterol 2014;109(Suppl 2):S490.

1064 Kinnucan J, Goeppinger S, Ackerman A, et al. P-051 YI Assessment of pain in IBD patients at a tertiary center. Inflamm Bowel Dis 2013;19(Suppl 1):S47-8.

1065 Jelsness-Jørgensen LP, Bernklev T, Moum B. Coexisting irritable bowel-like symptoms in inflammatory bowel disease in remission is associated with impaired social functioning and increased bodily pain. Gastroenterol Nurs 2014;37:280-7.

1066 Fukuba N, Ishihara S, Tada Y, et al. Prevalence of irritable bowel syndrome-like symptoms in ulcerative colitis patients with clinical and endoscopic evidence of remission: prospective multicenter study. Scand I Gastroenterol 2014;49:674-80.

1067 van Hoboken EA, Thijssen AY, Verhaaren R, et al. Symptoms in patients with ulcerative colitis in remission are associated with visceral hypersensitivity and mast cell activity. Scand I Gastroenterol 2011;46:981-7.

1068 Shen B, Sanmiguel C, Bennett AE, et al. Irritable pouch syndrome is characterized by visceral hypersensitivity. Inflamm Bowel Dis 2011;17:994-1002.

1069 Buskila D, Odes LR, Neumann L, et al. Fibromyalgia in inflammatory bowel disease. J Rheumatol 1999;26:1167-71.

1070 Gracie DJ, Irvine AJ, Sood R, et al. Effect of psychological therapy on disease activity, psychological comorbidity, and quality of life in inflammatory bowel disease: a systematic review and meta-analysis. Lancet Gastroenterol Hepatol 2017:2:189-99.

1071 Shaw L, Ehrlich A. Relaxation training as a treatment for chronic pain caused by ulcerative colitis. Pain 1987;29:287-93.

1072 Buckley JP, Kappelman MD, Allen JK, et al. The burden of comedication among patients with inflammatory bowel disease. Inflamm Bowel Dis 2013;19:2725-36.

1073 Hanson KA, Loftus EV, Harmsen WS, et al. Clinical features and outcome of patients with inflammatory bowel disease who use narcotics: a case-control study. Inflamm Bowel Dis 2009;15:772-7.

1074 Cross RK, Wilson KT, Binion DG. Narcotic use in patients with Crohn's disease. Am J Gastroenterol 2005;100:2225-9.

1075 Anderson A, Click B, Ramos-Rivers C, et al. The association between sustained poor quality of life and future opioid use in inflammatory bowel disease. Inflamm Bowel Dis 2018;24:1380-8.

1076 Cheng D, Anderson A, Ramos-Rivers C, et al. 688 Patterns between multi-year steroid use in patients with inflammatory bowel disease and quality of life, disease severity, and healthcare utilization. Am J Gastroenterol 2016;111(Suppl):S312-3.

1077 Targownik LE, Nugent Z, Singh H, et al. The prevalence and predictors of opioid use in inflammatory bowel disease: a population-based analysis. Am I Gastroenterol 2014:109:1613-20.

1078 Flath C, Motley A, Nohl A, et al. Narcotic use, psychiatric history, and corticosteroid use are associated with depressive symptoms in patients with IBD. Am J Gastroenterol 2016;111(Suppl 1):S296-7.

1079 Heppell J, Farkouh E, Dubé S, et al. Toxic megacolon. An analysis of 70 cases. Dis Colon Rectum 1986;29:789-92.

1080 Hartong WA, Arvanitakis C, Skibba RM, et al. Treatment of toxic megacolon. A comparative review of 29 patients. Am J Dig Dis 1977;22:195-200. 
1081 van Langenberg DR, Gibson PR. Systematic review: fatigue in inflammatory bowel disease. Aliment Pharmacol Ther 2010;32:131-43.

1082 Czuber-Dochan W, Norton C, Bredin F, et al. Healthcare professionals' perceptions of fatigue experienced by people with IBD. J Crohns Colitis 2014;8:835-44.

1083 Grimstad T, Norheim KB, Isaksen K, et al. Fatigue in newly diagnosed inflammatory bowel disease. J Crohns Colitis 2015;9:725-30.

1084 van Langenberg DR, Gibson PR. Factors associated with physical and cognitive fatigue in patients with Crohn's disease: a cross-sectional and longitudinal study. Inflamm Bowel Dis 2014;20:115-25.

1085 Bager P, Befrits R, Wikman 0, et al. Fatigue in out-patients with inflammatory bowel disease is common and multifactorial. Aliment Pharmacol Ther 2012;35:133-41.

1086 Römkens TE, van Vugt-van Pinxteren MW, Nagengast FM, et al. High prevalence of fatigue in inflammatory bowel disease: A case control study. J Crohns Colitis 2011;5:332-7.

1087 Graff LA, Vincent N, Walker JR, et al. A population-based study of fatigue and sleep difficulties in inflammatory bowel disease. Inflamm Bowel Dis 2011;17:1882-9.

1088 Romberg-Camps MJ, Bol Y, Dagnelie PC, et al. Fatigue and health-related quality of life in inflammatory bowel disease: results from a population-based study in the Netherlands: the IBD-South Limburg cohort. Inflamm Bowel Dis 2010;16:2137-47.

1089 Cohen BL, Zoëga H, Shah SA, et al. Fatigue is highly associated with poor healthrelated quality of life, disability and depression in newly-diagnosed patients with inflammatory bowel disease, independent of disease activity. Aliment Pharmacol Ther 2014;39:811-22.

1090 Czuber-Dochan W, Dibley LB, Terry H, et al. The experience of fatigue in people with inflammatory bowel disease: an exploratory study. J Adv Nurs 2013;69:1987-99.

1091 Vogelaar L, van't Spijker A, van Tilburg AJ, et al. Determinants of fatigue in Crohn's disease patients. Eur I Gastroenterol Hepatol 2013;25:246-51.

1092 Piche T, Ducrotté P, Sabate JM, et al. Impact of functional bowel symptoms on quality of life and fatigue in quiescent Crohn disease and irritable bowel syndrome. Neurogastroenterol Motil 2010;22:626-e174.

1093 Minderhoud IM, Oldenburg B, van Dam PS, et al. High prevalence of fatigue in quiescent inflammatory bowel disease is not related to adrenocortical insufficiency. Am J Gastroenterol 2003;98:1088-93.

1094 Danese S, Hoffman C, Vel S, et al. Anaemia from a patient perspective in inflammatory bowel disease: results from the European Federation of Crohn's and Ulcerative Colitis Association's online survey. Eur J Gastroenterol Hepatol 2014;26:1385-91.

1095 Goldenberg BA, Graff LA, Clara I, et al. Is iron deficiency in the absence of anemia associated with fatigue in inflammatory bowel disease? Am I Gastroenterol 2013:108:1392-7.

1096 Peyrin-Biroulet L, Williet N, Cacoub P. Guidelines on the diagnosis and treatment of iron deficiency across indications: a systematic review. Am I Clin Nutr 2015;102:1585-94

1097 van Langenberg DR, Della Gatta P, Warmington SA, et al. Objectively measured muscle fatigue in Crohn's disease: correlation with self-reported fatigue and associated factors for clinical application. J Crohns Colitis 2014;8:137-46.

1098 Vogelaar L, de Haar C, Aerts BR, et al. Fatigue in patients with inflammatory bowel disease is associated with distinct differences in immune parameters. Clin Exp Gastroenterol 2017:10:83-90.

1099 Lichtenstein GR, Bala M, Han C, et al. Infliximab improves quality of life in patients with Crohn's disease. Inflamm Bowel Dis 2002;8:237-43.

1100 Loftus EV, Feagan BG, Colombel JF, et al. Effects of adalimumab maintenance therapy on health-related quality of life of patients with Crohn's disease: patientreported outcomes of the CHARM trial. Am I Gastroenterol 2008;103:3132-41.

1101 Minderhoud IM, Samsom M, Oldenburg B. Crohn's disease, fatigue, and infliximab: is there a role for cytokines in the pathogenesis of fatigue? World I Gastroenterol 2007:13:2089-93

1102 Vogelaar L, van't Spijker A, Timman R, et al. Fatigue management in patients with IBD: a randomised controlled trial. Gut 2014;63:911-8.

1103 García-Vega E, Fernandez-Rodriguez C. A stress management programme for Crohn's disease. Behav Res Ther 2004:42:367-83.

1104 Harbord M, Annese V, Vavricka SR, et al. The First European Evidence-based Consensus on Extra-intestinal Manifestations in Inflammatory Bowel Disease. J Crohns Colitis 2016;10:239-54.

1105 Orchard TR, Wordsworth BP, Jewell DP. Peripheral arthropathies in inflammatory bowel disease: their articular distribution and natural history. Gut 1998;42:387-91.

1106 Sykes MP, Doll H, Sengupta R, et al. Delay to diagnosis in axial spondyloarthritis: are we improving in the UK? Rheumatology 2015;54:kev288-4.

1107 Passalent LA. Physiotherapy for ankylosing spondylitis: evidence and application. Curr Opin Rheumatol 2011;23:142-7.

1108 Braun J, Zochling J, Baraliakos X, et al. Efficacy of sulfasalazine in patients with inflammatory back pain due to undifferentiated spondyloarthritis and early ankylosing spondylitis: a multicentre randomised controlled trial. Ann Rheum Dis 2006;65:1147-53
1109 Haibel H, Sieper J. Use of methotrexate in patients with ankylosing spondylitis. Clin Exp Rheumatol 2010;28:S128-31.

1110 Bonner GF, Fakhri A, Vennamaneni SR. A long-term cohort study of nonsteroidal anti-inflammatory drug use and disease activity in outpatients with inflammatory bowel disease. Inflamm Bowel Dis 2004;10:751-7.

1111 Feagins LA. Role of transforming growth factor $\beta$ in inflammatory bowel disease and colitis-associated colon cancer. Inflamm Bowel Dis 2010;16:1963-8.

1112 Kvasnovsky CL, Aujla U, Bjarnason I. Nonsteroidal anti-inflammatory drugs and exacerbations of inflammatory bowel disease. Scand I Gastroenterol 2015;50:255-63.

1113 Ribaldone DG, Fagoonee S, Astegiano M, et al. Coxib's safety in patients with inflammatory bowel diseases: a meta-analysis. Pain Physician 2015;18:599-607.

1114 Miao XP, Li JS, Ouyang Q, et al. Tolerability of selective cyclooxygenase 2 inhibitors used for the treatment of rheumatological manifestations of inflammatory bowel disease. Cochrane Database Syst Rev 2014;10:CD007744.

1115 Ghouri YA, Richards DM, Rahimi EF, et al. Systematic review of randomized controlled trials of probiotics, prebiotics, and synbiotics in inflammatory bowel disease. Clin Exp Gastroenterol 2014;7:473-87.

1116 Mallon P, McKay D, Kirk S, et al. Probiotics for induction of remission in ulcerative colitis. Cochrane Database Syst Rev 2007:CD005573.

1117 Naidoo K, Gordon M, Fagbemi AO, et al. Probiotics for maintenance of remission in ulcerative colitis. Cochrane Database Syst Rev 2011:12:CD007443.

1118 Fujimori S, Gudis K, Mitsui K, et al. A randomized controlled trial on the efficacy of synbiotic versus probiotic or prebiotic treatment to improve the quality of life in patients with ulcerative colitis. Nutrition 2009;25:520-5.

1119 Costello SP, Hughes PA, Waters O, et al. Effect of fecal microbiota transplantation on 8-week remission in patients with ulcerative colitis: a randomized clinical trial. JAMA 2019:321:156-64.

1120 Paramsothy S, Kamm MA, Kaakoush NO, et al. Multidonor intensive faecal microbiota transplantation for active ulcerative colitis: a randomised placebocontrolled trial. Lancet 2017;389:1218-28.

1121 Moayyedi P, Surette MG, Kim PT, et al. Fecal microbiota transplantation induces remission in patients with active ulcerative colitis in a randomized controlled trial. Gastroenterology 2015;149:102-9.

1122 Rossen NG, Fuentes S, van der Spek MJ, et al. Findings from a randomized controlled trial of fecal transplantation for patients with ulcerative colitis. Gastroenterology 2015;149:110-8.

1123 El-Nachef N, Piceno YM, Kassam Z, et al. The role of fecal microbiota transplantation in ulcerative colitis and Crohn's disease: results from a parallel inflammatory bowel disease cohort study. Gastroenterology 2017;152(Suppl 1):S1008.

1124 Costello SP, Soo W, Bryant RV, et al. Systematic review with meta-analysis: faecal microbiota transplantation for the induction of remission for active ulcerative colitis. Aliment Pharmacol Ther 2017:46:213-24.

1125 Jacob V, Crawford C, Cohen-Mekelburg S, et al. Single delivery of high-diversity fecal microbiota preparation by colonoscopy Is safe and effective in increasing microbial diversity in active ulcerative colitis. Inflamm Bowel Dis 2017;23:903-11.

1126 Kelly CR, Ananthakrishnan AN. Manipulating the microbiome with fecal transplantation to treat ulcerative colitis. JAMA 2019;321:151-2.

1127 König J, Siebenhaar A, Högenauer C, et al. Consensus report: faecal microbiota transfer - clinical applications and procedures. Aliment Pharmacol Ther 2017; 45:222-39.

1128 Rawsthorne P, Clara I, Graff LA, et al. The Manitoba Inflammatory Bowel Disease Cohort Study: a prospective longitudinal evaluation of the use of complementary and alternative medicine services and products. Gut 2012;61:521-7.

1129 Gröchenig HP, Dejaco C, Eckhard G, et al. P677 Use and predictors of complementary and alternative medicine in patients with inflammatory bowel disease: Results of a multicentre study of the Austrian IBD Study Group (ATISG). J Crohns Colitis 2016;10(Suppl 1):S446-7.

1130 Lang A, Salomon N, Wu JC, et al. Curcumin in combination with mesalamine induces remission in patients with mild-to-moderate ulcerative colitis in a randomized controlled trial. Clin Gastroenterol Hepatol 2015;13:1444-9.

1131 Hanai H, lida T, Takeuchi K, et al. Curcumin maintenance therapy for ulcerative colitis: randomized, multicenter, double-blind, placebo-controlled trial. Clin Gastroenterol Hepatol 2006;4:1502-6.

1132 Moss AC. Curcumin for maintenance therapy in ulcerative colitis. Clin Gastroenterol Hepatol 2007;5:642.

1133 Feagan BG, Sandborn WJ, Mittmann U, et al. Omega-3 free fatty acids for the maintenance of remission in Crohn disease: the EPIC Randomized Controlled Trials. JAMA 2008:299:1690-7.

1134 Summers RW, Elliott DE, Urban JF, et al. Trichuris suis therapy for active ulcerative colitis: a randomized controlled trial. Gastroenterology 2005;128:825-32.

1135 Robinson RJ, Krzywicki T, Almond L, et al. Effect of a low-impact exercise program on bone mineral density in Crohn's disease: a randomized controlled trial. Gastroenterology 1998;115:36-41. 
1136 Cheifetz AS, Gianotti R, Luber R, et al. Complementary and alternative medicines used by patients with inflammatory bowel diseases. Gastroenterology 2017; 152:415-29.

1137 Weiss A, Friedenberg F. Patterns of cannabis use in patients with inflammatory bowel disease: a population based analysis. Drug Alcohol Depend 2015;156:84-9.

1138 Ahmed W, Katz S. Therapeutic use of cannabis in inflammatory bowel disease. Gastroenterol Hepatol 2016;12:668-79.

1139 Lal S, Prasad N, Ryan M, et al. Cannabis use amongst patients with inflammatory bowel disease. Eur J Gastroenterol Hepatol 2011;23:891-6.

1140 Irving PM, Iqbal T, Nwokolo C, et al. A randomized, double-blind, placebocontrolled, parallel-group, pilot study of cannabidiol-rich botanical extract in the symptomatic treatment of ulcerative colitis. Inflamm Bowel Dis 2018;24:714-24.

1141 Naftali T, Bar-Lev Schleider L, Dotan I, et al. Cannabis induces a clinical response in patients with Crohn's disease: a prospective placebo-controlled study. Clin Gastroenterol Hepatol 2013;11:1276-80.

1142 Jess T, Loftus EV, Velayos FS, et al. Risk of intestinal cancer in inflammatory bowel disease: a population-based study from olmsted county, Minnesota. Gastroenterology 2006;130:1039-46.

1143 Eaden JA, Abrams KR, Mayberry JF. The risk of colorectal cancer in ulcerative colitis: a meta-analysis. Gut 2001;48:526-35.

1144 Ekbom A, Helmick C, Zack M, et al. Ulcerative colitis and colorectal cancer. A population-based study. N Engl J Med 1990;323:1228-33.

1145 Winther KV, Jess T, Langholz E, et al. Long-term risk of cancer in ulcerative colitis: a population-based cohort study from Copenhagen County. Clin Gastroenterol Hepatol 2004;2:1088-95.

1146 Lakatos L, Mester G, Erdelyi Z, et al. Risk factors for ulcerative colitis-associated colorectal cancer in a Hungarian cohort of patients with ulcerative colitis: results of a population-based study. Inflamm Bowel Dis 2006;12:205-11.

1147 Rutter M, Saunders B, Wilkinson K, et al. Severity of inflammation is a risk factor for colorectal neoplasia in ulcerative colitis. Gastroenterology 2004;126:451-9.

1148 Gupta RB, Harpaz N, Itzkowitz S, et al. Histologic inflammation is a risk factor for progression to colorectal neoplasia in ulcerative colitis: a cohort study. Gastroenterology 2007;133:1099-105.

1149 Velayos FS, Loftus EV, Jess T, et al. Predictive and protective factors associated with colorectal cancer in ulcerative colitis: A case-control study. Gastroenterology 2006;130:1941-9

1150 Baars JE, Looman CW, Steyerberg EW, et al. The risk of inflammatory bowel disease-related colorectal carcinoma is limited: results from a nationwide nested case-control study. Am J Gastroentero/ 2011;106:319-28.

1151 Mahmoud R, Shah SC, Ten Hove JR, et al. No association between pseudopolyps and colorectal neoplasia in patients with inflammatory bowel diseases. Gastroenterology 2019;156.

1152 Sugita A, Sachar DB, Bodian C, et al. Colorectal cancer in ulcerative colitis. Influence of anatomical extent and age at onset on colitis-cancer interval. Gut 1991;32:167-9.

1153 Canavan C, Abrams KR, Mayberry J. Meta-analysis: colorectal and small bowel cancer risk in patients with Crohn's disease. Aliment Pharmacol Ther 2006;23:1097-104.

1154 Selinger CP, Andrews JM, Titman A, et al. Long-term follow-up reveals low incidence of colorectal cancer, but frequent need for resection, among Australian patients with inflammatory bowel disease. Clin Gastroenterol Hepatol 2014;12:644-50.

1155 Annese V, Daperno M, Rutter MD, et al. European evidence based consensus for endoscopy in inflammatory bowel disease. J Crohns Colitis 2013;7:982-1018.

1156 Cairns SR, Scholefield JH, Steele RJ, et al. Guidelines for colorectal cancer screening and surveillance in moderate and high risk groups (update from 2002). Gut 2010;59:666-89.

1157 Farraye FA, Odze RD, Eaden J, et al. AGA medical position statement on the diagnosis and management of colorectal neoplasia in inflammatory bowel disease. Gastroenterology 2010;138:738-45.

1158 Van Assche G, Dignass A, Bokemeyer B, et al. Second European evidence-based consensus on the diagnosis and management of ulcerative colitis part 3: special situations. J Crohns Colitis 2013;7:1-33.

1159 Collins PD, Mpofu C, Watson AJ, et al. Strategies for detecting colon cancer and/ or dysplasia in patients with inflammatory bowel disease. Cochrane Database Syst Rev 2006:CD000279.

1160 Lashner BA, Kane SV, Hanauer SB. Colon cancer surveillance in chronic ulcerative colitis: historical cohort study. Am J Gastroenterol 1990;85:1083-7.

1161 Karlén P, Kornfeld D, Broström O, et al. Is colonoscopic surveillance reducing colorectal cancer mortality in ulcerative colitis? A population based case control study. Gut 1998:42:711-4.

1162 Choi PM, Nugent FW, Schoetz DJ, et al. Colonoscopic surveillance reduces mortality from colorectal cancer in ulcerative colitis. Gastroenterology 1993;105:418-24.

1163 Lutgens MW, Oldenburg B, Siersema PD, et al. Colonoscopic surveillance improves survival after colorectal cancer diagnosis in inflammatory bowel disease. $\mathrm{Br} J$ Cancer 2009;101:1671-5.
1164 Choi CH, Rutter MD, Askari A, et al. Forty-year analysis of colonoscopic surveillance program for neoplasia in ulcerative colitis: an updated overview. Am J Gastroenterol 2015;110:1022-34.

1165 Beaugerie L, Svrcek M, Seksik P, et al. Risk of colorectal high-grade dysplasia and cancer in a prospective observational cohort of patients with inflammatory bowel disease. Gastroenterology 2013;145:166-75.

1166 Torres J, Pineton de Chambrun G, Itzkowitz S, et al. Review article: colorectal neoplasia in patients with primary sclerosing cholangitis and inflammatory bowe disease. Aliment Pharmacol Ther 2011;34:497-508.

1167 Laine L, Kaltenbach T, Barkun A, et al. SCENIC international consensus statement on surveillance and management of dysplasia in inflammatory bowel disease. Gastrointest Endosc 2015;81:489-501.

1168 Lyakhovich A, Gasche C. Systematic review: molecular chemoprevention of colorectal malignancy by mesalazine. Aliment Pharmacol Ther 2010;31:202-9.

1169 O'Connor A, Packey CD, Akbari M, et al. Mesalamine, but not sulfasalazine, reduces the risk of colorectal neoplasia in patients with inflammatory bowel disease. Inflamm Bowel Dis 2015;21:2562-9.

1170 Qiu X, Ma J, Wang K, et al. Chemopreventive effects of 5-aminosalicylic acid on inflammatory bowel disease-associated colorectal cancer and dysplasia: a systematic review with meta-analysis. Oncotarget 2017:8:1031-45.

1171 Bonovas S, Fiorino G, Lytras T, et al. Systematic review with meta-analysis: use of 5 -aminosalicylates and risk of colorectal neoplasia in patients with inflammatory bowel disease. Aliment Pharmacol Ther 2017:45:1179-92.

1172 Ungaro RC, Limketkai BN, Jensen CB, et al. Stopping 5-aminosalicylates in patients with ulcerative colitis starting biologic therapy does not increase the risk of adverse clinical outcomes: analysis of two nationwide population-based cohorts. Gut 2019;68:977-84.

1173 Grivennikov SI. Inflammation and colorectal cancer: colitis-associated neoplasia. Semin Immunopathol 2013;35:229-44.

1174 Matula S, Croog V, Itzkowitz S, et al. Chemoprevention of colorectal neoplasia in ulcerative colitis: the effect of 6-mercaptopurine. Clin Gastroenterol Hepatol 2005;3:1015-21.

1175 Jess T, Lopez A, Andersson M, et al. Thiopurines and risk of colorectal neoplasia in patients with inflammatory bowel disease: a meta-analysis. Clin Gastroenterol Hepatol 2014;12:1793-800

1176 Lu MJ, Qiu XY, Mao XQ, et al. Systematic review with meta-analysis: thiopurines decrease the risk of colorectal neoplasia in patients with inflammatory bowel disease. Aliment Pharmacol Ther 2018;47:318-31.

1177 Lashner BA. Red blood cell folate is associated with the development of dysplasia and cancer in ulcerative colitis. J Cancer Res Clin Oncol 1993;119:549-54.

1178 Lashner BA, Provencher KS, Seidner DL, et al. The effect of folic acid supplementation on the risk for cancer or dysplasia in ulcerative colitis. Gastroenterology 1997;112:29-32.

1179 Lashner BA, Heidenreich PA, Su GL, et al. Effect of folate supplementation on the incidence of dysplasia and cancer in chronic ulcerative colitis. A case-control study. Gastroenterology 1989;97:255-9.

1180 Kane SV, Acquah LA. Placental transport of immunoglobulins: a clinical review for gastroenterologists who prescribe therapeutic monoclonal antibodies to women during conception and pregnancy. Am J Gastroenterol 2009;104:228-33.

1181 Mahadevan U, Wolf DC, Dubinsky M, et al. Placental transfer of anti-tumo necrosis factor agents in pregnant patients with inflammatory bowel disease. Clin Gastroenterol Hepatol 2013;11:286-92.

1182 Zelinkova Z, van der Ent C, Bruin KF, et al. Effects of discontinuing anti-tumor necrosis factor therapy during pregnancy on the course of inflammatory bowel disease and neonatal exposure. Clin Gastroenterol Hepatol 2013;11:318-21.

1183 Bortlik M, Machkova N, Duricova D, et al. Pregnancy and newborn outcome of mothers with inflammatory bowel diseases exposed to anti-TNF- $\alpha$ therapy during pregnancy: three-center study. Scand I Gastroenterol 2013;48:951-8.

1184 Julsgaard M, Christensen LA, Gibson PR, et al. Concentrations of adalimumab and infliximab in mothers and newborns, and effects on infection. Gastroenterology 2016;151:110-9.

1185 Seow CH, Leung Y, Vande Casteele N, et al. The effects of pregnancy on the pharmacokinetics of infliximab and adalimumab in inflammatory bowel disease. Aliment Pharmacol Ther 2017;45:1329-38.

1186 Matro R, Martin CF, Wolf D, et al. Exposure concentrations of infants breastfed by women receiving biologic therapies for inflammatory bowel diseases and effects of breastfeeding on infections and development. Gastroenterology 2018;155:696-704.

1187 Bush MC, Patel S, Lapinski RH, et al. Perinatal outcomes in inflammatory bowel disease. J Matern Fetal Neonatal Med 2004;15:237-41.

1188 Morales M, Berney T, Jenny A, et al. Crohn's disease as a risk factor for the outcome of pregnancy. Hepato-Gastroenterology 2000;47:1595-8.

1189 Nielsen $\mathrm{OH}$, Andreasson B, Bondesen S, et al. Pregnancy in ulcerative colitis. Scand J Gastroenterol 1983;18:735-42. 
1190 Nielsen OH, Andreasson B, Bondesen S, et al. Pregnancy in Crohn's disease. Scand J Gastroenterol 1984;19:724-32.

1191 de Lima A, Zelinkova Z, van der Ent C, et al. Tailored anti-TNF therapy during pregnancy in patients with IBD: maternal and fetal safety. Gut 2016;65:1261-8.

1192 Kammerlander $\mathrm{H}$, Nielsen J, Kjeldsen J, et al. The effect of disease activity on birth outcomes in a nationwide cohort of women with moderate to severe inflammatory bowel disease. Inflamm Bowel Dis 2017;23:1011-8.

1193 Abdul Sultan A, West J, Ban L, et al. Adverse pregnancy outcomes among women with inflammatory bowel disease: a population-based study from England. Inflamm Bowel Dis 2016;22:1621-30.

1194 Shihab Z, Yeomans ND, De Cruz P. Anti-tumour necrosis factor $\alpha$ therapies and inflammatory bowel disease pregnancy outcomes: a meta-analysis. J Crohns Colitis 2016;10:979-88

1195 Deepak P, Stobaugh DJ. Maternal and foetal adverse events with tumour necrosis factor-alpha inhibitors in inflammatory bowel disease. Aliment Pharmacol Ther 2014:40:1035-43.

1196 Luu M, Benzenine E, Doret M, et al. Continuous anti-TNF $\alpha$ use throughout pregnancy: possible complications for the mother but not for the fetus. A retrospective cohort on the French National Health Insurance Database (EVASION). Am J Gastroenterol 2018;113:1669-77.

1197 Kanis S, Modderman S, Escher JC, et al. Long-term health outcomes of 1000 children born to mothers with inflammatory bowel disease in the anti-TNF- $\alpha$ era. United Eur Gastroenterol I 2017;5(Suppl):A149.

1198 Chaparro M, Verreth A, Lobaton T, et al. Long-term safety of in utero exposure to anti-TNF $\alpha$ drugs for the treatment of inflammatory bowel disease: results from the multicenter European TEDDY Study. Am J Gastroenterol 2018;113:396-403.

1199 Mahadevan U, Martin C, Kane SV, et al. Do infant serum levels of biologic agents at birth correlate with risk of adverse outcomes? Results from the PIANO Registry. Gastroenterology 2016;150:591-S92.

1200 Lau A, Clark M, Harrison DD, et al. Pregnancy outcomes in women exposed to the tumor necrosis factor gnhibitor, Golimumab. Ann Rheum Dis 2014;73(Suppl 2):232.2-3.

1201 Beaulieu DB, Ananthakrishnan AN, Martin C, et al. Use of biologic therapy by pregnant women with inflammatory bowel disease does not affect infant response to vaccines. Clin Gastroenterol Hepatol 2018;16:99-105.

1202 Bendaoud S, Nahon S, Gornet J-M, et al. Live-vaccines and lactation in newborn exposed in utero to anti-TNF: A multi-centre French experience in inflammatory bowel disease. J Crohn's Colitis 2018;12(Suppl 1):S527.

1203 de Lima A, Kanis SL, Escher JC, et al. Hepatitis B vaccination effective in children exposed to anti-tumour necrosis factor alpha in utero. J Crohns Colitis 2018;12:948-53.

1204 Mahadevan U, Vermeire S, Lasch K, et al. Vedolizumab exposure in pregnancy: outcomes from clinical studies in inflammatory bowel disease. Aliment Pharmacol Ther 2017;45:941-50.

1205 Julsgaard M, Kjeldsen J, Baumgart DC. Vedolizumab safety in pregnancy and newborn outcomes. Gut 2017;66:1866-7.

1206 Götestam Skorpen C, Hoeltzenbein M, Tincani A, et al. The EULAR points to consider for use of antirheumatic drugs before pregnancy, and during pregnancy and lactation. Ann Rheum Dis 2016;75:795-810.

1207 Venturin C, Nancey S, Danion P, et al. Fetal death in utero and miscarriage in a patient with Crohn's disease under therapy with ustekinumab: case-report and review of the literature. BMC Gastroenterol 2017;17:80.

1208 Mahadevan U, Dubinsky MC, Su C, et al. Outcomes of pregnancies with maternal/ paternal exposure in the Tofacitinib Safety Databases for Ulcerative Colitis. Inflamm Bowel Dis 2018;24:2494-500.

1209 van der Woude CJ, Ardizzone S, Bengtson MB, et al. The second European evidenced-based consensus on reproduction and pregnancy in inflammatory bowel disease. J Crohns Colitis 2015;9:107-24.

1210 Nguyen GC, Seow CH, Maxwell C, et al. The Toronto Consensus Statements for the Management of Inflammatory Bowel Disease in Pregnancy. Gastroenterology 2016;150:734-57

1211 Uhlig HH, Schwerd T, Koletzko S, et al. The diagnostic approach to monogenic very early onset inflammatory bowel disease. Gastroenterology 2014;147:990-1007.

1212 Malhi G, Rumman A, Thanabalan R, et al. Vaccination in inflammatory bowel disease patients: attitudes, knowledge, and uptake. J Crohns Colitis 2015;9:439-44

1213 Greveson K, Shepherd T, Mulligan JP, et al. Travel health and pretravel preparation in the patient with inflammatory bowel disease. Frontline Gastroenterol 2016;7:60-5.

1214 Rahier JF. Management of IBD patients with current immunosuppressive therapy and concurrent infections. Dig Dis 2015;33(Suppl 1):50-6.

1215 Gordon H, Steel A. Prevention of opportunistic infections in patients on biological agents for management of inflammatory bowel disease. United Eur Gastroenterol $\int$ 2014;2(Suppl):A373.
1216 Adachi JA, Ericsson CD, Jiang ZD, et al. Azithromycin found to be comparable to levofloxacin for the treatment of US travelers with acute diarrhea acquired in Mexico. Clin Infect Dis 2003;37:1165-71.

1217 National Institute for Health and Care Excellence. Quality standard [QS81] Inflammatory bowel disease. February 2015 https://www.nice.org.uk/guidance/ qs81 (Accessed 15 Dec 2018).

1218 Ricci C, Lanzarotto F, Lanzini A. The multidisciplinary team for management of inflammatory bowel diseases. Dig Liver Dis 2008;40(Suppl 2):S285-8.

1219 Garrick V, Stenhouse E, Haddock G, et al. A multidisciplinary team model of caring for patients with perianal Crohn's disease incorporating a literature review, topical therapy and personal practice. Frontline Gastroenterol 2013;4:152-60.

1220 O'Connor M, Bager P, Duncan J, et al. N-ECCO Consensus statements on the European nursing roles in caring for patients with Crohn's disease or ulcerative colitis. J Crohns Colitis 2013;7:744-64.

1221 Bennett JL, Ha CY, Efron JE, et al. Optimizing perioperative Crohn's disease management: role of coordinated medical and surgical care. World I Gastroenterol 2015;21:1182-8.

1222 Panés J, O'Connor M, Peyrin-Biroulet L, et al. Improving quality of care in inflammatory bowel disease: what changes can be made today? I Crohns Colitis 2014:8:919-26.

1223 Hibi T, Panaccione R, Katafuchi M, et al. The $5 \mathrm{C}$ concept and $5 \mathrm{~S}$ principles in inflammatory bowel disease management. J Crohns Colitis 2017:11:1302-8.

1224 Louis E, Dotan I, Ghosh S, et al. Optimising the inflammatory bowel disease unit to improve quality of care: expert recommendations. J Crohns Colitis 2015;9:685-91.

1225 Kapasi R, Glatter J, Lamb CA, et al. Consensus standards of healthcare for adults and children with inflammatory bowel disease in the United Kingdom. Frontline Gastroenterol 2019. doi: 10.1136/flgastro-2019-101260. [Epub ahead of print 19 Jul 2019].

1226 Soukop M, Robinson A, Soukop D, et al. Results of a survey of the role of multidisciplinary team coordinators for colorectal cancer in England and Wales. Colorectal Dis 2007;9:146-50.

1227 Lamb BW, Brown KF, Nagpal K, et al. Quality of care management decisions by multidisciplinary cancer teams: a systematic review. Ann Surg Oncol 2011;18:2116-25.

1228 Schraut WH. The surgical management of Crohn's disease. Gastroenterol Clin North Am 2002;31:255-63.

1229 Ke KM, Blazeby JM, Strong S, et al. Are multidisciplinary teams in secondary care cost-effective? A systematic review of the literature. Cost Eff Resour Alloc 2013;11:7.

1230 Calvet X, Panés J, Alfaro N, et al. Delphi consensus statement: Quality Indicators for inflammatory bowel disease comprehensive care units. J Crohns Colitis 2014;8:240-51.

1231 Alrubaiy L, Arnott I, Protheroe A, et al. Inflammatory bowel disease in the UK: is quality of care improving? Frontline Gastroenterol 2013;4:296-301.

1232 Lynch RW, Down C, Roughton M, et al. OC-165 No increase in surgical complication in patients treated with rescue therapy for acute severe ulcerative colitis: data from the UK IBD audit. Gut 2012;61(Suppl 2):A71.1.

1233 Lynch RW, Roughton M, Down C, et al. PWE-244 Does thromboprophylaxis in ulcerative colitis work?: data from the UK IBD audit. Gut 2012;61(Suppl 2):A397.2.

1234 Johnson MW, Lithgo K, Price T. PTH-051 The first year's outcome data from IBDSSHAMP; UK's first remote web-based self management programme for stable inflammatory bowel disease patients. Gut 2014;63(Suppl 1):A231.1.

1235 Johnson MW, Lithgo K, Price T. PTH-052 How the new inflammatory bowel disease registry and patient management system (IBD-R/PMS) has helped define the future of our district general IBD service. Gut 2014;63(Suppl 1):A231.2.

1236 Jelsness-Jørgensen LP, Bernklev T, Henriksen M, et al. Is patient reported outcome (PRO) affected by different follow-up regimens in inflammatory bowel disease (IBD)? A one year prospective, longitudinal comparison of nurse-led versus conventional follow-up. J Crohns Colitis 2012;6:887-94.

1237 Hernández-Sampelayo P, Seoane M, Oltra L, et al. Contribution of nurses to the quality of care in management of inflammatory bowel disease: a synthesis of the evidence. I Crohns Colitis 2010:4:611-22.

1238 Leach P, De Silva M, Mountifield R, et al. The effect of an inflammatory bowel disease nurse position on service delivery. J Crohns Colitis 2014;8:370-4.

1239 Coenen S, Weyts E, Jorissen C, et al. Effects of education and information on vaccination behavior in patients with inflammatory bowel disease. Inflamm Bowel Dis 2017;23:318-24.

1240 Biagini S, Giannotta M, Almerigogna V, et al. N019 The inflammatory bowel disease (IBD) dedicated day service planned by IBD specialist nurse: A good and timesaving approach to IBD or suspect IBD patients. J Crohns Colitis 2016;10(Suppl 1):S502-3.

1241 Stansfield C, Robinson A, Lal S. PTU-081 The impact of a nurse co-ordinated IBD multidisciplinary team meeting in enhancing safety and reducing cost. Gut 2015;64(Suppl 1):A96.1. 
1242 N006. Inflammatory Bowel Disease (IBD) Nurse Advice Line: a single centre experience. J Crohn's Colitis 2015;9(Suppl 1):S448.

1243 Nightingale AJ, Middleton W, Middleton SJ, et al. Evaluation of the effectiveness of a specialist nurse in the management of inflammatory bowel disease (IBD). Eur 1 Gastroenterol Hepatol 2000;12:967-73.

1244 Connell WR, Samyue T, Gibson PR, et al. Changing face of care for patients with moderate to severe inflammatory bowel disease: the role of specialist nurses in the governance of anti-TNF prescribing. Intern Med J 2015;45:1161-6.

1245 Chan W, Chen A, Tiao D, et al. Medication adherence in inflammatory bowel disease. Intest Res 2017;15:434-45.

1246 Cook PF, Emiliozzi S, El-Haji D, et al. Telephone nurse counseling for medication adherence in ulcerative colitis: a preliminary study. Patient Educ Couns 2010:81:182-6.

1247 Navarro Correal E, Benítez Leiva O, Dosal Galguera A, et al. N008 Reasons for consultation of patients with inflammatory bowel disease in telephone helplines attended by nurses. J Crohns Colitis 2016;10(Suppl 1):S498.

1248 Squires SI, Boal AJ, Naismith GD. The financial impact of a nurse-led telemedicine service for inflammatory bowel disease in a large district general hospital. Frontline Gastroenterol 2016;7:216-21.

1249 Sanromán Alvarez L, de Castro Parga ML, Hernández Ramírez V, et al. [Telematic consultations by nursing staff for patients with inflammatory bowel disease: evaluation of its capacity for resolving problems and its costs]. Enferm Clin 2014;24:102-10.

1250 Bernstein MT, Chhibba T, Walker JR, et al. A6 Preferences for care for active symptoms of ibd in a population based sample. J Can Assoc Gastroenterol 2018;1(Suppl 1):10-11.

1251 Ramos-Rivers C, Regueiro M, Vargas EJ, et al. Association between telephone activity and features of patients with inflammatory bowel disease. Clin Gastroenterol Hepatol 2014;12:986-94.

1252 Li SX, Thompson KD, Peterson T, et al. Delivering high value inflammatory bowel disease care through telemedicine visits. Inflamm Bowel Dis 2017;23:1678-81.

1253 Lloyd-Ford G, Lewis A, Hook L, et al. N008 Audit into patient opinion of an inflammatory bowel disease nurse led virtual telephone follow-up clinic. J Crohns Colitis 2014;8(Suppl 1):S357

1254 Bager P, Hentze R, Nairn C. Outpatients with inflammatory bowel disease (IBD) strongly prefer annual telephone calls from an IBD nurse instead of outpatient visits. Gastroenterol Nurs 2013;36:92-6.

1255 Fuertes F, Sathyanarayana V, Kapur K, et al. An evaluation of a pharmacist-led telephone clinic with regard to monitoring, safety and quality of service on patients taking thiopurines for the treatment of inflammatory bowel disease (IBD) and autoimmune hepatitis (AIH) at Barnsley Hospital NHS. United Eur Gastroenterol J 2015;3(Suppl):A256-7.

1256 Price T, Lithgo K, Johnson MW. PWE-062 The outcomes and benefits of moving stable inflammatory bowel disease patients from a paper based self management system to an supported, self help and management programme (IBD-SSHAMP) with specialist overview. Gut 2015;64(Suppl 1):A238.3-A239.

1257 Casey M, Hayes PS, Heaney D, et al. Implementing transnational telemedicine solutions: a connected health project in rural and remote areas of six Northern Periphery countries Series on European collaborative projects. Eur J Gen Pract 2013;19:52-8.

1258 Huang VW, Reich KM, Fedorak RN. Distance management of inflammatory bowel disease: systematic review and meta-analysis. World I Gastroenterol 2014;20:829-42.

1259 Jackson BD, Gray K, Knowles SR, et al. EHealth technologies in inflammatory bowel disease: a systematic review. J Crohns Colitis 2016;10:1103-21.

1260 Robinson A, Thompson DG, Wilkin D, et al. Guided self-management and patient-directed follow-up of ulcerative colitis: a randomised trial. Lancet 2001;358:976-81

1261 Kennedy AP, Nelson E, Reeves $\mathrm{D}$, et al. A randomised controlled trial to assess the effectiveness and cost of a patient orientated self management approach to chronic inflammatory bowel disease. Gut 2004;53:1639-45.

1262 Stansfield C, Robinson A, Lal S. OC-052 5 year follow up of a nurse led guided self management programme in ibd. Gut 2015;64(Suppl 1):A27.1-A27.

1263 Tu W, Xu G, Du S. Structure and content components of self-management interventions that improve health-related quality of life in people with inflammatory bowel disease: a systematic review, meta-analysis and metaregression. J Clin Nurs 2015;24:2695-709.

1264 de Jong MJ, van der Meulen-de Jong AE, Romberg-Camps MJ, et al. Telemedicine for management of inflammatory bowel disease (mylBDcoach): a pragmatic, multicentre, randomised controlled trial. Lancet 2017:390:959-68.
1265 National Institute for Health and Care Excellence. Ulcerative colitis: Clinical Knowledge Summaries. July 2015 https://cks.nice.org.uk/ulcerative-colitis (Accessed 15 Dec 2018).

1266 Probert C. Steroids and 5-aminosalicylic acids in moderate ulcerative colitis: addressing the dilemma. Therap Adv Gastroenterol 2013;6:33-8.

1267 Alexakis C, Oliver C, Hockney E, et al. PWE-010 Monitoring IBD medications in primary care: audit of practice in South West London. Gut 2016;65(Suppl 1):A142.1-A142.

1268 Aldulaimi D, Farmer D, Prasher $\mathrm{H}$, et al. PWE-011 Is ulcerative colitis (UC) managed adequately in primary care? Gut 2016;65(Suppl 1):A142.2-A143.

1269 Goel A, Johnson T, Limdi J. Vaccinating patients with IBD: Still to begin, at the beginning. Am J Gastroenterol 2014;109(Suppl 2):S517.

1270 Niv Y, Dickman R, Levi Z, et al. Establishing an integrated gastroenterology service between a medical center and the community. World I Gastroenterol 2015;21:2152-8.

1271 Price T, Lithgow K, Johnson MW. The UK's first internet based remote management system for monitoring stable IBD patients in the community, IBD SSHAMP (supported self help and management programme). United Eur Gastroenterol J 2013;1(Suppl):A52-3.

1272 Casellas F, Marín-Jiménez I, Borruel N, et al. Colitis ulcerosa en remisión: mejora de la adhesión terapéutica desde una perspectiva multidisciplinar. Enfermedad Inflamatoria Intestinal al Día 2016;15:37-43.

1273 Teich N, Klugmann T, Tiedemann A, et al. Vaccination coverage in immunosuppressed patients: results of a regional health services research study. Dtsch Arztebl Int 2011;108:105-11.

1274 Ruiz-Cuesta P, González-Alayón C, Jurado-García J, et al. Adherence to a predefined vaccination program in patients with inflammatory bowel disease. Gastroenterol Hepatol 2016;39:385-92.

1275 Abdallah J, Anna K, Hassan T, et al. P-049 Vaccination rates in inflammatory bowel disease. Inflamm Bowel Dis 2013;19(Suppl 1):S46.

1276 Christensen KR, Steenholdt C, Buhl SS, et al. Systematic information to healthcare professionals about vaccination guidelines improves adherence in patients with inflammatory bowel disease in anti-TNF $\alpha$ therapy. Am J Gastroenterol 2015;110:1526-32.

1277 Lomer MC, Hart AL, Verjee A, et al. What are the dietary treatment research priorities for inflammatory bowel disease? A short report based on a priority setting partnership with the James Lind Alliance. J Hum Nutr Diet 2017;30:709-13.

1278 Hart AL, Lomer M, Verjee A, et al. What are the top 10 research questions in the treatment of inflammatory bowel disease? A priority setting partnership with the James Lind Alliance. J Crohns Colitis 2017;11:204-11.

1279 Dignass A, Eliakim R, Magro F, et al. Second European evidence-based consensus on the diagnosis and management of ulcerative colitis. Part 1: definitions and diagnosis. J Crohns Colitis 2012;6:965-90.

1280 Weldring T, Smith SM. Patient-Reported Outcomes (PROS) and Patient-Reported Outcome Measures (PROMs). Health Serv Insights 2013;6:61-8.

1281 Travis SP, Farrant JM, Ricketts C, et al. Predicting outcome in severe ulcerative colitis. Gut 1996:38:905-10

1282 Ho GT, Mowat C, Goddard CJ, et al. Predicting the outcome of severe ulcerative colitis: development of a novel risk score to aid early selection of patients for second-line medical therapy or surgery. Aliment Pharmacol Ther 2004;19:1079-87.

1283 Lindgren SC, Flood LM, Kilander AF, et al. Early predictors of glucocorticosteroid treatment failure in severe and moderately severe attacks of ulcerative colitis. Eur J Gastroenterol Hepatol 1998;10:831-6.

1284 Sandborn WJ, Tremaine WJ, Batts KP, et al. Pouchitis after ileal pouch-anal anastomosis: a Pouchitis Disease Activity Index. Mayo Clin Proc 1994;69:409-15.

1285 Department of Health. The UK immunisation schedule: The Green Book, Chapter 11. March 2013 https://www.gov.uk/government/publications/immunisationschedule-the-green-book-chapter-11 (Accessed 10 Jan 2019).

1286 Goel RM, Blaker P, Mentzer A, et al. Optimizing the use of thiopurines in inflammatory bowel disease. Ther Adv Chronic Dis 2015;6:138-46.

1287 Nagle D, Pare T, Keenan E, et al. lleostomy pathway virtually eliminates readmissions for dehydration in new ostomates. Dis Colon Rectum 2012:55:1266-72.

1288 Stoffel NU, Cercamondi Cl, Brittenham G, et al. Iron absorption from oral iron supplements given on consecutive versus alternate days and as single morning doses versus twice-daily split dosing in iron-depleted women: two open-label, randomised controlled trials. Lancet Haematol 2017;4:e524-33.

1289 Gasia MF, Ghosh S, Panaccione R, et al. Targeted biopsies identify larger proportions of patients with colonic neoplasia undergoing high-definition colonoscopy, dye chromoendoscopy, or electronic virtual chromoendoscopy. Clin Gastroenterol Hepatol 2016;14:704-12. 


\section{Correction: British Society of Gastroenterology consensus guidelines on the management of inflammatory bowel disease in adults}

Lamb CA, Kennedy NA, Raine T, et al. British Society of Gastroenterology consensus guidelines on the management of inflammatory bowel disease in adults. Gut 2019;68:S1-s106. doi:10.1136/gutjnl-2019-318484.

Table 3

Table contains the original scoring where normal $=1$ (ie range 3-11) and should be normal = 0 (range 0-8). Also reference 35 in the table heading should be reference 36 .

Table 8

Legend for table 8 should be: "Endoscopic activity: Remission, score 0-2; Mild, score 3-6; Moderate, score 7-15; Severe, score $\geq 16^{\prime \prime}$

\subsubsection{UNIFI}

Results for the 8 -weekly and 12 -weekly groups for the UNIFI maintenance study are the wrong way round. The sentence "In the maintenance continuation study, 523 patients with clinical response at week eight were re-randomised to placebo, 8-weekly or 12-weekly dosing, with week 44 remission rates of $24 \%, 38.4 \%$ and $43.8 \%$, respectively" should be " "In the maintenance continuation study, 523 patients with clinical response at week eight were re-randomised to placebo, 8-weekly or 12 -weekly dosing, with week 44 remission rates of $24 \%$, $43.8 \%$ and $38.4 \%$, respectively"

\subsubsection{IBD-related cancer chemoprevention with mesalazine}

The sentence states a dose-dependent protective effect was noted with 5-ASA with a minimum dose of $1.2 \mathrm{~g} 1169$ or $2 \mathrm{~g} .{ }^{1171}$ In fact both references support a minimum dose of $1.2 \mathrm{~g}$. "or $2 \mathrm{~g}$ " should be removed from sentence.

(C) Author(s) (or their employer(s)) 2021. No commercial re-use. See rights and permissions. Published by BMJ.

Gut 2021;70:e2. doi:10.1136/gutjnl-2019-318484corr1

Check for updates 